\title{
Stochastic Effects in Quorum Sensing
}

\author{
Marc Weber
}

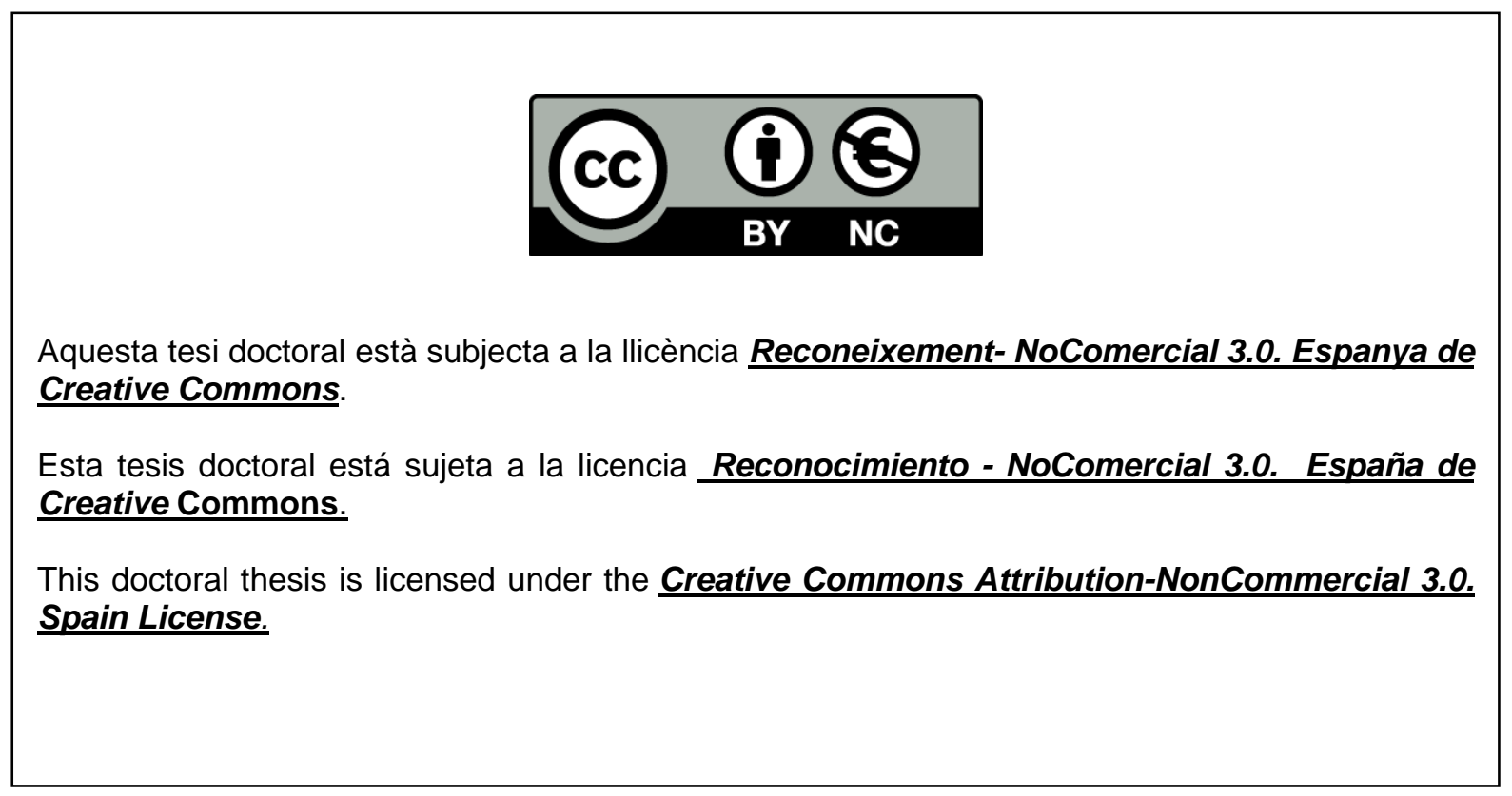


B Universitat de Barcelona

STOCHASTIC EFFECTS IN QUORUM SENSING

MARC WEBER

Ph.D. thesis

Director de tesi: Javier Buceta Fernández

Tutor: Jaume Casademunt Viader

Programa de doctorat en Física

Departament d'Estructura i Constituents de la Matèria

Universitat de Barcelona

April 2014 
Marc Weber: Stochastic Effects in Quorum Sensing, (c) April 2014 
A la Teresa, per donar-me el coratge d'estimar Als meus fills, per haver-me canviat la vida 

I M. Weber and J. Buceta. Noise regulation by quorum sensing in low mRNA copy number systems. BMC systems biology. 5:11. January 2011. Available online: link.

II M. Weber and J. Buceta. Dynamics of the quorum sensing switch: stochastic and non-stationary effects. BMC systems biology, 7:6. January 2013. Available online: link.

III M. Weber and J. Buceta. Stochastic stabilization of phenotypic states: the genetic bistable switch as a case study. PLoS ONE, 8(9):e73487, September 2013. Available online: link.

IV M. Weber and J. Buceta. book chapter "Stochasticity in Quorum Sensing". The Physics of Signaling and Communication in Bacteria. Edited by Steve Hagen. Springer. To appear.

V M. Weber and J. Buceta. Phase transition in the toggle switch coupled by quorum sensing: an Ising model in bacteria. In preparation.

VI M. Weber and J. Buceta. Colony, a stochastic simulation software for proliferating populations of cells communicating by diffusible signals. In preparation. 

Foremost, I would like to sincerely thank my Ph.D. adviser, Javier Buceta. During all these years, he has teached me a lot about how to do science, which at the end, is all about curiosity, dedication and perseverance. I am grateful for his patience, his advices, his kindness and for his availability. He has always found the time to propose improvements, discuss new ideas and push my research skills further. His close scrutiny of my academical writing has been invaluable, from our first paper published to the present text.

I would like to thank the following governmental agencies for the financial support given to me: the Spanish Ministry of Education for funding my doctoral fellowship (FPU AP2008-03272) and a research stay grant, and the Catalan government for funding part of my doctoral fellowship (2009FI Boo433).

Although the work presented in this thesis is theoretical and computational, I have been lucky to have the opportunity to immerse myself in the experimental field during a research stay at University of California in San Diego. I would like to thank all the members of the Biodynamics Lab at UCSD for their support during my stay, especially Tal Danino, Arthur Prindle and Octavio Mondragón, who answered my countless questions and taught me a lot about fluorescence microscopy, molecular biology and microfluidic devices. I am grateful to Jeff Hasty for hosting me and let me learn all the great research done in his lab. During the last years of my Ph.D. studies I was fortunate to participate to the setting up of our laboratory at the Scientific Park of Barcelona. Thanks to Rosa Baños, it was a pleasure working with you, and to Elena Rebollo for her expertise on fluorescence microscopy.

El doctorat és un viatge llarg que pot ser, de vegades, solitari. Voldria donar les gràcies a tots els meus col-legues del CosmoLab, inclouent el Javier, l'Oriol, l'Albert, el Javi, el Víctor, la Carme, el Santi, la Sogol, la Mertxe, el Roland, el Fermin, el Marc, el Iago, la 
Maria, per acompanyar-me en aquest viatge i per fer-lo més divertit. Gràcies per aquestes xerrades durant les pauses cafè, que em van permetre, entre altres coses, aprendre a parlar i a apreciar el català.

Merci à Gérard Fisher, mon professeur de physique au gymnase de Nyon, pour me transmettre sa passion pour la physique et les sciences en générale.

Finalement, je voudrais dire merci à ma famille. Sans leur support et leur aide, je ne serai jamais arrivé jusqu'où je suis aujourd'hui. Papa, maman, Àngela, Francesc, Teresa, Martí, cigronet, vous êtes tout pour moi. Teresa, gràcies pel teu suport i el teu amor. Encara que no ho sembli, hi ha molt de tu en aquesta tesi. 


\section{CONTENTS}

I INTRODUCTION I

1.1 Noise in biological systems 2

1.1.1 Origins of biochemical noise in the cell 2

1.1.2 Noise in gene expression 3

1.1.3 Intrinsic and extrinsic noise 10

1.1.4 Noise in gene regulatory networks II

1.1.5 Beneficial roles for noise 15

1.2 Quorum sensing 20

1.3 Stochastic effects in quorum sensing 30

1.4 Overview of the thesis 32

2 NOISE REGULATION BY QUORUM SENSING IN LOW MRNA COPY NUMBER SYSTEMS 37

2.1 Introduction 37

2.2 Objectives and summarized results $\quad 38$

2.3 Methods 39

2.3.1 Modeling Approach 39

2.3.2 Analytical Calculations: Null Intrinsic Noise Approximation 41

2.3.3 Parameters 47

2.3.4 Passive diffusion and active transport in QS 49

2.3.5 Validity of the low mRNA copy number approximation in the framework of protein burst expression theory 52

2.4 Results 55

2.4.1 The autoinducer dynamics exhibit different regimes as a function of the diffusion rate 55

2.4.2 The intensity of intrinsic fluctuations remains constant when changing the diffusion rate $\quad 57$

2.4.3 The total noise exhibits a maximum of intensity for intermediate diffusion rates 60 
2.5 Discussion 61

2.6 Conclusions 64

3 DYNAMICS OF THE QUORUM SENSING SWITCH: STOCHASTIC AND NON-STATIONARY EFFECTS 67

3.1 Introduction 67

3.2 Objectives and summarized results 70

3.3 Methods 71

3.3.1 Modeling of the LuxI/LuxR gene regulatory network $\quad 71$

3.3.2 Deterministic and stochastic approaches: cell growth and division 74

3.3.3 Gene expression noise: burst size $\quad 79$

3.3.4 External dilution protocol 79

3.3.5 Parameters 80

3.3.6 First passage time analysis $\quad 84$

3.4 Results 84

3.4.1 The deterministic model reproduces the experimental observations at the population level $\quad 84$

3.4.2 The stochastic simulations reveal the interplay between non-stationary effects and noise $\quad 89$

3.4.3 The features of the QS switch depends on the transcriptional noise of LuxR $\quad 97$

3.5 Discussion 101

3.6 Conclusions 106

4 STOCHASTIC STABILIZATION OF PHENOTYPiC STATES:

THE GENETIC BISTABLE SWITCH AS A CASE STUDY 109

4.1 Introduction 109

4.2 Objectives and summarized results 110

4.3 Methods 112

4.3.1 Stochastic Modification of Bifurcation Points:

Perturbative Theory 112

4.3.2 Birth and Death Process: Exact Solution 115

4.3.3 Non-null Memory Fluctuations 116

4.4 Results 118 
4.4.1 Our perturbative theory reveals the stochastic stabilization of the low state in the autoactivating bistable switch 118

4.4.2 The stochastic stabilization effect is also found in a more detailed model of autoactivating switch $\quad 124$

4.4.3 First passage time simulations reveal the interplay between intrinsic noise and stochastic modification of the energy landscape on the dynamics of the autoactivating switch $\quad 127$

4.5 Discussion 135

4.6 Conclusions 136

5 PHASE TRANSITION IN THE TOGgLE SWITCH COUPLED BY QUORUM SENSING: AN ISING MODEL IN BACTERIA 139

5.1 Introduction 139

5.2 Objectives and summarized results 142

5.3 Methods 143

5.3.1 Toggle switch 143

5.3.2 Toggle switch interfaced by quorum sensing $\quad 145$

5.4 Results 156

5.4.1 The stochastic dynamics of the cell population exhibits an unordered phase for low diffusion rate and an ordered phase for high diffusion rate 156

5.4.2 The toggle switch interfaced by QS exhibits a phase transition when varying the diffusion rate 157

5.4.3 The toggle switch interfaced by QS exhibits a phase transition during exponential growth of a colony 164

5.4.4 Cell-cell coupling enhances the population response to an external signal and leads to hypersensitive and hysteretic responses depending on the diffusion rate $\quad 169$

5.5 Discussion 173

5.6 Conclusions 179 
6 CONCLUSIONS AND PERSPECTIVES 183

6.1 Summary of original results 183

6.2 Perspectives 190

A APPENDIX 195

A.1 Dynamic cell population stochastic simulation software: Colony

195

A.2 Critical Exponents in the Ising Model 198

B SUMMARY IN CATALAN 203

B.1 Introducció 203

B.2 Resum dels principals resultats 205

BIBLIOGRAPHY 211 

From the point of view of a physicist, biological systems amaze by their tremendous complexity at different scales, ranging from the molecular level of proteins and DNA to the macroscopic scale of animals and plants. At the molecular level, cells operate far from equilibrium and are composed of complex networks of interacting genes and proteins. Their description from a theoretical point of view is challenging. However, it is now clear that physical concepts and methods are needed in order to fully understand how biological systems work. Advances in experimental methods have provided an increasing wealth of data on most components of the molecular biology of a living cell. However, how all these different components work together to give rise to biological functions remains largely unknown. Concepts from statistical physics and complex systems can be used to shed light on how these functions emerge from the interaction between genes, proteins and cells. In the molecular world of biochemical reactions, fluctuations and randomness are ubiquitous and affect many biological processes. While the effects of noise in biological systems have been widely studied, many important questions remain unanswered. At the level of a single cell, fluctuations may perturbate the functioning of gene regulatory networks. At the level of a cell population, fluctuations may interfere with cell-cell communication mechanisms and affect the reliability of multicellular coordinated behaviors, such as those observed in quorum sensing bacterial species. In this thesis, we analyze the role of noise in networks of genetic switch in bacteria, at the level of the single cell and at the level of the population. We aim at better understanding how collective behavior emerges in a population of cells communicating by quorum sensing subjected to the unavoidable influence of stochasticity. Making progress on this problem can be important for the design of synthetic circuits based on the quorum sensing com- 
munication mechanism and to gain insight into the natural function of quorum sensing in bacteria. In this thesis, we try to shed light on these questions by using mathematical modeling and stochastic simulations. In the first section of this chapter, we describe the origins and consequences of noise in biological systems. In the second section, we introduce the quorum sensing communication mechanism in prokaryotes and explain why it is a model system to study cellcell communication and multicellular behavior. In the third section, we describe how fluctuations can affect the quorum sensing communication and the experimental evidences showing that noise may play an important role in this system. Finally, in the fourth section, we briefly describe the different topics of study of the thesis.

\subsection{NOISE IN BIOLOGICAL SYSTEMS}

\subsubsection{Origins of biochemical noise in the cell}

The central dogma in molecular biology describes how genetic information flows in living cells. DNA encodes all the information necessary to produce the proteins that the cell needs to achieve its biological functions: metabolic activity, cell division, sensing and signaling, etc. The instructions stored in the DNA are read in two steps: transcription and translation. In transcription, a portion of the doublestranded DNA serves as a template and is copied into a mRNA molecule. During translation, the genetic sequence of the mRNA is used as a template to assemble the chain of amino acids that form a protein. The DNA sequence regions, called genes, that encode for proteins, are not transcribed all the time. Transcription of a particular gene can be temporarily activated or suppressed, allowing the cell to adapt to external signals and respond to a dynamically changing environment. The transcription of a gene is regulated by specific proteins called transcription factors that bind to the DNA. The whole set of genes in a cell together with their specific transcription factors form a complex regulatory network. 
All of these fundamental processes occurring in the living cell are under the unavoidable influence of noise that originates from the low number of molecules [82]. The control of transcription, for example, is mediated by the binding of a transcription factor to the upstream promoter region of a gene. Because such binding events are the result of random encounter between molecules that undergo Brownian motion, the biochemical processes regulating transcription are inherently stochastic. This molecular-level noise affects all the processes of gene expression, from the initiation of transcription to the degradation of proteins. In general, its effects are important when the numbers of molecules involved in the biochemical processes are low, as it is usually the case for mRNA, DNA and some transcription factors.

\subsubsection{Noise in gene expression}

In recent years, single-molecule in vivo experiments have demonstrated the fundamentally stochastic nature of biochemical events involved in gene expression [144]. The imaging of real-time dynamics at the level of single molecules has provided mechanistic insight into the gene expression machineries, including transcription and translation [99]. Single-molecule experiments have allowed to observe stochastic behavior that would have been otherwise obscured in an ensemble-averaged measurement. Most of these advances relate to gene expression in bacterial cells, as these are more suited for the detection of single fluorescent molecules due to their small size that limits the cellular autofluorescence background. In this context, the lac operon is one of the most studied regulatory network and a model system for understanding transcription control and stochastic gene expression in bacteria. In this thesis we will study the effects of stochasticity in genetic switches with positive feedback. The lac operon has given rise to fundamental discoveries on the stochastic activation of genetic switches and in the following we describe the details of its gene regulatory network. The transcription factor known as the lac repressor binds to its target operator site 
and represses the three downstream genes that encode proteins necessary for lactose metabolism: lac $Z$, lac $Y$, and lacA genes that encode $\beta$-galactosidase, lactose permease, and transacetylase, respectively. In the absence of lactose, LacI tetramers bind with high affinity to the lacO operator and inhibits transcription of the lac operon. LacI tetramers can also bind to other distant operator sites and form DNA loop structures, thereby enhancing the repression [98, 16]. In this condition of strong repression, very few transcripts are produced, which leads to a low level of LacY transmembrane pumps and a low lactose import rate. When lactose is present in the extra-cellular medium, it gets transported into the cell where it is hydrolized. One of the produced lactose metabolite called allolactose binds to the LacI repressor and changes its shape. The resulting complex has a lower affinity to the operator which leads to the unbinding of the repressor and the activation of lac transcription. Allolactose therefore acts as an inducer of the lac operon. Furthermore, since LacY participates in lactose import, the activation of gene expression leads to an increase in lactose transport and in the inducer concentration, which further promotes the release of the repressor. The resulting positive feedback loop has been shown to be responsible for the bistable allor-none response of the lac operon [123].

The LacI repressor is constitutively expressed at very low levels and is found at fewer than 5 copies per cell [49]. Therefore, stochasticity in the binding and unbinding of LacI to the DNA must have important effects in the regulation of the lac operon. Indeed, singlemolecule experiments have shown that the complete induction of the operon result from random and infrequent events of dissociation of the repressor from DNA [36]. While LacI has a high binding affinity to the operator sites, thermal fluctuations induce rare events of LacI full release from the binding sites. Once dissociated from DNA, it takes a timescale of minutes for the LacI tetramer to rebind to the operator, because the repressor spends most of his time binding to non-specific sequences and searching through chromosomal DNA [49]. This slow repressor rebinding time allows multiple copies of lacY transcripts to be made, resulting in a peak in the production of the transmembrane lactose permease. This sudden increase in LacY 
concentration initiates the positive feedback and switches the system to the high lac expression state. Hence, in this example, a stochastic single-molecule event as the dissociation of a repressor complex from the DNA can change a cell's phenotype. Importantly, these observations on the lac operon show that stochasticity in gene regulation can have important effects in genetic switches with positive feedback, as will be studied in chapter 3 and chapter 4 .

One of the key progress provided by single-molecule experiments has been to demonstrate that mRNAs and proteins are often produced in bursts which implies large and infrequent fluctuations in their molecular numbers [82]. Stochasticity is believed to play an important role at the two stages of gene expression: transcription and translation. At the first stage, fluctuations in the number of transcripts have been found to be larger than expected from a simple model with a continuous rate of mRNA synthesis. If the transcription process has one single limiting step, such as the RNA polymerase binding or initiation, the copy number of mRNA molecules would follow a Poissonian distribution with a variance equal to its mean. However, single-molecule studies have found that the distribution of mRNA copy number shows a significantly larger deviation than the Poisson distribution, an indication that transcription occurs in bursts. The landmark studies of Golding et al. [6o] and Le and al. [96] use the MS2-GFP method which allows one to monitor the transcription of individual mRNA in real time. The bacteria are engineered to produce the bacteriophage coat protein (MS2) fused to GFP, which has a strong affinity for a specific mRNA sequence: the ms2-binding site [101]. Following a transcription event, target mRNAs that contain repeated sequences of ms2-binding sites bind to several MS2-GFP fusion proteins. The difference in the diffusion coefficient of free MS2-GFP protein and mRNA-MS2-GFP complex can then be used in order to detect single mRNA molecules in real time by analyzing the fluorescence signal, a method called fluorescence correlation spectroscopy $[148,96]$. The results of these studies showed transcriptional bursting by directly counting the integervalued numbers of mRNA in living E. coli cells. The observed behavior can be easily explained by a simple model $[132,88]$ of gene 
activation-inactivation in which the gene in the off state switches to the on state with constant probability, and the gene in the on state either switches off or makes a transcript with constant probability (see figure 1.1A-D). Transitions between the two states of the gene are assumed to happen at time intervals that are exponentially distributed (constant probability of transition), following a random telegraph process [73]. During each "on" period, transcription is assumed to be Poissonian and produces a random number of mRNA molecules. The combined effect of the exponential duration of active periods and the Poissonian transcription is that the number of mRNAs produced during each active period follows a geometric distribution [20]. In this model, if long periods of inactivity are followed by short periods of intense mRNA synthesis activity, transcription is said to occur in bursts (see figure 1.ID). One of the consequences of these findings is that fluctuations in the level of mRNA are expected to be particularly large when the transitions between the two states of the gene are slow compared to the mRNA lifetime. Although this seems to be the case for the lac operon, where binding and unbinding rates of the LacI repressor have been measured, transcriptional bursting have also been observed for constitutive promoters [99], where there is no known transcription factor binding and unbinding. Other mechanisms may be responsible for this behavior, such as transcription re-initiation due to retention of sigma factor during the elongation process [43] or mRNA polymerase availability. In eukaryotes, other specific processes are believed to be responsible for transcriptional bursting, such as the slow kinetics of chromatin remodeling [158].

Stochasticity also arises at the second stage of gene expression, when mRNA molecules are translated into functional proteins (see figure 1.1E-G). Fluctuations in the number of transcripts are transmitted to the concentration of proteins and can be either amplified or reduced depending on the details of the gene expression mechanism. Under repressed conditions, single-molecule experiments have shown that proteins are also produced in bursts $[202,30]$. In E. coli, individual molecules of yellow fluorescent protein (YFP) fused to a membrane protein could be monitored in real-time [202]. The reduced 
diffusion of the membrane-bound protein allowed to increase the sensitivity of the fluorescence signal detection and to identify individual proteins. The proteins were found to be produced in bursts resulting from the transcription of a single mRNA molecule from the strongly repressed lac operon. The statistics of protein production were found to be well described by a simple model of uncorrelated bursts parameterized by two values, the average number of proteins produced per burst, $b=4.2$, and the average number of bursts per cell cycle, $a=1.2$. Moreover, the number of proteins produced in each burst followed a geometric distribution, which can be interpreted by a simple biological model. During the lifetime of a mRNA, several rounds of translation take place. After the initiation of one translation round, the presence of the ribosomes confers protection against ribonucleases and prevents the degradation of the mRNA. After the completion of translation, the ribosome unbinds from the mRNA and ribonucleases compete with other ribosomes for binding to the mRNA. It was shown theoretically that the probability of producing $n$ proteins from one mRNA molecule follows the geometric distribution $[20,111,51] P(n)=\rho^{n}(1-\rho)$, where $\rho$ is the probability of ribosome binding and $1-\rho$ is the probability of ribonuclease binding. Moreover, assuming that the bursts are uncorrelated and exponentially distributed (following a Poisson process) and that the mRNA lifetime is much smaller than the protein lifetime (which is usually the case in prokaryotes [178]), the resulting steady-state distribution of proteins is the gamma distribution [30]

$$
p(x)=\frac{x^{a-1} e^{-x / b}}{b^{a} \Gamma(a)} .
$$

where $\Gamma$ is the gamma function and $x$ is the continuous approximation of the protein copy number $n$. The gamma distribution derived from this translational burst model compares well with stochastic simulations of gene expression models when transcription occurs at a constant rate (see figure 1.IE-G). 
Figure 1.1 (following page): Stochastic model of gene expression. (A) The threestage model of gene expression describes the basic biochemical reactions for the expression of a single gene controlled by a repressor (adapted from [82]). The promoter can transition between the active form $(A)$ and the repressed form $(R)$ with transition rates $k_{\text {off }}$ and $k_{\text {on }}$. Transcription and translation are modeled as first-order reactions with rates $s_{A}$ (transcription rate when promoter is active), $s_{R}$ (transcription rate when promoter is repressed) and $s_{P}$ (translation rate), as well as degradation of mRNA and proteins, $\delta_{M}$ and $\delta_{P}$. In a stochastic model of gene expression, each reaction step is described as a random event with an exponentially distributed reaction time. The randomness in the reactions is expected to become more important as the number of molecules involved is low. Deterministic models, on the other hand, cannot capture the effects of stochasticity in gene expression. (B-D) Time series and histograms of mRNA concentration from stochastic simulations using the Gillespie algorithm [57] in the transcriptional burst model. Fixed parameters are $s_{R}=0.5, \delta_{M}=0.1$ and cell volume $V=1.5 \mu \mathrm{m}^{3}$. All reaction rate units are $\min ^{-1}$. (B) Fast promoter transitions compared to the mRNA lifetime lead to small fluctuations in the number of mRNA molecules. $k_{\text {off }}=k_{\text {on }}=5, s_{A}=10$. (C) Slow promoter transitions lead to large mRNA level fluctuations and bimodal distribution. $k_{\text {off }}=k_{\text {on }}=0.02, s_{A}=10$. The average number of mRNA is the same as in (B). (D) When the stability of the repressed promoter is increased and becomes active only for short and infrequent periods of time, the transcription occurs in bursts. $k_{\text {off }}=1, k_{\text {on }}=0.02, s_{A}=250$. The average number of mRNA is the same as in (B). (E-G) Time series and histograms of protein concentration from stochastic simulations using the Gillespie algorithm [57] in the translational burst model. The promoter is assumed to remain in the active state (constitutive expression). Fixed parameters are $s_{R}=0.5, \delta_{M}=0.1, \delta_{P}=0.05$. (E) High transcription rate and low translation rate produce small fluctuations in protein number. $s_{A}=5, s_{P}=0.1$. (F) Lowering transcription rate and increasing translation rate lead to an increase in protein fluctuations. $s_{A}=0.2, s_{P}=2.5$. (G) Lowering transcription rate and increasing translation rate further produce bursts of protein production. $s_{A}=0.02, s_{P}=25$. (E-G) In these three cases the average number of proteins is the same. The histograms from stochastic simulations match well with the gamma distribution derived from a simple translational burst model (red line) with burst frequency parameter $a=s_{A} / \delta_{P}$ and burst size parameter $b=s_{P} / \delta_{M}$. 

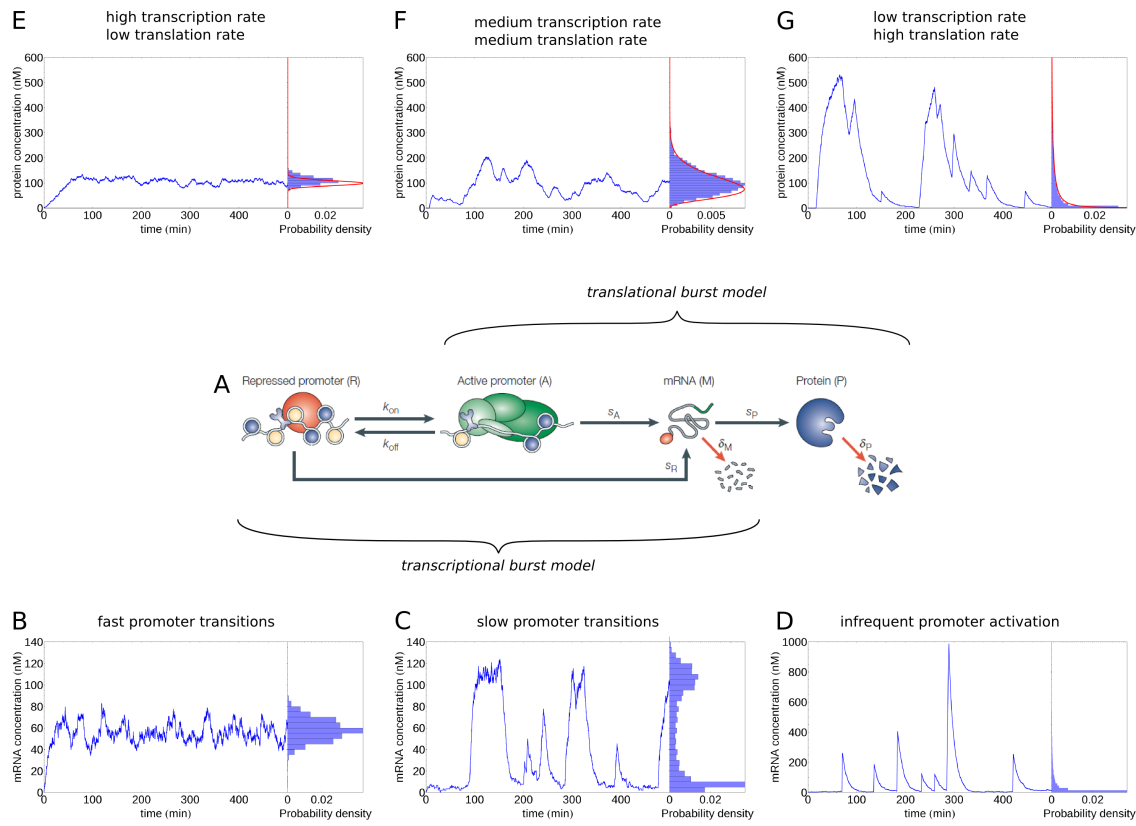
While single-molecule experiments have demonstrated the stochastic nature of gene expression, their results were specific to the promoters and the details of gene regulation mechanisms. How gene expression noise depends on these details is still an open question [99] and other mechanisms could also influence the overall noise in this process. For example, supercoiling of the chromosome in cyanobacteria may play a role in the stochasticity of its circadian rhythm [34]. The contributions of transcriptional and translational bursting to the total noise in gene expression depend on the details of the gene regulation. However, it is generally assumed that translational bursting is the main source of noise in prokaryotes [82]. In the landmark study of Ozbudak et al. [124], both noise sources were tuned by changing independently the rates of transcription and translation of a GFP gene while maintaining the same average concentration of proteins. A low rate of transcription combined with a high rate of translation produced large fluctuations in the protein concentration, showing that translational bursting is the main source of stochasticity, in agreement with the simple gene expression model (see figure 1.1E$\mathrm{G)}$.

Theoretical efforts have been done in order to model both the transcriptional and translational stochasticity. In the three-stage model of gene expression of Shahrezaei et al. [166], the transitions between inactive and active promoter states, the transcription process and translation process are all treated stochastically. The distribution of protein numbers could be calculated using some approximations that are valid when the mRNA lifetime is much shorter than the protein lifetime. Under these conditions, the calculations showed that the more realistic three-stage model reproduced the geometric distribution for protein synthesis and therefore validated the simpler translational burst theory.

\subsubsection{Intrinsic and extrinsic noise}

While the inherent randomness of biochemical reactions is at the origin of molecular noise, external sources of fluctuations can also 
affect the cellular processes. For example, fluctuations in the number of ribosomes, in the activity level of mRNA polymerase, in the temperature, in the growth rate or in the concentration of external signal in the environment can all modulate the production and degradation rates of a given protein and modify the fluctuations in its concentration. Such sources of noise are usually called extrinsic (or external) noise, while the molecular noise is referred to as intrinsic noise. The differentiation between these two categories of noise sources in the case of gene regulation was formally introduced in the theoretical work of Swain et al. [177] and in the experiments of Elowitz and al. [50]. In the latter experiments (see figure 1.2), E. coli cells are engineered to simultaneously express cyan and yellow fluorescent proteins that are under the control of two identical promoters. In this setup, extrinsic fluctuations are those that affect equally the expression of both fluorescent proteins. For example, fluctuations in the number of available ribosomes should modulate equally the translation rate for both proteins. Intrinsic fluctuations, on the other hand, are those inherent to the randomness of transcription and translation, and affect independently the two gene expression pathways. The contribution of intrinsic noise to the total fluctuations can therefore be related to the uncorrelated part of the fluctuations in the expression levels of the two proteins. The results showed that both noise sources contribute to the total variability in the cell and that the relative importance of intrinsic to extrinsic noise depends on the promoter. Moreover, the time scale for intrinsic fluctuations in bacteria is smaller than 9 minutes and can be considered as white noise within the limit of the experimental precision, whereas the extrinsic fluctuations act on a time scale similar to the cell cycle duration [153].

\subsubsection{Noise in gene regulatory networks}

At the level of a single gene, fluctuations clearly affect the gene expression process and result in variability in the number of protein molecules present in the cell. A natural question arising from this 


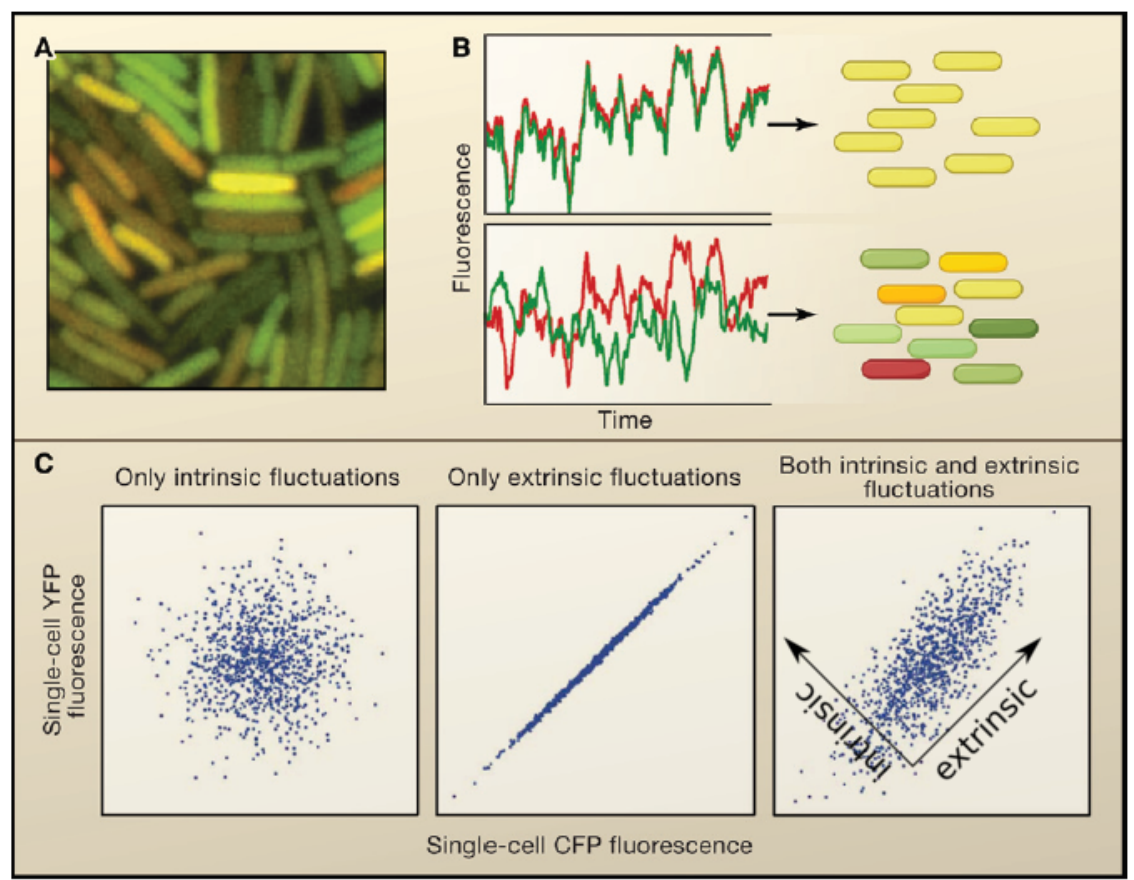

Figure 1.2: Intrinsic and extrinsic noise in gene expression. Engineered strains of E. coli [50] express a cyan fluorescent protein gene (cfp, shown in green) and a yellow fluorescent protein gene (YFP, shown in red) controlled by identical promoters. (A) Fluorescent image of cell colony with combined CFP and YFP channels. Cells with the same amount of YFP and CFP appears in yellow, while cells with different amounts of proteins appear either in red or in green. (B) Schematic representation of the fluorescence time course in a single cell. In the absence of intrinsic noise (upper) the protein levels fluctuate in a correlated manner. Intrinsic noise may introduce uncorrelated fluctuations leading to differences between YFP and CFP protein levels (lower). (C) Scattered plot of fluorescence in three scenarios: intrinsic noise only, extrinsic noise only, or both. The intensity of extrinsic noise is proportional to the spreading in the direction of the diagonal and intrinsic noise intensity to the spreading in the direction perpendicular to the diagonal. Adapted from [143] and [50]. 
observation is how fluctuations are handled within gene regulatory networks, which control most cellular functions. How does noise influence the ability of regulatory networks to transmit reliable information, such as a response to an external signal? All sources of noise are likely to affect in some way the regulation at different levels of the network organization, from simple gene auto-regulation to genome-wide regulatory networks.

One of the simplest case of gene regulation is the self-inhibited gene, where the gene encodes for a transcription factor that binds to its own promoter region and represses its expression. Several studies have shown that such a negative auto-regulation mechanism reduces the variability in the protein level $[17,9,46]$. In the experiment of Becskei and Serrano [17], a single gene negative feedback system was engineered in E. coli cells. They compared the variability in the protein expression level for this regulatory network with that obtained in the absence of feedback control. They observed a decrease in the variability in the network with negative feedback, as predicted by mathematical modeling [128, 182, 169, 129]. Such as noise reduction effect can be expected to play an important role in many gene regulatory networks. In fact, negative auto-regulation is widespread in prokaryotic regulatory networks and roughly $40 \%$ of known transcription factors in E. coli repress themselves [167]. Negative feedback may also play a role in minimizing the noise on downstream processes, by shifting the noise to higher frequencies where it is more easily filtered out by gene networks as demonstrated theoretically [169] and experimentally [9].

The other type of simple auto-regulation consists of a gene encoding for a transcription factor that activates its own transcription. Self-activating feedback loop can lead to bistability, as shown by mathematical modeling [76], a behavior that has been observed in a number of biological systems [1, 175], as in the case of the lactose utilization network in E. coli [123], and has also been implemented in synthetic circuits $[56,18,76,8,10]$. The cooperativity in the gene regulation by transcription factors together with the self-activation result in a switch-like behavior where the stationary expression of the gene can have two distinct states: one with low expression level, the 
"off" state, and the other with high expression level, the "on" state. Due to the cooperativity, a minimum amount of transcription factor is needed in order to trigger the activation. When in the "off" state, transcription factor level stays below the threshold concentration indefinitely and only large fluctuations can induce the gene expression and switch the cell to the "on" state $[68,52]$. Similarly, fluctuations may also switch a cell to the "off" state. The transitions between the stable states occur due to random fluctuations and are inherent to the details of the genetic network. However, additional feedbacks may be used to control the transition rates of the bistable system. Avendaño et al. [10] constructed an interlinked positive and negative feedback network based on the galactose uptake control system of Saccharomyces cerevisiae and showed that tuning the strength of the coupled negative feedback allowed to tune the frequency of the transitions. These results highlighted the role of the noise modulation by the two different feedback loops. Indeed, positive and negative feedbacks modulate differently the noise [143], which result in a complex noise regulation when both feedback loops are combined. At the level of the cell population, stochastic transitions in a bistable system lead to a bimodal population distribution. By differentiating cells into two distinct stable states, positive feedback regulation enhances population heterogeneity and provides a mechanism for creating phenotypic variability in a population of genetically identical cells. Positive feedback loops therefore allow for a simple mechanism of cell differentiation and cell decision making and has been the subject of intensive theoretical study [143]. While the basis of stochastic transitions in the positive feedback switch has been extensively studied, open questions remain about the effects of stochasticity in such a system (see chapter 4 ).

Negative and positive autoregulation are not the only regulatory motifs that appear in transcriptomes. For example, direct interaction between a transcription factor and a target gene represents one of the simplest building block of regulatory networks. Fluctuations in the concentration of the transcription factor can be transmitted to the target gene, a phenomenon known as noise propagation. In the study of Pedraza and Van Oudenaarden [134], a synthetic reg- 
ulatory cascade of three genes was built such that the first gene represses the second and the second gene represses the third. By measuring the expression level of the three genes using multiple fluorescent reporters, the authors found that fluctuations were transmitted from the upstream genes to the downstream genes. As theoretically shown $[134,29]$, the total variability in the expression of one gene can be decomposed into three components: the intrinsic noise of the specific gene, transmitted noise from the upstream gene and extrinsic noise coming from global fluctuations. The transmitted noise sources are modulated by the strength and type of interactions between genes. Thus, even in a network where all the components have low intrinsic noise, the transmitted noise can still be substantial and stochasticity may be important. Other important regulatory motifs include feed forward loops, which modulate noise differently depending on the type of interaction (positive or negative) between the transcription factor and the target genes $[167,35]$.

\subsubsection{Beneficial roles for noise}

As a perturbation to the reliable functioning of cellular functions, noise is a nuisance. This is especially true for cellular processes whose success relies on precise spatial and temporal transmission of information, such as in the development of multicellular organisms. Evidences of the existence of noise buffering mechanisms have been found in the development pathways of Drosophila [172] and Caenorhabditis elegans [145] and suggest that these organisms have evolved mechanisms to minimize noise during development. However, noise may also have beneficial roles in many biological processes. In the recent years, an increasing number of studies have shown that noise provides useful physiological functions in single cells and produces variability in cell populations that can be advantageous [48]. At the level of intracellular functions, noise has been suggested to improve regulation by coordinating the expression of large groups of genes. Such a case of stochastic enhancement of gene expression coordination has been studied in the experimental work 
of Cai and al. [31], which examines the yeast response to extracellular calcium. The main transcription factor regulating the response to calcium is Crzi, whose activity is controlled by phosphorylation and dephosphorylation. The presence of calcium favors the dephosphorylation of Crz1, which results in its localization to the nucleus. Surprisingly, experiments showed that Crzi localizes to the nucleus in short stochastic bursts lasting 1-2 minutes and that calcium concentration modulates the frequency of these bursts without changing their average duration and amplitude. Such a frequency-modulation regulation allows cells to co-regulate a large set of target genes with different input functions while maintaining the same proportionality with respect to the signal concentration [48].

At the level of cell population, noise provides a fundamental function by allowing phenotypic variability in a population of genetically identical cells. This variability could be especially useful for unicellular organisms, by enabling population-level strategies such as bethedging and division of labor that would be difficult to implement in a deterministic system [48]. Population variability is usually enhanced by regulatory networks that produce multiple gene expression profiles that are mutually exclusive and that correspond to a particular cellular decision. These states are stable in the sense that small variations in gene expression or fluctuations in the environmental signals are insufficient to induce a transition from one state to another and the cell can remain in the same state for durations superior to the cell cycle, providing a mechanism of epigenetic inheritance. Occasionally, large fluctuations can drive the cell to a different stable state, the mechanism creating diversity in the population. As described above, positive feedback is a simple regulation motif that creates bistability, allowing for the "on-off" switching of a particular genetic program. The lactose utilization network of E. coli [123] is the canonical example of this type of behavior, where cells stochastically transition between the lactose and the glucose metabolism. In general, cells can have several stable states as a result of the complexity of gene regulatory networks, leading to multistability.

These observations raise the fundamental question of how phenotypic variability can be advantageous for a cell population and 
under which conditions. One of the key ideas emerging from theoretical studies $[183,92]$ is that stochastic phenotypic switching could provide a fitness advantage in a fluctuating environment. When the concentration of nutrients fluctuates randomly, cells can basically choose between two strategies to optimize their growth: sensing the changes and responding appropriately by switching their phenotype (changing metabolic pathway) or allowing a fraction of the population to stochastically differentiate to a phenotype that is adapted to expected future changes in food concentrations, thus anticipating environment fluctuations. The cost of the former strategy is maintaining active sensory machinery and usually slow response times, whereas the second strategy essentially sacrifices the part of the population that has a suboptimal growth in the current environment. The advantage of the sensing strategy is that all the cells may achieve an optimal growth after the transient time of response to an environmental change, whereas the stochastic switching strategy does not need to maintain any sensory network and can allow a group of cell to be well-adapted to any abrupt environment change. The latter advantage is especially true in the case of a sudden and severe stress, where the existence of a group of resistant cells can optimize the survival of the population [23]. The experimental study of Acar and al. [2] showed that the phenotypic switching rate may influence the growth advantage of a population of yeast cells exposed to a fluctuating environment. Fast switching cells grow faster in a rapidly fluctuating environment whereas slow switching cells present a growth advantage when the environment changes more slowly. The stochastic switching is therefore an alternative strategy to sensing and is most effective when the rate of phenotypic switching is similar to the rate at which the environment fluctuates.

Another example of beneficial variability produced by noise is the competence regulatory network in Bacillus subtilis. Competence is a stress response activated in conditions of nutrient limitation that allows cells to take up DNA from the environment. The differentiation into the competent state is transient and occur only in a small fraction of the cells, while the rest of the population remain in the vegetative state or commit irreversibly to sporulation. At the heart 

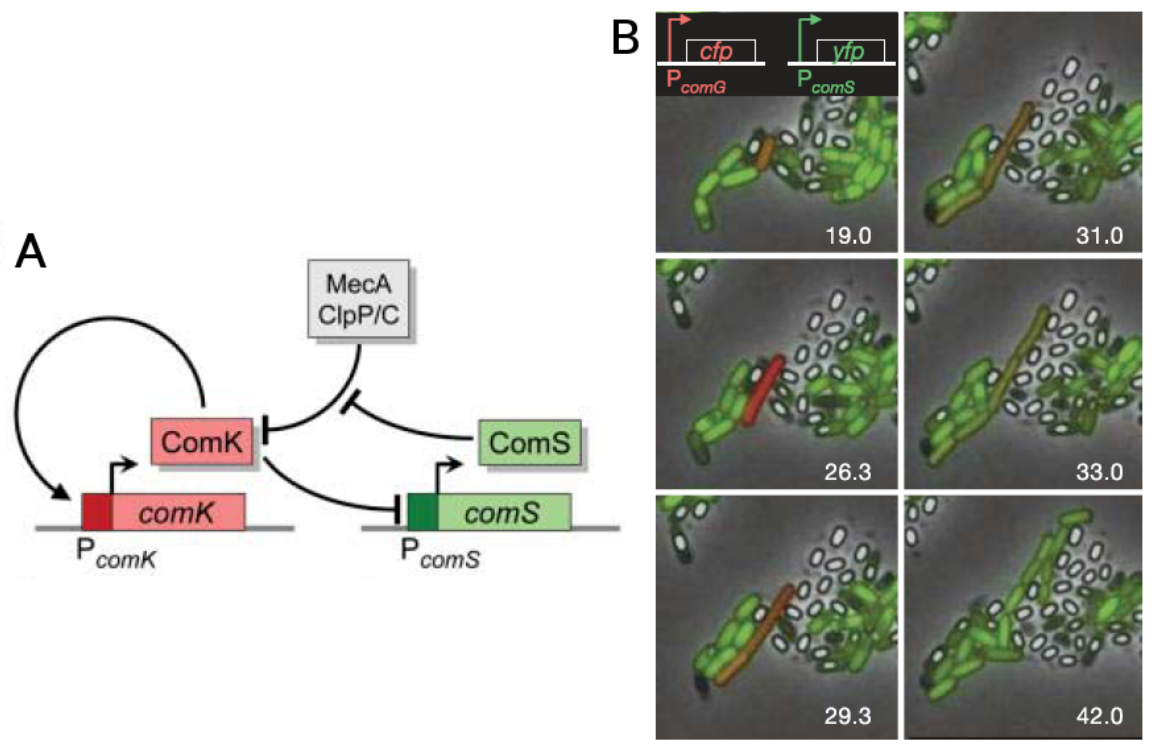

Figure 1.3: Probabilistic differentiation into the competence state in Bacillus subtilis. (A) The core circuit controlling the competence state includes the positive feedback self-regulation of ComK, which leads to bistability. The competence genes are activated when the cell is in the high ComK state. In the vegetative state, ComK levels are kept low by the MecA-ClpC-ClpP protease complex which degrades ComK. ComS competes with ComK for binding to the protease complex, effectively lowering the rate of ComK degradation. Random fluctuations may drive ComK level above a threshold and induce transition to the competent state. An indirect negative feedback loop mediated by ComS reduces ComK level and induces the exit from competent state. Adapted from [176]. (B) Fluorescence images from a typical competence event with $\mathrm{P}_{c o m s}-y p f$ and $\mathrm{P}_{c o m G}-c f p$ (a reporter of $\mathrm{P}_{\text {comK }}$ activity) expression shown in green and red, respectively. The cell exhibits a pulse of comK expression (red), during which the competence is active. Adapted from [175]. 
of the competence control circuit are the regulatory proteins ComK and ComS (see figure 1.3). ComK positively regulates its own expression producing a bimodal population distribution where competent cells exhibit high levels of ComK, activating the downstream genes involved in DNA uptake, and noncompetent cells produce low basal amounts of ComK [175]. Additional negative feedback loops mediated by ComS and the ClpP-ClpC-MecA protease complex leads to a system combining positive and negative feedback loops that exhibits excitability, a type of behavior similar to the action potential in neurons. In this system, intrinsic noise is thought to play an essential role in the initiation of competence by allowing ComK to surpasses the threshold level necessary for the activation of the positive feedback. This possibility was tested in the experimental work of Süel and al. [176] by reducing the total intrinsic noise using very large cells that are unable to divide, and in the experimental study of Maamar and al. [110] by increasing the comK transcription rate and lowering the translation rate, thereby reducing the noise in gene expression. Moreover, intrinsic fluctuations may be also responsible for the variability in the durations of competence events.

As we have seen, noise plays an important role in many biological functions and is ubiquitous in cellular processes, such as in transcription, translation and gene regulation. Its effects are especially important in cell differentiation circuits, such as genetic switches. By allowing transitions between the different stable states, noise enhances the phenotypic variability. How biochemical noise drives these transitions, modifies the dynamics of the gene circuit and affect the phenotypic landscape is still an open question. In chapter 4, we study this problem and show that noise can redesign the phenotypic landscape of a positive feedback genetic switch.

If noise impacts all of the intracellular processes, the same could be true for the processes of information exchange with its surroundings. Cells, in their natural context, are often surrounded by other cells, sense and respond to changes in their environment and communicate one with another. This characteristic may be obvious in multicellular organisms, but is also true for simpler prokaryotic cells, as will be seen in the next section. 


\subsection{QUORUM SENSING}

Bacteria, long thought having a solitary existence, were found to communicate with one another by sending and receiving chemical messages [13]. With the discovery of what is now called quorum sensing (QS), it became clear that bacteria possess complex communication systems that allow them to coordinate their behavior in a way similar to multicellular organisms. By sending and receiving chemical messages, bacteria are able to gather information about their environment and control gene expression synchronously in the whole population. This ability to generate a collective behavior in response to environmental changes resembles the sophisticated communication mechanisms found in eukaryotes, such as the community effect during development [157]. In the simplest form of quorum sensing, cells produce, export into the environment and detect small chemical-signaling molecules called autoinducers. At low cell density, the autoinducer molecules are diluted into the medium and are present at a low concentration. As the population of bacteria grows, more cells produce and release the autoinducer and its concentration increases. Upon reaching a critical concentration of signaling molecule, bacteria are able to detect its presence and initiate a signaling cascade resulting in changes in the expression of target genes. By exchanging the autoinducer molecules through their environment, bacteria are therefore able to sense their own number and adapt their behavior depending on the average cell density of the colony. Furthermore, communication by QS allows bacteria to coordinate gene expression in the entire population, providing a simple mechanism for achieving collective behavior.

The concept of prokaryotic intercellular communication originates with the discoveries of Tomasz [186] on genetic competence in Streptococcus pneumoniae and of Nealson, Platt and Hastings [119] on the bioluminescence in Vibrio. Nealson and al. showed that the small marine bacterium Vibrio fischeri produces light at high cell density but remains obscure in diluted suspension. Light production could be stimulated by the addition of cell-free culture fluids, suggesting the existence of a hormone-like substance secreted by the cells, called 
the autoinducer, which was later identified as an acyl-homoserine lactone (AHL) [47]. During the exponential growth of a freshly inoculated culture of bacteria, the light emission was found to increase abruptly upon reaching a critical cell density. It is now known that such "all-or-nothing" behavior is caused by the positive feedback loop mediated by the LuxI protein responsible for the autoinducer synthesis (see page 26) [198, 121]. The QS mechanism in $V$. fischeri allows bacteria to regulate the production of light depending on the cell density, which may provide them with a great adaptability to survive in different environments. When examining the wild life of the bacterium, one finds that it leads two very different lives: either free-living and isolated in seawater or forming a dense colony in the light organ of the Hawaiian bobtail squid Euprymna scolopes (see figure 1.4). The association between the bioluminescent bacterium and the squid is a fascinating example of symbiosis [122, 155, 14], in which the animal host provides nutrients to the bacteria, and the bacteria provide light to the host, allowing it to escape its predators more easily. Euprymna scolopes is a small bobtail squid which lives in shallow coastal waters off the Hawaiian Islands and Midway Island [155]. During its nocturnal foraging activity, the squid uses the light emitted by $V$. fischeri from its ventral surface to camouflage itself in a behavior called counter-illumination. By adjusting the intensity of the bioluminescence from its light organ to match down-welling moonlight, E. scolopes is able to reduce its silhouette and evade predators more easily [80]. The light organ (see figure 1.4) hosting the bioluminescent bacteria is a complex organ capable of modulating the intensity of light and redirect it in the ventral direction using a reflective tissue layer [40]. In the squid light organ, $V$. fischeri grows to extremely high cell densities (approximately $10^{11}$ cells $/ \mathrm{ml}$ ) [154] where it produces and accumulates autoinducer molecules. The high concentration of autoinducer activates the signaling cascade that results in the induction of the expression of the genes required for light production. On the other hand, when $V$. fischeri lives in open seawater, the autoinducer it produces is quickly diluted and the light production genes are turned off. The bacterium therefore uses the QS mechanism to detect wether it lives in a symbiotic associ- 
ation with its host or in the open seawater. When fully induced, light production consumes a considerable part of the metabolic energy of the cells and can account for up to $20 \%$ of oxygen consumption [26]; the concentration of luciferase, the key enzyme responsible for light emission, can constitute $5 \%$ or more of the total cellular proteins [67]. In the seawater environment, $V$. fischeri cells therefore avoid expending the energy for light emission which results in a fitness advantage. In this case, the QS mechanism enables bacterial cells to adapt to very different ecological niches. Interestingly, the squid Euprymna scolopes follows a curious diel behavior [122]: each day at dawn, when it buries into the sand for their daytime quiescence, the squid expels $95 \%$ of the light-organ symbionts into the seawater. During the day, the bacteria that remain in the host grow till reaching the maximum population size. When the squid emerges at dusk to hunt, its light organ is full of bioluminescent bacteria which provide him with camouflage light. This behavior is believed to not only help maintain the symbiont population in the host but also to seed the environment with bacteria, increasing the chances of establishing symbiosis with uninfected juvenile squids. It also indicates that $V$. fischeri cells are faced with frequent changes in their environment and that QS may offer an advantageous strategy to enhance their adaptability.

Over the past two decades, a growing number of bacterial species have been found to use quorum sensing mechanisms to control a variety of cellular functions [121, 14]. Vibrio harveyi, a free living marine bacterium and an important pathogen of marine organisms, produces and responds to three different autoinducers [121], forming a complex QS system that controls the bioluminescence pathway. In Pseudomonas aeruginosa, an opportunistic human pathogen that primarily infects individuals who are immunocompromised, several QS pathways have been found to regulate biofilm development and virulence factor production [130, 42]. In Vibrio cholerae, the causative agent of the human disease cholera, pathogenicity and biofilm formation are controlled by two parallel QS systems [206, 70, 81]. Interestingly, at low cell density $V$. cholerae activates the expression of virulence factors and forms biofilms, whereas the accumulation of 

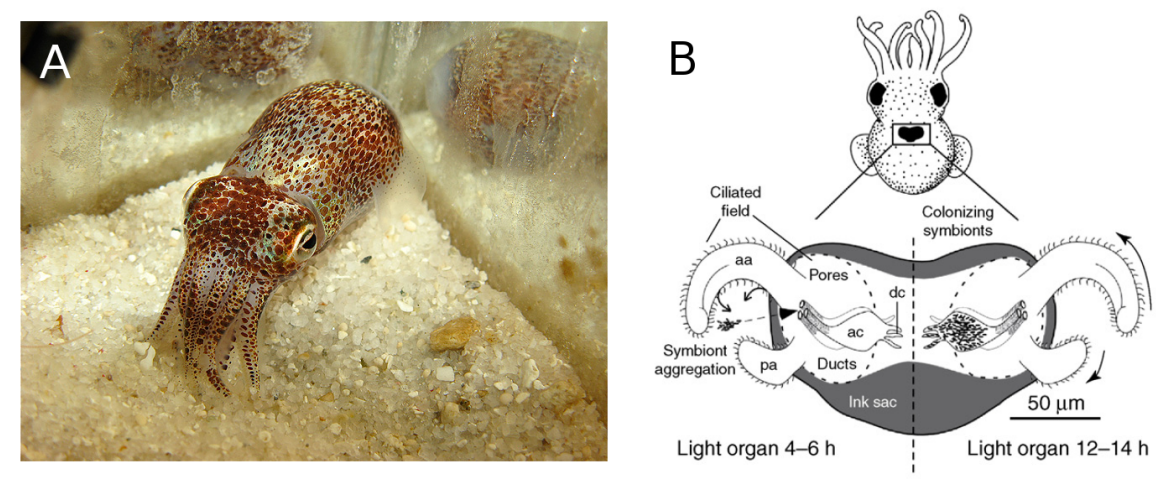

Figure 1.4: Light organ of the bobtail squid Euprymna scolopes hosting the bioluminescent bacteria Vibrio fischeri. (A) Photography of the Hawaiian bobtail squid Euprymna scolopes, which measures about 40 $\mathrm{mm}$ at adult age. Photography by Spencer Nyholm. (B) Cutaway illustration of the juvenile light organ of Euprymna scolopes, early (left) and late (right) in the initiation of symbiosis. When symbiosis is established, Vibrio fischeri bacteria live in the deep crypts (dc) of the light organ. In this association, the host uses light that is produced by the bacteria in counter-illumination to avoid predators during their nocturnal behavior, while the bacteria receive nutrients from the host. Adapted from [193]. 
autoinducers at high cell density represses these traits and promotes the dispersion of bacteria, allowing them to leave the host and initiate a new cycle of infection. This behavior is the opposite of what is seen in many other pathogens, which activate virulence factor expression at high cell density. In Streptococcus pneumoniae and Bacillus subtilis, QS controls the activation of competence, the ability to uptake exogenous DNA. Cell-cell communication by QS is also found in soil bacteria species, as for example Agrobacterium tumefaciens, a plant pathogen that causes the crown gall disease.

In natural environments, bacteria are often found in mixed-species communities where cells exchange chemical signals across species $[13,174,14]$. This new level of complexity in bacterial collective behavior gives rise to the concept of socio-microbiology [173, 161] which studies the cooperative or cheating behaviors in a microbial population. Some interesting questions in this field include how QS can be an evolutionarily stable strategy and what is its role under ecological relevant conditions. One category of polymicrobial interaction of special interest is the association of pathogenic bacterial species [137]. Pseudomonas aeruginosa, for example, is a highly adaptable bacterium which inhabits very diverse environments and is also known as an opportunistic pathogen, causing infections of the eyes, ear, skin, urethra and respiratory tract in cystic fibrosis and burn patients [180]. Three QS systems allow the bacterium to regulate the synthesis of several virulence factors and communicate with other microbes, enabling sophisticated functions to interact with other bacteria. $P$. aeruginosa secretes antimicrobial chemicals to compete with other bacteria but also uses QS signals to cooperate with other species $[156,180]$.

As the most abundant form of life on earth, bacteria are also often found to live in association with other organisms. Inter-kingdom communication systems have evolved that allow bacteria to interact with eukaryotic organisms [174], increasing the complexity of behaviors such as parasitic, symbiotic or commensal relationships. One interesting example is the establishment of symbiosis between bacteria of the family Rhizobiaceae and leguminous plants. Successful symbiosis leads to the formation of root nodules that host populations 
of Rhizobiaceae bacteria that mediate $\mathrm{N}_{2}$ fixation. In the formation of the nodules, complex two-way chemical communication between the two species are involved, including QS signaling between bacteria, flavonoids compounds release by the roots which signal the bacteria to assemble near the root surface and production of Nod factors by the bacteria which instruct the plant root to prepare for invasion [174]. In the symbiotic association $V$. fischeri-squid described above, bacteria also have a rich interaction with their animal host. For example, it has been found that the bacteria communicate with the host to drive the development of the tissues of the light organ [193]. The biochemical signaling between $V$. fischeri and its host involves both nitric oxide synthase (NOS) and NO [193], which are important components of innate immunity. In addition, the host is capable of detecting the light emitted by the bacteria and selects only luminous symbionts [112].

The QS gene regulatory networks can be broadly classified into two categories: QS systems in Gram-negative bacteria which use acyl homoserine lactones (AHLs) with various carbon chain length (from $\mathrm{C}_{4}$ to $\mathrm{C}_{18}$ ) as signaling molecules, and QS networks in Grampositive bacteria which primarily use modified oligopeptides as autoinducers. The fundamental difference between these two classes of signaling networks lies in the transport and detection modes of the signaling molecules. In many Gram-negative bacteria, small AHLs diffuse freely across the cell membrane [84] and bind specifically to a cytoplasmic receptor protein, such as LuxR in V. fischeri [95], which activates the transcription of target genes. In Gram-positive bacteria, the peptide signals are typically sensed by membrane-bound receptors to initiate a phosphorylation cascade that leads to the activation of target gene expression [121]. Because peptides cannot cross biological membranes, secretion of QS oligopeptides is usually performed by specialized transporter proteins (see also section 2.3.4). Thus, although the general mechanism of QS in Gram-negative and Grampositive bacteria is the same, i.e. the density-dependent expression of target genes via the production, secretion and detection of a signaling molecule, the synthesis of the autoinducer, the secretion and detection apparatus and the signaling molecule itself are different 
[14]. The classification into these two categories is however not exact and some bacterial species may integrate characteristics of regulatory networks of both Gram-negative and Gram-positive bacteria, as in the case of Vibrio harveyi [121].

The LuxI/LuxR system of Vibrio fischeri has become a paradigm for quorum sensing in Gram-negative bacteria and many other species have been found to share a common regulatory motif. The LuxI protein is the autoinducer synthase and is responsible for the production of the QS autoinducer N-3-(oxo-hexanoyl)-homoserine lactone (3OC6HSL) [160]. After synthesis, the autoinducer molecule diffuses freely across the cell membrane [84] and accumulates in the environment. Upon reaching a threshold concentration, the autoinducer is detected by the cytoplasmic receptor LuxR, which binds reversibly to $3 \mathrm{OC} 6 \mathrm{HSL}$ and form a stable complex. The LuxR-3OC6HSL complex then activates transcription of the lux operon by binding to the $l u x$ box in the promoter region [142], leading to the production of proteins responsible for the light emission. Because the expression of luxI is also activated by the LuxR-3OC6HSL complex, more autoinducer molecules are produced and released into the surrounding environment, which bind to LuxR receptors and activate further the QS circuits in all the cells. The resulting positive feedback loop, or autoinduction, is believed to be responsible for the bistable switch-like behavior of the QS circuit (see also paragraph on positive feedback loops on page 13), enhancing the synchrony of the activation of all cells in the population. Such a bistable behavior has been reported in in silico studies $[45,39,62]$ and in vivo experiments [198, 66].

Increasing knowledge of the QS mechanisms that regulate virulence in pathogenic bacterial species has lead to promising new strategies to inhibit virulence gene expression by interfering with QS communication. In principle, the QS communication mechanism could be inhibited by reducing signal production, inhibiting signal reception or accelerating signal degradation. Most of recent studies focus on finding antagonist compounds that strongly bind to the LuxR-type receptors, competing with the native autoinducer and reducing the QS response [94, 161]. 
QS has opened an important research field with promising technological applications [126, 37]. In the field of synthetic biology, QS components have been used to engineer complex genetic devices with novel behaviors. The design, construction and study of synthetic genetic circuits that use QS signaling can facilitate our understanding of collective behavior in natural systems and lead to interesting applications such as biosensors [37]. The LuxI/LuxR system of Vibrio fischeri is one of the most widely used component to build synthetic QS-based networks. In the work of Basu and al. [15] (see figure 1.5 panels A and B), two bacterial strains are engineered: "sender" cells which are inoculated in the center of a Petri dish, produce AHL autoinducer molecules which freely diffuse on the solid agar surface to create a spatial gradient of signaling molecule concentration; "receiver" cells are engineered to express GFP only when the AHL concentration is within a narrow range (similar to a band-pass filter), resulting in a ring pattern of fluorescence. In the experimental study of Liu and al. [104], the LuxI/LuxR QS module is coupled to a gene module controlling the cell motility in E. coli cells, such that motility is suppressed at high cell density. The coupling between cell density and cell motility leads to the formation of periodic stripe patterns during the growth of a colony inoculated at the center of a Petri dish (see figure 1.5 panels C and D). Spatiotemporal pattern formation is one impressive example of collective behavior performed by QS synthetic circuits. The study of QS synthetic and natural systems can therefore provide insights into the more complex spatiotemporal organization mechanisms found in the development of higher organisms. Other examples of QS applications in synthetic biology include programmed population control [201], environmentally controlled invasion of cancer cells [3] and synchronized population of genetic oscillators, at the level of microcolonies [41] and at the macroscopic level [140]. Thus, the QS mechanism offers a fantastic tool for creating gene regulatory circuits with a variety of novel population-level behaviors. The success of these synthetic biology experiments fundamentally relies on the sinergy between experimental work and theoretical analysis. Mathematical modeling can be used not only to test the design of synthetic 
circuits and make predictions, but also to gain a deeper understanding of collective behaviors in populations of communicating cells in their natural context [63]. In this sense, bacterial QS is a model system to study the emergence of multicellular behaviors in prokaryotic and eukaryotic systems.

Figure 1.5 (following page): Examples of spatiotemporal pattern formation in synthetic QS-based circuits. (A) Design of the synthetic bacterial multicellular system of the study of Basu and al. [15]. Sender cells express LuxI, which synthesizes the autoinducer molecule AHL. Diffusion of the autoinducer in the solid medium creates a concentration gradient around the senders. The autoinducer enters the nearby receiver cells and binds to LuxR, activating the expression of $\mathrm{CI}$ and a modified Lac repressor $\left(\mathrm{LacI}_{M 1}\right)$. Due to the design of the regulatory circuit, only receiver cells which receive intermediate levels of autoinducer activate the expression of GFP. (B) This band-pass filter behavior result in a ring pattern of GFP fluorescence when receiver cells are cultivated uniformly in a Petri dish around a colony of sender cells placed in the center expressing CFP. A second strain of receiver cells is cocultivated on the dish, which respond to lower concentrations of autoinducer and express RFP, resulting in a second string pattern of larger diameter. Figures adapted from [15]. (C) Stripe pattern formation in the study of Liu and al. [104]. The density-sensing module is adapted from the core of the LuxR/LuxI QS network. The motility-control module contains the cheZ gene, which was deleted from the cell genome and whose expression is needed for cell motility on semisolid agar. cheZ is expressed at low cell density, leading to the motile phenotype, and repressed by the active LuxR regulator at high cell density, leading to the nonmotile phenotype. (D) The coupling between cell density and cell motility leads to the formation of stripe patterns of cell density variations during the growth of a colony inoculated in the center of a Petri dish. Intricate pattern are formed when placing two inoculums in the middle of the dish. Figures adapted from [104]. 

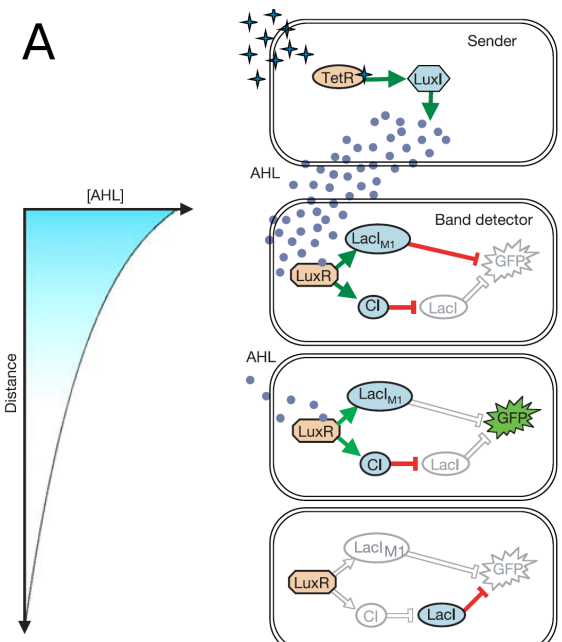

B
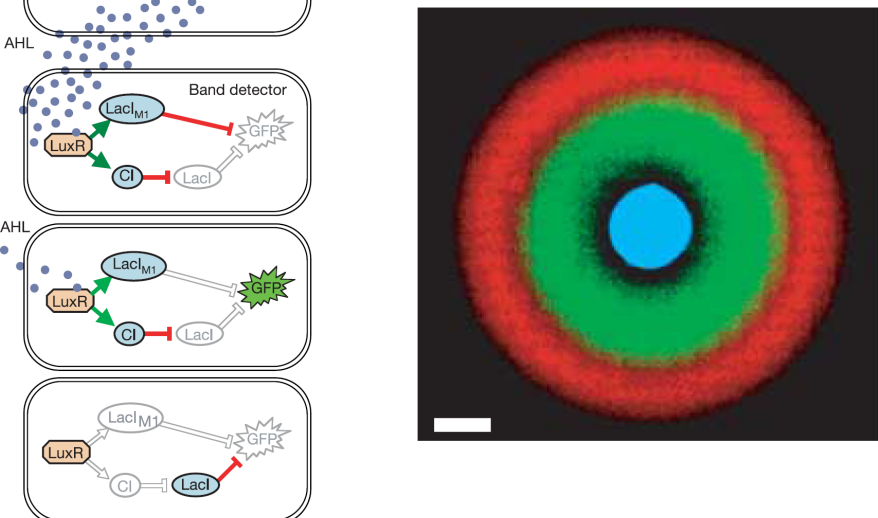

C

Move

D

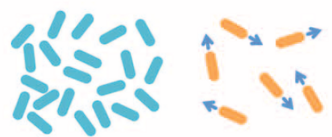

High density Low density
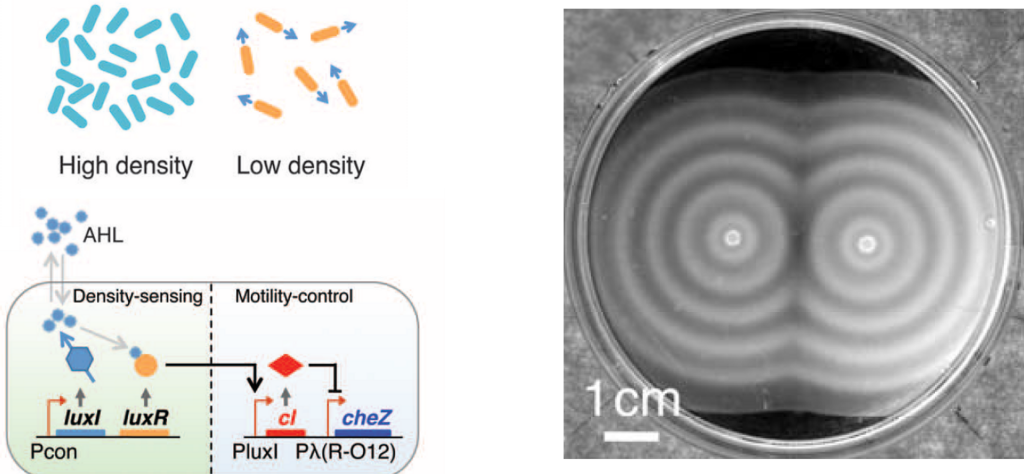


\subsection{STOCHASTIC EFFECTS IN QUORUM SENSING}

The coordination of a myriad of bacterial cells, all expressing the same set of genes in a synchronous manner, may raise the question of how such a coordination can be achieved given the unavoidable fluctuations affecting the biological networks of individual cells. As experiments have shown both in wild-type and synthetic systems (see section 1.2), the QS mechanism can produce a robust and synchronized behavior at the level of the population. As described in section 1.1, noise is ubiquitous in biological systems. Bacterial cells, due to their small size, are particularly subject to intrinsic fluctuations in the number of their regulatory proteins. The core of the QS network may be perturbated, for example, by fluctuations in the level of the LuxR-type receptor. In the recent experimental study of Teng and al. [181], the number of LuxR proteins in Vibrio harveyi was calculated by statistical analysis of protein and volume distributions after cell division. At low cell density the average number of LuxR dimers is between 80 and 135 . This may sound like a large number of proteins, enough to keep intrinsic fluctuations at a low level. However, around 70 target genes are under the control of LuxR, meaning that there are few LuxR dimers relative to the number of operators it can potentially bind to, suggesting that intrinsic noise may have a considerable effect in the regulation of these genes. At high density, the number of LuxR dimers increases to an average of 575 and the average burst size was found to be $b=50$. In other words, around 11 luxR mRNAs are produced per cell cycle and each of these mRNAs translates into 50 LuxR dimers before it is degraded. Such a high translation rate suggests that the gene expression noise may be an important source of stochasticity at high cell density. Thus, intrinsic noise can be expected to play an important role in QS regulatory networks.

As in any communication process, the QS signal may also be perturbated by fluctuations during its transmission from one cell to another. Such extrinsic noise sources could arise for example from spatial heterogeneity in the diffusivity of the external medium, or degradation of the autoinducer by extracellular enzymes [94]. More- 
over, the detection of the freely diffusing signaling molecule is subject to intense molecular noise due to the small volume of the cell and the low concentration of signal in the environment [125]. For a Vibrio fischeri bacterium with a typical volume of $0.35 \mu \mathrm{m}^{3}$ [84], a concentration of autoinducer as low as $10 \mathrm{nM}$ is enough to induce a QS response [84]. This means that a level of only $\sim 2$ signaling molecules per cell can be sensed by the small marine bacterium. The low number of signaling molecules inside the cell, together with the other extrinsic sources of noise, suggest that fluctuations in the autoinducer level may influence the reliability of the QS activation pathway. One central question is therefore to know how the diffusion process influences the intensity of the fluctuations in the signaling molecule (see chapter 2).

As described in section 1.I, at the level of a single cell noise may be controlled and regulated by different mechanisms, such as negative feedback loops. At the level of the population, however, the role of cell-cell communication in the regulation of noise is still poorly understood [63]. An intuitive view is that communication by QS increases cell function's robustness to noise by averaging out the fluctuations in the signal coming from many cells. By acting in a coordinated manner, a population of cells would therefore be able to respond to signals with a higher sensitivity, overcoming the fluctuations that are present in the single cell. In the case of bistable networks, it has been proposed that communication provide a mechanism for enhancing the reliability of collective cellular decision making [91]. Cell-cell communication has also been shown to synchronize synthetic genetic oscillators, as predicted theoretically $[114,53]$ and demonstrated experimentally [41]. However, the interplay between noise and cell-cell communication may in general depend on the details of the genetic network and the communication mechanism. One example showing the complexity of this relation is the case of the suprachiasmatic nucleus (SCN), the master circadian pacemaker in mammals. The SCN is composed of a network of cells, with each cell acting as an autonomous oscillator. Due to the noise in biochemical reactions that perturbates the intracellular clock network, isolated cells are not able to maintain a precise rythm. 
However, in the SCN network, the intercellular coupling allows the emergence of precise and synchronized rhythm in all the cells. This effect has been widely studied and relies on the idea that intercellular coupling can synchronize and improve the precision of noisy oscillators, as shown by experiments [103] and mathematical modeling [61]. In the study of Ko and al. [89], mutant cells that lack one essential component of the intracellular clock regulatory network are analyzed. When isolated, these mutant neurons are unable to produce oscillations and exhibit random fluctuations in the level of the reporter. Surprisingly, mutant cells that are interconnected in a network show stochastic oscillations synchronized over the whole population, similar to the original circadian oscillations but lacking their regularity. In this case, cell-cell coupling and noise work in synergy to generate stochastic rhythms at the level of the cell population. This observation shows how the interplay between intrinsic and extrinsic fluctuations and cell communication can produce complex behaviors that can have relevant biological function, such as circadian oscillations that coordinate the physiological processes of mammalian cells in a daily manner.

\subsection{OVERVIEW OF THE THESIS}

The QS mechanism in prokaryotes is a simple communication process and could serve as a model system for studying the emergence of collective behavior in populations of communicating cells. The interplay between fluctuations and QS communication, however, are still not well understood. Interesting unanswered questions remain about the role of noise in both natural and synthetic QS systems. For example, the cell response heterogeneity observed in many bacterial species (see section 3.1) during the QS transition may originate from different known and unknown noise sources. The diffusion process of the autoinducer could provide a mechanism to regulate the fluctuations inside the cell (see chapter 2). While previous work has suggested that diffusion reduce the noise in the signal level, the interplay between the different noise sources and the diffusion re- 
mains obscure. Moreover, understanding the exact relationship between the stochasticity at the level of a single cell and the reliability of the cell coordination at the level of the population could be important for the design of robust QS-based synthetic circuits (see chapter 5) and to gain insight into the natural function of QS (see chapter 3). At the level of a single cell, many aspects of the effects of stochasticity in gene regulatory networks remain unknown. Beyond the trivial variability in the level of the regulatory proteins, stochasticity can also have more subtle effects, such as changing the position and stability of the stable states of the network (see chapter 4 ). Such effects could be especially relevant in cell differentiation circuits, such as bistable genetic switches, where the stability of the stable states and the stochastic transitions between them are essential characteristics of the cell differentiation process.

In this thesis, we adopt a theoretical scope to analyze these questions. We make use of mathematical modeling and stochastic simulations to study the effects of fluctuations at the level of a single cell and at the level of a population of QS-communicating cells. Our analysis relies on quantitative biological data when available and focus on well-studied canonical experimental models, such as the LuxI/LuxR system. However, the scope of this work is broader and intends to gain a better understanding of the interplay between QS communication and intracellular noise. The modeling presented in this thesis is based on well-established deterministic and stochastic methods (such as the Gillespie algorithm [57]) to describe gene regulatory networks and signaling mechanisms. We make use of a custom-developed software (see appendix A.I) to simulate the stochastic dynamics of a population of cells that can grow and divide, taking into account the stochasticity of the biochemical reactions and the diffusion of the signaling molecule. This simulation tool is used throughout the thesis to study the effects of stochasticity at the level of the single cell and at the level of the cell population.

This thesis is divided into six chapters, the first one being this introduction. The chapters 2, 3, 4 and 5 each describes original research studies. The corresponding publications can be found in the Publications list on page $v$. 
The chapter 2 is dedicated to the analysis of the noise regulation by the QS diffusion process. With the intention of studying the interplay between noise and diffusion from a theoretical point of view, we build a simplified model that describes the expression of the autoinducer and its diffusion in a population of cells. When the transcription rate of the luxI-type gene is very low, we can disregard all the regulation feedback loops and assume that the autoinducer is expressed constitutively. In this regime, we make use of deterministic and stochastic modeling to derive analytically the distribution of autoinducer molecules at steady-state. This theoretical approach allows us to isolate the contributions of different noise sources, such as the transcriptional noise and the biochemical noise. Interestingly, we find that the intensity of fluctuations in the autoinducer level shows a non-monotonic behavior as a function of the diffusion rate, a result that had not been described before. Such analysis contributes to gain a deeper understanding of the interaction between intracellular noise sources and the QS communication process.

In chapter 3, I introduce our study of the stochastic effects in the QS transition of a population of bacteria. The aim of this study is to investigate how intrinsic noise at the level of individual cells affects the coordination of the QS activation at the level of the cell population. How does the collective response depend on the stochastic dynamics of single cells, and in particular, how precise is this response? In order to answer these questions, we build a stochastic multi-cellular model of the well-characterized LuxI/LuxR system. Our results indicate that the cell response is very heterogeneous, in agreement with many recent experimental studies in a variety of QS species $[5,24,136,65]$. We find that the gene expression noise in LuxR is the main factor controlling the variability of the cell response. Our in silico results suggest that noise in the LuxR-type regulator has a large impact on the precision of the QS transition. Thus, we provide with a possible explanation for the existence of LuxR noise control mechanisms found in wild-type bacterial species such as $V$. harveyi [181, 189].

In chapter 4, I present our analysis of the effects of intrinsic noise on the phenotypic landscape of an autoactivating genetic switch. As 
described in section 1.1.4, positive feedback loop is an important gene regulatory motif that can lead to bistability. Such an autoactivating mechanism is at the core of the LuxI/LuxR QS regulatory network of $V$. fischeri and is responsible for the switch behavior of the QS activation. As shown recently in several theoretical studies $[88,162,203,52,79]$, fluctuations can not only change the dynamics of gene regulatory networks but also modify the position of their stable states. In chapter 4 , we make use of analytical calculation and stochastic simulation to show how the epigenetic landscape is modified due to the intrinsic noise. We explain why and how the noise promotes the stability of the low-state phenotype of the switch and show that the bistable region is extended when increasing the intensity of the fluctuations, an effect that we call stochastic stabilization. Our study contributes to a better knowledge of the consequences of biochemical noise in gene regulatory networks and suggests that noise may also redesign the epigenetic landscape of more complex cell differentiation processes.

In chapter 5 , I describe our model of a population of toggle switches interfaced by two QS communication pathways. A synthetic biology study has shown that the QS signaling can induce the flipping of a population of toggle switches [90]. The QS signal, however, acted as a global activator to flip all the switches once to the "on" state, similar to the wild-type density-dependent QS transition. In chapter 5, we present a computational model of a population of toggle switches coupled by two QS signaling pathways such that both the "on" and the "off" states of the switch are coupled. Due to the small size of bacteria, intracellular noise drives the stochastic switching of individual cells. We show that, by increasing the diffusion rate, the cell population transitions from a disordered behavior with the cells randomly flipping from one state to another, to a fully coordinated behavior where all the cells are stuck in the same state. This sudden transition in the coordination of the population appears very similar to the second order phase transition in the mean-field theory of the Ising model. The phenomenon of phase transition suggests a new mechanism for collective cellular decision making, relying on the interplay between noise, bistability and cell-cell coupling. With 
our model, we suggest a new design of QS-based genetic circuit that produces a coordinated and tunable behavior at the level of cell population. By analyzing the response of the system to an external signal, we suggest that this phenomenon could be used to increase the sensitivity and robustness of biological sensors.

Finally, in chapter 6, I present the main achievements of the thesis and discuss future perspectives. 


\subsection{INTRODUCTION}

Communication by quorum sensing (QS) in many gram-negative bacteria relies on the diffusion of the signaling molecule through the cell membrane. The low number of signaling molecules inside the cell, together with other sources of noise, suggest that fluctuations at the autoinducer level may influence the reliability of the QS activation pathway. Therefore, as pointed out in section 1.3, one central question is to elucidate how the diffusion process influences the intensity of the fluctuations in the signaling molecule.

A recent study has indeed shown that diffusion can reduce the noise at the level of the autoinducer [179]. However, the role played by different sources of stochasticity present in the cell and their contribution to the dynamics of the signaling molecule have not been characterized yet. In the study of Tanouchi and al. [179], the authors assume a constitutive expression of the receptor protein (LuxR) and the autoinducer, and neglect the transcriptional noise. Yet, the latter can be an important source of noise, in particular when the transcription rate is low [82]. Moreover, as will be shown in chapter 3, LuxR regulates crucial aspects of QS as the precision for achieving a coordinated response among cells.

In order to study the interplay between different sources of intrinsic noise and the diffusion of the signaling molecule, we propose a model that describes the expression of the autoinducer and its diffusion in a population of cells when the QS genes are not activated. In this case, the fluctuations of the signaling molecule are mainly modulated by the diffusion into and out of the cell. When the autoinducer concentration is below the activation threshold, the tran- 
scription of the luxI gene occurs at a low basal rate, thus producing low levels of the enzyme. In this regime, the feedback regulation of the luxI gene leading to autoinduction can be disregarded. As a matter of fact, a number of QS systems lack autoinduction [146] and in those cases neglecting the feedback is a valid approximation even above the activation threshold.

The QS network architecture varies among species and may include complex regulation mechanisms [121]. At low cell density, the luxI gene expression is either repressed by a high concentration of its repressor or activated at a very low level by its activator. Under these conditions, we assume that very few luxI transcripts will be produced, following rare events of successful transcription. These events may happen when the activator molecule, such as the LuxRautoinducer complex in the case of Vibrio fischeri, binds to its promoter for sufficient time to allow initiation of transcription. In the case of repression, a transcription event may take place when the repressor unbinds from the promoter for a sufficient time, a process known as leaking. Such infrequent transcription events have been observed in the lac operon in E. coli (see section 1.1). Expression of the operon is regulated by the lac repressor, which binds tightly to its promoter region on the DNA. Single molecule observations have shown that infrequent dissociation of the lac repressor produce rare transcription events producing only one mRNA molecule [202, 36]. In our modeling approach we assume that during the transcription events a single luxI mRNA molecule is produced. Under these conditions the dynamics of the mRNA can be then described by means of a Markovian dichotomous process [73], jumping between zero and one molecule. This theoretical description will allow us to derive analytical results for the dynamics of the autoinducer.

\subsection{OBJECTIVES AND SUMMARIZED RESULTS}

In this chapter, I present our study of the interplay between the QS communication and intracellular fluctuations in bacterial populations. First, we aim at understanding how the communication 
mechanism and different sources of noise determine the dynamics of the autoinducer. Second, we aim at shedding light on the mechanisms that confer robustness to noise in QS communication. We restrict ourselves to the study of the aforementioned problems below the QS activation threshold where we can assume that the transcription events of the luxI-type gene produce basal constitutive levels of mRNA of at most one molecule per cell at a time, that the mRNA is translated into the signaling molecule in a single reaction step, and that all regulatory feedback loops can be neglected.

In the Methods section, I present our modeling approach and derive the analytical expression of the autoinducer distribution as a function of the diffusion rate. In the Results section, we compute by means of stochastic simulations the autoinducer distribution. By comparing the analytical and simulations results, we are able to distinguish the contributions of the intrinsic noise and the transcriptional noise in the fluctuations of the autoinducer. We show how the diffusion process produces a repertoire of dynamics in regards of the signaling molecule. We demonstrate that, for a large range of diffusion rate values, the main contribution to the total noise of the autoinducer concentration are the mRNA fluctuations. Finally, we show that the total noise exhibits a non-monotonic behavior as a function of the diffusion rate in contrast to previous results [179].

\subsection{METHODS}

\subsubsection{Modeling Approach}

As discussed in section 2.1, below the activation threshold, it can be assumed that rare transcription events produce individual luxI transcripts. Under these conditions the dynamics of the mRNA can be then described by means of a Markovian dichotomous process [73],

$$
M_{0}^{i} \underset{\alpha}{\stackrel{\beta}{\rightleftarrows}} M_{1}^{i}
$$


where $M_{0,1}^{i}=0,1$ stands for the number of mRNA molecules at cell $i$ and $\alpha$ and $\beta$ for the transition rates between these states; i.e. $\alpha$ and $\beta$ account for the mRNA degradation rate and the transcription frequency respectively. Notice that the fluctuations of the mRNA dynamics between the values o (no mRNA) and I (a mRNA molecule) are not memoryless, i.e. white. Once a mRNA molecule is produced, and until it becomes degraded, the cell keeps producing the autoinducer. That is, the transcriptional noise is a colored noise, and its autocorrelation decays exponentially with a characteristic time scale $\tau_{\mathrm{c}}=(\alpha+\beta)^{-1}[73]$.

Once a mRNA molecule is produced the translational, and posttranslational processes (if any), leads to the appearance of functional luxI synthetases. Yet, our interest here focuses on the dynamics of the signaling molecule. It has been shown that the amount of the synthetase substrate is not a limiting factor for the production of the autoinducer $[117,127]$. As a consequence, the levels of the signaling molecule depends directly on the expression levels of the synthetase. Ignoring intermediate biochemical steps in the autoinducer synthesis reduces the number of noise sources and may even change, under some circumstances, the observed dynamics [166]. Still, we assume here that the translation of the synthetase and the subsequent synthesis of the autoinducer, $A$, can be effectively described by a single chemical step with rate $k_{+}$. In addition, we consider that the autoinducer becomes degraded at a rate $k_{-}$, that is,

$$
\begin{aligned}
& M_{1}^{i} \stackrel{k_{+}}{\longrightarrow} M_{1}^{i}+A^{i} \\
& A^{i} \stackrel{k_{-}}{\longrightarrow} \varnothing .
\end{aligned}
$$

Passive diffusion in and out the cell of the autoinducer can be implemented by considering a new species, $A_{\text {ext }}$, that accounts for the number of signaling molecules in the extracellular medium such that

$$
A^{i} \underset{r D}{\stackrel{D}{\rightleftarrows}} A_{\text {ext }}
$$

where $\mathrm{D}$ stands for the diffusion rate and $r=V / V_{\text {ext }}$ represents the ratio of the volume of a cell to the total extracellular volume. 
We consider all cells to have the same value of the diffusion rate. In addition, we assume a well-stirred system where spatial effects can be neglected. As the bacterial population grows the autoinducer accumulates in the media. In experiments, in order to keep the concentration of the autoinducer below the activation threshold, such growth is compensated by means of a dilution protocol. As detailed below (see Parameters) the latter constitutes the main source of effective degradation of the signaling molecule. Thus, hereinafter we take for granted the degradation rate of the signaling molecule to be the same inside and outside the cell,

$$
A_{\text {ext }} \stackrel{\mathrm{k}_{-}}{\longrightarrow} \varnothing .
$$

Figure 2.I schematically represents the biochemical processes considered in our approach. The set of reactions reactions 2.1 to 2.5 characterizes the stochastic dynamics of the autoinducer and the mRNA. Their probabilistic description is given by the corresponding master equations that can be sampled by means of the Gillespie algorithm in a N-cells system [57].

\subsubsection{Analytical Calculations: Null Intrinsic Noise Approximation}

Further insight into the dynamics of the signaling molecule can be obtained by analytical means as follows. Two stochastic contributions drive the dynamics of $A$ : the mRNA fluctuations due to the random switching and the molecular, i.e. intrinsic, noise due to low copy number of the autoinducer. As of the latter it can be neglected if over the course of time $A^{i} /\left(A^{i}+1\right) \simeq 1$ (large number of autoinducer molecules). While in this system such approximation is not totally justified (see parameters values below), it is useful to implement it in order to discriminate between the effects caused by different stochastic contributions and to obtain analytical expressions. In this case it is straightforward to demonstrate that the dynamics of 


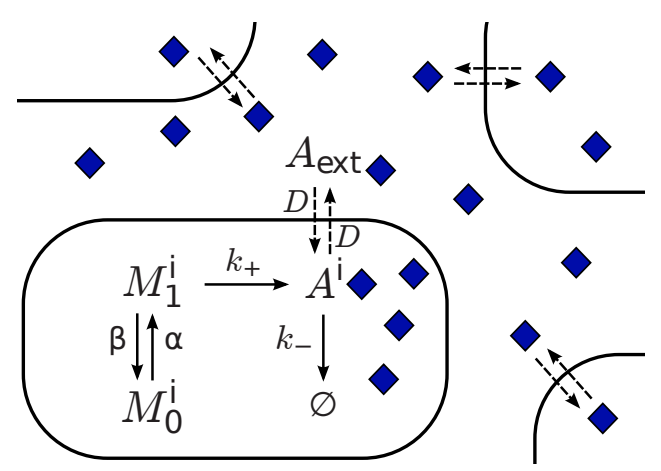

Figure 2.1: Scheme of a simplified biochemical network of QS systems near the activation threshold. Schematic representation of the biochemical processes considered in our approach for describing the dynamics of the signaling molecule, $A$ (diamonds), in cell $i$. The mRNA dynamics follow a dichotomous process with state values $M_{0,1}$ corresponding to zero and one molecule respectively. Once the autoinducer has been produced, it can diffuse into and out of the cell leading to cell communication (see text).

the autoinducer, reactions 2.1 to 2.5 , can be described by the following coupled stochastic equations:

$$
\begin{aligned}
\dot{c}_{A^{i}} & =k_{+} c_{M_{1}^{i}}(t)-k_{-} c_{A^{i}}+D\left(c_{A_{e x t}}-c_{A^{i}}\right) \\
\dot{c}_{A_{e x t}} & =-k_{-} c_{A_{e x t}}+r D \sum_{i=1}^{N}\left(c_{A^{i}}-c_{A_{e x t}}\right)= \\
& =-k_{-} c_{A_{e x t}}+\operatorname{rDN}\left(\left\langle c_{A}\right\rangle-c_{A_{e x t}}\right),
\end{aligned}
$$

where $c_{A^{i}}=A^{i} / V, c_{M_{1}^{i}}(t)=M_{1}^{i} / V$, and $c_{A_{\text {ext }}}=A_{\text {ext }} / V_{\text {ext }}$ stand for the concentration of species $A$ and $M_{1}^{i}$ in cell $i$ and for species $A_{\text {ext }}$ in the extracellular medium respectively, $N$ is the colony size, and $\langle\cdot\rangle$ represents the population average. In equation (2.6) the term $c_{M_{1}^{i}}(t)$ accounts for a dichotomous stochastic process characterized by the rates and states $(\alpha, \beta)$ and $(0,1 / V)$ respectively, and describes the fluctuating mRNA dynamics. Notice that if $\mathrm{D}=0$ then the resulting equation from equation (2.6) has been proposed to study graded 
and binary responses in stochastic gene expression and it has been shown that, despite its simplicity, it can actually reproduce some gene expression phenomena [86, 188, 77].

We can further proceed with the analytical calculations by implementing, as in previous studies e.g. [114], a quasi-steady approximation for the dynamics of the external autoinducer, i.e. $\dot{c}_{A_{\text {ext }}}=0$. Notice that this approximation is justified by the fact that the steadystate dynamics of $c_{A_{\text {ext }}}$ are determined by the average of a large number of fluctuating variables $c_{A^{i}}$. As we will show in this study, the main source of randomness in $c_{A^{i}}$ originates in the dynamics of the mRNA. The fluctuations in the mRNA are uncorrelated between cells and therefore we can expect the dynamics of $c_{A^{i}}$ to be approximately independent between cells. Moreover, we consider that all cells are identical and therefore the steady-state probability distributions of $c_{A^{i}}$ are identical. Due to the central limit theorem, we can expect $c_{A_{\text {ext }}}$ to be a constant as a first approximation,

$$
c_{A_{\text {ext }}}=\left\langle c_{A}\right\rangle \frac{1}{1+\frac{k_{-}}{N D r}} .
$$

By substituting equation (2.8) into equation (2.6) we obtain a rate equation for the concentration of the signaling molecule inside a given cell that depends on the average $\left\langle c_{A}\right\rangle$ (the index $i$ has been dropped),

$$
\dot{\mathrm{c}}_{\mathrm{A}}=\mathrm{k}_{+} \mathrm{c}_{\mathrm{M}_{1}}(\mathrm{t})-\mathrm{D}\left(1+\frac{\mathrm{k}_{-}}{\mathrm{D}}\right) \mathrm{c}_{\mathrm{A}}+\left\langle\mathrm{c}_{\mathrm{A}}\right\rangle \frac{\mathrm{D}}{1+\frac{\mathrm{k}_{-}}{\mathrm{NDr}}} .
$$

In the absence of diffusion, equation (2.9) reveals that the concentration of the signaling molecule reaches a maximum value of $c_{A}^{+}=$ $k_{+} /\left(k_{-} V\right)$ when $c_{M_{1}}(t)=V^{-1}$. In terms of $c_{A}^{+}$and the time scale $t_{c}=1 / k_{-}$(the typical lifetime of a signaling molecule), the dimensionless version of equation (2.9) reads

$$
\dot{\tilde{\mathbf{c}}}_{\mathrm{A}}=\hat{\mathrm{c}}_{\mathrm{M}_{1}}(\tilde{\mathrm{t}})+\mathrm{k}_{+}^{\mathrm{eff}}\left(\left\langle\tilde{\mathbf{c}}_{\mathrm{A}}\right\rangle\right)-\mathrm{k}_{-}^{\mathrm{eff}} \tilde{\mathrm{c}}_{\mathrm{A}},
$$


where

$$
\begin{aligned}
& \widetilde{\mathrm{D}}=\mathrm{D} / \mathrm{k}_{-} \\
& \mathrm{k}_{-}^{\mathrm{eff}}=1+\widetilde{\mathrm{D}} \\
& \mathrm{k}_{+}^{\mathrm{eff}}\left(\left\langle\tilde{\mathrm{c}}_{\mathrm{A}}\right\rangle\right)=\left\langle\tilde{\mathrm{c}}_{\mathrm{A}}\right\rangle \frac{\widetilde{\mathrm{D}}}{1+\frac{1}{\mathrm{~N} \widetilde{\mathrm{D} r}}} ;
\end{aligned}
$$

$\hat{\mathbf{c}}_{M_{1}}(\tilde{\mathrm{t}})$ being a Markovian dichotomous noise with states $\left\{\hat{\boldsymbol{c}}_{M_{1}}\right\}=$ 0,1 and rates $\widetilde{\alpha}=\alpha / k_{-}$and $\widetilde{\beta}=\beta / k_{-}$.

Equation (2.10) can be formally closed by invoking the following self-consistency condition:

$$
\left\langle\tilde{\mathbf{c}}_{A}\right\rangle=\int_{\widetilde{\Omega}} \tilde{\mathbf{c}}_{A} \rho\left(\tilde{\mathbf{c}}_{A} ;\left\langle\tilde{\mathbf{c}}_{A}\right\rangle\right) \mathrm{d} \tilde{\mathbf{c}}_{A}
$$

$\rho\left(\tilde{\mathbf{c}}_{A} ;\left\langle\tilde{\mathbf{c}}_{A}\right\rangle\right)$ being the probability density solving equation (2.10) and $\widetilde{\Omega}$ its support (see below) [73]:

$$
\begin{aligned}
\rho\left(\tilde{\mathbf{c}}_{A} ;\left\langle\widetilde{\mathbf{c}}_{A}\right\rangle\right)=\mathcal{N}\left(k_{-}^{\text {eff }} \tilde{\mathbf{c}}_{A}-k_{+}^{\text {eff }}\left(\left\langle\tilde{\mathbf{c}}_{A}\right\rangle\right)\right)^{\frac{\tilde{\beta}}{k_{-}^{\text {eff }}}-1} \\
\left(1+k_{+}^{\text {eff }}\left(\left\langle\tilde{\mathbf{c}}_{A}\right\rangle\right)-k_{-}^{\text {eff }} \tilde{\mathbf{c}}_{A}\right)^{\frac{\widetilde{\alpha}}{k_{-}^{\text {eff }}}-1},
\end{aligned}
$$

with

$$
\mathcal{N}=\frac{(1+\widetilde{\mathrm{D}}) \Gamma\left[\frac{\widetilde{\alpha}+\widetilde{\beta}}{1+\widetilde{\mathrm{D}}}\right]}{\Gamma\left[\frac{\widetilde{\alpha}}{1+\widetilde{\mathrm{D}}}\right] \Gamma\left[\frac{\widetilde{\beta}}{1+\widetilde{\mathrm{D}}}\right]}
$$

being the normalization constant. The condition (2.14) can be exactly solved and leads to the following value for the average concentration:

$$
\left\langle\tilde{\mathrm{c}}_{A}\right\rangle=\frac{1+\widetilde{\mathrm{D} N} r}{1+\widetilde{\mathrm{D} N} r+\widetilde{\mathrm{D}}} \frac{\widetilde{\beta}}{\widetilde{\alpha}+\widetilde{\beta}}=\left.\frac{1+\widetilde{\mathrm{DN}} r}{1+\widetilde{\mathrm{D} N} r+\widetilde{\mathrm{D}}}\left\langle\widetilde{\mathrm{c}}_{A}\right\rangle\right|_{\widetilde{\mathrm{D}}=0}
$$

where $\left.\left\langle\widetilde{\mathbf{c}}_{A}\right\rangle\right|_{\widetilde{D}=0}=\widetilde{\beta} /(\widetilde{\alpha}+\widetilde{\beta})$ is the average concentration of the signaling molecule in the absence of diffusion. When the diffusion 
is non zero, the autoinducer is produced in the cell and diffuses out into the external medium where it degrades, leading to a decrease in the overall concentration of autoinducer in the cells, $\left\langle\tilde{\mathbf{c}}_{A}\right\rangle<$ $\left.\left\langle\tilde{\mathrm{c}}_{A}\right\rangle\right|_{\tilde{D}_{=0}}$. For the sake of concision, on what follows we drop, in the notation of $\rho\left(\tilde{\mathfrak{c}}_{\mathcal{A}} ;\left\langle\tilde{\mathfrak{c}}_{A}\right\rangle\right)$, the term $\left\langle\tilde{\mathfrak{c}}_{A}\right\rangle$ from the argument. Note that $\rho\left(\tilde{c}_{A}\right)$ has two states (barriers) that define its support. That is, the minimum and maximum values that the concentration of the autoinducer can reach as a function of the diffusion are:

$$
\begin{aligned}
\tilde{c}_{A}^{-} & =\frac{\widetilde{D}^{2} \mathrm{Nr}}{(1+\widetilde{\mathrm{D}})(1+\widetilde{\mathrm{D}}+\widetilde{\mathrm{D} N r})} \frac{\widetilde{\beta}}{\widetilde{\alpha}+\widetilde{\beta}} \\
\tilde{\mathrm{c}}_{A}^{+} & =\widetilde{\mathrm{c}}_{A}^{-}+\frac{1}{1+\widetilde{\mathrm{D}}} .
\end{aligned}
$$

It is easy to prove that the probability density $\rho\left(\tilde{\mathfrak{c}}_{\mathcal{A}}\right)$ shows a single extremum if

$$
\widetilde{\alpha}, \widetilde{\beta} \lessgtr k_{-}^{\text {eff }},
$$

where the extremum is a maximum if $\widetilde{\alpha}, \widetilde{\beta}>k_{-}^{\text {eff }}$ and a minimum

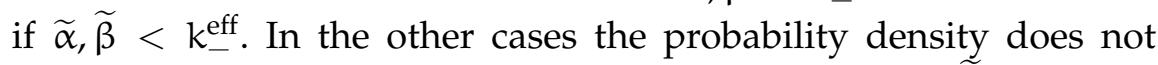
display any extrema. Therefore, as a function of $\widetilde{\alpha}$ and $\widetilde{\beta}$, the probability density $\rho\left(\tilde{\mathbf{c}}_{\mathcal{A}}\right)$ may show four different behaviors depending on the value of the diffusion coefficient as schematically represented in figure 2.2A. However, given the constraints on the parameters of our modeling not all regions, i.e. behaviors, are accessible to the autoinducer dynamics. In particular, we have assumed a low constitutive expression such that only a single mRNA molecule can be transcribed at a time. The latter implies that $\widetilde{\beta}<\widetilde{\alpha}$ (the degradation rate of the mRNA is larger than the transcription rate) in order to assure that a maximum of one mRNA molecule is present in a cell at any given time (see Parameters section 2.3.3). As a consequence, and independently of the diffusion value, the dynamics leading to the probability density shown at the top-left region of figure 2.2A (for which $\widetilde{\beta}>\widetilde{\alpha}$ ) can not be considered as physical in the context of our modeling approach. Yet this constraint is not a fundamental ingredient for obtaining our results (see section 2.5). 
A

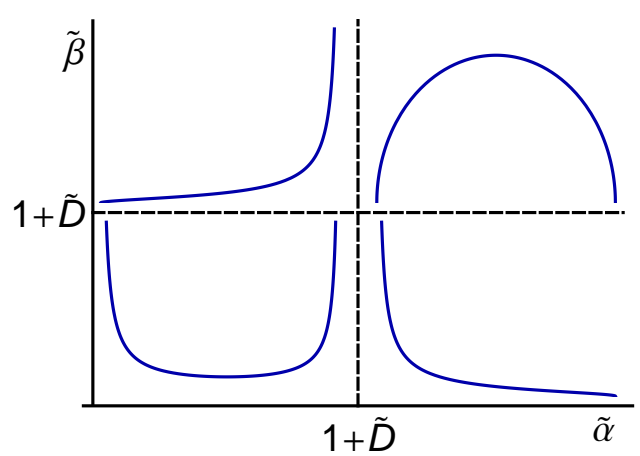

B

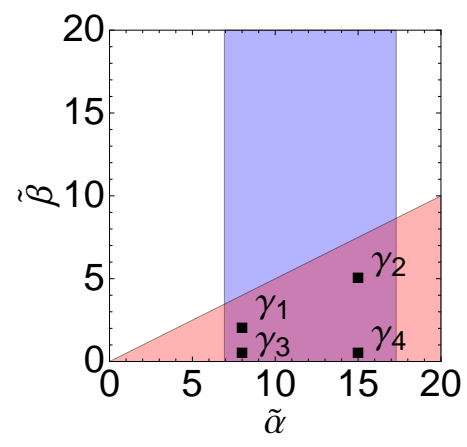

Figure 2.2: Probability densities of the signaling molecule and parameter space. (A) Sketch of the different probability densities of the autoinducer concentration depending on the value of $\widetilde{\alpha}$ and $\widetilde{\beta}$ with respect to $\widetilde{D}$. Given a set of values $(\widetilde{\alpha}, \widetilde{\beta})$ the dynamics of the autoinducer show different behaviors depending on the value of the diffusion parameter. The limits of the parameter regions are located at $\widetilde{\alpha}, \widetilde{\beta}=1+\widetilde{D}$. The constraints of our modeling in terms of the parameter values make the region on the topleft corner non-accessible (see text). (B) Parameter space diagram $(\widetilde{\alpha}, \widetilde{\beta})$ indicating the sets of parameters used in the simulations (solid squares): $\gamma_{1}=(8,2), \gamma_{2}=(15,5), \gamma_{3}=(8,0.5)$, $\gamma_{4}=(15,0.5)$. The experimental values reported for the degradation rate of the mRNA leads to a biological meaningful range for $\widetilde{\alpha}$ (blue region). The low constitutive expression assumption is prescribed by the constraint $\widetilde{\alpha}>2 \widetilde{\beta}$ (red colored region). 
Finally, the noise of the autoinducer concentration reads

$$
\eta_{\tilde{\mathbf{c}}_{A}}^{2}=\frac{\sigma_{\tilde{c}_{A}}^{2}}{\left\langle\tilde{\mathrm{c}}_{A}\right\rangle^{2}}=\frac{\widetilde{\alpha}(1+\widetilde{D}+\widetilde{D} N r)^{2}}{\widetilde{\beta}(1+\widetilde{D})(1+\widetilde{D} N r)^{2}(1+\widetilde{D}+\widetilde{\alpha}+\widetilde{\beta})}
$$

where $\sigma_{\tilde{\mathfrak{c}}_{A}}^{2}=\left\langle\tilde{\mathbf{c}}_{A}^{2}\right\rangle-\left\langle\tilde{\mathbf{c}}_{A}\right\rangle^{2}$. While in the analytical calculations we have disregarded the intrinsic noise, equation (2.21) will allow us to elucidate the contributions of different sources of noise. By means of the numerical simulations (Gillespie) of the set of reactions 2.I to 2.5 in a N-cell system, we can evaluate the total (intrinsic + transcriptional) noise. Hence, by subtracting from that quantity the contribution of the transcriptional noise, i.e. equation (2.21), we obtain the levels of intrinsic noise (see Results).

\subsubsection{Parameters}

We are particularly interested in the role played by the fluctuations of the signaling molecule, $A$, when its concentration is close to the activation of the QS switch. Therefore, we fix the mean concentration of the autoinducer and modulate the rest of the parameters in order to keep constant this value. Pai and You [125] have recently studied the core architecture of the QS mechanism for a comprehensive set of bacterial species and estimated that the critical concentration of autoinducer needed for the activation of the QS genes ranges from 10 to $50 \mathrm{nM}$ for most of the bacterial species. In our model, we set the average concentration of $A$ to a typical value of $c_{A}^{0}=25 \mathrm{nM}$. Yet, our results do not depend on the particular value we choose within that range. As shown below (see section 2.4), this value fixes the level of intrinsic noise of the system. However, the interplay between diffusion and transcriptional noise does not depend on that. Moreover, by defining the so-called sensing potential, $v=(r N)^{-1}$, Pai and You estimated the range of critical cell densities for the QS activation and concluded that its typical value is $v \sim 10^{3}-10^{4}$. In our simulations we set this parameter to $v=10^{3}$. In the experimental setups the cells are typically present in a volume of a few milliliters and 
the total number of cells is of the order of $10^{8}-10^{9}$. The concentration of autoinducer in the medium is therefore determined by the exchange of signaling molecules coming from many cells. In contrast, the behavior of QS systems with a very low number of cells can be significantly different, as shown by microfluidic confinement of cells in picoliter droplets $[24,33,65]$. In our study, in order to discard small system size effects, we choose a sufficiently large number of cells in the numerical simulations, $\mathrm{N}=10^{2}$. Since the typical volume of an E. coli cell is $V=1.5 \mu \mathrm{m}^{3}$, then $V_{\text {ext }}=v N V=10^{5} \mathrm{~V}$ (i.e. $\mathrm{r}=10^{-5}$ ).

We point out that keeping $v$ to a constant value necessarily requires an external dilution protocol for maintaining constant the cell density. The control of the dilution rate is usually achieved by the use of chemostats or microfluidic devices [19]. The rate of dilution should compensate for the cell growth, $\sim 2 \cdot 10^{-2} \mathrm{~min}^{-1}$ (i.e. cell cycle duration $\sim 50 \mathrm{~min})$. In our modeling, by keeping constant the number of cells and the average concentration of the autoinducer, we tacitly assume a dilution protocol too. Importantly, the dilution rate effectively modifies the degradation rate of the signaling molecule. Generally speaking, bacteria which synthesize AHLs do not degrade them enzymatically. In fact, AHLs are chemically stable species in aqueous solutions [87]. However, some bacteria species possess hydrolytic enzymes that do degrade AHLs. In this regard, the degradation rate of the autoinducer has been measured in vitro for the homoserine lactone 3-Oxo-C6-AHL and it has been found that this autoinducer is rather stable: $2.86 \cdot 10^{-4} \mathrm{~min}^{-1}$ [87]. Measurements of the degradation rate of other AHL autoinducers show similar results. Based on experimental data and mathematical modeling, the degradation rate of the signaling molecule in vivo has been also estimated [125]. Depending on the $\mathrm{pH}$ of the medium, the latter ranges from $\sim 5 \cdot 10^{-3} \mathrm{~min}^{-1}$ to $\sim 2 \cdot 10^{-2} \mathrm{~min}^{-1}$. Consequently, the dilution process constitutes the main source of effective degradation of $A$, both inside and outside the cell, and here we set $k_{-}=2 \cdot 10^{-2} \mathrm{~min}^{-1}$.

In regards to the mRNA dynamics, $\alpha$, the degradation rate, depends on the cell's degradative machinery. To this respect, the halflives of all mRNAs of Staphylococcus aureus have been recently mea- 
sured during the mid-exponential phase. Most of the transcripts $(90 \%)$ have half-lives shorter than 5 minutes [150, 4]. According to these studies we restrict the mRNA degradation rate to the range $\ln (2) / 5 \min ^{-1}<\alpha<\ln (2) / 2 \min ^{-1}$ and consequently $\widetilde{\alpha}>1$. As for the frequency of the transcription events, $\beta$ is determined by particular characteristics of the gene regulatory process under consideration, like the affinity of the regulatory proteins to the operator site and the initiation rate of transcription. Due to the assumption of low constitutive transcription, we choose values of parameter $\beta$ satisfying the relation $\alpha>\beta$. In particular in our simulations we implement the more restrictive condition $\alpha>2 \beta$. For all the chosen parameter values, we show that our modeling is compatible with the translational burst theory (see section 2.3.5). Figure 2.2B recapitulates these constraints and shows the different sets of $\alpha$ and $\beta$ values that we have used in our simulations and analytical calculations.

Summarizing, $N, r$, and $k_{-}$are kept fixed in our simulations and analytical calculations and we explore the parameter space $\alpha, \beta$, and $\mathrm{D}$ within the ranges and constraints mentioned above. In every particular situation, once a set of those parameters is prescribed, we determine the value of $k_{+}$by using equation (2.17) in order to keep the average value of $c_{A}$ at a fixed value (25nM), as shown in figure 2.3.

\subsubsection{Passive diffusion and active transport in QS}

The rate of passive diffusion has been estimated for the 3-Oxo-C6AHL autoinducer based on the measure of diffusion of glucose and lactose through the outer membrane of E. coli [179]. For a typical cell volume of $1.5 \mu \mathrm{m}^{3}$ the estimated diffusion rate is $\sim 10^{3} \mathrm{~min}^{-1}$. Under these conditions the typical value for the normalized parameter $\widetilde{\mathrm{D}}$ is of the order of $10^{4}$. Yet, active transport mechanisms for the autoinducer leads to much smaller effective diffusion values and we explore the role played by this parameter.

In some QS systems the autoinducer is actively transported in and out of the cell. For example, in the bacterial species Pseudomonas 

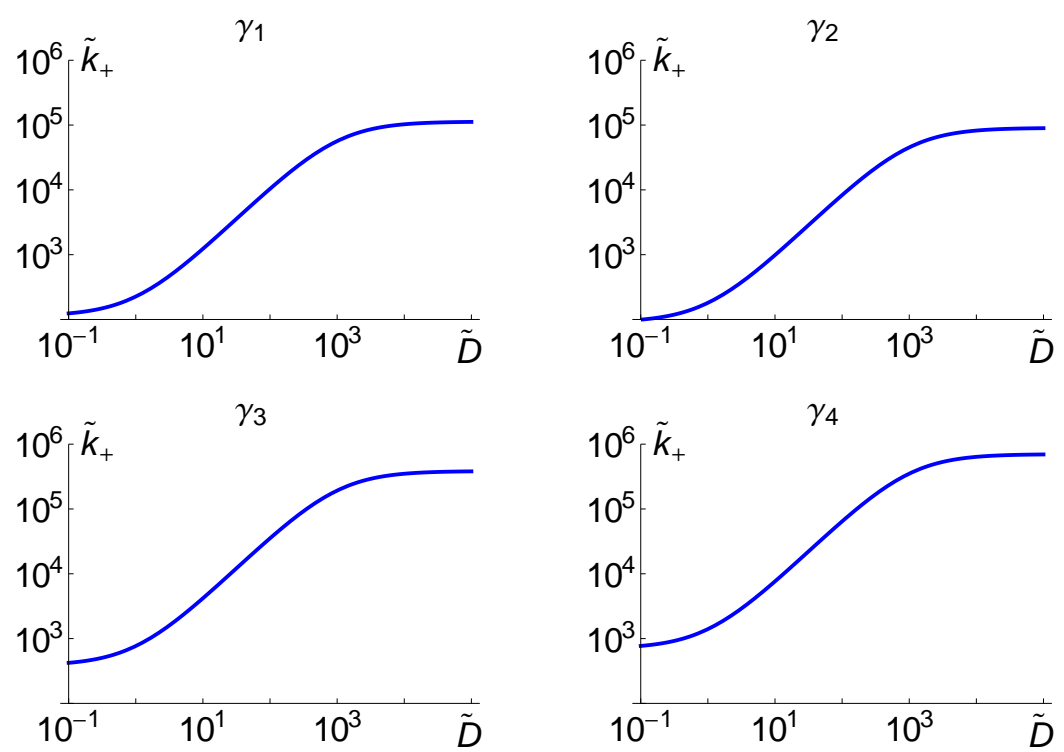

Figure 2.3: Tuning of parameter $\tilde{\mathrm{k}}_{+}$as a function of the diffusion coefficient. Parameter $\tilde{k}_{+}$as a function of diffusion coefficient $\widetilde{D}$ for all the sets of parameters $\gamma_{1,2,3,4}$ (see figure 2.2B). For every value of parameter set $(\widetilde{\alpha}, \widetilde{\beta}, \widetilde{D})$ we adjust the value of $\tilde{k}_{+}=k_{+} / k_{-}$by using equation (2.17) in order to keep the average value of $c_{A}$ at a fixed concentration value $(25 \mathrm{nM})$. 
aeruginosa, C4-HSL can freely diffuse but C12-HSL, a larger signaling molecule, is subjected to active influx and efflux where its importation and exportation rate are of the order of $\sim 10^{-2} \mathrm{~min}^{-1}$ and $\sim 10^{-1} \mathrm{~min}^{-1}$ respectively [131]. Another example corresponds to the AI-2 signaling molecule. The latter is present in many Grampositive and Gram-negative species and it is believed to allow for interspecies communication [200]. In E. coli and Salmonella enterica extracellular AI-2 accumulates during the exponential phase, but then decreases drastically upon entry into the stationary phase. This reduction is due to the import and processing of AI-2 by the Lsr transporter [200, 195]. Moreover, excretion from the cell of this autoinducer also appears to be an active process involving the putative transport protein YdgG (or alternatively named TqsA) [69]. In the case of E. coli these rates have been estimated by computational and experimental means: $D_{\text {out }} \simeq 10^{-1} \mathrm{~min}^{-1}$ and $D_{\text {in }} \simeq 10^{-3}-10^{-2}$ $\min ^{-1}$ [10o]. All in all, the transport rates when driven by active processes are four orders of magnitude smaller than the diffusion rate of small molecules through the membrane. Hence, transport rates in QS systems can be categorized into two main, well separated, classes: small transport rates due to active process, and large diffusion rates due to passive mechanisms.

In principle our model does not account for active diffusion processes, but transport driven by concentration differences. Still, our simple model is valid when the transport mechanism can be described by two symmetric first-order transport reactions. We assume that the excretion and uptake systems follow the Michaelis-Menten kinetics. In the regime where the concentration of autoinducer (substrate) is much smaller than the $\mathrm{K}_{\mathrm{m}}$ of the enzymatic reaction, the transport rate can be approximated by a first-order reaction with rates $D_{i n} c_{A_{\text {ext }}}$ and $D_{\text {out }} c_{a}$. If, in addition, we assume that the transport rates are symmetric, $D_{i n}=D_{o u t}$, the resulting dynamics are identical to the case of passive diffusion. Under these conditions, the rates of active transport in the QS systems described above would fit in our model with a normalized diffusion coefficient in the range $\widetilde{\mathrm{D}} \in\left[10^{-1}, 10\right]$. 
2.3.5 Validity of the low mRNA copy number approximation in the framework of protein burst expression theory

In our analysis, we assume that the rate of transcription is very low, such that one mRNA molecule is present in the cell at most. This assumption allows us to reduce the stochastic dynamics of the mRNA to a dichotomous process that transitions between $o$ and $I$ molecule. We expect the dichotomous approach to describe accurately the mRNA dynamics when the transcription rate is much smaller than the mRNA degradation rate, i.e. $\beta \ll \alpha$. In this case, the probability of finding two mRNA molecules at any given time in the cell is small and the simple dichotomous process is a good approximation of the mRNA dynamics.

The low copy number of mRNA may lead to burst-like production of proteins (see also section 1.1). Single molecule protein experiments $[202,30]$ have shown that genes under the tight repression of the lac promoter are expressed in bursts. Stochastic dissociation events of the Lac repressor from the promoter lead to the transcription of a single mRNA which is translated into a few copies of the protein before the mRNA is degraded. The sudden production of several copies of the protein is referred to as burst-like production. By assuming that the expression events are temporally uncorrelated and that the number of proteins produced per burst is exponentially distributed, the steady-state protein distribution can be described by the following gamma distribution $[51,30]$ :

$$
p_{\Gamma}(x)=\frac{1}{b^{a} \Gamma(a)} x^{a-1} e^{-x / b}
$$

where $a=\beta / k_{-}$is the mean number of bursts per protein lifetime, and $b=k_{+} / \alpha$ the mean number of proteins produced per burst. We show below that the distribution (2.15) derived in our analytical calculations is a good approximation of this gamma distribution when $\beta \ll \alpha$. 
When $\widetilde{\mathrm{D}}=0$, the dimensionless steady-state distribution (2.15) reduces to

$$
\rho_{\operatorname{dich}}\left(\tilde{\mathbf{c}}_{A}\right)=\frac{\Gamma[\widetilde{\alpha}+\widetilde{\beta}]}{\Gamma[\widetilde{\alpha}] \Gamma[\widetilde{\beta}]}\left(\tilde{\mathbf{c}}_{A}\right)^{\widetilde{\beta}-1}\left(1-\tilde{\mathbf{c}}_{A}\right)^{\widetilde{\alpha}-1}
$$

with

$$
\tilde{\mathrm{c}}_{\mathrm{A}}=\frac{\mathrm{c}_{\mathrm{A}}}{\mathrm{c}_{\mathrm{A}}^{+}}=\mathrm{c}_{\mathrm{A}} \frac{k_{-}}{k_{+}}
$$

and support $[0,1]$. We denote this distribution by $\rho_{\text {dich }}$ to emphasize the underlying assumption of the mRNA following a dichotomous process. In order to compare the distribution we obtain in our model to the gamma distribution, we transform $\rho_{\text {dich }}$ back to dimensional variables,

$$
\rho_{\text {dich }}\left(c_{A}\right)=\left|\frac{1}{c_{A}^{+}}\right| \frac{\Gamma\left[(\alpha+\beta) / k_{-}\right]}{\Gamma\left[\alpha / k_{-}\right] \Gamma\left[\beta / k_{-}\right]}\left(\frac{c_{A}}{c_{A}^{+}}\right)^{\frac{\beta}{k_{-}}-1}\left(1-\frac{c_{A}}{c_{A}^{+}}\right)^{\frac{\alpha}{k_{-}}-1}
$$

with support $\left[0, c_{A}^{+}\right]$. In the case $\beta \ll \alpha$, the first moment of this distribution is the same as the mean of the gamma distribution (2.22),

$$
\left\langle c_{A}\right\rangle_{\text {dich }}=c_{A}^{+} \frac{\beta}{\alpha+\beta} \stackrel{\beta}{\approx} \alpha{ }^{+} c_{A}^{+} \frac{\beta}{\alpha}=a b=\left\langle c_{A}\right\rangle_{\text {gamma }} .
$$

Moreover, the second moment, in the case that $\beta \ll \alpha$ and $\alpha \gg 1$, is the same as the second moment of the gamma distribution,

$$
\begin{aligned}
\left\langle c_{A}^{2}\right\rangle_{\text {dich }}=c_{A}^{+} \frac{\beta(1+\beta)}{(\alpha+\beta)(1+\alpha+\beta)} \stackrel{\beta \ll \alpha}{\approx}\left(\frac{c_{A}^{+}}{\alpha}\right)^{2} \frac{\alpha \beta(1+\beta)}{1+\alpha} \\
\quad \stackrel{2}{\approx}\left(\frac{c_{A}^{+}}{\alpha}\right)^{2} \beta(1+\beta)=\left\langle c_{A}^{2}\right\rangle_{\text {gamma }} .
\end{aligned}
$$

We can see in figure 2.4, that the theoretical distribution (2.25) is a good approximation of the gamma distribution when $\beta \ll \alpha$ for the parameter sets $\gamma_{3}$ and $\gamma_{4}$ of our simulations and in qualitative 

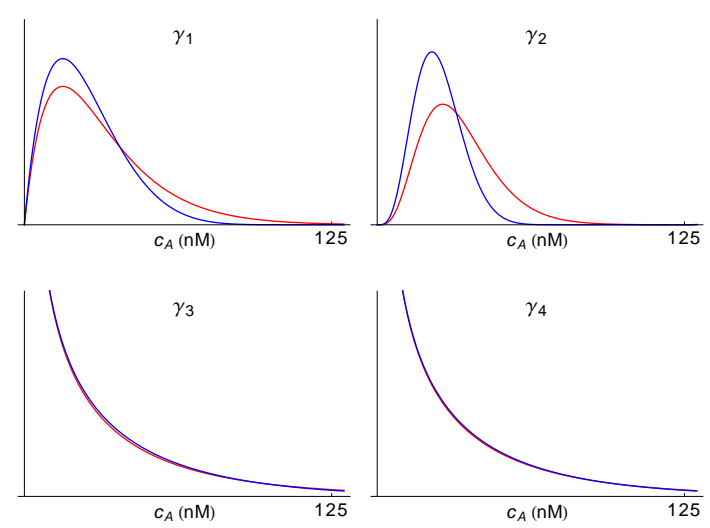

Figure 2.4: Comparison of analytical distributions from protein burst theory and from our modeling approach. Comparison between the gamma distribution (2.22) of the protein burst theory (red line) and the analytical distribution (2.25) derived in our modeling approach when there is no diffusion (blue line), for all parameter sets $\gamma_{1}=(\widetilde{\alpha}, \widetilde{\beta})=(8,2), \gamma_{2}=(15,5), \gamma_{3}=(8,0.5), \gamma_{4}=(15,0.5)$. The analytical distribution based on the dichotomous mRNA dynamics is a good approximation of the protein burst-like production when $\beta \ll \alpha$ as for the parameter sets $\gamma_{3}$ and $\gamma_{4}$.

agreement otherwise. In our modeling approach, the assumption of having at most one mRNA molecule in the cell is needed in order to describe the dynamics of the mRNA by a dichotomous process. This theoretical description allowed us to calculate the analytical distribution of the autoinducer. However, there is no biological reason to assume that once a mRNA is in the cell, no other transcription event could take place. This analysis shows that, despite this nonbiological assumption, the dichotomous process correctly describes the dynamics of the mRNA when the transcription rate is much smaller than the mRNA degradation rate. The resulting distribution is compatible with the distribution obtained in the protein burst expression theory, which makes use of a more realistic description. 


\subsection{RESULTS}

2.4.1 The autoinducer dynamics exhibit different regimes as a function of the diffusion rate

The distribution of $c_{A}$ at the steady-state is computed for the different parameter sets according to the ranges and constraints described above (section 2.3.3). In order to explore the role of the diffusion in the dynamics of the signaling molecule we first study the case $\widetilde{D}=0$. According to the analytical calculations (see inequality (2.20)) in this case two possible distributions for the concentration of $c_{A}$ can be observed depending on the value of $\widetilde{\beta}$. Since $\widetilde{\alpha}>1$ we can expect a maximum only if $\widetilde{\beta}>1$ (note that $k_{-}^{\text {eff }}=1$ if $\widetilde{D}=0$ ), otherwise extrema are not expected. The results of the numerical simulations shown in figure 2.5 $\mathrm{A}$ in fact reveal that scenario. Note that in all cases the histogram obtained from the simulations fits fairly well to the expression (2.15) except for deviations due to the intrinsic noise that are not taken into account by the analytical approach. The differences among dynamics are evidenced by the trajectories (figure 2.5B). Thus, for $\widetilde{\beta}<1$ (parameter sets $\gamma_{3}$ and $\gamma_{4}$ ) the dynamics of the autoinducer shows a burst-like behavior. If $\widetilde{\beta}>1$ (parameter sets $\gamma_{1}$ and $\gamma_{2}$ ) the frequency of bursts is high enough to maintain the concentration of molecules near the average and a single-peak distribution develops.

If $\widetilde{\mathrm{D}}>0$ we expect a more fruitful phenomenology since the transition lines in the parameter space $(\widetilde{\alpha}, \widetilde{\beta})$ shift as a function of the diffusion (see figure 2.2A). According to the analytical calculation we can anticipate that, for a given parameter set and as $\widetilde{D}$ increases, the system explores different dynamical regimes. By taking as a reference the case $\gamma_{2}$, that is $(\widetilde{\alpha}, \widetilde{\beta})=(15,5)$, figure 2.6 shows the effect of the diffusion on the distribution (left column) and dynamics (center column) of $c_{A}$ in a given cell. The system initially displays a singlepeak distribution for $\widetilde{\mathrm{D}}=1$. By increasing the diffusion coefficient we observe transitions to other behaviors (monotonically decreasing and double-peak distributions). The corresponding dynamics of $c_{A}$ (right panels) show how the diffusion, acting as an additional effec- 
A

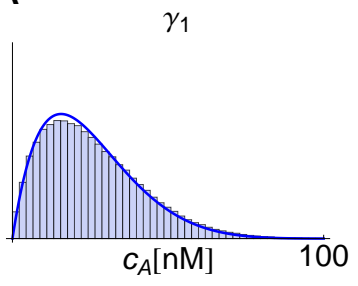

$\gamma_{3}$

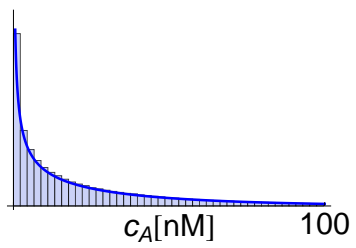

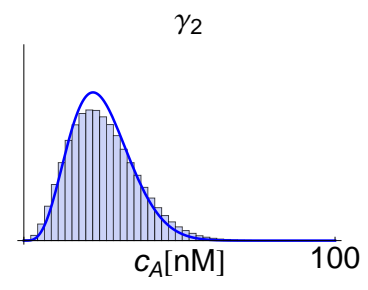

$\gamma_{4}$

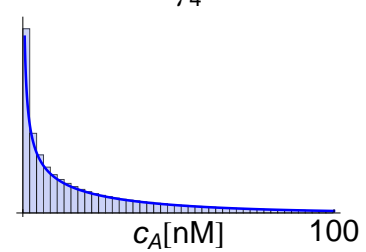

B
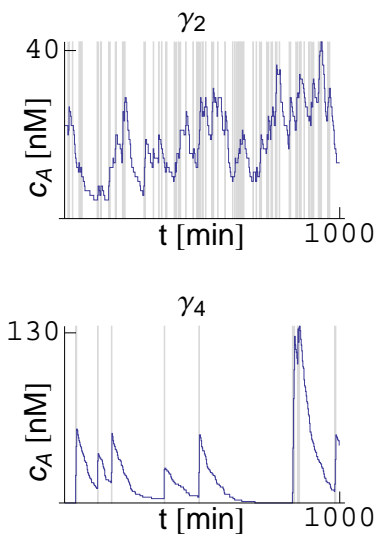

Figure 2.5: Distributions and dynamics of the signaling molecule in a diffusionless system. (A) Distributions of $c_{A}$ at steady-state for different sets of parameters $(\widetilde{\alpha}, \widetilde{\beta})$ as indicated in figure 2.2B. In all cases $\widetilde{\mathrm{D}}=0$. The histogram obtained in simulations (blue bars) compares well with the distribution from the analytical calculations (blue line). Yet, small deviations are observed due to intrinsic noise (see text). (B) The dynamics of the autoinducer show different behaviors depending on the region of the parameters phase space (see figure 2.2). Two typical trajectories are shown with a gray-shaded background indicating the presence of a mRNA molecule in the cell. 
tive degradation on $A$, first increases the sharpness of the bursts of production. For $\widetilde{D}=10$, the diffusion is large enough to remove signaling molecules between consecutive burst events, thus leading to a monotonically decreasing distribution. Increasing the diffusion rate to $\widetilde{D}=100$ leads to the situation where both $\widetilde{\alpha}$ and $\widetilde{\beta}$ becomes smaller than $1+\widetilde{\mathrm{D}}$ and a bistable dynamics develops. Under these circumstances the concentration of autoinducer alternates between two states that correspond to a low concentration when there is no mRNA production and a high concentration following mRNA synthesis. As the diffusion further increases, e.g. $\widetilde{D}=2 \cdot 10^{3}$, the autoinducer molecules diffusing from the external medium into the cell set a constitutive level of this species. The latter explains the presence of A molecules in the cell even if no mRNA is produced. Finally, at very large values of $\widetilde{\mathrm{D}}$, e.g. $\widetilde{\mathrm{D}}=5 \cdot 10^{4}$, the low constitutive concentration of the autoinducer increases due to the influx of molecules when no mRNA is present whereas the concentration of $A$ that is internally produced decreases due to the efflux of molecules. In this case, the whole $\mathrm{N}$-cells system can be considered as a single volume with no diffusive barriers between cells. Thus, the burst events average out and, as a consequence, a single effective peak again develops.

\subsubsection{The intensity of intrinsic fluctuations remains constant when chang- ing the diffusion rate}

Figure 2.6 shows that the theoretical distribution captures the essential features of the dynamics obtained in the numerical simulations (Gillespie). The noticeable deviations are due to the intrinsic noise of the signaling molecule $A$ that are not considered in the theoretical analysis. Notice that as the diffusion increases those deviations seem to be larger. We stress that in our simulations we keep constant the average concentration of the autoinducer by modulating the effective production rate (see Parameters section). As $\widetilde{D}$ becomes larger we increase the production rate $k_{+}$so that the average number of autoinducer molecules per cell remains constant. Consequently, the deviations between the simulations and the theoretical analysis cannot 
be ascribed to a putative decrease of the number of $A$ molecules (i.e. to an increase of the intrinsic noise). Moreover, the deviations cannot be attributed either to a failure of the quasi-steady approximation introduced in equation (2.7) because the larger the diffusion the more accurate that approximation is. As indicated by equation (2.21) the noise due to the mRNA dynamics behaves as $\sim 1 / \widetilde{D}$. Thus, for large values of the diffusion rate, the transcription noise level decreases. Therefore, we must conclude that deviations between the theoretical and the numerical approaches apparently increase with the diffusion rate because there is a drop of the fluctuations related to the mRNA dynamics. This also indicates that for large enough diffusion rate, the intrinsic noise constitutes the main source of stochasticity.

In order to ensure that the intrinsic fluctuations are not actually increasing due to diffusion we first perform the following in silico ex-

Figure 2.6 (following page): Distributions and dynamics of the signaling molecule in a system with diffusion. Distributions (left column) and dynamics (center column) of $c_{A}$ at steady-state for different values of $\widetilde{D}$. In all cases the parameters set $(\widetilde{\alpha}, \widetilde{\beta})$ is $\gamma_{2}$ (see figure $2.2 \mathrm{~B}$ ). The production rate $\tilde{k}_{+}$is modulated as a function of $(\widetilde{\alpha}, \widetilde{\beta}, \widetilde{D})$ in order to maintain constant the average $\left\langle c_{A}\right\rangle=25 \mathrm{nM}$. The histograms obtained in the stochastic simulations (blue bars, left column) are in qualitative agreement with the probability densities from the analytical calculations (blue line, left column). When increasing the diffusion coefficient the system explores different dynamics as also revealed by the trajectories shown in the center column. The gray-shaded background shown in the trajectories of $c_{A}$ indicates the presence of a mRNA molecule in the cell. In order to discern a putative increase in the molecular noise (see section 2.4.2) we perform stochastic simulations of a modified system in which a single mRNA molecule produces two distinguishable autoinducer molecules $A_{1}$ and $A_{2}$. The density plots (right column) of the distribution of $c_{A_{2}}$ vs $c_{A_{1}}$ reveal that the diffusion does not contribute to an increase of the intrinsic noise since the spreading of the distributions in a direction perpendicular to the diagonal does not grow when increasing $\widetilde{\mathrm{D}}$. 

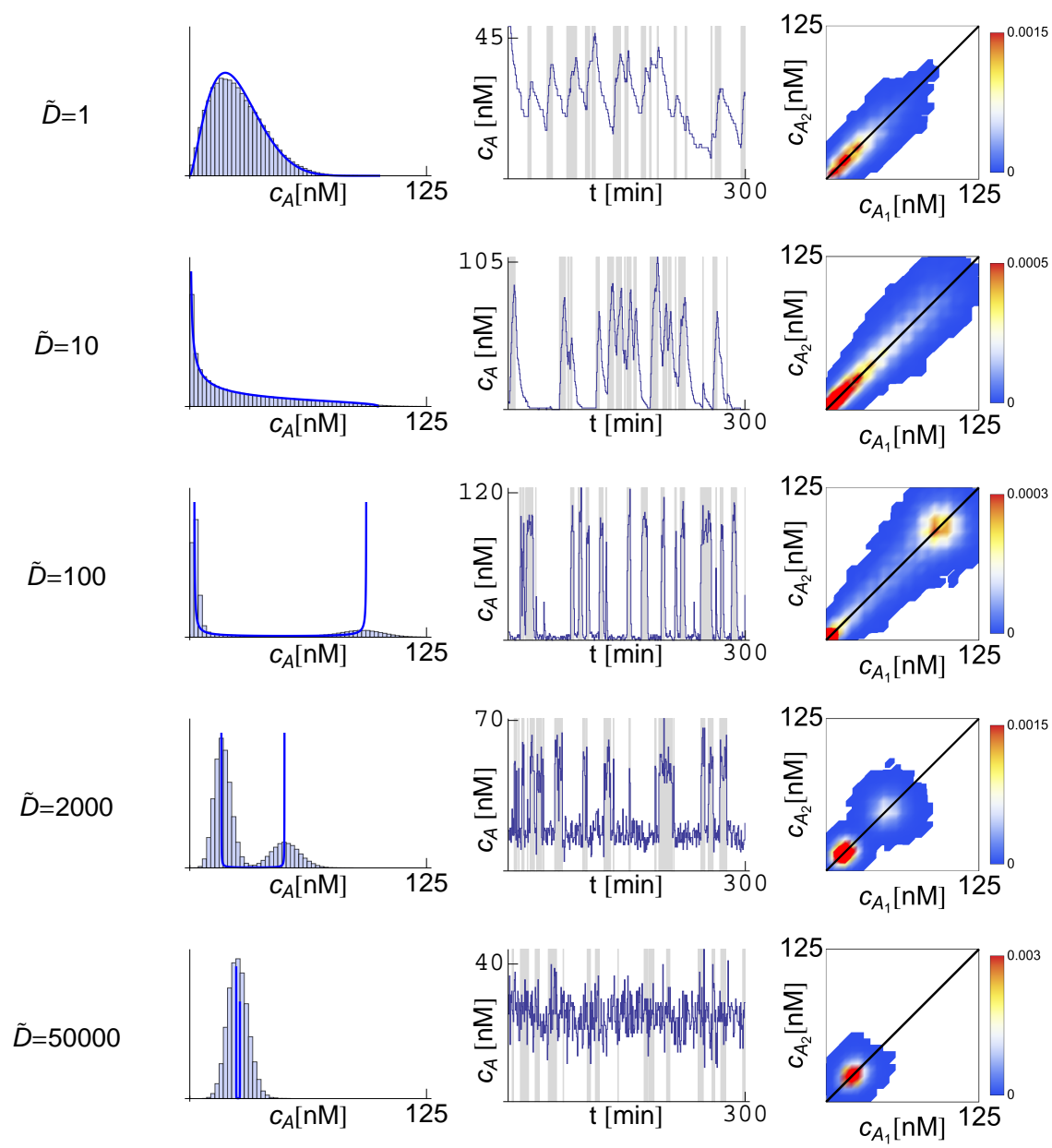
periment. We consider a modification of our system such that a single mRNA molecule transcript leads to two autoinducer molecules that are considered to be distinguishable. As described in section 1.I, the latter can be experimentally achieved by placing consecutively two copies of the encoding sequence of the autoinducer's synthetase in the operon labeled with different fluorescent tags [50]. Thus, we double the set of reactions 2.2 to 2.5 in order to account for $A_{1}^{i}$ and $A_{2}^{i}$ molecules synthesis at cell $i$ due to a single mRNA transcript $M_{1}^{i}$. Following [50], by plotting the distribution of $c_{A_{1}}$ as a function of $c_{A_{2}}$ we can then discern a putative increase of the intrinsic fluctuations (see also section 1.1). Right column of figure 2.6 displays the results in this regard. The width of the distribution in a direction perpendicular to the diagonal is a measure of the intrinsic fluctuations. As shown, as the diffusion increases there is no amplification of this quantity.

\subsubsection{The total noise exhibits a maximum of intensity for intermediate diffusion rates}

It is interesting to place the previous result in the context of the total noise present in the autoinducer concentration. Figure 2.7 reveals that $\eta_{\mathcal{c}_{A}}^{2}$ shows a non-monotonic behavior. As a function of $\widetilde{D}$ the total noise first increases and reaches a maximum at $\widetilde{D} \sim 10^{2}$ and then decreases as the diffusion becomes larger. The same behavior is observed for all four parameter sets. Note that the analytical calculations, that just account for the transcriptional noise, are in agreement with the numerical simulations, that account for both the transcriptional and the intrinsic noise, for a large range of $\widetilde{\mathrm{D}}$ values. This indicates that the main contribution to the total fluctuations for a large range of diffusion values is the transcriptional noise. Yet, as mentioned above, the latter diminishes as the diffusion increases while the intrinsic fluctuations remain constant. Consequently, the contribution of the intrinsic noise must become more relevant than the mRNA dynamics stochasticity beyond some value of $\widetilde{D}$. 
We address this point by calculating the relative importance of the noisy sources. To this end we make use of the decomposition $\eta_{\mathcal{c}_{A}}^{2}=\eta_{\mathcal{c}_{A}, \text { int }}^{2}+\eta_{\mathcal{c}_{A}, \text { tran }}^{2}$, where $\eta_{\mathcal{c}_{A}, \text { int }}^{2}$ and $\eta_{\mathcal{c}_{A}, \text { tran }}^{2}$ stand respectively for the intrinsic and the transcriptional contributions to the total noise [177]. Thus, by subtracting the analytical expression of the transcriptional noise given by equation (2.21) to the total noise obtained in the numerical simulations we are able to compute the intrinsic noise as a function of the diffusion (see figure 2.7). By performing a linear regression of the points that corresponds to the intrinsic noise we obtain that the slope of the curve is indeed zero in practical terms $\left(2 \cdot 10^{-7}\right.$ for parameter set $\left.\gamma_{2}\right)$. Therefore, in agreement with the results obtained in figure 2.6 (right column), the intrinsic noise remains constant $\left(\eta_{\mathcal{C}_{A} \text {,int }}^{2}=0.054 \pm 0.003\right.$ for parameter set $\left.\gamma_{2}\right)$ as the diffusion increases and is the main stochastic component if $\widetilde{\mathrm{D}} \gtrsim 10^{4}$.

\subsection{DISCUSSION}

Most of the stochastic models of gene expression assume that the lifetime of the protein is much longer than the lifetime of the mRNA [166]. This assumption is used, for example, in the protein burst theory to obtain the gamma distribution (2.22). While this is a reasonable assumption for many genes in prokaryotes [178], we have shown that this assumption is not valid for QS systems where the signaling molecule can diffuse. As in pointed in [77], the gamma distribution does not describe accurately the protein distribution when the degradation rate of the protein is similar or smaller to the degradation rate of the mRNA. The degradation rate of the protein is determined by the dilution due to cell growth and by enzymatic degradation. In bacteria that communicate by QS, passive diffusion and active transport through the cell membrane is another mechanism that can greatly modify the effective degradation of the signaling molecule. In this chapter, we have shown how diffusion can dramatically modify the distribution and dynamics of the signaling 

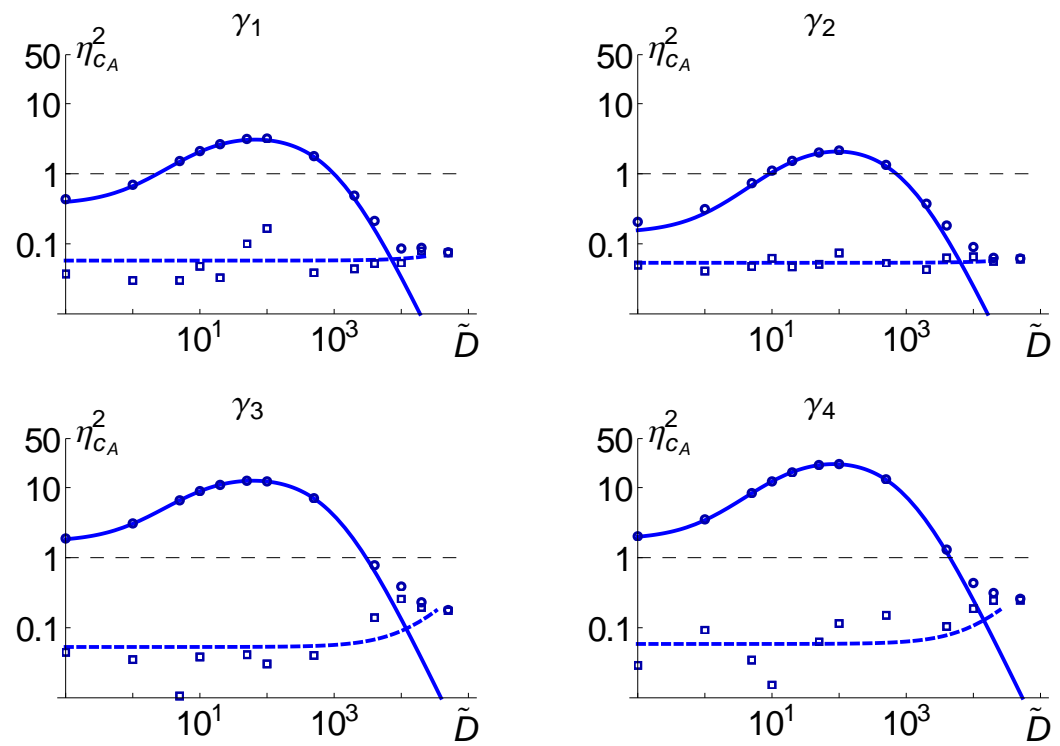

Figure 2.7: Noise in the signaling molecule as a function of the diffusion coefficient. Noise $\eta_{c_{A}}^{2}$ as a function of diffusion coefficient $\widetilde{D}$ for all the sets of parameters $\gamma_{1,2,3,4}$ (see figure 2.2B): stochastic simulations (circles) and analytical expression (2.21) (solid line). By using the decomposition $\eta_{c_{A}}^{2}=\eta_{c_{A} \text {,int }}^{2}+\eta_{c_{A} \text {, ext }}^{2}$, the difference between the spreading of the computational and the theoretical distributions quantifies the amount of intrinsic noise (squares). As evidenced by the linear regression (blue short-dashed line) the latter remains constant and is the main contribution to the total noise only for large diffusion values, $\widetilde{\mathrm{D}}>10^{4}$ (see text). Notice that the linear regression appears as an exponential on the log-log scale because the slope is much smaller than the intercept. The noise function is non-monotonic and has a maximum value above $\eta_{c_{A}}^{2}>1$ for all parameter sets, showing that the variance is larger than the mean for intermediate ranges of $\widetilde{\mathrm{D}}$. 
molecule by modifying the ratio of time scales of the mRNA lifetime and the signaling molecule effective lifetime.

Moreover, we have observed that the non-monotonic behavior of the total noise as a function of the diffusion rate suggests a new interpretation of the role of noise regulation by the QS mechanism. The values of the diffusion rates in QS systems fall into two distinctive categories (see section 2.3.4): either large values corresponding to passive transport mechanism, $\widetilde{\mathrm{D}} \sim 10^{4}$, or small values when an active transport mechanism applies, $\widetilde{\mathrm{D}} \sim 10^{-1}$ to 10 . Surprisingly, these two QS classes avoid diffusion rates that maximize the total noise, $\widetilde{\mathrm{D}} \sim 5 \cdot 10^{1}-10^{2}$. While our modeling is certainly very simple and the derived consequences should be considered with caution, the latter suggests that bacteria have developed mechanisms for coping with the noise and keep their functional QS regime away from the region where $\eta_{c_{A}}^{2}>1$. Notice that the maximum noise in the level of autoinducer is very large and translate into large fluctuations that may perturb the activation of the QS pathway. When looking at the dynamics of the autoinducer for $\widetilde{D}=100$ (see figure 2.6) we observe that the autoinducer jumps between a low state with a few molecules and a high state around $100 \mathrm{nM}$. If the activation threshold lies in between these two values, the QS pathway could get randomly activated due to the fluctuations, even if the average level of autoinducer (always equal to $25 \mathrm{nM}$ in all our simulations) is below the critical concentration. In this regard notice that in every form of information exchange the precision is key. If the precision of the information is fuzzy then the related biological function lacks robustness. Our results point towards the direction that bacteria have adapted their communication mechanisms in order to improve the signal to noise ratio and produce a more reliable information exchange.

Our final comment refers to the possibility of considering other sources of stochasticity. Cell-to-cell variability and extrinsic noise have been proved to act as an important contribution in many cell processes $[50,153,165,32]$. In the context of the problem studied herein, we can envision that variability, either at the level of the mRNA dynamics or at the level of the diffusion rate, can effectively lead to significant changes in the reported phenomenology. How- 
ever, theoretical studies [71, 116] suggest that the quorum sensing synchronization is robust to the variability in the diffusion rate and extracellular noise. In addition, by considering additional steps in the synthesis of the autoinducer, the levels of intrinsic noise could change. Whether or not these additional noise sources, coupled with feedback regulation, may generate new effects in the framework of QS is not known.

\subsection{CONCLUSIONS}

In this chapter I have explored the role played by cell-cell communication and transcriptional noise in QS systems near the activation threshold. We have built a model describing the expression of the signaling molecule and its diffusion. We have focused on the situation where the luxI gene is expressed at a low constitutive level, such that i) the feedback regulation of the luxI gene can be disregarded, and ii) the transcription level is low enough such that at most one mRNA molecule can be present in the cell. Under these conditions the dynamics of the mRNA can be described by a Markovian dichotomous process with random transitions between o and 1 mRNA molecule. Moreover, we assume that the production of the autoinducer can be described by a single reaction step. We have used parameter values that are biologically relevant in the prokaryotes.

We have identified two stochastic contributions that drive the dynamics of the autoinducer: the mRNA fluctuations (transcriptional noise) and the molecular noise (intrinsic noise). We have studied the role played by these two noise sources and their interplay with the diffusion of the autoinducer. First, by neglecting the intrinsic noise, we have derived an analytical expression for the steady-state distribution of the autoinducer concentration. This analytical distribution only takes into account the transcriptional noise. Second, we have computed the steady-state distribution by means of stochastic simulations (Gillespie) that takes into account the transcriptional and the intrinsic noise. By comparing the variability in the autoinducer concentration obtained by the two methods, we have been able to 
elucidate the contribution of each noise source in the total noise of the autoinducer.

We have shown that the dynamics and fluctuations of the signaling molecule exhibits different behaviors depending on the diffusion coefficient. When increasing the rate of diffusion, the probability distribution of the autoinducer changes from single-peak distribution (sustained bursts dynamics), to monotonically decreasing distribution (bursts dynamics), to double-peak distribution (bistable dynamics), and finally to narrow single-peak distribution (diffusionaveraged dynamics).

In addition, we have shown that the interplay between the diffusion and the mRNA dynamics plays a crucial role for regulating the total amount of noise in the number of signaling molecules. Transcriptional noise is the main contribution to the total noise for a large range of diffusion values, $\widetilde{\mathrm{D}}<10^{4}$. Only for very large values of the diffusion the intrinsic noise is the major source of stochasticity. When maintaining the average level of autoinducer, we have shown that the intrinsic noise remains constant when varying $\widetilde{D}$. Importantly, we have shown that the total noise shows a non-monotonic behavior as a function of the diffusion rate. For large values of the diffusion coefficient, the total noise decreases as the diffusion rate increases. In this regard, our results are to be compared to previously reported noise reduction mechanisms, as for example in the case of LuxR/LuxI system [179], or in the case of bistable genetic switches coupled by QS communication [91]. In this regime, diffusion effectively reduces fluctuations by averaging the concentration of the autoinducer between all the cells. On the other hand, when the diffusion rate is comparable to the characteristic rate of mRNA degradation, the total noise increases as the diffusion becomes larger. We have observed that in this regime, the diffusion effectively changes the type of dynamics of the autoinducer. The competition of temporal scales between the lifetime of the mRNA $\left(\widetilde{\alpha}^{-1}\right)$ and the effective degradation of the autoinducer $\left((1+\widetilde{\mathrm{D}})^{-1}\right)$ leads to different dynamical behaviors that exhibit increasing variability.

The regulation of the fluctuations at the level of the autoinducer could have an impact on the biological function of the QS mecha- 
nism. Cell-cell communication can give rise to coordinated behavior at the level of the population, as shown by the density-dependent gene activation observed in QS experiments. However, in every communication system the precision of the information is key. As we have seen, transcriptional and intrinsic noise can give rise to large fluctuations at the level of the signaling molecule. How do these fluctuations interfere with the synchronization of cells during the QS activation? In the next chapter, we will study the role of intra-cellular fluctuations in the activation of the QS pathway when the feeback mechanism of the gene regulatory network and a more realistic cell model are taken into account. 


\subsection{INTRODUCTION}

As first described in the work of Nealson et al. [119], the quorum sensing (QS) phenomenon appeared as a sudden activation of the bioluminescence in a culture of growing Vibrio fischeri cells. By measuring the average light emission and the cell density of a freshly inoculated culture, the experiments suggested a density dependent activation of the luciferase gene. Since then, the QS mechanism has been mainly revealed in different bacterial species at the population level by measuring the average gene expression as a function of cell density.

It was not until recently that the behavior of individual cells has been shown to differ significantly from the bulk behavior, revealing large inter-cell variations in the expression level of QS genes. By immobilizing Vibrio fischeri individual cells, Pérez and Hagen [136] were able to measure the weak bioluminescence of a single bacterium when exposed to a fixed concentration of autoinducer. The authors observed a large cell-to-cell variability in the level of emission and in the onset time for the response. Importantly, this heterogeneity seemed not to be related to the specificities of Vibrio fischeri and was also reported in other QS bacterial species, as Vibrio harveyi [5], Pseudomonas aeruginosa [24], and luxI/luxR-GFP strains of E. coli [65].

As explained in section 1.3, noise may play an important role in the QS activation phenomenon (see also chapter 2). The aforementioned heterogeneity may be caused by the random fluctuations that unavoidably affect cell regulation and signaling. As a matter of fact, a number of studies have shown that noise plays an important role 
in bistable systems $[184,194,52]$ and therefore may influence the activation of the QS bistable switch. This poses the intriguing question of how cells achieve a coordinated response in the presence of noise. Indeed, the QS mechanism may produce a robust and synchronized behavior at the level of the population both experimentally [41] and theoretically [53]. However, how this behavior at the collective level arises from the stochastic dynamics of individual cells is still an open question. At the end, in the framework of QS, a collective response means a precise information exchange in the colony. Consequently, how can a bacterial population estimate its number of constituents precisely if such information is fuzzy at the single cell level? In this chapter, we shed light on this problem and investigate how noise affects the QS transition both at the level of individual cells and at the level of the cell population.

From all the sources of intra-cellular fluctuations, gene expression noise is one of the most important [82]. In the LuxR/LuxI canonical system of Vibrio fischeri (see section 1.2), the two main regulatory proteins LuxR, the receptor of the signaling molecule, and LuxI, the synthetase of the signaling molecule, control the activation of the QS genes. Therefore, fluctuations at the level of the expression of these two proteins can potentially influence the variability in the QS transition. Interestingly, experiments have revealed the presence of additional regulatory interactions for controlling the LuxR noise levels [181], as for example in $V$. harveyi. In this chapter, we study how this variability changes when we modulate the intensity of gene expression noise of LuxR and LuxI. Understanding the role of gene expression noise in this canonical system can help us understand the architecture of more complex QS regulatory networks [121] and whether bacteria have evolved to control the noise and the phenotypic variability.

The LuxR/LuxI system is the simplest QS system and is therefore well suited for mathematical modeling. However, the regulatory interactions that control the wild-type lux operon in Vibrio fischeri are more complex than first thought [109, 163]. Those include both positive and negative regulation of the luxR gene depending on the concentration of the autoinducer [170]. Simplified synthetic constructs 
inserted in E. Coli, such as luxo1 and luxo2 [198], retain the minimal LuxI/LuxR regulatory motif and lack the structural genes responsible for light emission that may also play a regulatory role, e.g. $\operatorname{lu} x D$ [164]. The luxo1 operon is a truncated divergently transcribed lux operon, capable of expressing LuxR but lacking the luxI gene. All the transcripts normally downstream of the promoter are replaced with $g f p$. Bacteria carrying the luxo1 operon cannot produce the autoinducer and therefore addition of exogenous autoinducer is required for GFP expression. On the other hand, the luxo2 operon carries a luxI::gfp fusion and is capable of expressing LuxI and synthesize the autoinducer. These constructs reproduce the main features of the wild-type operon as revealed by the GFP fluorescence reporting the promoter activity [198]. The positive feedback loops mediated by LuxR and LuxI lead to a bistable switch behavior depending on the level of the autoinducer [198, 66]. The decoupling of the two feedback loops in luxo1 offers valuable experimental data on the regulation of LuxR. In this chapter, we focus on the luxo1 and luxo2 constructs as well characterized examples and use the experimental data to fit the parameters in our theoretical approach.

In the context of QS modeling, most research has focused on the understanding of the intracellular circuit $[45,39,62,85,93,198,66$, 179, 125], i.e. single cell studies, while few of them have considered an ensemble of communicating cells [64, 152, 91, 115, 78, 116]. In order to study the effects of intra-cellular fluctuations on the behavior of a population of communicating cells, a multi-cellular description is needed. As part of my thesis, I developed a software (see appendix A.I) capable of simulating a population of cells that can grow and divide, and that includes stochastic simulation of the intra-cellular biochemical reactions and of the diffusion reaction. So far, very few studies [64] have taken into account the coupling of the signaling mechanism at the single cell and collective levels by stochastic means together with realistic dynamics of the proliferation process. 


\subsection{OBJECTIVES AND SUMMARIZED RESULTS}

In this chapter, I present our model for the QS signaling mechanism in Vibrio fischeri based on the synthetic strains luxo1 and luxo2. Our approach takes into account the key regulatory interactions between LuxR and LuxI, the autoinducer transport, the cellular growth and the division dynamics. By using both deterministic and stochastic models, we analyze the response and dynamics at the single-cell level and compare them to the global response at the population level. We aim at studying the effects of gene expression noise on the precision of the QS activation. In a broader context, our study intends to shed light on the relation between the single cell stochastic dynamics and the collective behavior in a population of communicating cells.

Our results indicate that the cell response is highly heterogeneous and that noise in the gene expression of LuxR is the main factor that determines this variability. Moreover, we show that the transition of the QS switch near the critical concentration of autoinducer is very slow compared to the cell cycle duration and that, as a consequence, the non-stationary effects are crucial for setting a precise switch. In addition, we show that increasing the noise in the expression of LuxR helps cells to get activated at lower autoinducer concentrations but, at the same time, slows down the global response. We introduce the concept of precision in order to characterize the reliability of the QS response at the level of the population. The precision is defined as the inverse of the range of autoinducer concentrations for which the cell population response is not fully coordinated (bimodal distribution). We show that the precision of the QS switch under non-stationary conditions decreases with noise, while at steadystate it is independent of the noise value. These results, together with recent experimental evidences on LuxR regulation in wild-type species [181, 189], suggest that bacteria have evolved mechanisms to regulate the intensity of those fluctuations and control the variability in the QS activation.

In the Methods section, I describe our two modeling approaches, deterministic and stochastic, and detail the choice of parameters 
values. In the Results section, I first explain how the deterministic model is used to fit the parameters of the model and reproduce the experimental data at the level of the population average. Then, I present the results of the stochastic model: the cell response distribution in the transient regime and at steady-state, and the statistics of activation time. In the Discussion section I analyze the results both from the theoretical point of view and in the context of the biological function of QS networks. Finally, I summarize our results and discuss some open questions in the Conclusions section.

\subsection{METHODS}

\subsubsection{Modeling of the LuxI/LuxR gene regulatory network}

The luxo1 operon lacks the luxI gene and only $g f p$ is transcribed in that direction. On the other hand, the luxo2 operon carries a luxI::gfp fusion. Accordingly, luxo1 cells cannot produce their own autoinducer and the induction in that case is driven by adding exogenous autoinducer to the medium. Figure 3.1 shows schematically the regulatory interactions we consider in our model. The autoinducer molecules (A) are produced due to the action of their synthetase, LuxI, and bind to the cytoplasmic protein LuxR $(R)$ creating a complex $\left(C_{2}\right)$. The latter binds to the promoter region activating both the transcription of luxI::gfp (only gfp in the case of luxo1) and luxR. Signaling molecules can diffuse passively in and out the cell and contribute to increase the external concentration of the autoinducer $\left(A_{\text {ext }}\right)$ that can be eventually modified by an external influx of molecules $\left(A^{*}\right)$ and a dilution protocol (see section 3.3.4). In our model we consider that signaling molecules degrade at the same rate whether they are cytoplasmic or not. Finally, we consider a DNA duplication process. Such modeling scheme can be formally written as a set of chemical reactions: 


$$
\begin{aligned}
& \text { DNA } \stackrel{\alpha_{R} k_{R}}{\longrightarrow} \quad D N A+m R N A_{l u x R} \\
& \text { DNA } \quad \stackrel{\alpha_{I} k_{I}}{\longrightarrow} \quad D N A+m R N A_{\text {luxI::gfp }} \\
& \mathrm{DNA} \cdot(\operatorname{luxR} \cdot A)_{2} \quad \stackrel{k_{\mathrm{R}}}{\longrightarrow} \quad \mathrm{DNA} \cdot(\operatorname{luxR} \cdot A)_{2}+\mathrm{mRNA}_{\operatorname{luxR}} \\
& \mathrm{DNA} \cdot(\operatorname{luxR} \cdot A)_{2} \quad \stackrel{\mathrm{k}_{\mathrm{I}}}{\longrightarrow} \quad \mathrm{DNA} \cdot(\operatorname{luxR} \cdot A)_{2}+\mathrm{mRNA} \text { luxI::gfp } \\
& m R N A_{l u x R} \stackrel{p_{R}}{\longrightarrow} \quad m R N A_{l u x R}+\operatorname{luxR} \\
& \text { mRNA }_{\text {luxI::gfp }} \stackrel{p_{I}}{\longrightarrow} \quad m_{R N A} \text { luxI::gfp }+ \text { luxI::gfp } \\
& \operatorname{luxI}:: \mathrm{gfp} \quad \stackrel{k_{\mathrm{A}}}{\longrightarrow} \quad A+\operatorname{luxI}:: \mathrm{gfp} \\
& \operatorname{luxR}+A \underset{\mathrm{k}_{1}^{-}}{\stackrel{\mathrm{k}_{1}^{-} / \mathrm{K}_{\mathrm{d} 1}}{\longrightarrow}} \operatorname{luxR} \cdot A \\
& 2(\operatorname{luxR} \cdot A) \underset{\mathrm{k}_{2}^{-}}{\stackrel{\mathrm{k}_{2}^{-} / \mathrm{K}_{\mathrm{d} 2}}{\longrightarrow}}(\operatorname{luxR} \cdot \mathrm{A})_{2} \\
& (\operatorname{luxR} \cdot A)_{2}+\mathrm{DNA} \underset{\mathrm{k}_{\text {lux }}^{-}}{\stackrel{\mathrm{k}_{\text {lux }}^{-} / \mathrm{K}_{\mathrm{dlux}}}{\longrightarrow}} \mathrm{DNA} \cdot(\operatorname{luxR} \cdot A)_{2} \\
& A \stackrel{\mathrm{D}}{\stackrel{\mathrm{rD}}{\longrightarrow}} A_{\text {ext }} \\
& A \stackrel{\mathrm{d}_{\mathrm{A}}}{\rightarrow} \quad \varnothing \\
& A_{\text {ext }} \stackrel{\mathrm{d}_{\mathrm{A}}}{\longrightarrow} \varnothing \\
& \mathrm{mRNA}_{\text {luxR }} \stackrel{\mathrm{d}_{\mathrm{mR}}}{\longrightarrow} \varnothing \\
& m_{R N A} A_{\text {luxI::gfp }} \stackrel{d_{m I}}{\longrightarrow} \varnothing \\
& \operatorname{luxR} \stackrel{\mathrm{d}_{\mathrm{R}}}{\longrightarrow} \quad \varnothing \\
& \operatorname{luxI}:: g f p \stackrel{\mathrm{d}_{\mathrm{I}}}{\longrightarrow} \quad \varnothing \\
& (\operatorname{luxR} \cdot A)_{2} \stackrel{\mathrm{d}_{\mathrm{C}_{2}}}{\longrightarrow} \varnothing \\
& \operatorname{luxR} \cdot A \stackrel{\mathrm{d}_{\mathrm{C}}}{\longrightarrow} \varnothing \\
& \text { DNA } \stackrel{\ln (2) / \tau}{\longrightarrow} \quad \mathrm{DNA}+\mathrm{DNA} \\
& \mathrm{DNA} \cdot(\operatorname{luxR} \cdot A)_{2} \stackrel{\ln (2) / \tau}{\longrightarrow} \mathrm{DNA} \cdot(\operatorname{luxR} \cdot A)_{2}+\mathrm{DNA} .
\end{aligned}
$$




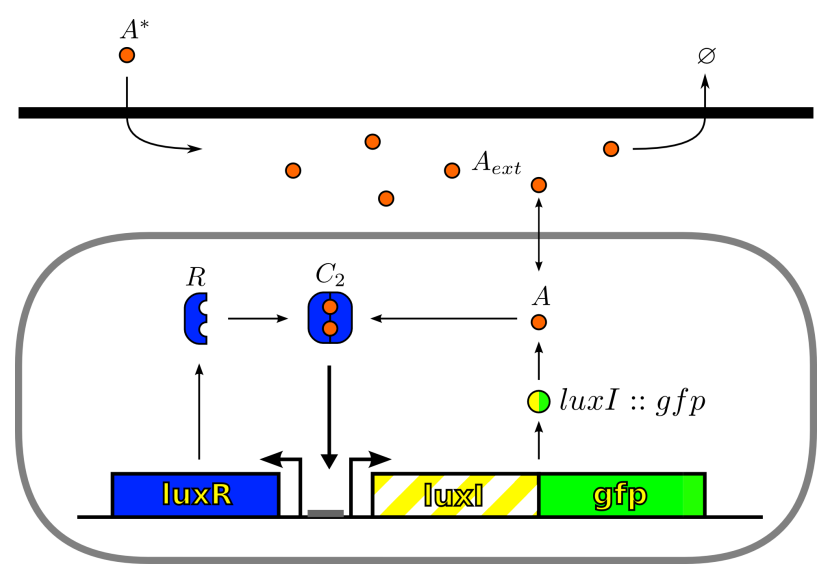

Figure 3.1: Scheme of the LuxI/LuxR regulatory network. The LuxR (R) protein activates the operon upon binding to autoinducer molecules (A). The luxo1 operon lacks the luxI gene and therefore cells cannot produce their own autoinducer and exogenous signaling molecules are needed to activate the expression of $\operatorname{luxR}$ and GFP [198]. On the other hand, the luxO2 operon carries a luxI::gfp fusion and allows for the production of autoinducer and self-induction (see text for details).

As revealed by the set of reactions (3.I), we assume that the regulatory complex (luxR $\cdot A)_{2}$ activates the transcription of $l u x I$ and $\operatorname{luxR}$ in opposite directions upon binding to the DNA. These reactions account for the main regulatory interactions of both $l u x O 1$ and luxo2 constructs. Since luxo1 lacks the luxI gene the autoinducer, $A$, cannot be synthesized, i.e. $k_{A}=0$. The expression rates of $l u x I$ and $\operatorname{luxR}$ depend on the initiation rate of transcription, the speed of elongation, the length of the transcript, and the rate of translation and post-modification into functional proteins. We take into account the differences due to these intermediate processes in an effective manner by using different transcription/translation rates for the luxR and luxI::gfp genes. Note that we assume that there are basal transcriptional rates, $\alpha_{R} k_{R}$ and $\alpha_{I} k_{I}$, even though the regulatory complex $(\operatorname{luxR} \cdot A)_{2}$ is not bound to the promoter region of the DNA 
(the leaky process mentioned in chapter 2). Still, since $\alpha_{R}, \alpha_{I} \ll 1$ (see parameter values in section 3.3.5), the maximum transcriptional rates take place when the activator complex is bound.

\subsubsection{Deterministic and stochastic approaches: cell growth and division}

The reactions (3.1) lead to a Master equation description that can be sampled exactly by means of the Gillespie algorithm [57]. This approach is suitable for the characterization of the system at the single cell level. Complementary to this, if the number of molecules of the species is large enough such that the fluctuations can be neglected, a set of ordinary differential equations (ODEs) can be derived from reactions (3.1) (see equations (3.7) to (3.16)). The ODEs formalism is then appropriate to account for the behavior at the colony level since noise averages out in that case. Notice however that in some cases, the fluctuations can actually modify the behavior of a genetic regulatory network in a more profound way, as will be seen in chapter 4 . Herein we make use of both stochastic and deterministic descriptions as follows. As for the deterministic model, we consider that all cells share their cytoplasm in a single volume $V_{c, \text { tot }}$ (see figure 3.2). Chemical species $X$ inside the cell are described by their concentration, $c_{X}$, in $V_{c, t o t}$. Therefore, this model can only be used to study the dynamics of species averaged over all the cells in the population. From an experimental point of view, the population average could be measured by determining the average bulk fluorescence of the GFP reporter of the cell culture by means of a fluorometer or by averaging the fluorescence data obtained with a flow cytometer.

We notice that our in silico experiments span up to 100 hours of cell culture growth in some cases (simulated experimental time, not computational time). Thus, regardless of the description, and in addition to the dynamics of the regulatory network, we also need to take into account the effects of cell growth. If cells are maintained in the exponential phase with doubling time $\tau$ then the dynamics of the volume of the cell is $V_{c, \text { tot }}(t)=V_{0, t o t} 2^{t / \tau}$. Where $V_{0, \text { tot }}=N V_{0}, N$ being the number of cells in the colony and $V_{0}$ the volume of a single 

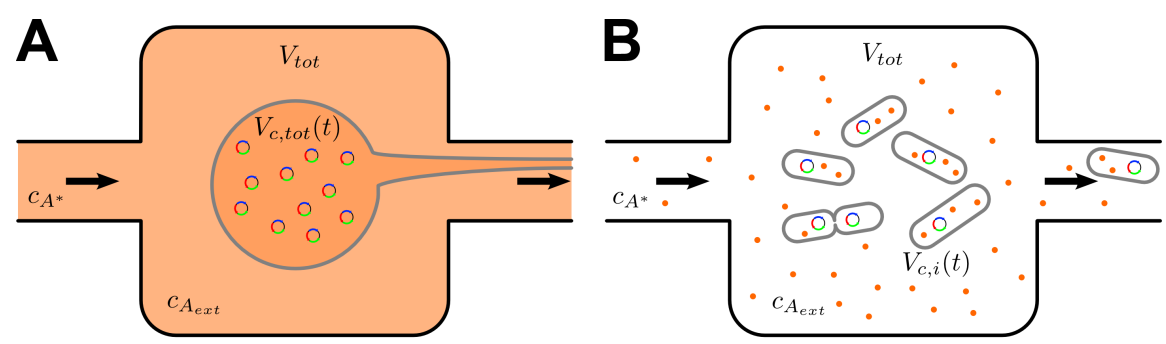

Figure 3.2: Scheme of the deterministic and stochastic modeling approaches. (A) In the deterministic model, the population of cells is described by a unique volume with average and continuous concentrations of all species, including the DNA carrying the QS network (small circles). Cellular growth is also taken into account in this approach. (B) In the stochastic model, cells are modeled as individual compartments that can grow and divide and all molecular species are represented as discrete entities. In both cases, A and B, we assume that all species are well-stirred inside the cells and in the medium. In order to maintain a constant cell density, as in the experiments we aim to model, we implement a dilution protocol. In the deterministic model the dilution removes continuously cytoplasmic material in order to compensate the cell growth. In the stochastic model individual cells are removed every time a new cell is born (see figure 3.5). 
cell at the beginning of the cell cycle. As a consequence, the cellular growth introduces dilution terms, $-c_{X} \frac{\ln (2)}{\tau}$, in the r.h.s. of the ODEs of all species, with the exception of the autoinducer in the medium $A_{\text {ext }}$. On the other hand, cell division events lead to the duplication of the genetic material. The latter is taken into account by adding the term $+\frac{\ln (2)}{\tau}\left(c_{D N A}+c_{\text {DNA. }}(\operatorname{luxR} \cdot A)_{2}\right)$ to the ODE that describes the concentration of DNA. This term compensates exactly for the cell growth dilution such that $c_{D N A, \text { tot }}=c_{D N A}+c_{D N A} \cdot(\text { luxR } \cdot A)_{2}$, i.e. the total concentration of DNA, is kept constant.

In our simulations, as in the experiments we aim to reproduce, the cell density is kept constant. This can be achieved by means of an external dilution protocol (see section 3.3.4) that compensates for cell proliferation. We then keep the volume $V_{c, t o t}$ constant and define the external volume, $V_{\text {ext }}$, such that the total volume of the cell culture reads $V_{\text {tot }}=V_{\text {ext }}+V_{c \text {, tot }}$. Accordingly, the parameter $r$, see reactions (3.I), reads $r=V_{c, \text { tot }} / V_{\text {ext }}$. We assume that molecules are homogeneously distributed inside both the cytoplasm and the external volume (i.e. spatial effects are disregarded). Finally, the resulting ODEs are numerically integrated.

In order to study the role of noise in a population of cells communicating by QS, we have also developed a stochastic model of a population of bacteria. In this case, each bacterium is described as a single cell carrying a copy of the regulatory network. The ensemble of all the chemical reactions in all cells, including the diffusion reaction, are treated as one global system. We apply the Gillespie algorithm [57] to compute the time of the next reaction, choose the reaction channel from the list of all possible reactions and update the number of molecules according to the reaction stoichiometry. We model the system of cells as a global stochastic system in order to simulate as exactly as possible the stochastic dynamics of all chemical species, in particular that of autoinducer molecules. The noise in the signaling molecule originates from different sources: randomness in its synthesis by LuxI, fluctuations at the level of the number of molecules of LuxI, and randomness in the diffusion reaction of the autoinducer. The latter is particularly important since it leads to 
correlations between cells as follows. An autoinducer molecule can diffuse out of the cytoplasm of one cell into the medium, thereby increasing the number of molecules in the external volume by one; this increase in the level of $A_{\text {ext }}$ changes the probabilities of an autoinducer molecule to diffuse into any other cell. Thus, all the cells are coupled through the diffusion reaction. We note that while a possible optimization of the algorithm relies on parallelizing the code such that each cell evolves independently [64], this approximation is prone to introduce errors in the dynamics of the signaling molecule because the aforementioned correlations are neglected.

As mentioned above, cell growth introduces a dilution of the molecules in a cell. We implement cell growth in our stochastic model by allowing the volume of cell $i$ to change in time as,

$$
V_{c, i}(t)=V_{0} 2^{t / \tau_{i}},
$$

where $V_{0}$ is the volume of a cell at the beginning of the cell cycle (same for all cells), $\tau_{i}$ is the duration of the cell cycle of cell $i$, and $t$ is referred to the precedent division event. When $t=\tau_{i}$ the cell $i$ has doubled its volume and a new division takes place. At this time the internal clocks and volumes of daughter cells are reset to zero and $V_{0}$ respectively. Moreover, when a cell divides, proteins, $\mathrm{mR}-$ NAs and signaling molecules are binomially distributed [60, 153] between daughter cells and one copy of the DNA is given to each cell. We note that regulatory complexes bound to the DNA are detached prior to the distribution between daughter cells. As in the case of the deterministic model, we assume that the cell density is maintained constant during experiments due to a compensating external efflux that wash away cells in the culture (see section 3.3.4). In relation to the effect of the cell volume of individual cells on the diffusion rate of the autoinducer, we note that in this case,

$$
r_{i}(t)=\frac{V_{c, i}(t)}{V_{t o t}-\sum_{j=1}^{N} V_{c, j}(t)} .
$$


The duration of the cell cycle, $\tau_{i}$, is different for each cell and is set independently after a division according to the following stochastic rule [32],

$$
\tau_{i}=\lambda \tau+(1-\lambda) \tilde{\tau},
$$

where $\tau$ and $\tilde{\tau}$ denote, respectively, the deterministic and stochastic components of the cell cycle duration, and $\lambda \in[0,1]$ is a parameter that weights their relative importance. The stochastic component accounts for the period of time between events driven by a Poissonian process and satisfies an exponential distribution,

$$
\rho(\tilde{\tau})=\frac{e^{-\frac{\tau}{\tau}}}{\tau} .
$$

In this way, we allow variability from cell to cell in regards of the duration of the cell cycle, yet setting a minimum cell cycle duration, $\lambda \tau$. According to these definitions, the average duration and standard deviation of the cell cycle are $\tau$ and $(1-\lambda) \tau$ respectively.

Finally, we notice that in principle the Gillespie algorithm needs to be adapted in order to take into account the time-dependent cell volume. The propensity of a second-order reaction at cell $i$ at time $t$ scales as $p_{i}(t)=p_{0} V_{0} / V_{c, i}(t)$, where $p_{0}$ stands for propensity of the reaction at division time when $V_{c, i}(0)=V_{0}$. The propensity $p_{0}$ are derived from the corresponding reaction rate, $k$, by dividing the latter by the initial cell volume, $p_{0}=k / v_{0}$. In addition to the change in the propensities of the reaction channels, the algorithm would also need to be adapted to compute the time till next reaction [107]. However, in our case, since all reactions rates are faster than the rate of variation of the cell volume, $\sim 1 / \tau$ (see parameter values in section 3.3.5), then the volume increase is negligible during the time interval until the next reaction takes place. Consequently, we can adiabatically eliminate the volume growth dynamics and safely assume that the volume-dependent propensities remain constant until the next reaction occurs. Summarizing, at a given time $t$ we compute, as described above, the time-dependent propensities based on the volume of the cell at that time and, according to those, 
we determine the time at which the next reaction takes place, $t+\Delta t$, following the standard Gillespie algorithm.

\subsubsection{Gene expression noise: burst size}

During translation mRNA molecules are translated into proteins following a bursting dynamics [82, 202, 30]. The so-called burst size, $b_{X}$, is defined as the ratio between the protein $X$ production rate and the $X$ mRNA degradation rate (see section 1.1 ). It has been shown that $b_{X}$ is directly related to the intensity of gene expression noise $[124,82]$. Thus, for the same average protein concentration, the larger $b_{X}$ is, the more fluctuating expression dynamics is displayed by protein $X$. In our stochastic simulations we use the burst size $b_{X}$ as a parameter to tune the noise intensity at the level of $\operatorname{luxI}$ and $\operatorname{luxR}$ and study its effects. Unless explicitly indicated otherwise, the bursting size in the stochastic simulations is $b_{R}=b_{I}=20[30,181]$.

\subsubsection{External dilution protocol}

In controlled experimental setups it is advantageous to keep the cell density constant. This is carried out by means of an external dilution protocol that compensates for cell growth. Experimentally, this is usually achieved by periodic dilutions of the cell culture [198] or by a continuous flow of liquid medium in a chemostat or in a microfluidic device [19]. This procedure allows to measure the stationary concentration of the signaling molecule at a given cell density and/or to estimate the threshold of the QS collective response of a cell culture. Moreover, the external dilution is also important in order to maintain cells in the exponential growth phase and prevent depletion of nutrients in the medium. Additionally, the levels of the autoinducer can be controlled by adding/removing exogenous signaling molecules in/from the culture buffer. We implement those in our simulations as follows.

In the deterministic model, as shown in figure 3.2, we assume a unique cell with volume $\mathrm{V}_{\mathrm{c} \text {,tot }}$. Cell density is controlled by a con- 
tinuous efflux that removes cytoplasm and culture medium at a rate that compensates exactly for the cell growth, such that the volume $\mathrm{V}_{\mathrm{c} \text {, tot }}$ remains constant. Concurrently, a continuous influx of equal and opposite rate brings fresh medium to the cell culture. In our in silico stochastic experiments, the efflux is reproduced by removing molecules, $A_{\text {ext }}$, from the medium and washing away cells by "deleting" a cell picked at random in the population each time a new cell is born.

In our simulations, the exogenous autoinducer concentration $c_{A^{*}}$ is the control parameter [198]. This means that the levels of autoinducer are controlled by varying the concentration of exogenous autoinducer in the dilution buffer (influx). The influx of exogenous autoinducer molecules, together with the efflux of culture medium, can be represented by the following reaction,

$$
A_{\text {ext }} \underset{\gamma c_{A^{*}} V_{\text {tot }}}{\stackrel{\gamma}{\longrightarrow}} \varnothing \text {. }
$$

where $\gamma=\ln (2) / \tau$. That is, an efflux removes autoinducer molecules from the external volume at a rate $\gamma$ and an influx introduces signaling molecules in the external volume at a rate $\gamma c_{A^{*}} V_{\text {tot }}$. In the deterministic description, this reaction leads to an additional term at the

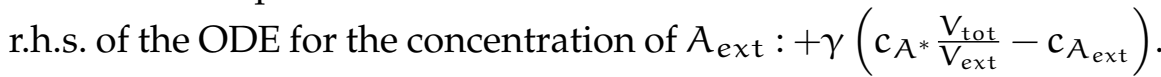
We notice that in our simulations, as in experiments, $V_{\text {tot }} / V_{\text {ext }} \simeq 1$. In the absence of synthesis (e.g. luxo1) and taking into account that the degradation is slower than the diffusion and the influx rate, it is easy to see that the concentration of autoinducer, both inside and outside the cell, tends to $c_{A^{*}}$ : the desired control value of the autoinducer concentration (see figure 3.3).

\subsubsection{Parameters}

The parameters used in our model are listed in table 3.1. When possible, parameter values are fixed or estimated by using experimental measurements found in the literature. The rest of the parameters are fitted to the experimental data of [198] using the deterministic model to reproduce the main characteristics of the response curves of the 
A

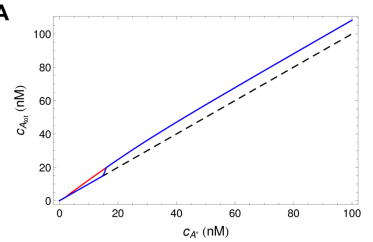

C

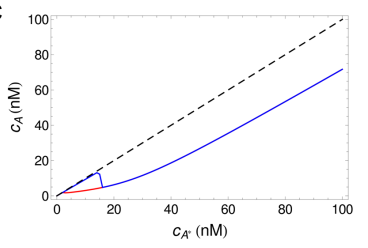

E

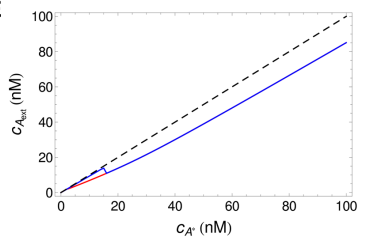

B

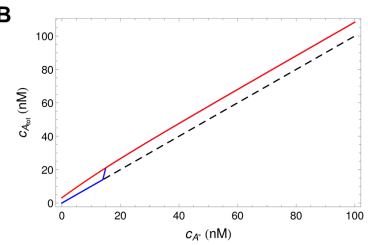

D

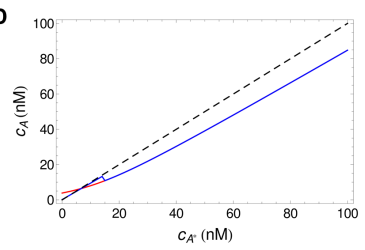

F

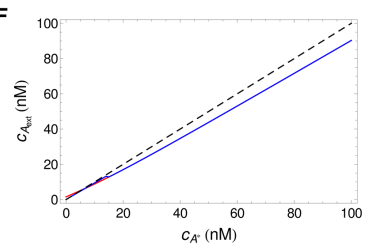

Figure 3.3: Intra and extracellular autoinducer as a function of exogenous autoinducer concentration. Response curves to autoinducer induction for $\operatorname{luxO1}(\mathrm{A}, \mathrm{C}$ and E) and $\operatorname{luxO2}(\mathrm{B}, \mathrm{D}$ and $\mathrm{E}$ ) operons. Total autoinducer concentration $c_{A_{\text {tot }}}$ in the external volume and in the cells (A and $B)$, intracellular concentration $c_{A}(C$ and $D)$, and extracellular concentration $c_{A_{\text {ext }}}(E$ and $F)$, as a function of the exogenous autoinducer concentration, $c_{A^{*}}$, in the deterministic model. All graphs represent the steady-state response for increasing (blue line) and decreasing (red line) autoinducer concentrations. The exogenous autoinducer concentration $c_{A^{*}}$ controls the autoinducer concentration in the medium by means of an influx and an efflux (see section 3.3.4). Upon activation of the operon, LuxR is produced at high levels, thus sequestering autoinducer molecules inside the cells. The bound form of autoinducer cannot diffuse out of the cell and is therefore not subjected to the influx and efflux. This explains why the total concentration of autoinducer in the system, $c_{A_{\text {tot }}}=$ $\frac{1}{V_{\text {tot }}}\left[V_{\text {cell }}\left(c_{A}+c_{\text {luxR.A }}+2 c_{(\operatorname{luxR} \cdot A)_{2}}+2 c_{\text {DNA. }}(\operatorname{luxR} \cdot A)_{2}\right)+\right.$ $\left.V_{\text {ext }} c_{A_{\text {ext }}}\right]$ is slightly larger than $c_{A^{*}}$, when the operon is activated. For the same reason, the concentration of free autoinducer, both in the cell and in the medium, is smaller. 
luxo1 operon: a difference of two orders of magnitude in the level of expression of GFP between the low and the high states, a hysteresis effect in the range of autoinducer concentrations $0 \lesssim c_{A^{*}} \lesssim 15 \mathrm{nM}$, and a time to reach steady-state at full induction $\left(c_{A^{*}}=100 \mathrm{nM}\right)$ shorter than 6 hours. In regards of the cell density, based on an estimate of the CFU/mL for an average OD of 0.5 for E. coli cells, we take a typical value of $c_{N}=5 \cdot 10^{8}$ cells $/ \mathrm{mL}$. Moreover, in order to keep the computational time within reasonable limits, we choose a system size of $N=100$ cells. After fixing the number of cells and the cell density, the total and external volumes are then respectively derived from the relations $\mathrm{c}_{\mathrm{N}}=\mathrm{N} / \mathrm{V}_{\text {tot }}$ and $\mathrm{V}_{\text {ext }}=\mathrm{V}_{\text {tot }}-\mathrm{N} \mathrm{V}_{0}$, where $V_{\text {tot }}=2 \cdot 10^{-4} \mu \mathrm{L}$. Finally, for the case of the luxo2 operon there is one additional parameter that needs to be calibrated: the synthesis rate of the autoinducer, $k_{A}$. The latter is adjusted such that the lower bound of the hysteresis region extends up to $c_{A^{*}}=0$ as experimentally reported.

Table 3.1: Parameters used in the deterministic and stochastic simulations. Abbreviation list: Parameters (Par.), Reference (Ref.), estimated based on experimental values in other biological systems (est.) and fitted to experimental data in [198] (fit.).

\begin{tabular}{|c|c|c|c|}
\hline PAR. & DESCRIPTION & VALUE & REF \\
\hline$K_{\mathrm{d} 1}$ & dissociation constant of LuxR to A & $100 \mathrm{nM}$ & [190] \\
\hline$k_{1}^{-}$ & unbinding rate of LuxR to $A$ & $10 \min ^{-1}$ & est. \\
\hline $\mathrm{K}_{\mathrm{d} 2}$ & $\begin{array}{l}\text { dissociation constant of } \mathrm{LuxR} \cdot \mathrm{AI} \\
\text { dimerization }\end{array}$ & $20 \mathrm{nM}$ & fit. \\
\hline $\mathrm{K}_{2}^{-}$ & $\begin{array}{l}\text { dissociation rate of dimer (LuxR } \\
A I)_{2}\end{array}$ & $1 \min ^{-1}$ & est. \\
\hline$k_{A}$ & synthesis rate of A by LuxI & $0.04 \min ^{-1}$ & fit. \\
\hline$K_{\text {dlux }}$ & $\begin{array}{l}\text { dissociation constant of }(\mathrm{LuxR} \cdot \mathrm{AI})_{2} \\
\text { to the lux promoter }\end{array}$ & $200 \mathrm{nM}$ & fit. \\
\hline$k_{\text {lux }}^{-}$ & $\begin{array}{l}\text { dissociation rate of }(\operatorname{LuxR} \cdot A \mathrm{I})_{2} \text { to } \\
\text { the lux promoter }\end{array}$ & $10 \min ^{-1}$ & est. \\
\hline
\end{tabular}


Table 3.1: (continued)

\begin{tabular}{|c|c|c|c|}
\hline PAR. & DESCRIPTION & VALUE & REF. \\
\hline $\mathrm{b}$ & burst size & 20 & {$[30,181]$} \\
\hline$k_{R}$ & transcription rate of $\operatorname{lux} R$ & $200 / \mathrm{b} \mathrm{min}^{-1}$ & fit. \\
\hline$k_{\mathrm{I}}$ & transcription rate of $l u x I$ & $50 / \mathrm{b} \mathrm{min}^{-1}$ & fit. \\
\hline$p_{R}$ & translation rate of $l u x R$ mRNA & $\mathrm{bd}_{\mathrm{mR}} \min ^{-1}$ & \\
\hline$p_{\text {I }}$ & translation rate of $\operatorname{luxI} \mathrm{mRNA}$ & $\mathrm{bd}_{\mathrm{mI}} \min ^{-1}$ & \\
\hline$\alpha_{R}$ & $\begin{array}{l}\text { ratio between unactivated and acti- } \\
\text { vated rate of expression of } l u x R\end{array}$ & 0.001 & fit. \\
\hline$\alpha_{\mathrm{I}}$ & $\begin{array}{l}\text { ratio between unactivated and acti- } \\
\text { vated rate of expression of } l u x I\end{array}$ & 0.01 & fit. \\
\hline$d_{A}$ & $\begin{array}{l}\text { degradation rate of } A \text { (same inside } \\
\text { and outside the cell) }\end{array}$ & $0.001 \min ^{-1}$ & {$[87]$} \\
\hline $\mathrm{d}_{\mathrm{C}_{2}}$ & degradation rate of $(\mathrm{LuxR} \cdot \mathrm{AI})_{2}$ & $0.002 \min ^{-1}$ & est. \\
\hline $\mathrm{d}_{\mathrm{C}}$ & degradation rate of $\operatorname{LuxR} \cdot \mathrm{AI}$ & $0.002 \min ^{-1}$ & est. \\
\hline$d_{R}$ & degradation rate of LuxR & $0.002 \min ^{-1}$ & est. \\
\hline $\mathrm{d}_{\mathrm{I}}$ & degradation rate of LuxI & $0.01 \min ^{-1}$ & est. \\
\hline$d_{m R}$ & degradation rate of $l u x R$ mRNA & $0.347 \min ^{-1}$ & {$[150]$} \\
\hline $\mathrm{d}_{\mathrm{mI}}$ & degradation rate of $l u x I$ mRNA & $0.347 \min ^{-1}$ & {$[150]$} \\
\hline $\mathrm{D}$ & $\begin{array}{l}\text { effective diffusion rate of } A \text { through } \\
\text { the cell membrane }\end{array}$ & $10 \min ^{-1}$ & {$[84]$} \\
\hline$\tau$ & $\begin{array}{l}\text { cell cycle duration (doubling time) in } \\
\mathrm{RM} \text { / succinate at } 30 \mathrm{C}\end{array}$ & $45 \min$ & [198] \\
\hline$\lambda$ & $\begin{array}{l}\text { relative weight between the det./sto. } \\
\text { components of the cell cycle }\end{array}$ & 0.8 & {$[153,147]$} \\
\hline$v_{0}$ & $\begin{array}{l}\text { cell volume at the beginning of cell } \\
\text { cycle }\end{array}$ & $1.5 \mu \mathrm{m}^{3}$ & {$[187]$} \\
\hline
\end{tabular}


Table 3.1: (continued)

\begin{tabular}{lccc}
\hline PAR. & DESCRIPTION & VALUE & REF. \\
\hline$V_{\text {tot }}$ & total cell culture volume & $2 \cdot 10^{-4} \mu l$ & \\
\hline
\end{tabular}

\subsubsection{First passage time analysis}

The mean first passage time at a given autoinducer concentration quantifies the average time that a cell takes to get activated or deactivated. For computing the first passage time in transitions, from low (high) to high (low) state, we take a single cell at the low (high) state and follow its dynamics until the GFP expression level reaches the high (low) state. We point out that the maximum GFP concentration refers to that of the deterministic simulations. In order to get enough statistics, we repeat this procedure, departing from the same initial condition, $10^{3}$ times for each concentration of autoinducer.

\subsection{RESULTS}

3.4.1 The deterministic model reproduces the experimental observations at the population level

The chemical kinetics formalism leads to a set of ODEs that describes the population average dynamics in terms of the concentration of the different species considered in our model:

$$
\begin{array}{r}
\dot{c}_{\text {luxI::gfp }}=p_{I} c_{m R N A_{\text {luxI::gfp }}}-\left(\frac{\ln (2)}{\tau}+d_{I}\right) c_{\text {luxI::gfp }} \\
\dot{c}_{\text {luxR }}=-\frac{k_{1}^{-}}{k_{d} 1} c_{A} c_{\text {luxR }}+k_{1}^{-} c_{\text {luxR }} A+p_{R} c_{m R N A} A_{\text {luxR }} \\
-\left(\frac{\ln (2)}{\tau}+d_{R}\right) c_{\text {luxR }}
\end{array}
$$




$$
\begin{aligned}
\dot{c}_{m R N A_{\text {luxI::gfp }}=\alpha_{I} k_{I} c_{D N A}}+k_{I} c_{D N A} \cdot(\operatorname{luxR} \cdot A)_{2} \\
-\left(\frac{\ln (2)}{\tau}+d_{m I}\right) c_{m R N A_{\text {luxI::gfp }}}
\end{aligned}
$$

$$
\begin{aligned}
& \dot{\mathrm{c}}_{\mathrm{mRNA}} \mathrm{luxR}_{\text {lut }}=\alpha_{\mathrm{R}} \mathrm{k}_{\mathrm{R}} \mathrm{c}_{\mathrm{DNA}}+\mathrm{k}_{\mathrm{R}} \mathrm{c}_{\mathrm{DNA}} \cdot(\text { luxR } \cdot \mathrm{A})_{2} \\
& -\left(\frac{\ln (2)}{\tau}+d_{m R}\right) c_{m R N A_{\text {lux }}}
\end{aligned}
$$

$$
\begin{aligned}
\dot{c}_{\operatorname{luxR} \cdot A}=- & k_{1}^{-} c_{\operatorname{luxR} \cdot A}+\frac{k_{1}^{-}}{k_{d 1}} c_{A} c_{\operatorname{luxR}}-2 \frac{k_{2}^{-}}{K_{d 2}}\left[c_{\operatorname{luxR} \cdot A}\right]^{2} \\
& +2 k_{2}^{-} c_{(\operatorname{luxR} \cdot A)_{2}}-\left(\frac{\ln (2)}{\tau}+d_{C}\right) c_{\text {luxR } \cdot A}
\end{aligned}
$$

$$
\begin{aligned}
& \dot{\mathrm{c}}_{(\operatorname{luxR} \cdot \mathrm{A})_{2}}=\frac{\mathrm{k}_{2}^{-}}{\mathrm{K}_{\mathrm{d} 2}}\left[\mathrm{c}_{\operatorname{luxR} \cdot \mathrm{A}}\right]^{2}-\mathrm{k}_{2}^{-} \mathrm{c}_{(\operatorname{lu} \times R \cdot A)_{2}} \\
& -\frac{k_{l u x}^{-}}{K_{\text {dlux }}} c_{(\operatorname{luxR} \cdot A)_{2}} c_{D N A}+k_{l u x}^{-} c_{D N A}(\operatorname{luxR} \cdot A)_{2} \\
& -\left(\frac{\ln (2)}{\tau}+d_{C_{2}}\right) c_{(\operatorname{luxR} \cdot A)_{2}}
\end{aligned}
$$

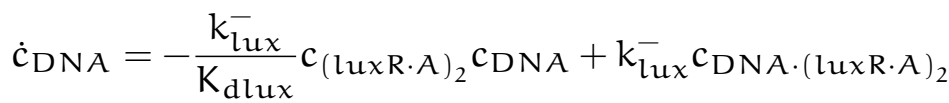

$$
\begin{aligned}
& +\frac{\ln (2)}{\tau}\left(c_{\text {DNA }}+c_{\text {DNA } \cdot(\operatorname{luxR} \cdot A)_{2}}\right)-\frac{\ln (2)}{\tau} c_{D N A}
\end{aligned}
$$

$$
\begin{aligned}
& \dot{\mathrm{c}}_{\mathrm{DNA} \cdot(\operatorname{luxR} \cdot \mathrm{A})_{2}}=\frac{\mathrm{k}_{\mathrm{lux}}^{-}}{\mathrm{K}_{\mathrm{dlux}}} \mathrm{c}_{(\operatorname{luxR} \cdot \mathrm{A})_{2}} \mathrm{c}_{\mathrm{DNA}} \\
& -k_{\operatorname{lux}}^{-} c_{D N A} \cdot(\operatorname{luxR} \cdot A)_{2}-\frac{\ln (2)}{\tau} c_{D N A \cdot(\operatorname{luxR} \cdot A)_{2}}
\end{aligned}
$$

$$
\begin{aligned}
\dot{c}_{A}=k_{1}^{-} c_{\text {luxR } \cdot A} & -\frac{k_{1}^{-}}{K_{d 1}} c_{A} c_{l u x R}+k_{A} c_{l u x I:: g f p} \\
& +D\left(c_{A_{\text {ext }}}-c_{A}\right)-\left(\frac{\ln (2)}{\tau}+d_{A}\right) c_{A}
\end{aligned}
$$




$$
\dot{c}_{A_{e x t}}=r D\left(c_{A}-c_{A_{e x t}}\right)+\gamma \frac{V_{t o t}}{V_{e x t}} c_{A^{*}}-\left(\gamma+d_{A}\right) c_{A_{e x t}}
$$

As explained in the section 3.3.4, we assume that the cell culture grows in an environment where the concentration of the external autoinducer in the medium, $c_{A_{\text {ext }}}$, is kept fixed and under well-stirred conditions. In addition, we implement a dilution protocol that compensates for cell growth and maintains the cell density constant.

We use the deterministic simulations as a benchmark of the regulatory interactions included in our model and also to fit/estimate some parameters such that the experimental data are reproduced (see [198]). Thus, by integrating numerically the rate equations (3.7) to (3.16) derived from the population-averaged model, we compute the steady state concentration (induction time 100 hours) of GFP (luxO1) and LuxI::GFP (luxO2) as a function of $\mathrm{c}_{\mathrm{A}^{*}}$. The steady-state induction curves for increasing and decreasing autoinducer concentration of the luxO1 and luxO2 constructs are shown in figure 3.4. We are able to reproduce the behavior of the network at the steady-state, in particular a region of bistability in the range of autoinducer concentration $2 \mathrm{nM}<\mathrm{c}_{\mathrm{A}^{*}}<15 \mathrm{nM}$ (luxO1) and $0 \mathrm{nM}<\mathrm{c}_{\mathrm{A}^{*}}<15 \mathrm{nM}$ (lux02). As shown by Williams et al. [198], the $l u x R$ regulation of the luxo1 operon alone (positive feedback loop) is enough to yield a bistable response. Moreover, expression of LuxI in the luxo2 operon restores the autoinduction loop and extends the lower bound of the bistable range to zero concentration of exogenous autoinducer as seen experimentally, indicating that once the operon is fully activated and cells produce their own autoinducer that increases the stability of the high state.

Further simulations to check if the dynamics of our model is compatible with the experimental data refer to the behavior of the system under non-stationary induction conditions and to the serial dilution protocol of the external medium [198]. As for the first, when cells are induced for 1o hours, we observe that the bistability region increases (see figure 3.4). As for the second, cells are partially induced at a fixed autoinducer concentration for 2 hours and afterwards the external medium is changed hourly to decrease the concentration of the autoinducer. In this case, the transient response of the cells (fig- 
A

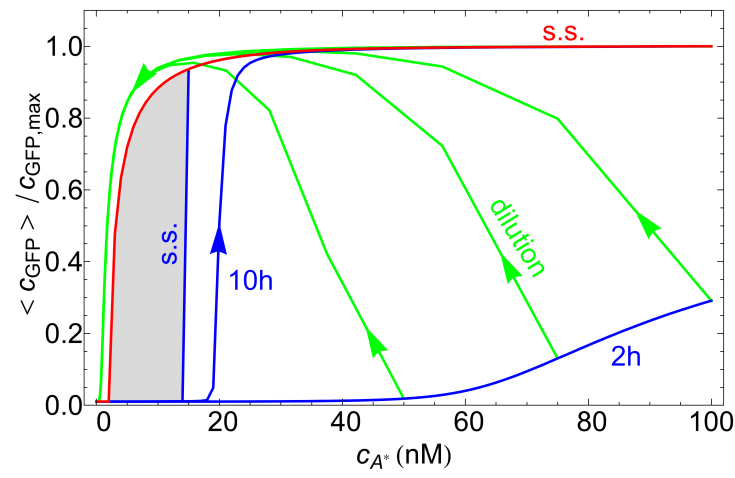

B

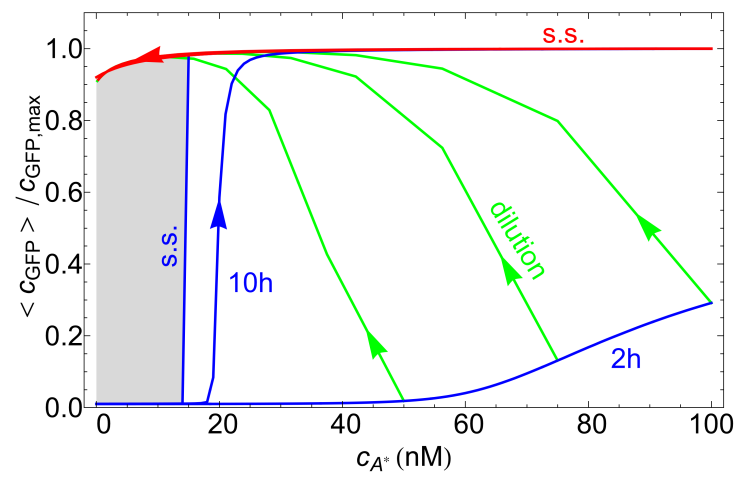

Figure 3.4: Response curves to autoinducer induction in the population-average model. luxo1 (A) and luxo2 (B) operons. The normalized GFP concentration is plotted as a function of the exogenous autoinducer concentration $c_{A^{*}}$ : steady-state (s.s.) response for increasing (arrow-free upper blue curve) and decreasing (arrow-free red curve) autoinducer concentration, response under $10 \mathrm{~h}$ induction time for increasing (blue curve with arrow, 1oh) autoinducer concentration, transient response after 2 hours of induction (lower blue curve, 2h) from initially non-induced cells, decreasing-concentration trajectories (green curves, dilution) for cells weakly induced for 2 hours at $c_{A^{*}}=100 \mathrm{nM}$, $75 \mathrm{nM}$ and $50 \mathrm{nM}$, and decreasing-concentration trajectories (red curve with arrow) for cells fully induced (10 hours) at $\mathrm{c}_{A^{*}}=100 \mathrm{nM}$. The decreasing-concentration trajectories reduce the value of $c_{A^{*}}$ hourly by $25 \%$ (similar to the experiments in [198]). The gray-shaded region between the increasing and decreasing steady-state curves reveals bistability in the range $2 \mathrm{nM}<\mathrm{c}_{A^{*}}<15 \mathrm{nM}$ (luxo1) and $0 \mathrm{nM}<\mathrm{c}_{A^{*}}<15 \mathrm{nM}$ (luxo2). 

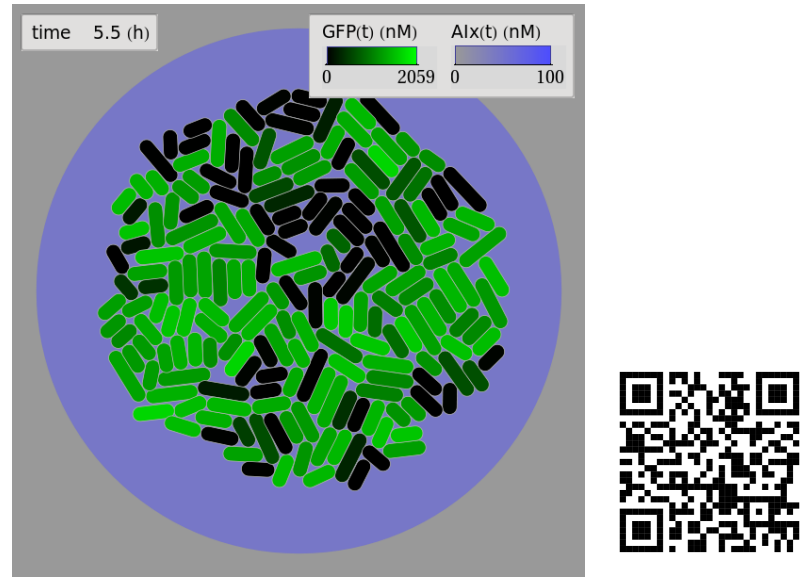

http: / / tinyurl.com/lgtwx5y

Figure 3.5: Movie of the stochastic simulation. Movie of the stochastic simulation using our Colony simulation software (see appendix A.1) for the luxO2 operon, $10 \mathrm{~h}$ of induction at $\mathrm{c}_{\mathrm{A}^{*}}=50 \mathrm{nM}$, burst size $b_{R}=b_{I}=4$. Cells are modeled as individual compartments containing a copy of the LuxR/LuxI regulatory network. The Gillespie algorithm [57] is used to integrate the stochastic dynamics of the whole system of cells. Cell growth and division is explicitly taken into account as well as a certain degree of stochasticity in the cell cycle duration. Cells movement is purely aesthetic since we do not include any spatial effects in our model and consider a well-mixed environment. The number of cells $(N=100)$ is maintained constant by removing one cell at random each time a cell divides. 
ure 3.4, green curves) also reproduces qualitatively the experimental observations. That is, from the point of view of the population average, the deterministic model is not only capable of reproducing the steady-state of the network but also its non-stationary dynamics. Moreover, in agreement with experiments (see figure S6 in [198]) our simulations reveal that the temporal scale for reaching a steady-state is much larger than the cell cycle duration. In order to clarify how noise and the induction time modifies the timing for the transition at the single cell level we then perform stochastic simulations.

\subsubsection{The stochastic simulations reveal the interplay between non-statio- nary effects and noise}

Cells are subjected to intrinsic noise at the level of the mRNAs, regulatory proteins, i.e. LuxR and LuxI, and at the level of signaling molecules. In order to analyze the behavior of individual cells and reveal how noise affects the QS switch, we perform stochastic simulations of a population of growing and dividing cells as described in the Methods section (see also figure 3.5). The transition of an individual cell from the low to the high state, and the other way around, is intrinsically random and depends, among others, on the levels of autoinducer. Thus, inside a population some cells will jump while others remain in their current state leading to a bimodal phenotypic distribution. We compute the proportion of cells that are below and above a threshold of GFP equal to half-maximum GFP concentration. We consider the distribution of cells to be bimodal when the proportion of cells in either the low or the high state is below $90 \%$ and according to this we define the range of autoinducer concentration $\left[c_{A_{b 1}^{*}}, c_{A_{b 2}^{*}}\right]$ for which there is bimodality. For low concentrations of autoinducer, $c_{A^{*}}<c_{A_{b 1}^{*}}$, the collective response of the cell population is unactivated, and for high concentrations, $c_{A^{*}}>c_{A_{b 2}^{*}}$, such response activates most of the cells leading to a global response of the colony. On the other hand, within the bimodality range, the response is distributed between two subpopulations, thus failing to achieve a global coordination in the colony. In order to characterize 
this behavior, we introduce the concept of precision in the QS switch as the inverse of the $c_{A_{*}}$ concentration range for which the cells response distribution (phenotypes), during an induction experiment, is bimodal. That is, the larger the bimodal range, the less precise the switch is in order to generate a global response in the colony. In this regard, we point out that the precision of the switch in a noise-free situation is infinite since all cells achieve global coordination simultaneously.

Figure 3.6 shows, by means of a color density plot, the probability of a cell to have a particular GFP expression level after either 10 or 100 hours of induction as a function of $c_{A^{*}}$. In order to gather enough statistics, we average our results over 10 different realiza-

Figure 3.6 (following page): Cell response distribution to autoinducer induction in the stochastic model. Cell response probability after 1o hours (top: A, B) and 100 hours (middle: C, D) of induction at different autoinducer concentrations $c_{A^{*}}$ for the luxo1 (left: $A, C$ ) and lux02 (right: B, D) operons in the stochastic model. The distribution reveals the coexistence of two subpopulations with low and high GFP expression when the cells are induced at intermediate autoinducer concentrations. The region of bistability (inverse of the precision) is defined by the range of $c_{A^{*}}$ for which the response is bimodal according to the following criterion: the lower/upper limit of the bistable region (orange lines) is defined by the value of $c_{A^{*}}$ for which $90 \%$ of the cells are in the low/high state. The black line stands for the concentration of GFP (normalized) as a function of $c_{A^{*}}$ in the deterministic model at the steady state. After to hours of induction (top: A, B) most cells are still in a transient state if $c_{A^{*}}<70 \mathrm{nM}$. After 100 hours of induction (middle: $\mathrm{C}, \mathrm{D}$ ), the bimodality region shrinks and the precision increases. The population average curves of the steady-state induction and dilution experiments in the stochastic model (bottom: E, F, dashed lines) show that the intrinsic noise allows cells to jump to the high state inside the deterministic bistable region. On the other hand, the transition from high to low follows the deterministic path thus indicating that the switching rate in this case is close to zero. 

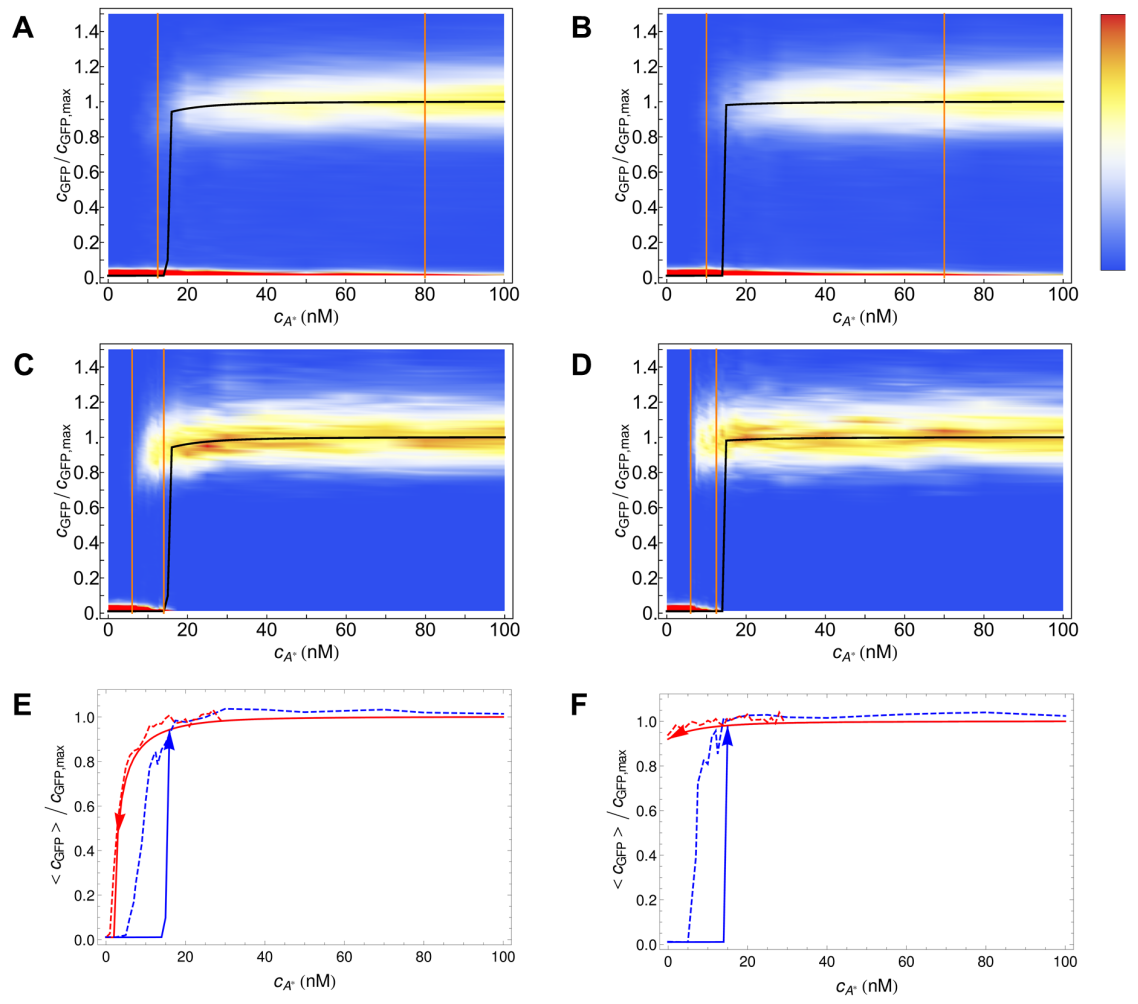
tions (i.e. experiments). For a large range of autoinducer concentrations, for both the luxO1 and for the luxO2 operon, the distribution of GFP expression after $10 \mathrm{~h}$ of induction is bimodal. As shown, some cells of the colony are induced before the critical concentration of the deterministic model at the steady state (black line). Still, the concentration for which more than $90 \%$ of the cells are induced requires up to four times more autoinducer than under deterministic conditions. Thus, on the one hand noise can help cells to get induced at lower autoinducer concentrations but, on the other hand, amplifies the non-stationary effects for achieving global coordination. In order to clarify this interplay between non-stationary and stochastic effects, we perform the same simulations with a larger induction time $(100 \mathrm{~h})$. As expected, the precision of the switch increases (10-fold change) and cells achieve global coordination at (luxo1) or before (luxO2) the critical deterministic concentration. Note that in all cases noise induces a significant variability in terms of the GFP expression levels in the high state compared to that of the low state (see also figure 3.7). The variability introduced in the colony response by the fluctuations with respect to the deterministic approach can also be observed in experiments under weak inducing conditions where the autoinducer concentration is periodically decreased (see figure 3.8).

The heterogeneity in terms of the jumping statistics is revealed in figure 3.7 where we plot individual trajectories for the luxO1 operon as a function of time at $c_{A^{*}}=25 \mathrm{nM}$ over a period of 50 hours. Some cells become induced after 3 hours, while others need $\sim 10$ times more induction time to reach the high state. At this concentration of autoinducer all cells have eventually reached the high state after $\sim 30$ hours of induction. Importantly, we do not observe that cells jump back (see section 3.5). That is, while there is variability over the colony in regards of the switching time, once the transition occurs the cell remains in the new state that is sustained over generations as seen in figure 3.9. Therefore, over the typical timescale of an experiment (10 to 50 hours), the behavior of the QS switch is highly dynamic and the precision of the switch is a transient quantity that crucially depends on the duration of induction. 


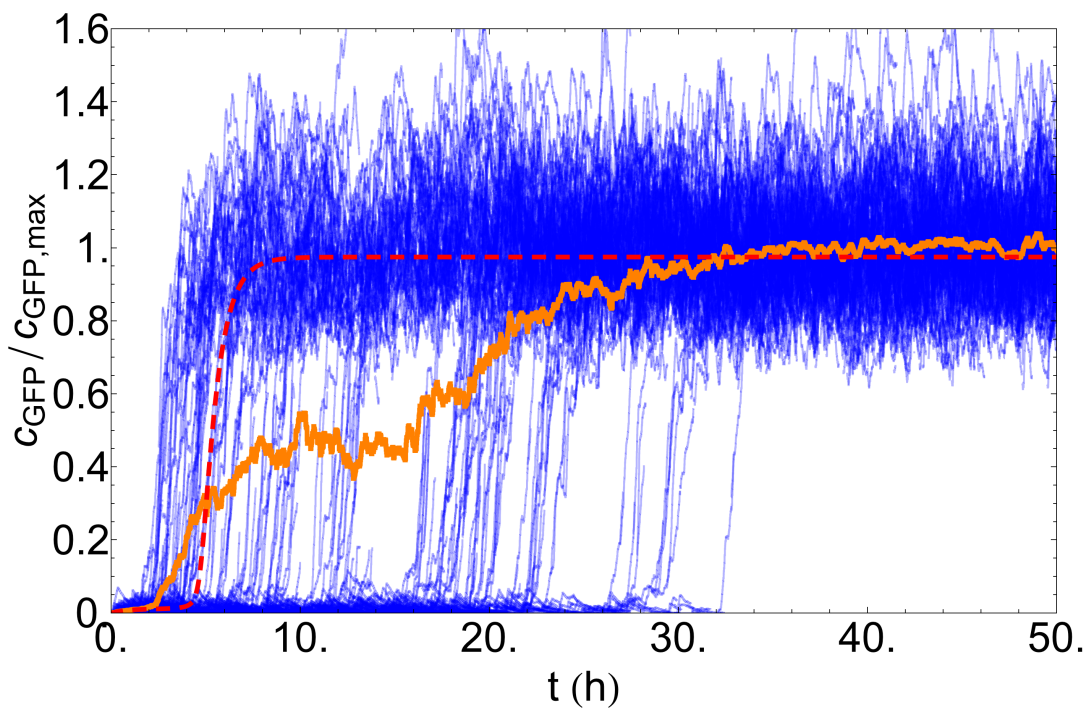

Figure 3.7: Individual cell trajectories for autoinducer induction in the stochastic model. Individual cell trajectories (blue lines) and cell population average (orange line) for an induction experiment at $\mathrm{c}_{\mathrm{A}^{*}}=25 \mathrm{nM}$ for the luxo1 operon in the stochastic model. Individual cell trajectories show the heterogeneous distribution of cell jumping times. While some cells achieve full induction of the operon before the deterministic solution (red dashed line), the global response of the population reaches steady-state at $\sim 30$ hours, slower than the in the deterministic case. 

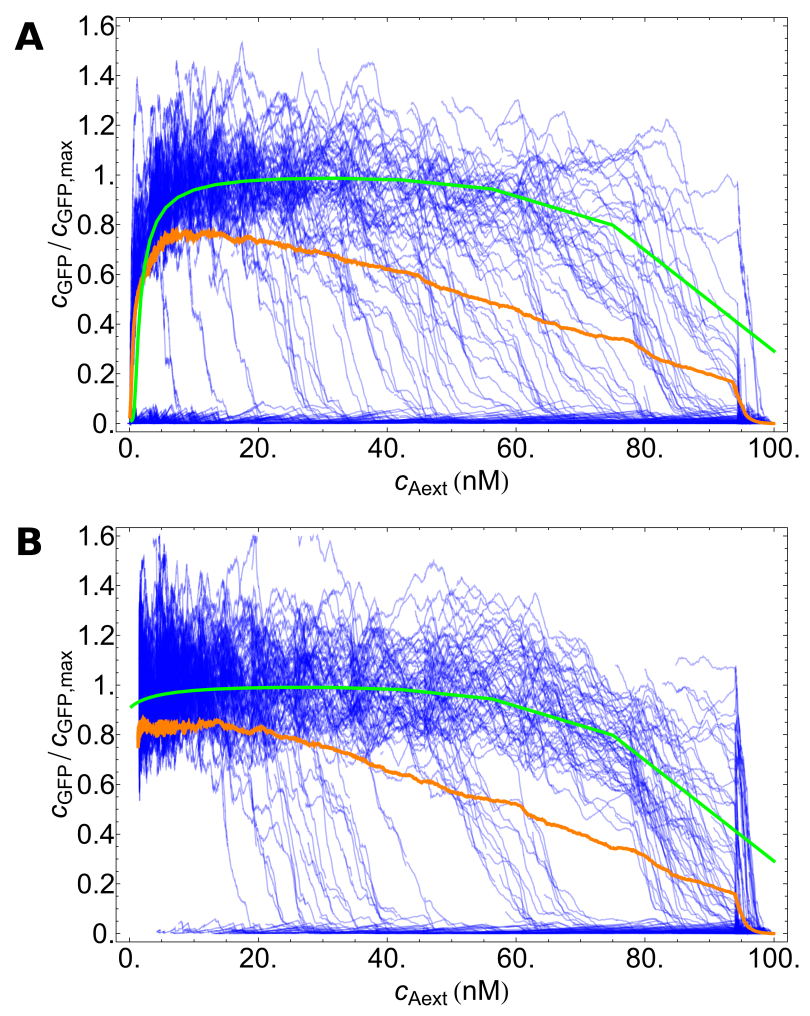

Figure 3.8: Individual cell trajectories during decreasing autoinducer concentration experiment in the stochastic model. Individual cell trajectories (blue lines), cell population average (orange line) and deterministic solution (green line) for dilution experiment plotted as GFP normalized expression vs. external autoinducer concentration $c_{A_{\text {ext }}}$. Cells are initially induced at $c_{A^{*}}=100 \mathrm{nM}$ for 2 hours. The concentration of exogenous autoinducer $c_{A *}$ is then hourly decreased by $25 \%$ in order to simulate the experiments (see [198]), leading to a similar decrease in $c_{A_{\text {ext }}}$. The individual cell trajectories reveal the heterogeneity in the cell activation and deactivation times. For the luxo1 operon (A), cells eventually go back to the unactivated state when reaching zero autoinducer concentration, while cells carrying the luxo2 operon (B) remain in the activated state. Cells response is clearly bistable, with some of the cells staying unactivated for all autoinducer concentrations while other cells jump to the fully active state. The bimodality due to stochasticity cannot be detected at the level of population average or in the deterministic model. 


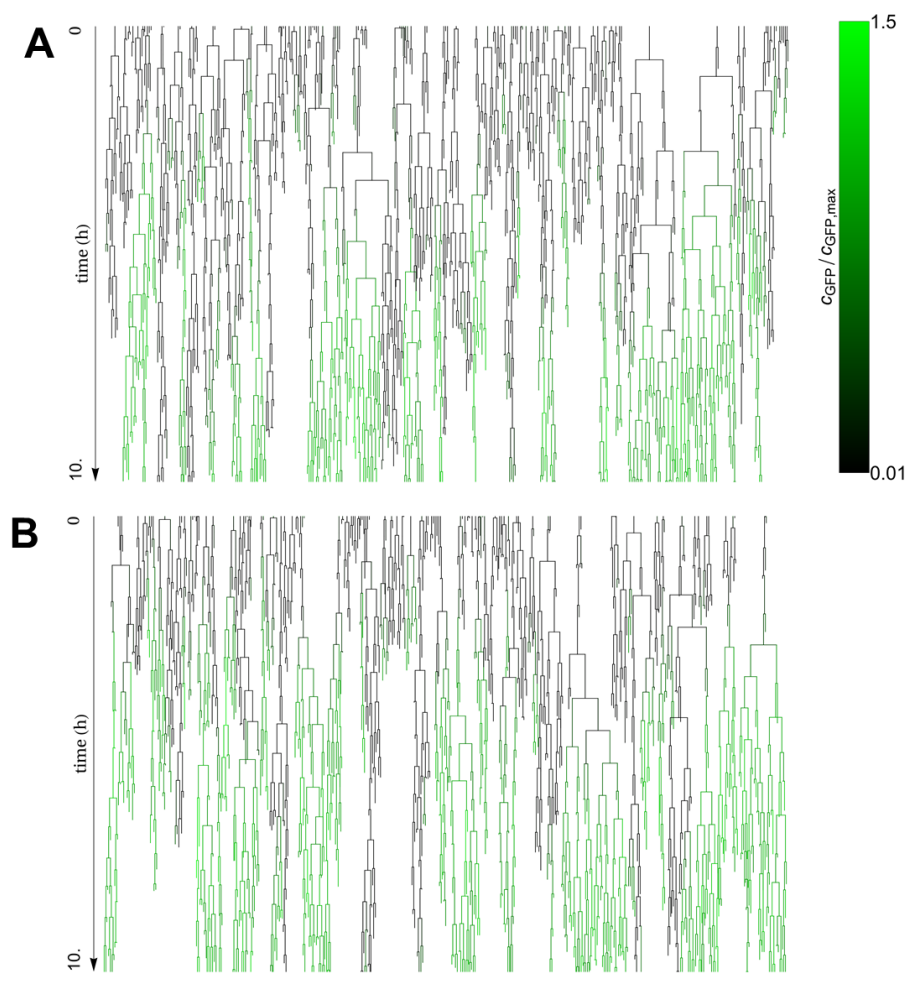

Figure 3.9: Lineage tree of an induced population of cells in the stochastic model. Linage tree of a population of cells induced at a fixed autoinducer concentration $c_{A^{*}}=50 \mathrm{nM}$ for the luxo1 operon (A) and the luxo2 operon (B). Vertical lines represent individual cells and horizontal lines cell division events. The color of the lines changes from black for unactivated cells to green for activated cells, following a color code that depends on the normalized GFP expression. The initial number of cells is 100 and is kept constant during the experiment by deleting cells at random every time a cell divides (truncated vertical lines). The lineage tree shows how the state of the cell is transmitted over generations and reveals that once the operon is activated the transition is "irreversible". 
A
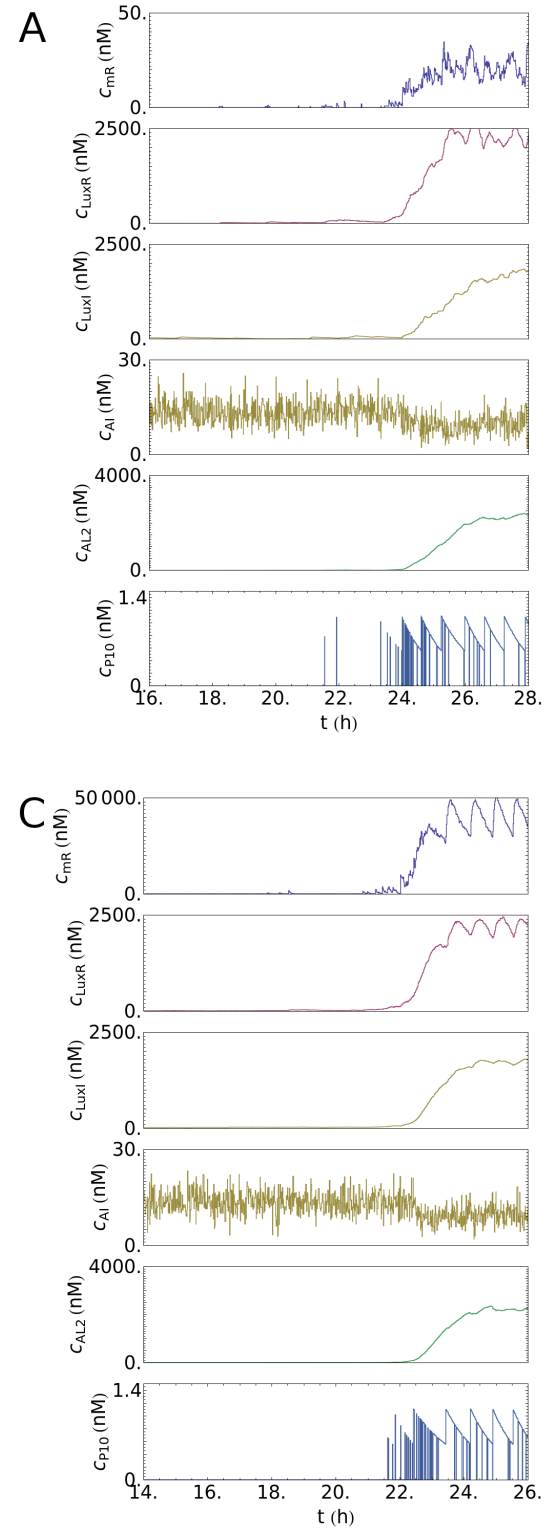

B
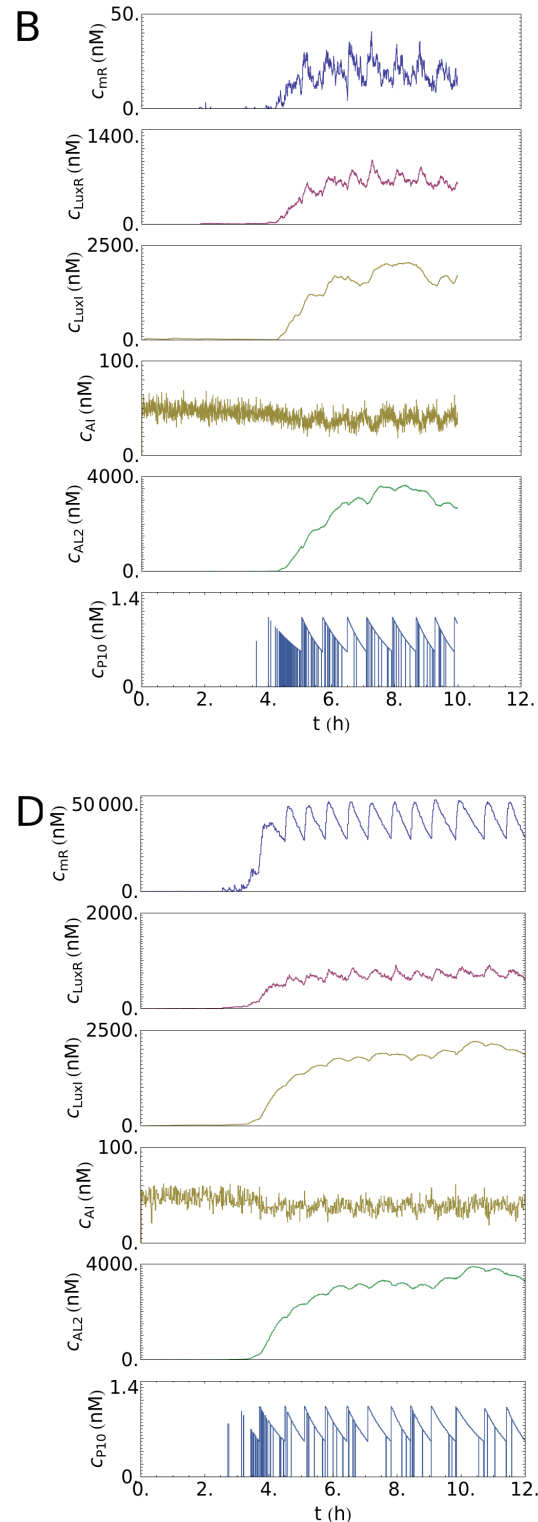

Figure 3.10: Trajectory of chemical species in individual cells. Trajectory of chemical species in the luxo2 operon: LuxR mRNA (mR), LuxR, LuxI, intracellular autoinducer (AI), regulatory complex $\mathrm{LuxR}_{2} \cdot \mathrm{AI}_{2}$ (AL2) and promoter bound to complex (P10), in an individual cell for the following control parameter and burst size values: (A) $c_{A^{*}}=15 \mathrm{nM}, b_{R}=b_{I}=20$, (B) $c_{A^{*}}=50 n M, b_{R}=b_{I}=20,(C) c_{A^{*}}=15 n M, b_{R}=b_{I}=0.01$, (D) $c_{A^{*}}=50 \mathrm{nM}, \mathrm{b}_{\mathrm{R}}=\mathrm{b}_{\mathrm{I}}=0.01$. 
As expected the intrinsic noise decreases the precision of the QS switch with respect to the deterministic case. Still, noise helps cells to become activated before the critical concentration of a fluctuationsfree system under all induction conditions. Moreover, in steady-state conditions the high state is globally achieved before the critical deterministic concentration. This phenomenon is recapitulated in figure 3.6 (bottom) where we plot the population average response for the induction and dilution experiments at steady-state (10o $\mathrm{h}$ induction) for both the deterministic and stochastic models. Notice that the dilution curves of the stochastic model are similar to that of the deterministic model; however, the average transition to the high state occurs at a lower autoinducer concentration due to intrinsic fluctuations.

\subsubsection{The features of the QS switch depends on the transcriptional noise of $\operatorname{LuxR}$}

For the same concentration of the external autoinducer, the stochastic dynamics of the regulatory network arises from the noise at the level of LuxI and LuxR. We now analyze the individual contribution of those by modulating the burst size of LuxR and LuxI, $b_{R}$ and $b_{I}$ respectively. We notice that the burst size modulates the stochasticity levels while maintaining the average protein copy numbers. Figure 3.10 illustrates the effect of changing the burst size by showing individual trajectories of the chemical species obtained for large and small values of this quantity at low and high concentrations of the external autoinducer.

Insight about the activation process can be obtained by computing the mean first passage time (MFPT) for transitions between the low and the high state. Figure 3.11 shows this quantity as a function of $c_{A^{*}}$ and for different values of the burst size of LuxR and LuxI. For the sake of comparison, we also compute the MFPT for the deterministic solution. We note that in that case, the MFPT inside the bistable region is infinite, since the deterministic system cannot spontaneously jump from one stable state to the other. Our results in- 
dicate that changing the burst size of LuxI does not modify the mean first passage time whereas changing the transcriptional noise at the level of LuxR modifies the jumping statistics. Moreover, our results reveal a non-trivial behavior of the MFPT as a function of the concentration of the autoinducer. On one hand, with respect to the activation dynamics, when $\mathrm{c}_{\mathrm{A}^{*}}$ is below $\sim 25 \mathrm{nM}$, an increase in LuxR noise decreases the mean time of the activation. That is, LuxR noise helps cells to get the initial activation quicker. On the other hand, above $\sim 25 \mathrm{nM}$ of autoinducer concentration, the effect is the opposite: an increase in LuxR noise increases the mean jumping time thus slowing down the full cell activation. We observe these effects both for the luxO1 and luxo2 operons. Surprisingly, when the autoinducer concentration is above the critical concentration of the deterministic

Figure 3.11 (following page): Mean first passage time of cell activation for different burst size values. Mean first passage time of cell activation as a function of autoinducer concentration $c_{A^{*}}$ for different values of the burst size for $\operatorname{LuxR}\left(b_{R}\right)$ and LuxI $\left(b_{I}\right)$ and for the deterministic solution: (A) low to high transition MFPT in the luxo1 operon, (B) low to high transition MFPT in the luxo2 operon. The lower (upper) limit of the shaded regions is the $10 \%(90 \%)$ quantile curve of the distribution of FPT for the cases $b_{R}=b_{I}=20$ (blue shaded region) and $b_{R}=b_{I}=0.01$ (green shaded region). The distribution of the FPT for $c_{A^{*}}=100 \mathrm{nM}, b_{R}=b_{I}=20$ is plotted on the side as an example. The MFPT reveals a non-trivial behavior: for low autoinducer concentration noise helps cells to jump quicker to the high state, while for high autoinducer concentration noise slows down the cells activation (see text). Intersections of the quantile $10 \%$ and quantile $90 \%$ curves with a horizontal line at $\mathrm{t}=10 \mathrm{~h}$ indicate the autoinducer concentration for which $10 \%$ of cell trajectories have jumped to the high state (left arrow) and the concentration for which $90 \%$ of cell trajectories have been activated (right arrow). The precision after 1oh of induction is inversely proportional to the width of the region delimited by the arrows, and increases when decreasing the noise in LuxR (see text). Note that in the case of the luxo1 operon, there is no luxI gene and therefore $b_{I}$ cannot be changed. 


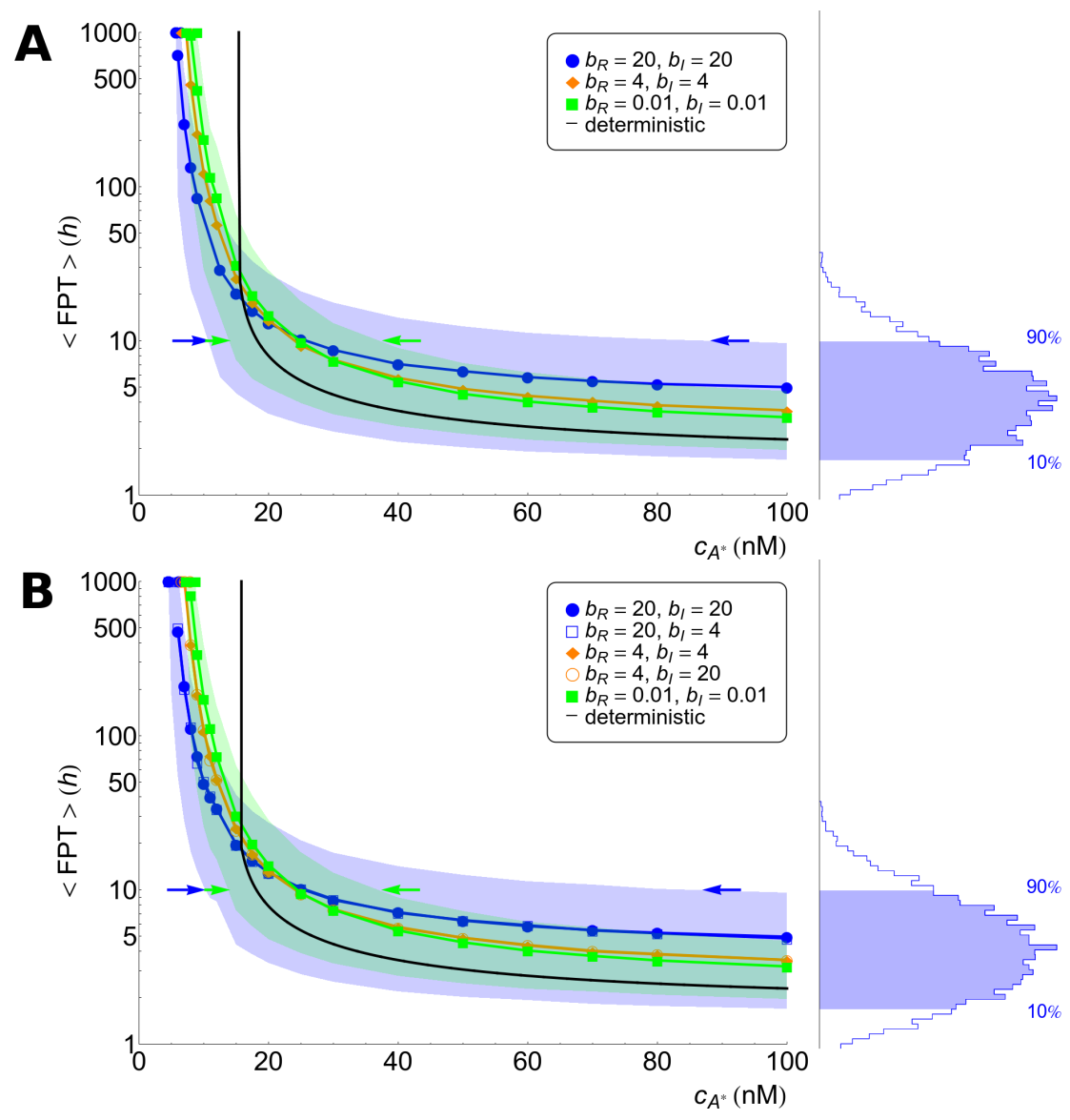


system, $\mathrm{c}_{\mathrm{A}^{*}} \approx 20 \mathrm{nM}$, the stochastic system always takes more time to get activated than the deterministic case (see chapter 4 ).

By computing additional properties of the first passage time probability density we also clarify the behavior of the precision depending on the induction time. In particular, we compute the times $t_{\text {low }}$ and $t_{\text {high }}$ for which, at a given $c_{A *}$ concentration, the probabilities of finding a FPT $<t_{\text {low }}$ and a FPT $>t_{\text {high }}$ are $10 \%$, i.e. the $10 \%$ and $90 \%$ quantiles respectively. The shadings in figure 3.11 delimit these regions for the cases $b_{R}=b_{I}=20$ and $b_{R}=b_{I}=0.01$. The precision of the switch after $n$ hours of induction, is directly related to the width of the shaded region at $\langle\mathrm{FPT}\rangle=\mathrm{n}$ hours: at any given time, this width indicates which is the minimal concentration of autoinducer for getting $10 \%$ of cells already activated and also the concentration beyond which more than $90 \%$ of cells have been activated. Thus, in agreement with figure 3.6, the induction time clearly modifies the precision: it increases (the width decreases) as the induction time becomes larger. Moreover, note that as the LuxR noise weakens the precision increases. Figure 3.12 recapitulates some of these results. There we show the GFP expression probability for the luxO2 operon after 10 hours of induction for different values of the burst size $b_{R}$ and $b_{I}$. Notice that the region of bimodality does not vary when changing the burst size for LuxI. However, decreasing the burst size in LuxR reduces the region of bimodality thus increasing the precision of the switch. Furthermore, the noise at the level of LuxR helps some cells to become activated at lower concentration levels of the autoinducer. Once more, this phenomenon does not depend on the levels of transcriptional noise of LuxI. That is, while the global coordination increases as the transcriptional noise of LuxR decreases, more concentration of the autoinducer is required to start activating cells. Figure 3.11 also suggests that the sensitivity of the precision as a function of the induction time and/or as a function of the stochasticity levels get diminished after $\sim 30$ hours since the width of the shaded region barely varies. Figure 3.13 points towards that direction: under long induction time conditions $(100 \mathrm{~h})$ the precision of the switch remains constant regardless the value of the burst size. All together, these results indicate an interesting and 
A

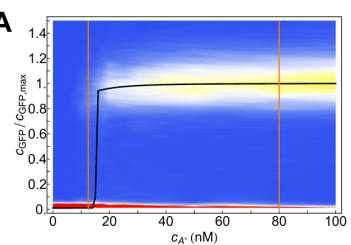

D

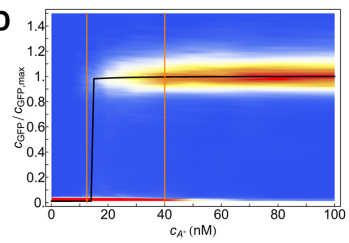

B

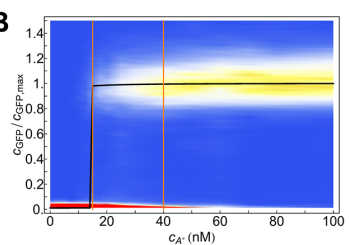

E

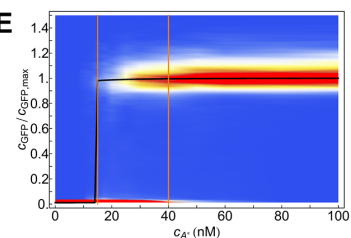

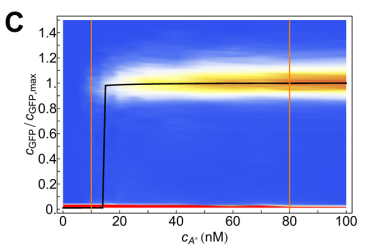

Figure 3.12: Cell response distribution in the transient regime for different burst size values. Cell response distribution (jumping probability) after 10 hours of induction (transient state) at different autoinducer concentrations for the luxo2 operon in the stochastic model, for different burst sizes. Burst size values: (A) $b_{R}=b_{I}=20(B) b_{R}=4, b_{I}=20(C) b_{R}=20, b_{I}=4(D)$ $b_{R}=b_{I}=4(E) b_{R}=b_{I}=0.01$. Width of bistable region: (A) $=60 \mathrm{nM}$ (B) $25 \mathrm{nM}$ (C) $70 \mathrm{nM}$ (D) $27.5 \mathrm{nM}$ (E) $25 \mathrm{nM}$. The black line stands for the normalized concentration of GFP as a function of $c_{A^{*}}$ in the deterministic model at the steady state. The region of bimodality in the transient regime only varies when changing the burst size in LuxR $\left(b_{R}\right)$ and does not depend on the burst size of LuxI $\left(b_{I}\right)$.

counterintuitive role of the transcriptional noise of LuxR in terms of the biological function of the QS switch.

\section{$3 \cdot 5$ DISCUSSION}

The response of bacterial colonies driven by the QS signaling mechanism under noisy conditions has been addressed, in a broad sense, by different authors. In particular, the characterization of the collective response as a synchronization phenomenon where the phenotypic variations can be generically predicted has been proposed [71]. However, this approach requires gene regulatory interactions con- 

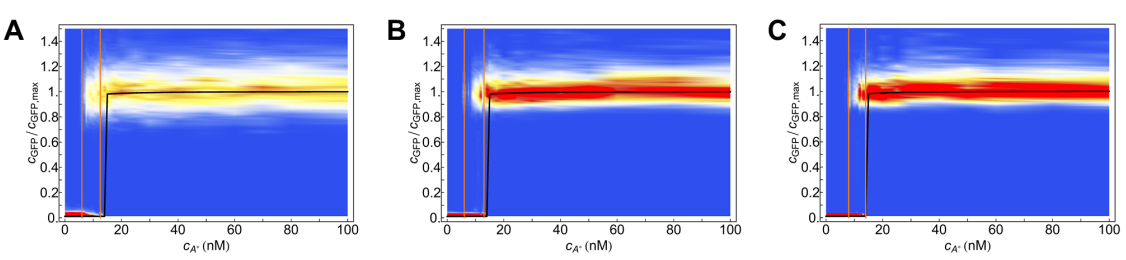

Figure 3.13: Cell response distribution at the steady-state for different burst size values. Cell response distribution at the steady-state (100 h induction), at different autoinducer concentrations for the luxo2 operon in the stochastic model for different burst size values: (A) $b_{R}=b_{I}=20$ (B) $b_{R}=b_{I}=4$ (C) $b_{R}=b_{I}=0.01$. The width of the bistable region, $\approx 7 \mathrm{nM}$, barely depends on the stochasticity levels. The black line stands for the normalized concentration of GFP as a function of $c_{A^{*}}$ in the deterministic model at the steady state.

trolling the QS genes that do not induce bistability and lead to a monostable behavior, e.g. negative feedback loops [205]. Our study focus on strains that display, as the wild-type LuxI/LuxR system do, bistability and, consequently, an alternative method to quantify the phenotypic variability induced by noise was needed. As we have seen, the bistability of the regulatory circuit leads to a bimodal response in the cell population. In this case, fluctuations of individual cells around their current stable state are less relevant at the level of the population than the proportion of cells in each of the stable states ("off" or "on") (see figure 3.7). We have shown that the intrinsic noise, and especially the gene expression noise of LuxR, influences the probability of a cell to jump from the deactivated to the activated state. We propose that the biologically meaningful measure of the synchronization of the cells in this case relies on the homogeneity of the cell response. The new concept we have introduced, the precision, describes the ability of the cell population to respond to the autoinducer concentration in a switch-like manner with a sharp transition and high coordination between cells. At the level of the population average, the precision translates into the sharpness of the 
switch-like response to the autoinducer concentration. A small precision means a bimodal response over a broad range of autoinducer concentrations, producing a graded response at the population level. A high precision means a response that is mainly monomodal and a bimodal response over a narrow range of autoinducer concentrations, providing a switch-like response at the population level.

We have characterized how the precision of the QS switch depends on the stochasticity levels and, importantly, elucidated which noisy component of the LuxI/LuxR regulatory network drives the observed phenomenology. Thus, we have found that under nonstationary conditions, LuxR controls the phenotypic variability and that changing the noise intensity at the level of LuxI has no effect on the precision of the switch. A plausible explanation for this reads as follows. The fluctuations at the level of LuxI are transmitted to the autoinducer. However, the diffusion mechanism, in the regime of high diffusion rates found in many gram-negative bacteria, effectively averages out the fluctuations of the signaling molecule (see chapter 2). This is not possible for LuxR which is kept within the cell. As a consequence the amount of activation complex $(\operatorname{luxR} \cdot A)_{2}$, that is ultimately the responsible for the activation, is driven by the fluctuations of LuxR but not by those of LuxI.

Recent experimental work has measured the bioluminescence levels of individual $V$. fischeri cells at fixed autoinducer concentration [136]. In agreement with our results, the authors observed that cells differed widely in terms of their activation time and luminescence distribution. Interestingly, other experiments have revealed the presence of additional regulatory interactions for controlling the LuxR noise levels. For example, C8HSL molecules, a second QS signal in $V$. fischeri, has been suggested to reduce the noise in bioluminescence output of the cells at low autoinducer concentrations [135]. In the same direction, in $V$. harveyi, the number of LuxR dimers is tightly regulated indicating a control over LuxR intrinsic noise [181]. In fact, wild-type $V$. harveyi strains have two negative feedback loops that repress the production of LuxR [189] and this kind of regulatory circuit is known to reduce noise levels [12]. In this context, our results provide a feasible explanation for the network structure in wild-type 
strains: since noise in LuxR controls the phenotypic variability of the LuxR/LuxI QS systems, bacteria may have evolved mechanisms to control its noise levels. An additional argument in this regard arises from our results about the deactivation of cells: once they are fully induced we do not observe reversibility of the phenotype (FPT larger than $100 \mathrm{~h}$ ). First, these results are in agreement with other switching systems as the galactose signaling network in yeast [ 1$]$ and with theoretical results that explain the highly asymmetric switching dynamics due to stochastic effects (multiplicative noise) [52]. Second, they reveal the importance of additional interactions that regulate negatively luxR in wild-type strains [170, 109, 163] and indicates that synthetic strains as luxo1 and luxo2 summarize many features of the wild-type operon during the activation process but fail to capture some of dynamical aspects of the deactivation phenomenon. Indeed, a theoretical study has shown that while a strong positive feedback is necessary to build a robust switch, it may also induce irreversible transitions and lead to excessively stable states [138]. The addition of a negative feedback can destabilize the stable states and favor the switch transitions.

Most of previous works assume stationary conditions and disregard the role of the cell cycle duration. In our stochastic model, we have shown that the time for reaching a steady state of cell response distribution is much larger than the duration of the cell cycle. This is in agreement with experimental results [198] as well as with another stochastic model of QS transition in Agrobacterium tumefaciens [64]. Notice that in our simulations, the population of cells needs $\sim 30$ hours to reach a steady-state when induced at a concentration of autoinducer of $50 \mathrm{nM}$ and that this time is even greater near the critical concentration of activation (see figure 3.II). In most laboratory experiments studying the QS transition, the typical experimental run or time of culture growth before measurement rarely exceed 20 hours $[123,198,181,106,6]$, after which the expression of genes is assumed to reach a steady-state. While our modeling approach is certainly a simplification of the real genetic network, our results suggest that special care should be taken about transient effects when studying the population-wide QS response. Indeed, bistable gene 
networks are often associated with slow response time compared to graded-response gene networks [159, 185]. As we have seen, stochasticity in the QS bistable switch leads to a large variability of the switching times and to a transient bimodal response. This transient heterogeneity in the population distribution has been proposed to favor cell-fate determination by making the cell decision more reliable compared to a genetic circuit with a graded response and to use the phenotypic heterogeneity as a bet-hedging strategy [12, 185].

Our simulations indicate that non-stationary effects are essential during the activation of the QS response. While speculative, these results can be extrapolated to growing colonies where the cell density is not kept constant. A good supply of nutrients implies short induction times since the concentration of autoinducer will quickly grow (exponentially) as the population size does. According to our results, this fast growing condition decreases the precision of the switch and, consequently, promotes variability at the population level (see figure 3.14, fast growth line). In addition, the full collective activation of the system would require a large population size. On the other hand, if the colony grows in a poor nutrient environment, the system will have time to reach the steady-state and the precision would increase (see figure 3.14, slow growth line). Hence, the variability would be diminished, and full activation would require smaller colony sizes. In addition, modifying the gene expression noise in LuxR would have different effects in these two growth conditions. While increasing the noise in the fast growth condition would increase the variability, it would have little effect in the slow growth condition. In the latter case, cells reach the steady-state and we have seen that the variability hardly changes with the gene expression noise at steadystate. Most phenotypic changes induced by the QS mechanism refer to bacterial strategies for survival and/or colonization. In this context, our results suggest that both the QS activation threshold and the phenotypic variability might depend on the growth rate of the colony and, as a consequence, on the environmental conditions. This is in agreement with recent studies that show that the collective response of a population of cells depends not only on the underlying genetic circuit and the environmental signals, but also on the speed 
of variation of these signals [120]. Moreover, it has been shown that the growth rate, together with the stochasticity in gene expression, can influence the switching rate in the lactose utilization network of Escherichia coli and thus change the transient population distribution [149].

Finally, we have observed a counter-intuitive effect of the gene expression noise in the dynamics of the QS activation. For high concentration of autoinducer (above $\sim 25 \mathrm{nM}$ ) an increase in the noise intensity slows down the mean activation time. This effect is the opposite of what would be expected in the case of a bistable autoactivating switch with additive noise [204]. In the next chapter, I focus on this problem and propose an explanation to this phenomenon: intrinsic noise modifies the bifurcation diagram of the bistable switch and stabilizes the deactivated state of the cells, therefore retaining the cells for more time in the low state.

\subsection{CONCLUSIONS}

Herein I have introduced deterministic and stochastic modeling approaches for describing the core functionality of the LuxI/LuxR regulatory network in quorum sensing systems. I have focused on synthetic constructs, luxo1 and luxo2, that reproduce the behavior of the wild-type system and allow for controlled experiments that have provided quantification of the activation process [198]. The deterministic approach allowed us to estimate different parameters of the model and reproduce the switch-like behavior of the QS network. Our simulations reveal that the interplay between non-stationary and stochastic effects are key and that, for an extended range of autoinducer concentrations, a bimodal phenotypic variability develops such that cells fail to produce a global response. In this context we have introduced the concept of precision of the QS switch, as the inverse of the width of the bimodal phenotypic region.

By computing the statistics of the activation dynamics of cells, we have shown that the QS precision depends on the gene expression noise at the level of LuxR and is independent from that of LuxI. This 


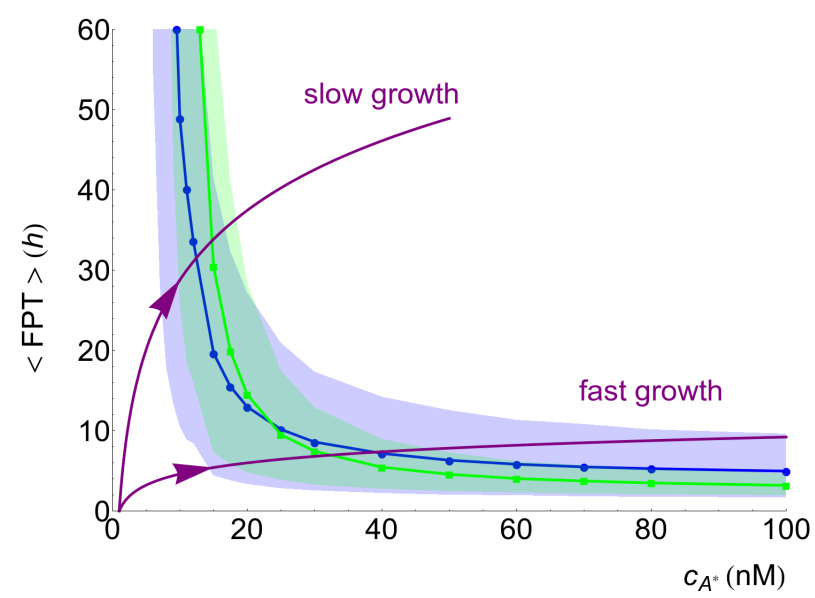

Figure 3.14: The growth rate conditions the phenotypic variability. In the context of a growing colony, the autoinducer concentration increases as the colony does: purple lines show schematically two exponential growth conditions for the autoinducer concentration as a function of time. Our results on the MFPT, valid at fixed autoinducer concentrations, can be extrapolated, qualitatively, to the case of increasing autoinducer levels. Fast growth results in a large cell variability and large critical colony size for achieving a global response, while slow growth produces reduced cell variability and a smaller critical population size. Increasing fluctuations in LuxR have two opposite effects: in the slow growth case, increasing the noise (blue curves: $b_{R}=20$; green curves: $b_{R}=0.01$ ) decreases the critical population size while hardly changing the variability, in the fast growth case, increasing noise increases the critical population size and increases greatly the variability. 
result, together with the experimental evidences on LuxR regulation in wild-type species, suggest that the noise at the level of LuxR controls the phenotypic variability of the LuxR/LuxI QS systems and that bacteria have evolved to control its intensity. In addition, the robust stabilization of the phenotype once is fully induced indicates that, albeit synthetic strains as luxo1 and luxo2 summarize many features of the wild-type operon during the activation process, they fail to capture crucial aspects of the deactivation phenomenon.

Most insight in regards of the effect of LuxR noise on the dynamics of cell activation has been given by the study of the mean first passage time (MFPT). The calculation of the statistics of the first passage time has allowed us to relate the concept of precision of the switch with the variability of the FPT by estimating the 10\% and 90\% quantiles. We have shown that increasing the noise in LuxR decreases the precision in the transient regime while at steady-state the precision does not depend on the burst size of LuxR or LuxI.

In summary, our results indicate that in bacterial colonies driven by the QS mechanism there is a trade-off between the activation onset and a global response due to non-stationary and stochastic effects. On one hand, when compared to a noise-free system, large levels of noise at the level of LuxR imply that cells require smaller autoinducer levels for achieving an activation onset but, at the same time, a global coordinated response requires a substantial autoinducer concentration. On the other hand, if LuxR noise levels are small, the activation onset is shifted toward larger values of the autoinducer concentration but the global response is achieved for smaller concentration values.

Finally, we have also observed a counter-intuitive effect on the mean first passage time for autoinducer concentrations above $20 \mathrm{nM}$ : larger noise in LuxR slows down the mean time of cell activation. From a broader point of view, stochasticity can affect the gene regulatory networks not only in the fluctuations around the stable states, but also by modifying the dynamics and the stable states themselves [162]. In the next chapter, I focus on this problem and study how intrinsic noise modifies the bifurcation diagram of a genetic bistable switch. 


\subsection{INTRODUCTION}

Over the past decade, studies have shown that stochastic effects play an important role in cell differentiation [12]. As introduced in section 1.1, noise allows cells that are exposed to the same environment to choose between different fates, thereby increasing the phenotypic diversity. In this regard, the simplest, non-trivial, regulatory system showing phenotypic multi-stability correspond to a genetic switch with two possible stable solutions: low/high concentrations of a regulatory protein. The core of the genetic circuit underlying bistable systems typically involves a protein that up-regulates its own production, leading to a positive feedback loop. Such a behavior has been found in a number of biological systems, as in the case of the lactose utilization network in E. coli [123], and has been also implemented in synthetic circuits $[56,76,8]$. Moreover, the QS activation circuit in Vibrio fischeri is also based on a positive feedback switch, as seen in chapter 3. Consequently, the characterization of genetic switches is important both for the development of larger and more robust synthetic circuits that use small gene modules with well-defined behaviors [141] and for the understanding of complex processes such as cell differentiation.

The conceptual framework of cell differentiation is rooted in Waddington's ideas about the projection of the genotype space into the phenotype counterpart [59]. Therein phenotypes are associated with attractors, i.e. stable fixed points, in a phase space (the epigenetic landscape) that can be parametrized by the concentration of the molecular species of interest [74]. Interestingly, several studies have 
shown that a stochastic bifurcation diagram (i.e. a stochastic epigenetic landscape) can differ significantly from its deterministic counterpart $[88,162,203,52,79]$. Recent advances in the field include the noise-induced bimodality in the response of the ERK signaling pathway [22] or the increased stability of phenotypic states in bistable systems due to noisy contributions [204, 52, 197]. Moreover, recent studies have clarified the role of different noisy sources for defining the global phenotypic attractor in bistable regulatory systems [79]. Still, despite these efforts, there is a lack of a theoretical formalism to easily understand how those changes in the phenotypic stability are driven by the inherent biochemical fluctuations.

In the context of genetic circuits, a definition of stochastic bifurcation has been previously proposed, based on experimental data [171] or results from gene network models [88, 203]. In general, a stochastic bifurcation is characterized by a qualitative change in one of the observables of the stochastic process. In the case of a bistable system, one may consistently identify two subpopulations of cells whose states are distributed around the two stable states (or attractors) [171]. We follow this approach and define the stochastic system as monostable if its steady state probability distribution is unimodal and bistable if its distribution is bimodal. More complex stochastic bifurcations has been proposed, for example in the case of systems with oscillatory dynamics [203].

\subsection{OBJECTIVES AND SUMMARIZED RESULTS}

In this chapter, I introduce our perturbative theory to analyze how noise modifies the epigenetic landscape. By means of analytical calculation and stochastic simulations, we show how intrinsic noise modifies the bifurcation diagram of gene regulatory processes that can be effectively described by the Langevin formalism. We have applied our findings to a general class of regulatory processes that includes the simplest case that displays a bistable behavior and hence phenotypic variability: the genetic auto-activating switch. Thus, we explain why and how the noise promotes the stability of the low- 
state phenotype of the switch and show that the bistable region is extended when increasing the intensity of the fluctuations. In addition, we examine the role played by biochemical fluctuations with a non-null correlation time and show that, while the effect is lessened, the stochastic modification of the epigenetic landscape also emerges. We compute the rate of activation of the switch and unveil the interplay between the intrinsic noise and the stochastic modification of the energy landscape. While increasing the intrinsic noise leads to a higher switching rate, it also modifies the energy barrier between the low and the high states, resulting in a complex behavior when varying the noise intensity. Our results shed light on the non-trivial behavior of the QS activation dynamics found in chapter 3. This phenomenology is found in a simple one-dimensional model of the genetic switch as well as in a more detailed model that takes into account the binding of the protein to the promoter region. Altogether, we prescribe the analytical means to understand and quantify the noise-induced modifications of the bifurcation points for a general class of regulatory processes where the genetic bistable switch is included. In a general context, our study raises the intriguing question of how biochemical fluctuations redesign the epigenetic landscape in differentiation processes.

The chapter is organized as follows. In the Methods section, I introduce our theoretical approach to analyze the stochastic modification of the epigenetic landscape for a general class of regulatory processes. In the results section, I first apply our finding to a simple model describing a genetic switch. Subsequently, by means of numerical simulations of a more detailed model, I demonstrate that the stochastic stabilization effect is generic for this kind of architecture. Finally, in the Discussion section, I present the main conclusions and discuss about the applicability and relevance of our study. 


\subsection{METHODS}

4.3.1 Stochastic Modification of Bifurcation Points: Perturbative Theory

In the context of gene regulatory circuits, the chemical kinetics formalism that addresses the different processes underlying regulation leads to a Master equation representation [191]. The latter can be approximated by different expansion techniques to an Itō Langevin equation for the concentration of the species [191, 58]. Thus, we start by studying a general stochastic system described by a Itō Langevin equation of one variable $x$ and control parameter $a$ :

$$
\dot{x}=f(x, a)+g(x, a) \bullet \xi(t)
$$

$\xi(t)$ being a Gaussian white noise such that,

$$
\left\langle\xi(t) \xi\left(t^{\prime}\right)\right\rangle=\sigma^{2} \delta\left(t-t^{\prime}\right)
$$

The symbol • indicates that the stochastic process must be interpreted according to Itō.

Under these conditions, the stationary probability density reads [73],

$$
\rho(x, a)=N e^{-\frac{2}{\sigma^{2}} \psi(x, a)}
$$

$\mathrm{N}$ being a normalization constant and,

$$
\psi(x, a)=\sigma^{2} \log [g(x, a)]-\int_{0}^{x} \frac{f(z, a)}{g(z, a)^{2}} d z .
$$

It is easy to show that the extrema of the probability density are located at points that satisfy

$$
h(x, a)=f(x, a)-\sigma^{2} g(x, a) g_{x}(x, a)=0,
$$

where we have used the compact subindex notation for the partial derivatives, e.g. $g_{x x a}=\frac{\partial^{3} g}{\partial x^{2} \partial a}$. 
On the other hand, the inflection points of the probability density satisfy

$$
\begin{aligned}
& 2 f(x, a)\left[f(x, a)-3 \sigma^{2} g(x, a) g_{x}(x, a)\right]+\sigma^{2} g^{2}(x, a)\left[f_{x}(x, a)\right. \\
& \left.+\sigma^{2}\left[3\left(g_{x}(x, a)\right)^{2}-g(x, a) g_{x x}(x, a)\right]\right]=0 .
\end{aligned}
$$

Thus, if there is a stochastic bifurcation point such that a new extrema appears/disappears, the bifurcation points $\left(x^{*}, a^{*}\right)$ must satisfy equations (4.5) and (4.6) simultaneously leading to

$$
h(x, a)=h_{x}(x, a)=0 .
$$

Notice that in the deterministic case, i.e. $\sigma^{2}=0$, the bifurcation points are given by the points $\left(x_{0}, a_{0}\right) \neq\left(x^{*}, a^{*}\right)$, satisfying the equations $f(x, a)=f_{x}(x, a)=0$.

In order to analyze how the bifurcation points vary with to the presence of fluctuations, we assume that, if the noise intensity is small, then the following $\sigma^{2}$-expansion of the points $\left(x^{*}, a^{*}\right)$ holds,

$$
\begin{aligned}
& x^{*}=\sum_{n=0}^{\infty} x_{n} \sigma^{2 n}=x_{0}+\sigma^{2} x_{1}+\mathcal{O}\left(\sigma^{4}\right) \\
& a^{*}=\sum_{n=0}^{\infty} a_{n} \sigma^{2 n}=a_{0}+\sigma^{2} a_{1}+\mathcal{O}\left(\sigma^{4}\right) .
\end{aligned}
$$

Thus, by expanding $f(x, a)$ and $g(x, a)$ in powers of $\sigma^{2}$ and collecting terms, the equations (4.7) read

$$
\begin{aligned}
& h(x, a)=\sum_{n=0}^{\infty} h^{(n)} \sigma^{2 n}= \\
& \quad=f^{0}+\sigma^{2}\left[x_{1} f_{x}^{0}+a_{1} f_{a}^{0}-g^{0} g_{x}^{0}\right]+\mathcal{O}\left(\sigma^{4}\right)=0 \\
& h_{x}(x, a)=\sum_{n=0}^{\infty} h_{x}^{(n)} \sigma^{2 n}= \\
& =f_{x}^{0}+\sigma^{2}\left[x_{1} f_{x x}^{0}+a_{1} f_{x a}^{0}-\left[g^{0} g_{x}^{0}\right]_{x}\right]+\mathcal{O}\left(\sigma^{4}\right)=0,
\end{aligned}
$$


where the superindex 0 indicates that the functions are evaluated at the point $\left(x_{0}, a_{0}\right)$. By solving the conditions $h^{(n)}=h_{x}^{(n)}=0$, we can obtain the corrections $x_{n}$ and $a_{n}$ up to an arbitrary order $n$. As expected, at zero order, $h^{(0)}=h_{x}^{(0)}=0$, we obtain the deterministic conditions for the bifurcation point: $f^{0}=f_{x}^{0}=0$. At order one, $h^{(1)}=h_{x}^{(1)}=0$, we get

$$
\begin{aligned}
& a_{1}=\frac{g^{0} g_{x}^{0}}{f_{a}^{0}} \\
& x_{1}=\frac{\left(g_{x}^{0}\right)^{2}+g^{0}\left[g_{x x}^{O}-\frac{g_{x}^{0} f_{x a}^{0}}{f_{a}^{0}}\right]}{f_{x x}^{O}} .
\end{aligned}
$$

The corrections at order 2 read,

$$
\begin{aligned}
& x_{2}=\frac{1}{6 f_{a}^{3} f_{x x}^{3}}\left(-f_{x a}^{3} f_{x x} g^{2} g_{x}^{2}+f_{a} f_{x a}^{2} g\right. \\
& g_{x}\left(-f_{x x x} g g_{x}+6 f_{x x} g_{x} g_{x x}+2 f_{x x} g g_{x x x}\right)+f_{a}\left(-f_{x a a} f_{x x}^{2} g^{2} g_{x}^{2}+\right. \\
& f_{a}\left(-f_{a} f_{x x x}\left(g_{x}^{2}+g g_{x x}\right)^{2}+2 f_{x x}^{2} g g_{x}\left(2 g_{x} g_{x a}+g_{a} g_{x x}+g g_{x x a}\right)\right. \\
& \left.\left.-2 f_{x x}\left(g_{x}^{2}+g g_{x x}\right)\left(f_{x x a} g g_{x}-3 f_{a} g_{x} g_{x x}-f_{a} g g_{x x x}\right)\right)\right)+f_{x a} \\
& \left(f_{a a} f_{x x}^{2} g^{2} g_{x}^{2}+f_{a}\left(2 f_{a} f_{x x}^{2} g g_{x}\left(g_{a} g_{x}+g g_{x a}\right)\right.\right. \\
& +2 f_{a} f_{x x x} g g_{x}\left(g_{x}^{2}+g g_{x x}\right)+f_{x x}\left(2 f_{x x a} g^{2} g_{x}^{2}+f_{a}\left(g_{x}^{4}-4 g g_{x}^{2} g_{x x}\right.\right. \\
& \left.\left.\left.\left.\left.-6 g_{x}^{3} g_{x x}+g^{2} g_{x x}^{2}-6 g g_{x} g_{x x}^{2}-2 g\left(g_{x}\left(g+g_{x}\right)+g g_{x x}\right) g_{x x x}\right)\right)\right)\right)\right)
\end{aligned}
$$

$$
\begin{aligned}
& a_{2}=-\frac{1}{6 f_{a}^{3} f_{x x}}\left(-f_{x a}^{2} g^{2} g_{x}^{2}+f_{a a} f_{x x} g^{2} g_{x}^{2}+2 f_{a} f_{x a} g g_{x}\right. \\
& \left(3 g_{x} g_{x x}+g g_{x x x}\right)+f_{a}^{2}\left(2 f_{x x} g g_{x}\left(g_{a} g_{x}+g g_{x a}\right)\right. \\
& \left.\left.\quad+\left(g_{x}^{2}+g g_{x x}\right)\left(g_{x}^{2}+g g_{x x}-6 g_{x} g_{x x}-2 g g_{x x x}\right)\right)\right)
\end{aligned}
$$

Our formalism and results can be applied to a general class of regulatory processes with feedback described by the following biochemical reactions:

$$
\varnothing \underset{\mathrm{k}}{\stackrel{\mathrm{m}(\mathrm{x}, \mathrm{a}) \mathrm{V}}{\rightleftharpoons}} \mathrm{X}
$$


where $X$ stands for the regulatory species (number of molecules), $m(x, a)$ is the gene regulatory function describing effective production ( $x$ being the concentration of $X$ ), $k$ the degradation rate and $V$ the cellular volume. It is easy to demonstrate that in this case,

$$
\begin{aligned}
f(x, a) & =m(x, a)-k x \\
g(x, a) & =\sqrt{m(x, a)+k x} \\
\sigma^{2} & =V^{-1} .
\end{aligned}
$$

A bifurcation point leading to multistability will exist if there are at least three non-negative real solutions satisfying the equation $m(x, a)=k x$. In those cases, the modification of the bifurcation points of the deterministic system due to the biochemical noise reads

$$
\begin{aligned}
& a^{*}=a_{0}+\frac{\sigma^{2} k}{m_{a}^{0}}+\mathcal{O}\left(\sigma^{4}\right) \\
& x^{*}=x_{0}+\frac{\sigma^{2}}{2}\left(1-\frac{2 k m_{x a}^{0}}{m_{a}^{0} m_{x x}^{0}}\right)+\mathcal{O}\left(\sigma^{4}\right) .
\end{aligned}
$$

While in terms of $x^{*}$ it is not trivial to envision the sign of the displacement caused by noise, in terms of $a^{*}$ it is easy at least at first order: its sign is prescribed by the putative role played by $a$ in the regulation of species $X$. That is, if a promotes positive regulation (activator), $m_{a}>0$, then $a^{*}>a_{0}$, as in the case of the auto-activating genetic switch (see below). Contrariwise, if $a$ is an inhibitor of production then noise will advance the location of the bifurcation point $\left(a^{*}<a_{0}\right)$.

\subsubsection{Birth and Death Process: Exact Solution}

The formalism and results presented above, apply to the (approximated) Itô Langevin description. Yet, the exact solution of the regulatory processes described by (4.16) can be also obtained. Thus, by comparing the exact epigenetic landscape with that resulting from the Langevin description we can validate the scope of our calcula- 
tions beyond the numerical simulations. Note that the biochemical reactions (4.16) are equivalent to the birth and death processes [207]

$$
\begin{aligned}
& X \rightarrow X+1: V m\left(\frac{X}{V}, a\right) \\
& X \rightarrow X-1: k X
\end{aligned}
$$

The master equation describing this process is

$$
\begin{aligned}
\frac{d p(X, t)}{d t}= & p(X-1, t) V m\left(\frac{X-1}{V}, a\right)+p(X+1, t) k(X+1)- \\
& -p(X, t)\left[V m\left(\frac{X}{V}, a\right)+k X\right]
\end{aligned}
$$

where $p(X, t)$ stands for the probability of having $X$ number of molecules at time $t$. By imposing that at equilibrium the net flux between neighboring states becomes null (detailed balance), the stationary probability, $p(X)$, reads,

$$
p(X)=p(0) \frac{\left(\frac{V}{k}\right)^{X}}{X !} \prod_{Y=0}^{X-1} m\left(\frac{Y}{V^{\prime}}, a\right),
$$

where $p(0)$ is a normalization constant such that $\sum_{X=0}^{\infty} p(X)=1$.

\subsubsection{Non-null Memory Fluctuations}

Single cell level experiments have revealed that intrinsic fluctuations show a "short" correlation time, i.e. white-noise-like [153]. Yet, the white noise is an idealization about the actual behavior of fluctuations since it implies a memoryless process. In order to clarify the consequences of this fact in regard to the modification of the epigenetic landscape, we also examine the role play by colored fluctuations. The so-called Ornstein-Uhlenbeck (OU) process is defined by the stochastic differential equation [73]:

$$
\dot{\eta}=\frac{1}{\tau}\{-\eta+\sigma \xi(t)\},
$$


where $\langle\xi(t)\rangle=0$ and $\left\langle\xi(t) \xi\left(t^{\prime}\right)\right\rangle=\delta\left(t-t^{\prime}\right)$. Under these conditions the mean and the correlation of the OU process read,

$$
\begin{aligned}
\langle\eta(t)\rangle & =0 \\
\left\langle\eta(t) \eta\left(t^{\prime}\right)\right\rangle & =\frac{\sigma^{2}}{2 \tau} e^{-\frac{\left|t-t^{\prime}\right|}{\tau},}
\end{aligned}
$$

where $\tau$ stands for the correlation time (memory) of the fluctuations. In the limit $\tau \rightarrow 0$ the OU process tends to a white noise process with intensity $\sigma^{2}$, that is,

$$
\lim _{\tau \rightarrow 0}\left\langle\eta(t) \eta\left(t^{\prime}\right)\right\rangle=\sigma^{2} \delta\left(t-t^{\prime}\right) .
$$

Importantly, the Wong-Zakai theorem [73] shows that when $\tau \rightarrow 0$, and the OU process is multiplicative in the stochastic differential equation, the right interpretation of the latter is Stratonovich instead of Itō. In this regard, note that the stochastic differential equation,

$$
\begin{aligned}
\dot{x} & =f(x, a)-\frac{\sigma^{2}}{2} g(x, a) g_{x}(x, a)+g(x, a) \circ \xi(t) \\
\langle\xi(t)\rangle & =0 \\
\left\langle\xi(t) \xi\left(t^{\prime}\right)\right\rangle & =\sigma^{2} \delta\left(t-t^{\prime}\right),
\end{aligned}
$$

where $\circ$ indicates that the stochastic integral must be interpreted according to Stratonovich, represents the same stochastic process as equation (4.1). Consequently, the stochastic differential equation,

$$
\dot{x}=f(x, a)-\frac{\sigma^{2}}{2} g(x, a) g_{x}(x, a)+g(x, a) \eta(t),
$$

where $\eta(t)$ stands for the OU process, has the same solution as equation (4.I) in the limit $\tau \rightarrow 0$ and the modification of the epigenetic landscape is the same as in the white noise case (data not shown).

Equation (4.33) cannot be solved analytically for an arbitrary value of $\tau$. However, we can elucidate the modification of the epigenetic landscape in the limit $\tau \rightarrow \infty$, that is, for long correlation times. We notice that in that limit, for a finite noise intensity, the OU process vanishes and the system behaves as the deterministic system [73],

$$
\dot{x}=f(x, a)-\frac{\sigma^{2}}{2} g(x, a) g_{x}(x, a) \text {. }
$$


Therefore, for long correlation times, the bifurcation points are located at points $\left(x^{*}, a^{*}\right)$ satisfying,

$$
\hat{h}(x, a)=\hat{h}_{x}(x, a)=0,
$$

where,

$$
\hat{h}(x, a)=f(x, a)-\frac{\sigma^{2}}{2} g(x, a) g_{x}(x, a) .
$$

Interestingly, these are the same conditions as (4.7) if in equation (4.5) $g(x, a) \rightarrow g(x, a) / \sqrt{2}$ (alternatively if the noise intensity is halved). Thus, at order one, for long correlation times, the correction of the bifurcation points read,

$$
\begin{aligned}
& \hat{a}_{1}=\frac{g^{0} g_{x}^{0}}{2 f_{a}^{0}} \\
& \hat{x}_{1}=\frac{\left(g_{x}^{0}\right)^{2}+g^{0}\left[g_{x x}^{0}-\frac{g_{x}^{o} f_{x a}^{0}}{f_{a}^{0}}\right]}{2 f_{x x}^{0}} .
\end{aligned}
$$

In summary, as the correlation time of the fluctuation increases, the shift effect over the bifurcation points is lessened (see numerical simulation results below). Yet, even in the limit case of infinite memory fluctuations a shift appears and its sign does not depend on the colored character of the noise.

\subsection{RESULTS}

4.4.1 Our perturbative theory reveals the stochastic stabilization of the low state in the autoactivating bistable switch

We now apply our theoretical calculations to a well-characterized system: the auto-activating switch $[76,123]$. In this genetic circuit, a protein forms an oligomer that binds to the promoter region of its own gene and activates its expression (see figure 4.I). As shown elsewhere [52], this regulatory process can be effectively described by the Hill function formalism and leads to the following deterministic equation for the concentration, $x$, of protein: 


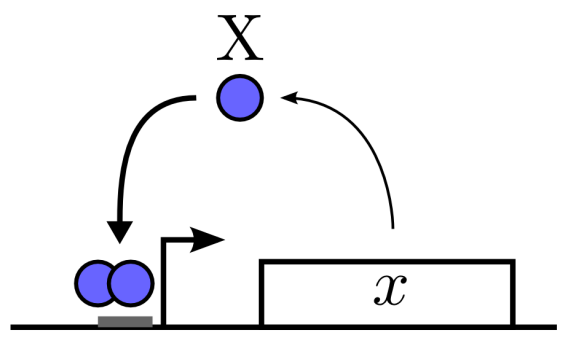

Figure 4.1: Scheme of the genetic auto-activating switch model. The expression of gene $x$ leads to protein $X$ that after oligomerization binds to its own promoter acting as an self-activator.

$$
\dot{x}=r+\frac{a x^{n}}{K_{d}+x^{n}}-k_{5} x
$$

where $r$ is the basal expression rate (due to promoter leakiness), a the maximum production rate (efficiency of the auto-activation), $n$ the cooperativity (oligomerization index), $\mathrm{K}_{\mathrm{d}}$ the concentration of protein yielding half-maximum activation and $k_{5}$ the degradation rate. Notice that the auto-activating regulatory scheme fits within the general class (4.16): $m(x, a)=r+\frac{a x^{n}}{K_{d}+x^{n}}$ being the gene regulatory function. Alternatively, the dimensionless version of equation (4.39) reads

$$
\dot{\tilde{x}}=\tilde{\mathrm{r}}+\tilde{\mathrm{a}} \frac{\tilde{\chi}^{\mathrm{n}}}{1+\tilde{\chi}^{n}}-\tilde{\chi}
$$

with $\tilde{x}=\frac{x}{\sqrt[n]{n} K_{d}}, \tilde{t}=k_{5} t, \tilde{a}=\frac{a}{k_{5} \sqrt[n]{K_{d}}}, \tilde{r}=\frac{r}{k_{5} \sqrt[n]{K_{d}}}$.

If $n \geqslant 2$ and $(3 \sqrt{3})^{-1}>\tilde{r}>0$ then the system exhibits a bistable behavior (phenotypic variability) for a range of values of a. Here we choose $n=2$ and $\tilde{r}=0.12$. Notice that the system has two bifurcation points that define the bistability region. These points correspond to the solutions $\left(x_{0}, a_{0}\right)$ of the polynomial equations,

$$
\begin{aligned}
& f^{0}=\tilde{r}+\frac{\tilde{a}_{0} \tilde{x}_{0}^{2}}{1+\tilde{x}_{0}^{2}}-\tilde{x}_{0}=0 \\
& f_{x}^{0}=\frac{2 \tilde{a}_{0} \tilde{x}_{0}}{\left(1+\tilde{x}_{0}^{2}\right)^{2}}-1=0 .
\end{aligned}
$$


We now examine how the bifurcation diagram (the epigenetic landscape) changes due to the biochemical fluctuations. In particular, we look at the shift of the bifurcation points. The Ito Langevin equation that corresponds to this system reads $[52,58]$

$$
\begin{aligned}
\dot{\tilde{x}} & =f(\tilde{x}, \tilde{a})+g(\tilde{x}, \tilde{a}) \bullet \xi(t) \\
f(\tilde{x}, \tilde{a}) & =\tilde{r}+\tilde{a} \frac{\tilde{x}^{2}}{1+\tilde{x}^{2}}-\tilde{x} \\
g(\tilde{x}, \tilde{a}) & =\sqrt{\tilde{r}+\tilde{a} \frac{\tilde{x}^{2}}{1+\tilde{x}^{2}}+\tilde{x}} \\
\tilde{\sigma}^{2} & =\frac{1}{\tilde{V}}
\end{aligned}
$$

where $\tilde{V}=V \sqrt[n]{K_{d}}$ is the dimensionless volume. By proceeding as described in the previous section, see equations (4.20) and (4.2I), the bifurcation points $\left(\tilde{\chi}^{*}, \tilde{a}^{*}\right)$ read

$$
\begin{aligned}
& \tilde{\mathrm{a}}^{*}=\tilde{\mathrm{a}}_{0}+\tilde{\mathrm{a}}_{1} \tilde{\sigma}^{2}+\tilde{\mathrm{a}}_{2} \tilde{\sigma}^{4}+\mathcal{O}\left(\tilde{\sigma}^{6}\right) \\
& \tilde{\chi}^{*}=\tilde{x}_{0}+\tilde{x}_{1} \tilde{\sigma}^{2}+\tilde{x}_{2} \tilde{\sigma}^{4}+\mathcal{O}\left(\tilde{\sigma}^{6}\right) .
\end{aligned}
$$

The corrections at first order read

$$
\begin{aligned}
& \tilde{\mathrm{a}}_{1}=1+\frac{1}{\tilde{\mathrm{x}}_{0}^{2}} \\
& \tilde{\mathrm{x}}_{1}=\frac{1}{2}+\frac{2}{3 \tilde{x}_{0}^{2}-1} .
\end{aligned}
$$

and the corrections at second order read

$$
\begin{aligned}
& \tilde{\mathrm{x}}_{2}=-\left(3\left(1+\tilde{\mathrm{x}}_{0}^{2}\right)^{6}\right. \\
& +2 \tilde{\mathrm{a}}_{0}\left(1+\tilde{\mathrm{x}}_{0}^{2}\right)^{2}\left(-12+\tilde{\mathrm{x}}_{0}\left(7+3 \tilde{\mathrm{x}}_{0}\left(16+\tilde{\mathrm{x}}_{0}\left(2+\tilde{\mathrm{x}}_{0}\left(-12+5 \tilde{\mathrm{x}}_{0}\right)\right)\right)\right)\right) \\
& +\tilde{\mathrm{a}}_{0}^{2}\left(1+\tilde{\mathrm{x}}_{0}\left(-24+\tilde{\mathrm{x}}_{0}\left(2+3 \tilde{\mathrm{x}}_{0}(8\right.\right.\right. \\
& \left.\left.\left.\left.\left.+3 \tilde{\mathrm{x}}_{0}\left(6+24 \tilde{\mathrm{x}}_{0}-24 \tilde{\mathrm{x}}_{0}^{3}-7 \tilde{\mathrm{x}}_{0}^{4}+6 \tilde{\mathrm{x}}_{0}^{6}\right)\right)\right)\right)\right)\right) / \\
& \quad\left(12 \tilde{\mathrm{a}}_{0}^{2} \tilde{\mathrm{x}}_{0}\left(1+\tilde{\mathrm{x}}_{0}^{2}\right)^{2}\left(-1+3 \tilde{\mathrm{x}}_{0}^{2}\right)^{3}\right) \quad(4 \cdot 51)
\end{aligned}
$$




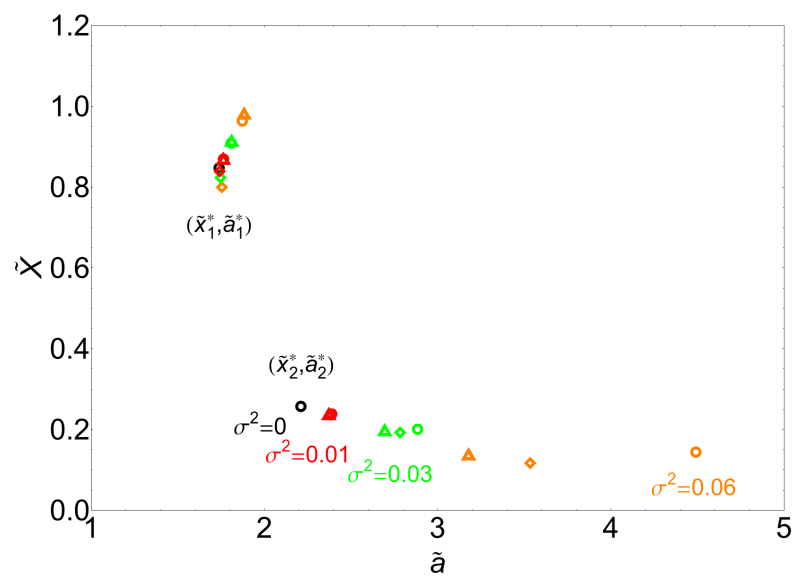

Figure 4.2: Noise-induced shift of the bifurcation points for the simple genetic switch. Change in the position of the bifurcation points $\left(\tilde{x}_{1}^{*}, \tilde{a}_{1}^{*}\right)$ and $\left(\tilde{x}_{2}^{*}, \tilde{a}_{2}^{*}\right)$ for different values of noise intensity: $\tilde{\sigma}^{2}=0$ (black symbols), $\tilde{\sigma}^{2}=0.01$ (red symbols), $\tilde{\sigma}^{2}=0.03$ (green symbols) and $\tilde{\sigma}^{2}=0.06$ (orange symbols). Numerical exact solution (circles), first order approximation (triangles) and second order approximation (diamonds). The biochemical fluctuations shift the position of both bifurcation points but the effect for $\tilde{\mathrm{a}}_{2}^{*}$ is more noticeable and widens the bistability region that ultimately promotes the stability of one phenotype.

$$
\begin{aligned}
& \tilde{\mathrm{a}}_{2}=-\frac{1}{12 \tilde{\mathrm{a}}_{0} \tilde{\mathrm{x}}_{0}^{4}\left(1+\tilde{x}_{0}^{2}\right)^{3}\left(-1+3 \tilde{\mathrm{x}}_{0}^{2}\right)}\left(\left(1+\tilde{\mathrm{x}}_{0}^{2}\right)^{5}\right. \\
& +2 \tilde{\mathrm{a}}_{0} \tilde{\mathrm{x}}_{0}\left(1+\tilde{\mathrm{x}}_{0}^{2}\right)^{2}\left(2+\tilde{\mathrm{x}}_{0}\left(12+\tilde{\mathrm{x}}_{0}\left(1+3\left(-4+\tilde{\mathrm{x}}_{0}\right) \tilde{\mathrm{x}}_{0}\right)\right)\right) \\
& \left.+\tilde{\mathrm{a}}_{0}^{2} \tilde{\mathrm{x}}_{0}^{2}\left(3+\tilde{x}_{0}\left(24+\tilde{x}_{0}\left(5+3 \tilde{\mathrm{x}}_{0}\left(16-3 \tilde{x}_{0}\left(-1+\tilde{x}_{0}\left(8+\tilde{x}_{0}\right)\right)\right)\right)\right)\right)\right) .
\end{aligned}
$$

Figure 4.2 shows, in agreement with our analytical calculations, the location of the bifurcation points as a function of the noise intensity $\tilde{\sigma}^{2}$, at first (triangles) and second (diamonds) orders as well as the exact solutions (circles) (see also figure 4.3 bottom inset). Note that in terms of $\tilde{a}^{*}$ the correction due to the noise is always positive. Consequently both bifurcation points, those defining the bistable region, are shifted to the right. Moreover, the shift largely increases as 


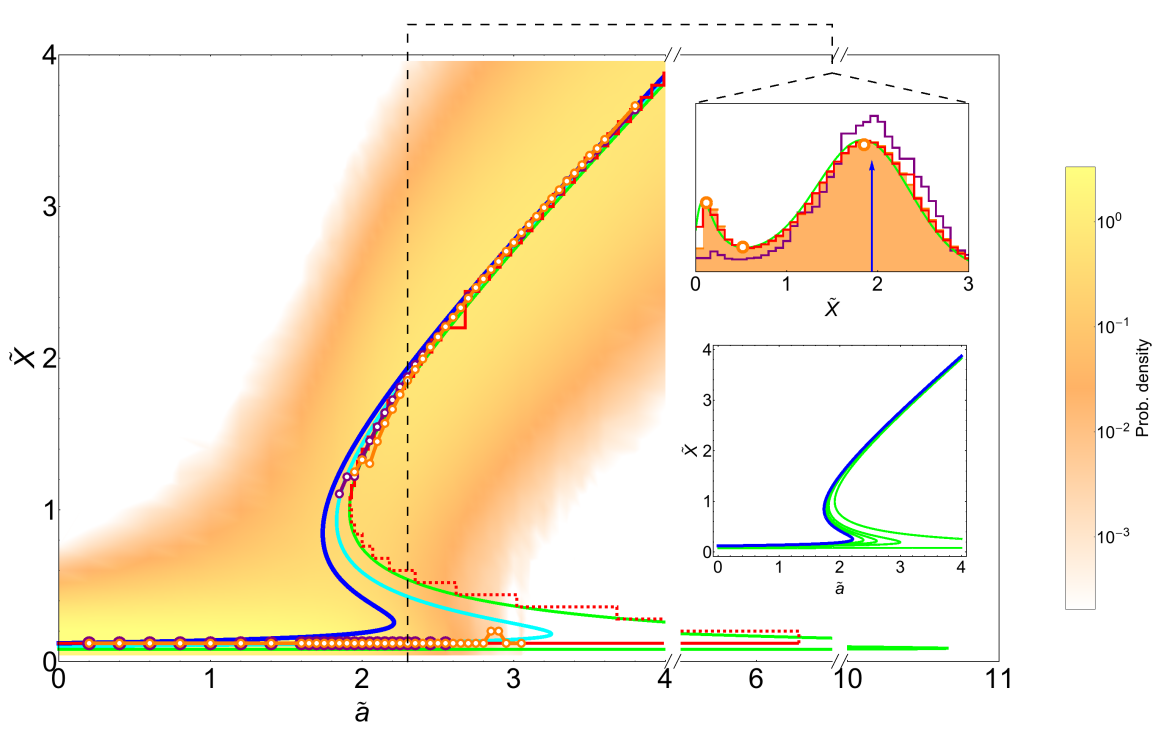

Figure 4.3: Bifurcation diagram of the simple genetic switch. Deterministic system (blue line), white-noise stochastic system (Langevin: green line; exact solution: red line). The bifurcation diagram of a system with colored fluctuations in the limit $\tilde{\tau} \rightarrow \infty$ is also depicted (cyan line). In all cases $\tilde{V}=12.5$. The results from stochastic simulations are in agreement with the analytical results, as can be seen by the detected peaks (orange circles) of the probability distribution of $\tilde{x}$ at steady state (color code, logarithmic scale). The numerical simulations for a non-null correlation time noisy system, with $\tilde{\tau}=0.1$, indicate that the effect is lessened when memory is considered (purple circles). The top inset reveals that the probability distributions obtained in numerical simulations (orange histogram) are in perfect agreement with the exact solution (red line) and the Langevin description (green line), $\tilde{a}=2.3$. For that value of the control parameter the deterministic system only have one stable solution and the probability distribution corresponds to a Dirac delta (blue arrow). When the correlation time of the noise is not null, $\tilde{\tau}=0.1$, the stability of the low state decreases with respect to the white noise case (purple line). The circles in the inset denote the extrema as detected by the Gaussian peak detection algorithm. Bottom inset: increasing noise (decreasing volume) clearly extends the stable branch of the low state, an effect that we call stochastic stabilization: $\tilde{V}=100,50,30$ and 12.5 . 
$\tilde{x}_{0}$ approaches to zero, and the bistable region widens with respect to the deterministic system. In addition, the low state, for which $\tilde{x}_{0} \gtrsim 0$, has a negative correction in terms of $\tilde{x}^{*}$, i.e. $\tilde{x}^{*}<\tilde{x}_{0}$. Altogether, our calculations indicate that one of the states (the low protein concentration one) becomes more stable due to the biochemical noise. We call this effect the stochastic stabilization of a phenotypic state. Alternatively, this phenomenon can be interpreted as a noise-induced bistability since there is a range of values of the control parameter for which the stochastic system displays a bistable behavior in opposition to the monostable response of the deterministic system.

Figure 4.3 shows the analytical bifurcation diagrams for the deterministic and the stochastic cases. In the stochastic cases, with regard to the Langevin approximation, we define the stable and unstable branches by means of the extrema of the probability distribution, i.e. by numerically solving the condition (4.5) and, in the case of the exact analytical solution, equation (4.25), by numerically finding the extrema of $p(X)$. The results support the stochastic stabilization phenomenon: compared with the deterministic system the low protein concentration state becomes stable for a larger range of values of the control parameter as the noise intensity increases. Moreover, our results validate the Langevin approximation since is in good agreement with the exact solution.

In order to gain more insight into the stabilization phenomenon, we perform stochastic simulations of equations (4.1) and (4.33). In these cases, the position of the maxima are computed by using a Gaussian peak detection algorithm over the numerical probability distributions obtained in the simulations. On one hand, in the white noise case, the positions of the maxima show a good agreement with the analytical results. However, our simulations reveal that despite noise extends the low state stability to higher values of a , the probability of residing at the low state quickly drops when increasing the control parameter. This is the reason why the detected peaks of the low state from the simulations do not extend as far as the stable branch of the low state computed from the analytical calculations. On the other hand, in the case of the colored noise $(\tilde{\tau}=0.1)$, the simulations confirm the analytical calculations when the biochemi- 
cal fluctuations are not memoryless: for the same noise intensity of the white noise case, the effect is lessen, yet present and the low state becomes stabilized.

The noise-induced bistable behavior is revealed by the bimodal shape of the stationary probability density in opposition to the deterministic system for which the only stable solution is the high state (figure 4.3 top inset). The analytical distribution computed from (4.3) agrees with both the exact solution and the distribution computed from the stochastic simulations. Notice that when colored noise is considered the stochastic stabilization effect is lessened as revealed by the drop of the maximum that correspond to the low state.

Figure 4.4 shows stochastic trajectories for $\tilde{a}=2.3$ when the fluctuations are white. At this value, the low state is unstable in the deterministic system and the only plausible phenotype is the high state. Indeed, when the volume is large (low noise intensity) the system is monostable and stays at the high state $(\tilde{x} \sim 2)$. However, when the volume is small (high noise intensity) the system exhibits a bistable behavior, jumping between the high state and the low state $(\tilde{x} \sim 0.1)$. In order to show that the residence time at the low state is large enough and, as a result, noticeable as a phenotype, we indicate in figure 4.4 the characteristic duration of a bacterial cell cycle (40 minutes) in dimensionless time units, under two different conditions of the protein degradation rate: stable proteins with an effective degradation driven by the cellular growth (dilution), $k_{5}=\log (2) / 40 \mathrm{~min}^{-1}$, and unstable proteins with fast degradation induced by tagging [113], $\mathrm{k}_{5}=(\log (2) / 40+1.0) \mathrm{min}^{-1}$.

\subsubsection{The stochastic stabilization effect is also found in a more detailed model of autoactivating switch}

Our results towards the understanding of the modification of the phenotypic landscape due to the biochemical fluctuations are based on a simplified view of the regulatory process (the genetic switch) described by a single variable. However, one might wonder if our results are applicable when a more detailed model is considered, that 


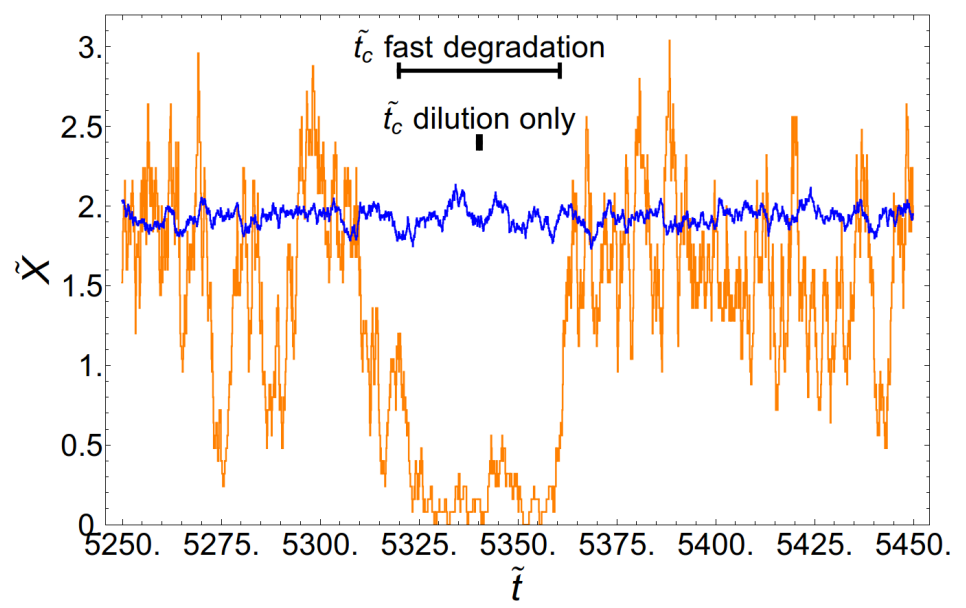

Figure 4.4: Trajectories for the simple genetic switch for small and large volumes. Trajectories from stochastic simulations of the simple genetic switch model for small volume $\tilde{V}=12.5$ (orange line) and large volume $\tilde{V}=1000$ (blue line) for $\tilde{a}=2.3$. For this value of parameter $\tilde{a}$, the system is monostable and stays at the high state $(\tilde{x} \sim 2)$ when the noise intensity is small (large volume), while it is bistable when the noise intensity is high (small volume) and jumps between the low state $(\tilde{x} \sim 0.1)$ and the high state $(\tilde{x} \sim 2)$. The black bars indicate the characteristic duration of a bacterial cell cycle $\left(t_{c}=40\right.$ minutes) in dimensionless time units under two different conditions of the protein degradation rate and show that the residence time at the low state is large enough to be noticeable as a phenotype: stable proteins driven by dilution effects $\left(k_{5}=\log (2) / 40 \mathrm{~min}^{-1}\right)$ and unstable proteins with fast degradation induced by tagging $\left(k_{5}=(\log (2) / 40+1.0) \min ^{-1}\right)$. 
is, if our predictions are an artifact due to an oversimplified mathematical description. In order to address this question, we consider a more detailed, yet equivalent, model of the genetic switch using the chemical kinetics formalism. In particular, we take into account the basal expression rate, the binding/unbinding events of the protein oligomer to the promoter, an effective transcription/translation rate, and the protein degradation:

$$
\begin{gathered}
\varnothing \underset{\mathrm{k}_{5}}{\stackrel{\mathrm{rV}}{\rightleftharpoons} \mathrm{X}} \\
\mathrm{P}+\mathrm{n} X \underset{\mathrm{k}_{3} / \mathrm{V}^{\mathrm{n}}}{\stackrel{\mathrm{k}_{4}}{\rightleftharpoons}} \mathrm{P}^{*} \\
\mathrm{P}^{*} \stackrel{\mathrm{k}_{2}}{\longrightarrow} \mathrm{P}^{*}+\mathrm{X}
\end{gathered}
$$

where $X$ stands for the protein (number of molecules), $n$ its oligomerization index and $\mathrm{P} / \mathrm{P}^{*}$ the unbound/bound states of the promoter. These reactions lead to the following deterministic description in terms of ordinary differential equations describing the concentration of chemical species,

$$
\begin{aligned}
\dot{\mathrm{p}} & =k_{4} \mathrm{p}^{*}-\mathrm{k}_{3} p x^{n} \\
\dot{p^{*}} & =-k_{4} p^{*}+k_{3} p x^{n} \\
\dot{x} & =-k_{5} x+r+n\left(k_{4} p^{*}-k_{3} p x^{n}\right)+k_{2} p^{*} .
\end{aligned}
$$

Binding/unbinding events are fast reactions compared to the protein production and the degradation, i.e. $k_{3}, k_{4} \gg r, k_{2}, k_{5}$. Thus, a quasi-steady state approximation can be implemented such that $\dot{p}=\dot{p}^{*} \simeq 0$. The latter combined with the conservation law of the total promoter concentration, $p+p^{*}=p_{0}$, leads to the following equation,

$$
\dot{x}=r+\underbrace{k_{2} P_{0}}_{a} \frac{x^{n}}{\underbrace{k_{4} / k_{3}}_{K_{d}}+x^{n}}-k_{5} x .
$$

Therefore, assuming that the binding/unbinding of the protein to the DNA are fast reaction, this model leads to the same deterministic equation as in the simple genetic switch model. However, its 
stochastic description in terms of the set of reactions 4.53 to 4.55 is far more complex that equation (4.43) even considering that binding/unbinding are fast events since $\mathrm{P}$ and $\mathrm{X}$ are correlated quantities and each species exhibits a fluctuating dynamics [79]. Then, we perform stochastic simulations of reactions 4.53 to 4.55 using the Gillespie algorithm [57] and apply the peak detection method to elucidate the bifurcation changes in the epigenetic landscape.

In order to reduce the number of parameters, we use the same definition of dimensionless variables as above. Compared to the simplified model, the detailed model has two additional parameters, $\tilde{k}_{2}$ and $\tilde{k}_{4}$. Parameter $\tilde{k}_{2}$ is related to the control parameter $\tilde{a}$ by the relation $\tilde{k}_{2} P_{0} \equiv \tilde{a}$. In order to change $\tilde{a}$, we vary the value of $\tilde{k}_{2}$ and keep fixed the DNA copy number $P_{0}=1$. In our simulations the value of $\tilde{k}_{4}$ is fixed $\left(\tilde{k}_{4}=1000\right)$ and ensures that equation (4.59), and consequently the deterministic bifurcation diagram, applies when fluctuations are neglected. Yet, when considering the noise, the differences between the simple and the detailed stochastic model are noticeable by examining the stationary probability distributions (see figure 4.5 ). Nonetheless, in agreement with our theoretical approach, the bifurcation diagram, figure 4.6 , shows that noise promotes the stability of the low state compared to the deterministic system. However, the stochastic stabilization effect is smaller than in the simple genetic switch model. For example, for a volume $\tilde{V}=12.5$ the maximum in the distribution corresponding to the low state can be detected up to the value $\tilde{\mathrm{a}}=2.8$ for the detailed model, while in the simple model it can be detected up to $\tilde{a}=3.1$.

\subsubsection{First passage time simulations reveal the interplay between intrinsic noise and stochastic modification of the energy landscape on the dynamics of the autoactivating switch}

The mean first passage time (MFPT) in a stochastic bistable system reflects the time that the system remains in the neighborhood of a stable state before jumping to the other stable state. In the case of the simple model, we can calculate analytically the MFPT in the Fokker- 


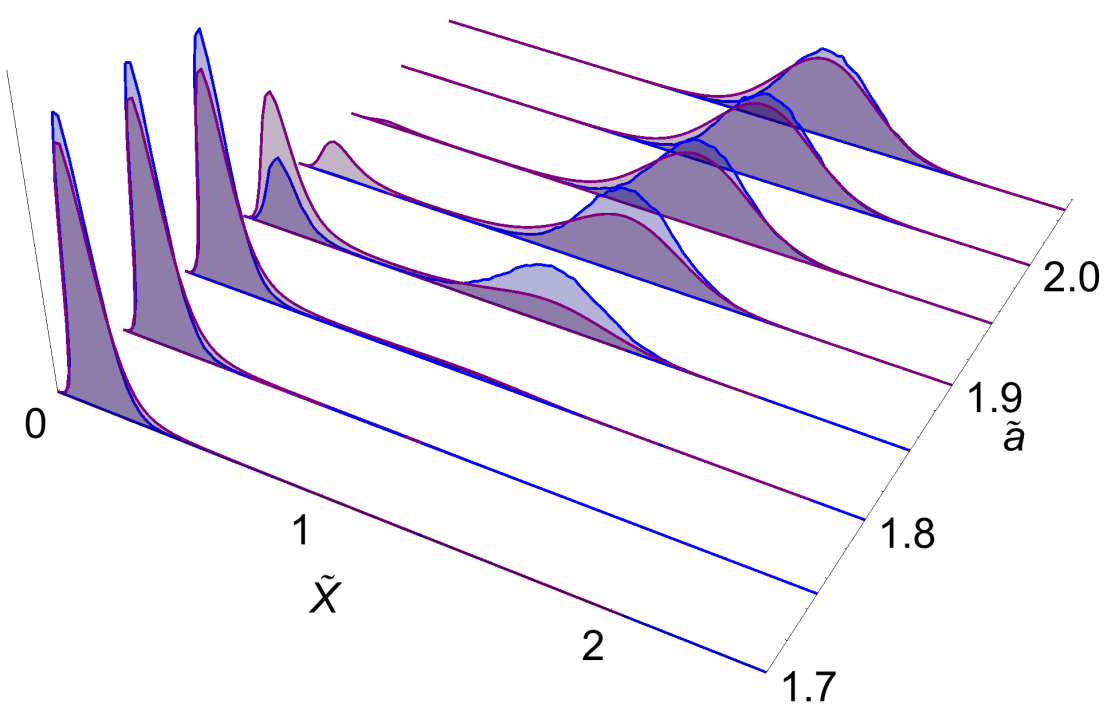

Figure 4.5: Steady state probability distribution for the simple and detailed models. Probability distribution at steady state for volume $\tilde{V}=100$ for the simple (purple filled curves) and the detailed (blue filled curves) genetic switch models, for different values of the control parameter ã. The distributions match well except in the region where distributions are bimodal and highlight the fact that while the deterministic description is the same in both models, the stochastic one is not. 


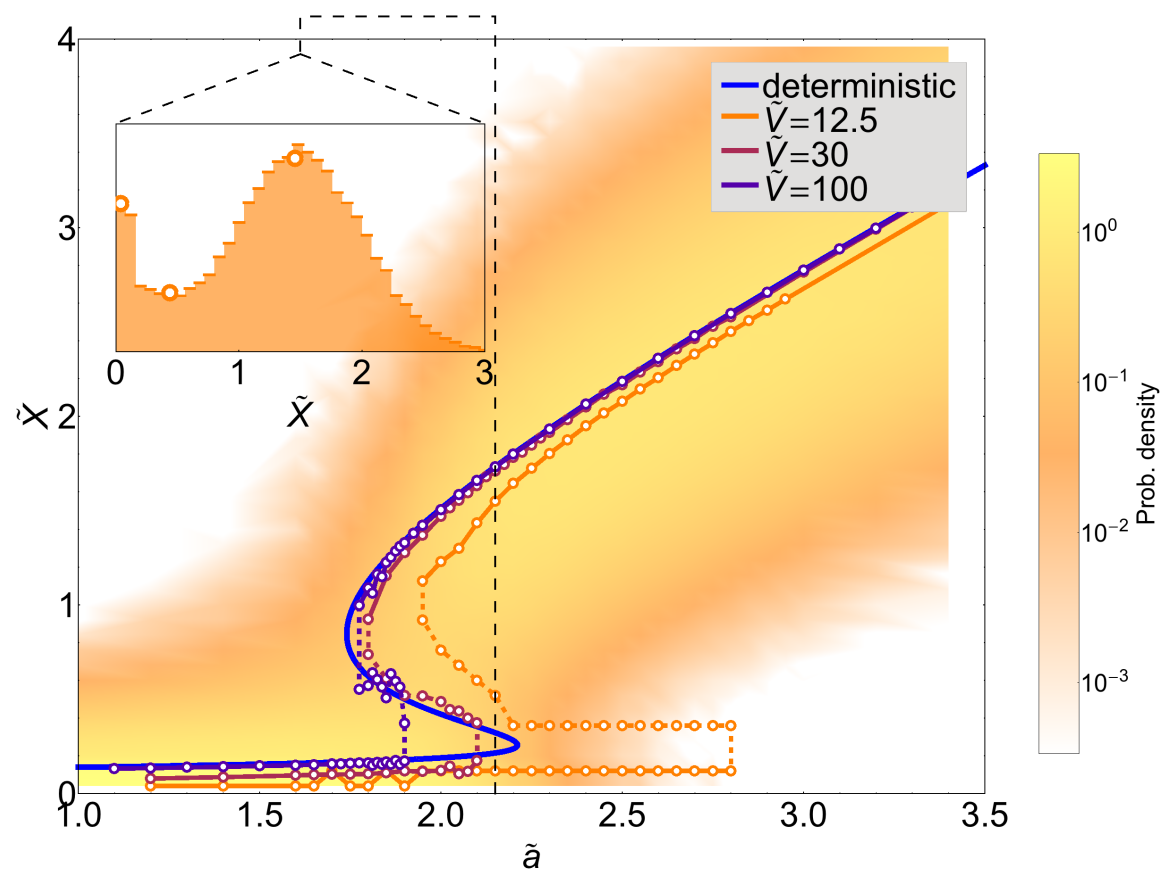

Figure 4.6: Bifurcation diagram of the detailed genetic switch. Bifurcation diagram of the detailed genetic switch with fast protein-DNA binding/unbinding, The results from stochastic simulations at low volume $\tilde{V}=12.5$ shows that noise induces the same stochastic stabilization effect as in the simple genetic switch model, as can be seen by the position of the peaks and minima of the distribution from the simulations (orange circles) compared to the deterministic system (blue solid line). The same Gaussian peak detection algorithm is used in order to detect the extrema in the steady-state distribution (inset, $\tilde{a}=2.15$ ). Color code denotes the probability distribution from stochastic simulations for $\tilde{V}=12.5$ in logarithmic scale. The stochastic stabilization effect is less pronounced than in the simple model and is only detectable for low volumes. At intermediate volume (light purple circles, $\tilde{V}=30$ ) and large volume (dark purple circles, $\tilde{V}=100$ ), the bistable region of the stochastic system is smaller than in the deterministic case. 
Planck description corresponding to the Langevin equation (4.43). With a reflecting barrier at $\tilde{x}=0$ and an absorbing barrier at the maximum of the Fokker-Planck potential $\psi\left(\tilde{x}_{\max }\right)$, the MFPT reads [55]

$$
\begin{aligned}
T_{\text {low }} & =2 \int_{\tilde{x}_{\text {low }}}^{\tilde{x}_{\max }} \frac{d y}{\phi(y)} \int_{0}^{y} \frac{\phi(z)}{\sigma^{2} g(z)^{2}} d z \\
\phi(\tilde{x}) & =e^{\int_{0}^{\tilde{x}} d x^{\prime} \frac{2 f\left(x^{\prime}\right)}{\sigma^{2} g\left(x^{\prime}\right)^{2}}}
\end{aligned}
$$

We also computed the MFPT by means of Gillespie simulations, starting from the maximum of the steady-state distribution of the exact solution (4.25) and recording the time needed to reach the minimum. Panel A in figure 4.7 shows the MFPT as a function of the control parameter a for different volumes. The MFPT obtained with the Gillespie simulations are in agreement with the analytical calculations of the Fokker-Planck approximation. Notice that the MFPT of the simulations exhibit some steps at certain values of parameter $\tilde{a}$ for low volumes. This is due to the discrete shifts in the position of the extrema in the steady-state distribution when changing the control parameter ã. Because there are only a few molecules in the low state and in the unstable state, a change in the integer value of its positions induces a step in the resulting MFPT. Depending on the value of $\tilde{a}$, the MFPT increases or decreases with the intrinsic noise intensity. Here, two different contributions must be identified to understand the behavior of the first passage time as a function of the control parameter $\tilde{a}$ and the intensity of the intrinsic noise $\sigma^{2}$ (inversely proportional to the volume $\tilde{V}$ ). First, we expect that if the energy landscape was fixed, an increase in the intensity of the intrinsic noise would lead to a higher switching rate, as shown in the case of the autoactivating switch [88]. This is what we observe when the control parameter $\tilde{a}$ is close to the deterministic bistable region. However, we observe the opposite effect for higher values of ã: an increase in the noise intensity decreases the switching rate. We hypothetize that the second contribution is due to the stochastic modification of the energy landscape. Indeed, we have seen that the bifurcation diagram is modified when changing the intensity of 

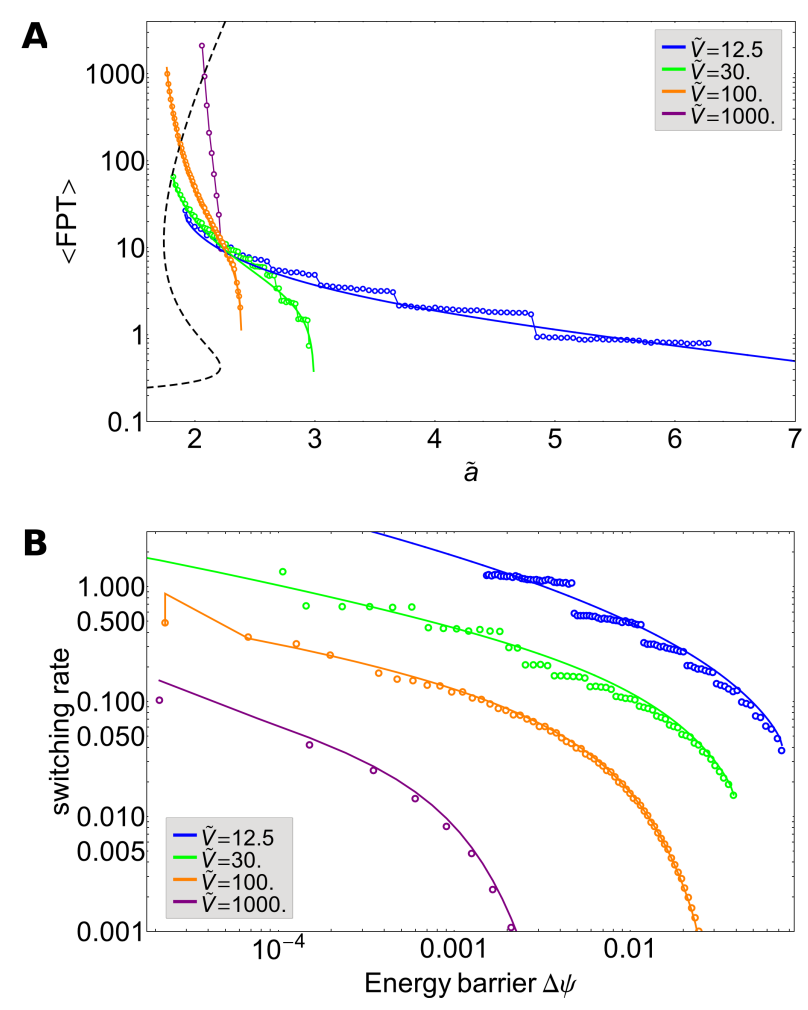

Figure 4.7: First passage time and switching rate for the simple model. (A) Mean first passage time (MFPT) for the simple model of genetic switch from the low stable state to the unstable state, defined as the maximum and minimum of the steady-state distribution: analytical solution of the Fokker-Planck description (solid lines) and Gillespie simulations (circles), for different volume sizes (see legend). The deterministic bifurcation curve (dashed line) is depicted with an arbitrary y-scale in order to visualize the deterministic bistable region. For decreasing volume (increasing noise), the bistable stochastic region increases. Depending on the value of $\tilde{a}$, the MFPT increases or decreases with the intrinsic noise intensity, due to the combined effect of the modification of the bistable region and the change in the escape rate due to noise. (B) Switching rate (inverse of the MFPT) from the low state to the unstable state in the simple model as a function of the energy barrier $\Delta \psi$. The energy barrier is the difference of potential $\psi\left(\tilde{x}_{\max }, \tilde{\mathrm{a}}\right)-\psi\left(\tilde{\mathrm{x}}_{\text {low }}, \tilde{\mathrm{a}}\right)$ from the solution (4.3) of the Fokker-Planck approach. The switching rate clearly increases with noise intensity for all values of the energy barrier. 
noise. Therefore, for a fixed value of parameter ã, these two contributions determine the behavior of the MFPT. In order to unveil the importance of these two contributions, we plot on figure $4.7 \mathrm{~B}$ the switching rate (inverse of the MFPT) as a function of the energy barrier $\Delta \psi$. By doing so, we can compare the switching rate behavior when changing the noise intensity while keeping fixed the energy landscape. The energy barrier is the difference of potential $\psi\left(\tilde{x}_{\max }, \tilde{\mathrm{a}}\right)-\psi\left(\tilde{x}_{\text {low }}, \tilde{\mathrm{a}}\right)$ from the solution (4.3) of the Fokker-Planck approach. For the same value of the energy barrier, the switching rate increases with the intensity of the intrinsic noise, as expected. However, in terms of the control parameter a, the range of bistability is shifted to the right and the energy barrier is modified when increasing the noise. The combination of these two effects result in the observed behavior of the MFPT as a function of $\tilde{a}$, in particular the MFPT curves for different volumes crossing each other. In the case of the detailed model, the results are similar (see figure 4.8), however the bistable region is much narrower and this difficults the comparison for the different volumes. Moreover, the detailed model is multi-dimensional and does not allow for the definition of an energy potential, as in the case of the simple model.

In order to analyze the dynamics of the switch not only in the bistable region but also for larger values of the control parameter, we computed the mean time that the system needs to reach a position close to the high state, at $0.9 \tilde{x}_{\text {high }}$, starting from the low state (see figure 4.9). The low and high stable states are defined as the maxima of the steady-state distribution of the Fokker-Planck description. This quantity represents the mean time that a cell would need to activate the genetic switch starting from the unactivated state. Depending on the value of the control parameter $\tilde{a}$, the maximum production rate of the protein, we observe two opposite effects of the intrinsic noise: for $\tilde{\mathrm{a}} \lesssim \tilde{\mathrm{a}}^{\dagger}$ the activation MFPT decreases with the intensity of the intrinsic noise, while for $\tilde{a} \gtrsim \tilde{a}^{\dagger}$, the activation MFPT increases with noise intensity. The activation MFPT lines for different volumes do not cross at the same point, and therefore the value of $\tilde{a}^{\dagger}$ cannot be defined precisely, but is approximately $\tilde{a}^{\dagger} \approx 3.2$ in the simple model and $\tilde{\mathrm{a}}^{\dagger} \approx 2.5$ in the detailed model. 


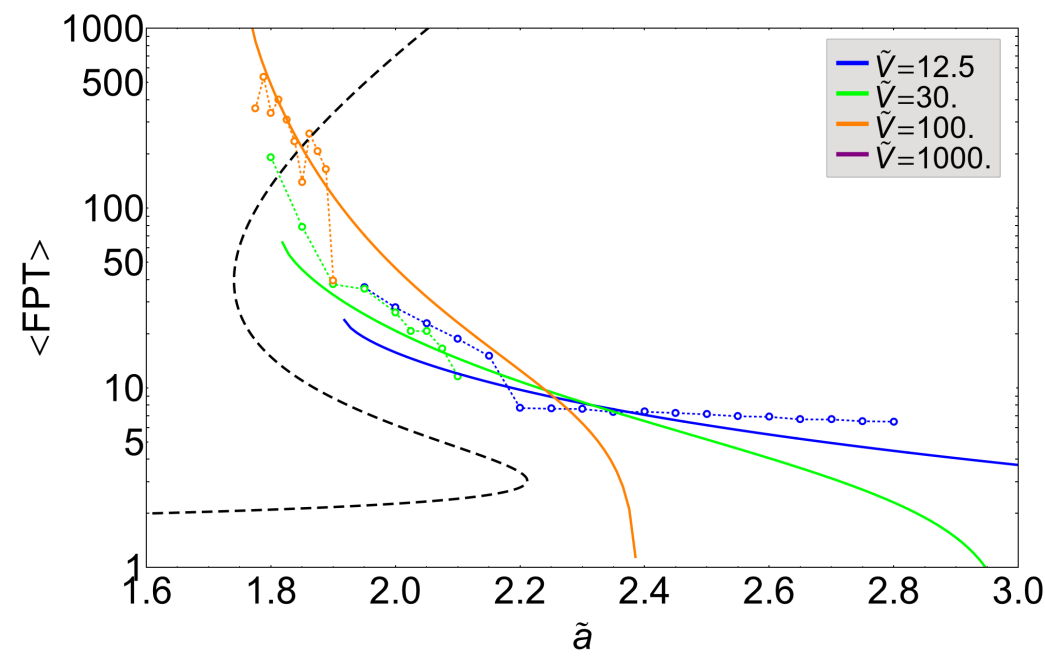

Figure 4.8: First passage time for the detailed model. Mean first passage time (MFPT) for the detailed model of genetic switch from the low stable state to the unstable state, Gillespie simulations (circles), for different volumes (see legend). The stable and unstable states are defined as the maximum and minimum of the steady-state distribution of the Gillespie simulations (see figure 4.6). The MFPT from the Fokker-Planck approach of the simple model (solid lines) are also reported for comparison. The deterministic bifurcation curve (dashed line) is depicted with an arbitrary y-scale in order to visualize the deterministic bistable region. The MFPT for the detailed model are similar to the results for the simple model, except that the bistable region is smaller. 

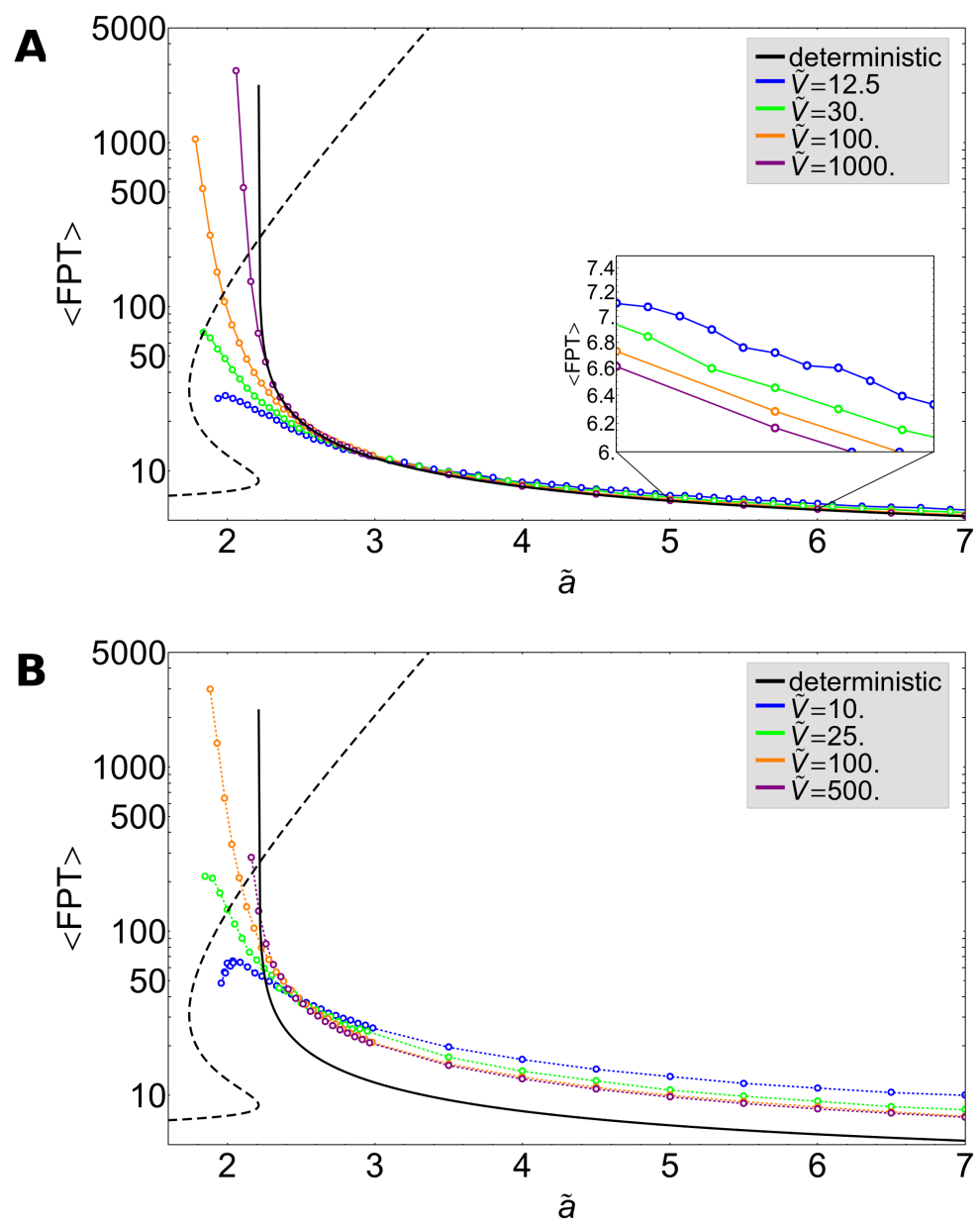

Figure 4.9: First passage time from low to high state for the simple and detailed models. Mean first passage time (MFPT) for the simple (A) and detailed (B) models of the genetic switch from the low stable state to a position close to the high state at $0.9 \tilde{x}_{\text {high, }}$ Gillespie simulations (circles), for different volumes (see legend). The low and high stable states are defined as the maxima of the steady-state distribution of the Fokker-Planck description. The deterministic bifurcation curve (dashed black line) is depicted with an arbitrary y-scale in order to visualize the deterministic bistable region. The FPT is also computed for the deterministic model outside the bistable region (solid black line). For both models, we observe that for high values of the control parameter a the MFPT increases with increasing noise intensity, while for small $\tilde{a}$ the MFPT decreases with noise. 


\subsection{DIsCUSSION}

The results of the detailed model differ quantitatively from the simple model when fluctuations are considered. In particular, the probability distributions and the range of values for which the latter are bimodal are different. Thus, although the deterministic descriptions of both models are totally equivalent (as long as the quasi-steady state approximation holds) this is not true when considering the biochemical fluctuations. We have found that the stochastic stabilization effects still holds in the detailed model, but that the extension of the range of bimodality is smaller than in the simple model. Similar differences have been found in other studies about genetic switches: for example in the case of the genetic toggle switch it has been shown that protein-protein interactions can be safely eliminated (adiabatically) but protein-DNA interactions, even though are also fast, lead to noticeable changes in the switching rates if neglected [118]. It is also interesting to place our findings in the context of the role played by different noisy sources, gene switching, translational and transcriptional, for defining the global attractor of bistable systems [79]. Thus, it has been recently shown that the modulation of the intensity of these fluctuations can actually condition the global attractor (the most represented phenotype) and that the elimination of gene switching noise by means of the adiabatic approximation can suppress some phenotypes. Our results are consistent with this study and provide further means to analyze and understand such phenomenology.

In this study, we have modulated the intensity of intrinsic fluctuations keeping the same concentration and varying the volume of the system. One may wonder how the number of proteins in our model and the intensity of the fluctuations compares with the situation found in real biological systems. The maximum intensity of noise we have used corresponds to a volume of $V \approx 2 \mu \mathrm{m}^{3}(\tilde{V} \approx 10)$, for which the average number of protein $X$ in the low state is $\sim 1$. Such volume is in fact the typical volume of an E. coli cell [187] and this very low copy number of proteins has been measured in bacteria. For example, the number of LacY repressors in the lactose operon of $E$. 
coli, an auto-activating genetic switch, has been found to be between $\mathrm{o}$ and 10, most of the cells having zero or very few molecules [36], and single molecule measurement of $\beta$-galactosidase in E. coli [30] have also reported an average level of $\sim 1$ enzyme per cell. Consequently, the number of molecules, and therefore the noise intensity, used in our study is consistent with experimental data on bacterial regulatory networks. Moreover, the intensity of intrinsic noise may be even larger when considering protein expression bursts [133], a phenomenon that we have not included in our modeling.

We have observed that the switching rate exhibits a complex behavior when varying the intensity of intrinsic noise. For values of the control parameter close to the deterministic bistable region, an increase in the noise intensity leads to a higher switching rate, while for larger values of the control parameter, we observe the opposite effect. This behavior is found in both the simple and the detailed models and the phenomenology is similar to the one described in the activation circuit of the QS bacteria in chapter 3. Our results indicate that the modification of the energy landscape due to the intrinsic noise is the main contribution to this behavior. While most of theoretical studies neglect this modification, it would be interesting to study the stochastic modification of the energy landscape in other biological switches. In the experimental study of Avendaño et al. [1o], the coupled positive and negative feedback loops of a modified galactose-uptake control system of Saccharomyces cerevisiae produce a bistable switch with tunable switching rate and range of bistability. The authors suggest that the negative feedback allows separate tuning of the switching rates, possibly by modulating the intrinsic fluctuations of the system. In such cases, we hypothetize that the modification of the energy landscape due to intrinsic noise could play a role in the behavior of the switching rate.

\subsection{CONCLUSIONS}

By using the auto-activating genetic switch as a case study, we have shown that the biochemical intrinsic noise may induce a shift in the 
position of the bifurcation points such that the region of parameter values for which the stationary probability distribution is bimodal increases with fluctuations with respect to the deterministic situation. In particular, the low state stability is extended; an effect that we call stochastic stabilization and that we have shown that, in essence, does not depend on the colored character of the fluctuations. The perturbative method that we have introduced is general and can be applied to any stochastic system describing a gene regulatory network. Yet, we point out that the method is limited to the case of one-dimensional stochastic differential equations for which the general solution of the stationary probability density can be written explicitly. Nonetheless, we have shown by means of simulations of a more detailed model, that the stochastic stabilization phenomenon does not depend on this particular detail thus suggesting a generic phenomenon in positive feedback switches (see also chapter 3). Previous studies $[162,203]$ have also found that noise changes the position and even the number of stable states [22]. In this regard, our study provides a theoretical framework to predict and understand such phenomenology. Whether our results are applicable or not to complex fate decision and differentiation processes is a matter of further research. In that regard, fate decisions in some embryonic stem cells are driven by an excitable dynamics that includes positive feedback loops as the one we have considered herein [83]. Hence, we speculate that noise would be also playing a role in redesigning the epigenetic landscape in those cases.

As we have seen, intrinsic noise can induce spontaneous transitions between the two stable states of the autoactivating switch. This is also true for other genetic switches such as the toggle switch [194] and has important implications in the design of new synthetic biology applications [141]. In the next chapter, we will study how the intrinsic noise in a biological system with bistability and cell-cell communication can lead to a phase transition similar to the Ising model in statistical physics. 



\subsection{INTRODUCTION}

Coordinated behavior in a population of cells is one of the fundamental process in nature, from bacteria which optimize their resources by activating costly genetic programs only in favorable conditions, to eukaryotes which coordinate groups of embryonic cells to form well-structured differentiated tissues during development. Collective cell differentiation can be driven by environmental signals like morphogens [151] which form a gradient of concentration by diffusing through a tissue. In this case, cells are exposed to different signal levels depending on their distance from the source of morphogens which leads to region-specific cell differentiation. However, coordinated behavior has also been shown to arise solely from the interaction between cells, without the need of external signals, in a process similar to self-organization. At the basis of this phenomenon is cell-cell communication, which allows cells to coordinate and synchronize the expression of genes. During embryonic development, groups of precursor cells generated by an embryonic induction tend to stay together and maintain the expression of the same set of genes, even in the absence of external signal, a mechanism called the community effect $[25,157]$. In this case, cell-cell communication has been shown to play a crucial role in maintaining stable differentiation inside the territory, showing that the coordination of the cell in the group relies on the interaction between the cells. In prokaryotes, many synthetic and natural systems based on the QS communication exhibit coordinated behavior (see section 1.2). Synthetic genetic oscillators have been synchronized by QS signaling [41], leading to 
the emergence of autonomous gene expression oscillations at the level of a cell population similar to the circadian rhythms in higher organisms. By coupling cell density and cell motility by QS communication, E. coli cells have been shown to exhibit spatiotemporal patterns of cell density during the growth of a colony [104]. The formation of these patterns is solely due to the interaction between cells mediated by the QS signal and does not depend on any external signals.

All these processes must take place in the presence of fluctuations that unavoidably affect cellular functions. One central question is therefore how cells robustly coordinate in the presence of noise. This question has been studied in chapter 3 in the case of the activation of a positive-feedback switch by QS communication. In this chapter, I focus on the collective behavior of a population of toggle switches that are coupled by two QS signaling pathways and I show that the interplay between noise, bistability and cell coupling leads to a new mechanism of coordination at the level of the population. Depending on the diffusion rate, the cell population exhibits a disordered behavior or a totally coordinated behavior. The sudden transition in the degree of coordination in the cell population appears very similar to a second order phase transition. From the point of view of statistical physics, a phase transition is described as an abrupt change in the degree or type of order in the system. This change originates from cooperative phenomena due to the interaction between individual elements of the system. For example, attractive interaction between water molecules leads to transitions between the gas phase (vapor), the liquid phase and the solid phase (ice), depending on the temperature and the pressure. Phase transitions may also be a driven force in many biological processes. For example, lipids and proteins in the cell membrane segregate and form two-dimensional liquid rafts that are nanoscale assemblies that function in membrane signaling and trafficking [102]. Biophysical experiments on lipid membrane model systems suggest the coexistence of two liquid phases with different compositions of raft lipids [168, 102]. A key study on giant plasma membrane vesicles isolated from living cells has shown that the membrane organization exhibits a phase transition from one 
liquid phase at high temperature to two liquid phases at low temperature [192]. Typical behaviors of phase transition such as critical fluctuations have been observed in this system. Moreover, the proximity of the critical point temperature to the physiological temperature suggests that mammalian plasma membranes are tuned to reside near the miscibility critical point and that critical fluctuations might explain some of the heterogeneity of membrane composition. Other examples include the localization of $P$ granules during the asymmetric cell division in the Caenorhabditis elegans zygote, a phenomenon similar to liquid-gas phase transition $[97,27]$. While these examples describe transitions at the level of the molecular organization in the cell, much less studied are phenomena of phase transitions at the level of cell populations, induced by the interaction between cells in a tissue or in a bacterial colony. In this chapter I present a theoretical model of a population of bacteria that exhibits a phase transition. I will show that cell- cell coupling mediated by QS communication can lead to a transition from a disordered phase to an ordered phase with all the cells in the same state.

The toggle switch is one of the simplest genetic circuit exhibiting bistable behavior [56] and represents a minimal example of cellular decision making. Theoretical works have shown that cell-cell communication could coordinate a population of toggle switches [91]. In the experimental work of Kobayashi et al. [9o], the authors designed a toggle switch circuit interfaced with the QS signaling pathway of Vibrio fischeri. In addition to the toggle switch plasmid, cells also carried a plasmid where lacI was under the control of an autoinducer-dependent promoter. By adding exogenous autoinducer to the environment, the total expression level of LacI was temporarily increased, driving the switch from the low LacI state to the high LacI state. A similar transition was achieved by inserting the luxI gene into the plasmid and allowing a growing colony to trigger the transition in a cell density dependent manner. In this case, each cell would start producing the autoinducer during the colony expansion, initiate the QS activation, and produce the additional amount of LacI proteins that would flip the switch. However, these designs only allowed to trigger the transition into one direction, from the 
low LacI activity state to the high LacI activity state. In the following, I show that coupling by two parellel QS pathways leads to a different kind of transition at the level of the cell population.

\subsection{OBJECTIVES AND SUMMARIZED RESULTS}

In this chapter, I introduce a simplified model of a toggle switch interfaced by two different QS signaling pathways, such that the two stable states of the switch are coupled across the cell population. In this design, cells in the low LacI state can induce the low LacI state in the other cells, and cells in the high LacI state can induce the high LacI state. Therefore, each cell tends to induce the other cells in the colony to adopt the same state as its own, a phenomenon similar to the coupling between ferromagnetic spins. In the absence of QS signaling, cells behave like noisy switches, spontaneously jumping between the two stable states. I show that increasing the rate of diffusion (the coupling strength) leads to a phase transition from an unordered population to a globally coordinated population with all the cells in the same stable state, a transition similar to the second order phase transition in the mean-field Ising model. The existence of the phase transition is further supported by the presence of a peak in the susceptibility and by the computation of the critical exponents by finite size scaling. The same transition is found in a population of cells growing exponentially in a closed volume, with all the cells entering the ordered state upon reaching a critical system size.

In addition, the response of the cells to an external signal that introduces an asymmetry in the switch is studied. We find that for a diffusion rate below the critical point, cell-cell coupling enhances the sensitivity of the population response, while for a diffusion rate above the critical point, the response exhibits hysteresis, with the existence of two metastable states. Thus, the overall behavior of the system resembles the response of the ferromagnetic Ising model to an external magnetic field.

The change in the behavior of the cells arise from the interplay between fluctuations, the bistability of individual cells and cell-cell 
communication. This phenomenon of phase transition suggests a new mechanism for collective cell decision making and sets the theoretical basis for new applications as increasing the sensitivity and robustness of biosensors.

\section{$5 \cdot 3$ METHODS}

\subsubsection{Toggle switch}

The genetic toggle switch is composed of two genes, lacI and $\lambda c I$, that encode for two regulatory proteins, LacI and $\lambda \mathrm{CI}$, respectively [56]. Each gene is controlled by a promoter that is inhibited by the repressor that is transcribed by the opposite promoter. This design has been extensively studied, both experimentally $[56,8,90]$ and theoretically [196, 184, 194, 105], and has been shown to exhibit bistability over a wide range of parameter values. This system has two distinct stable states: one with a high expression level of lacI and low expression level of $\lambda c I$, and vice-versa. The dynamics of the toggle switch are usually described $[56,194]$ by the deterministic rate equations

$$
\begin{aligned}
& \dot{u}(t)=\alpha_{1}+\frac{\beta_{1} K_{1}^{3}}{K_{1}^{3}+v^{3}}-k_{d 1} u \\
& \dot{v}(t)=\alpha_{2}+\frac{\beta_{2} K_{2}^{3}}{K_{2}^{3}+u^{3}}-k_{d 2} v
\end{aligned}
$$

where $u$ and $v$ are the concentrations of repressor proteins $u$ (LacI) and $V(\lambda \mathrm{CI}), \alpha_{1}$ and $\alpha_{2}$ the basal rates of expression of the proteins $\mathrm{U}$ and $\mathrm{V}$ when the promoter is fully repressed, $\alpha_{1}+\beta_{1}$ and $\alpha_{2}+\beta_{2}$ the maximal expression rates in absence of repression, $k_{d 1}$ and $k_{d 2}$ the degradation rates, and $K_{1}$ and $K_{2}$ the concentrations of repressor proteins that lead to half maximum repression. The cooperativity in the repression of both regulatory proteins is reflected in the Hill coefficient, $n_{1}=n_{2}=3$, which leads to the bistable behavior.

Herein we only consider the symmetric case, where all parameters are equal for both proteins, i.e. $\alpha_{1}=\alpha_{2}=\alpha, \beta_{1}=\beta_{2}=\beta$, $\mathrm{K}_{1}=\mathrm{K}_{2}=\mathrm{K}$ and $\mathrm{k}_{\mathrm{d} 1}=\mathrm{k}_{\mathrm{d} 2}=\mathrm{k}_{\mathrm{d}}$. We set the time scale such 


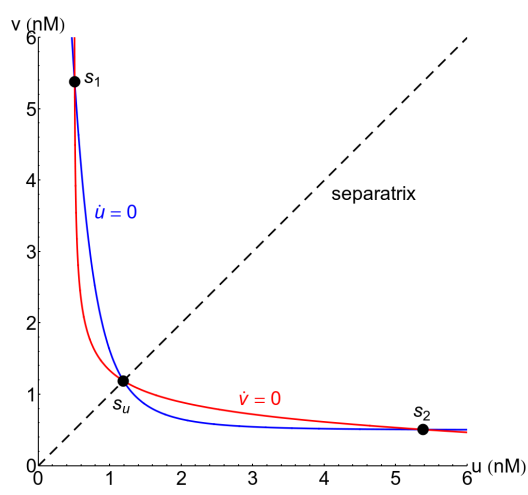

Figure 5.1: Phase plane of the symmetric toggle switch model. In the simple model of the symmetric toggle switch (equations (5.3) to (5.4)), the nullclines defined by $\dot{u}=0$ (red line) and $\dot{v}=0$ (blue line) intersect at three points: two stable states $\left(s_{1}\right.$ and $\left.s_{2}\right)$ and one unstable state $\left(s_{\mathfrak{u}}\right)$. Values of the parameters are $\alpha=0.5 \mathrm{nM}$, $\beta=10 \mathrm{nM}$ and $\mathrm{K}=0.5 \mathrm{nM}$.

that the degradation rate $k_{d}=1$ and in the following we write all the equations in dimensionless time units. We use $\mathrm{nM}$ units for the protein concentrations and rates of production, in order to compare them more easily to the number of molecules present in the cell, which influence the intensity of intrinsic fluctuations. The equations reduce to

$$
\begin{aligned}
& \dot{u}(t)=\alpha+\frac{\beta K^{3}}{K^{3}+v^{3}}-u \\
& \dot{v}(t)=\alpha+\frac{\beta K^{3}}{K^{3}+u^{3}}-v .
\end{aligned}
$$

By setting the parameters values to $\alpha=0.5 \mathrm{nM}, \beta=10 \mathrm{nM}$ and $\mathrm{K}=0.5 \mathrm{nM}$, we observe two stable states $s_{1}=\left(u_{s_{1}}, v_{s_{1}}\right)=$ $(0.508,5.38) \mathrm{nM}$ and $s_{2}=\left(u_{s_{2}}, v_{s_{2}}\right)=(5.38,0.508) \mathrm{nM}$, and one unstable state $s_{\mathfrak{u}}$, as shown by the intersections of the nullclines (see figure 5.1).

In the original experimental design [56], the toggle switch is robust to fluctuations and noise-induced transitions between the two 
stable states are extremely rare. In the experiments, the switch is usually flipped by using a transient signal that changes the activity of one of the two regulatory proteins [9o]. Spontaneous transitions, however, could also occur theoretically if the intensity of the noise is high enough. Stochastic simulations have shown that the rate of spontaneous transitions is inversely proportional to the average number of transcription factor molecules involved in the switch [196]. In our model, we choose the parameters set such that the number of molecules in the stable states is low enough to observe noisedriven transitions on a reasonable timescale. With the values of kinetic rates described above and a typical cell volume for an E. coli bacterium of $V_{\text {cell }}=1.5 \mu \mathrm{m}^{3}$, the average number of molecules in the two stable states of the toggle switch are 0.46 and 4.9 (stable states are symmetric in $u$ and $v$ ). We use the Gillespie algorithm [57] to simulate the stochastic dynamics of the reactions described by equations (5.3) to (5.4). The stochastic trajectory on figure 5.2 shows that the intrinsic noise drives spontaneous transitions between the high $u$ / low $v$ state to the low $u$ / high $v$ state. Such transitions might be observed experimentally in the toggle switch network by decreasing the expression rate of both proteins, for example by changing the ribosome binding sites. The behavior of individual cells is therefore determined by the random switching driven by noise. In the following, we will show that new behavior can arise at the level of a cell population when cells are coupled by means of chemical signaling (QS).

\subsubsection{Toggle switch interfaced by quorum sensing}

In order to study the collective behavior of a population of coupled genetic switches, we build a simplified model of a toggle switch interfaced by QS. We assume that the main regulatory proteins of the switch, $U$ and $V$, can diffuse into and out of the cell and act as QS signals. In the toggle switch, the LacI and $\lambda$ CI proteins cannot diffuse and an additional genetic module is needed to interface the switch with the QS signaling mechanism [90]. However, the QS 


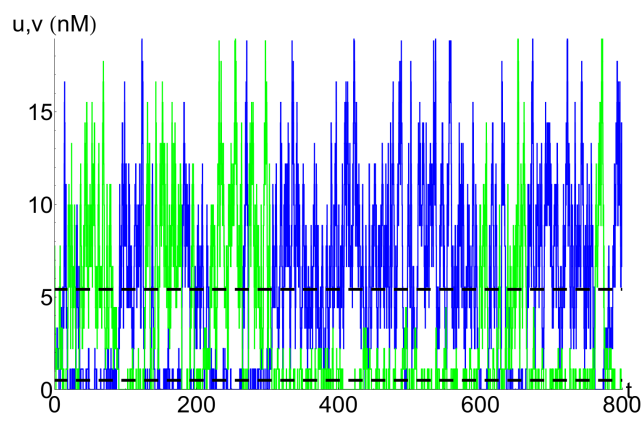

Figure 5.2: Noise-driven transitions in the individual toggle switch. Stochastic trajectory of an individual toggle switch system simulated using the Gillespie algorithm, concentrations of $u$ (blue line) and $v$ (green line). The switch undergoes spontaneous transitions between the two stable states, driven by the intrinsic noise due to the low number of molecules. Stable states of the deterministic model (dashed lines). Cell volume is $V_{\text {cell }}=1.5 \mu \mathrm{m}^{3}$. Time is in units of the protein lifetime $1 / k_{d}$.

module could be designed in such a way that the QS signal act at the same time as a reporter and as an inducer of the state of the switch (see section 5.5). In this case, a cell in the high $u$ / low $v$ state would produce and release the first QS signal, which would diffuse into the other cells and induce the production of $u$ on the receptor module, therefore promoting the high $u$ stable state. The same would be true for the second QS signal inducing the low $u$ / high $v$ state. We assume that we can simplify this genetic network by neglecting the details of the two QS modules and let the regulatory proteins $\mathrm{U}$ and $V$ act directly as QS signals. We use this simplified model as a first approach to study the behavior of a population of genetic switches coupled by two QS communication mechanisms. 
We consider a population of $\mathrm{N}$ identical cells, each carrying a copy of the same genetic network, communicating by the diffusion of molecules $\mathrm{U}$ and $\mathrm{V}$ (see figure 5.3). The reactions for each cell write

$$
\begin{gathered}
\varnothing \underset{1}{\stackrel{k_{u}}{\longrightarrow}} U_{i} \\
\varnothing \underset{1}{\stackrel{k_{v}}{\longrightarrow}} V_{i} \\
u_{i} \underset{r D}{\stackrel{\mathrm{D}}{\longrightarrow}} U_{e} \\
\mathrm{~V}_{i} \underset{\mathrm{rD}}{\stackrel{\mathrm{D}}{\longrightarrow}} \mathrm{V}_{e}
\end{gathered}
$$

with $k_{\mathfrak{u}}=\alpha+\frac{\beta K^{3}}{K^{3}+v_{i}^{3}}, k_{v}=\alpha+\frac{\beta K^{3}}{K^{3}+u_{i}^{3}}, D$ is the diffusion rate across the cell membrane, $r=V_{\text {cell }} / V_{\text {ext }}, U_{e}$ and $V_{e}$ the symbols for the molecules in the external volume and $i=1, \ldots, N$ the cell number. The rate equations for the whole population of cells write

$$
\begin{aligned}
& \dot{u}_{i}(t)=\alpha+\frac{\beta K^{3}}{K^{3}+v_{i}^{3}}-u_{i}+D\left(u_{e}-u_{i}\right) \text { for } i=1, \ldots, N \\
& \dot{v}_{i}(t)=\alpha+\frac{\beta K^{3}}{K^{3}+u_{i}^{3}}-v_{i}+D\left(v_{e}-v_{i}\right) \text { for } i=1, \ldots, N \\
& \dot{u}_{e}(t)=-u_{e}+r D \sum_{i=1}^{N}\left(u_{i}-u_{e}\right) \\
& \dot{v}_{e}(t)=-v_{e}+r D \sum_{i=1}^{N}\left(v_{i}-v_{e}\right) .
\end{aligned}
$$

It is clear that the diffusion of the molecules $\mathrm{U}$ and $\mathrm{V}$ offers a mechanism of global coupling between all the cells. A cell in the high $u$ state exports molecules of $U$ into the environment, which then enter into all the other cells and favor the high $u$ state. The same mechanism holds for the high $v$ state. The genetic switches tend to behave as ferromagnetic spins in the Ising model, interacting together to align their states. However, in opposition to the Ising model, the coupling between the cells is not restricted to the nearest neighbors. The external concentrations $u_{e}$ and $v_{e}$ act as two mean fields that couple 


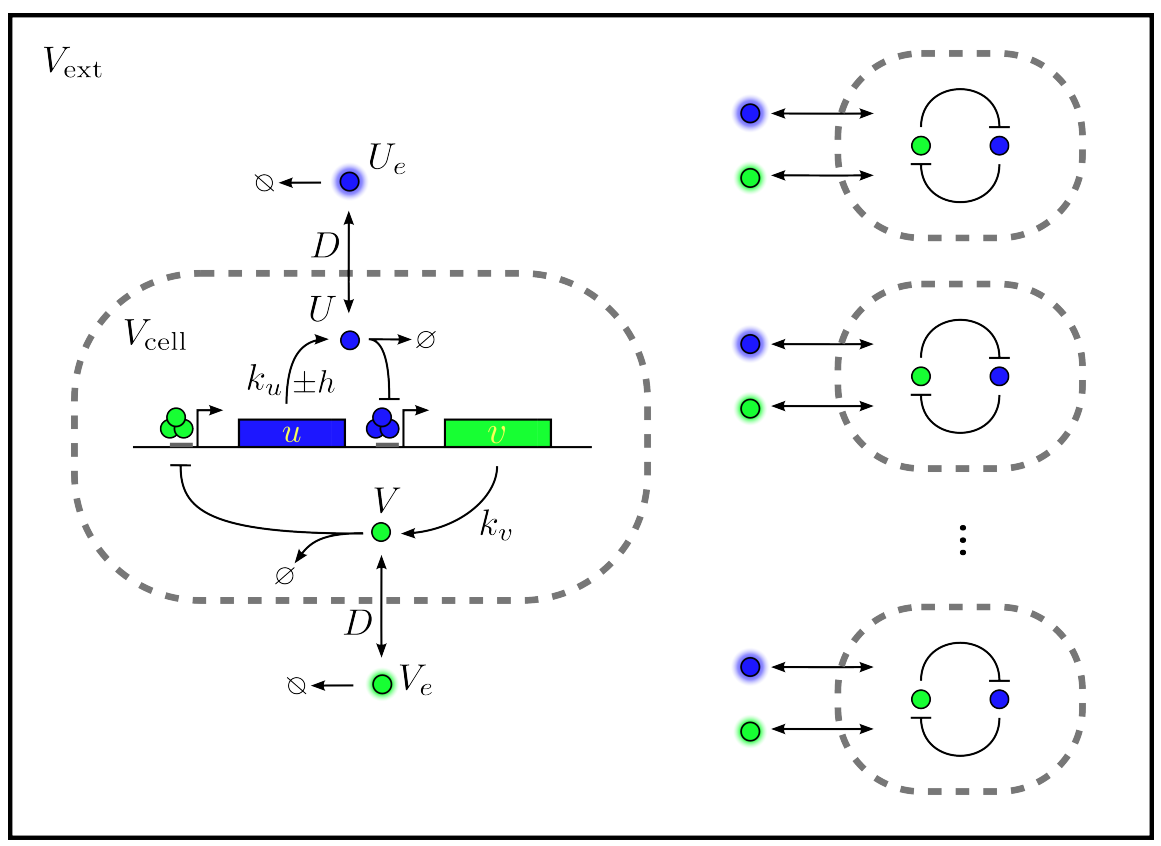

Figure 5.3: Scheme of the model of toggle switch interfaced by quorum sensing. The system is composed of $\mathrm{N}$ cells carrying the same symmetric toggle switch network. Expression of $U(V)$ is described by a Hill function $k_{u}(v)\left(k_{v}(u)\right)$ with cooperativity number $\mathrm{n}=3$. Proteins $\mathrm{U}$ and $\mathrm{V}$ act as signaling molecules by diffusing through the cell membrane with rate of diffusion D. A cell in the high $u(v)$ stable state produces and exports the signaling molecule $\mathrm{U}(\mathrm{V})$, which then enters into the other cells and promotes the high $u(v)$ state. The mechanism of diffusion of both signaling molecules leads to a system of cells with coupling similar to the mean-field ferromagnetic Ising model. Moreover, an external signal may introduce a bias in the switch by changing the basal rate of production of protein $U$ by an increment $h$ which can be positive or negative. 
all the cells together. This coupling is similar to the mean field approach in the Ising model in that each cell senses the same concentration of signaling molecules in the environment and is therefore subject to the same interaction.

As shown in the model of individual toggle switch, intrinsic noise drives stochastic transitions between the two stable states. The dynamics of an individual cell are therefore inherently random, constantly jumping between states $s_{1}$ and $s_{2}$. In this chapter, we seek to unveil how cell-cell communication can lead to global coordination in such a noisy system. In chapter 3, we studied the coordinated activation of a population of cells in which the QS signal, when reaching a threshold concentration, activated the regulatory circuit in all cells. In this chapter, however, the toggle switch is interfaced by two QS signals that drive the switch into opposite directions, such that the global outcome of a population of cells is not known a priori.

Motivated by the analogy with the Ising model, we explore the dynamics of a population of cells by varying the rate of diffusion while maintaining the same level of intrinsic noise. In the Ising model, a phase transition between an unordered state to an ordered state emerges when increasing the coupling strength (coupling coefficient J). This phase transition is similar to the one obtained when decreasing the temperature $T$ (see appendix A.2). In our model of toggle switch interfaced by QS, the strength of the cell-cell coupling is directly related to the rate of diffusion. In order to illustrate the role of the diffusion in the cell to cell coupling, we consider a system composed of one sender cell that produces $U$ at a constant rate $\alpha+\beta$ (maximum production rate of the toggle switch) and $\mathrm{N}-1$ receiver cells with no genetic circuit. The sender cell produces and exports $\mathrm{U}$ molecules into the environment, which enter the receiver cells. We report on figure 5.4 the concentration of $U$ in the receiver cells as a function of the diffusion rate $\mathrm{D}$. For $\mathrm{D}=0$, the signaling molecule cannot cross the cell membrane and there is no cell communication. At intermediate values of $\mathrm{D}$, only part of the signaling molecules produced by the sender cell diffuses into the environment and enters the receiver cells. The other part of the signaling molecules gets degraded in the environment. The faster the diffusion rate is, the 


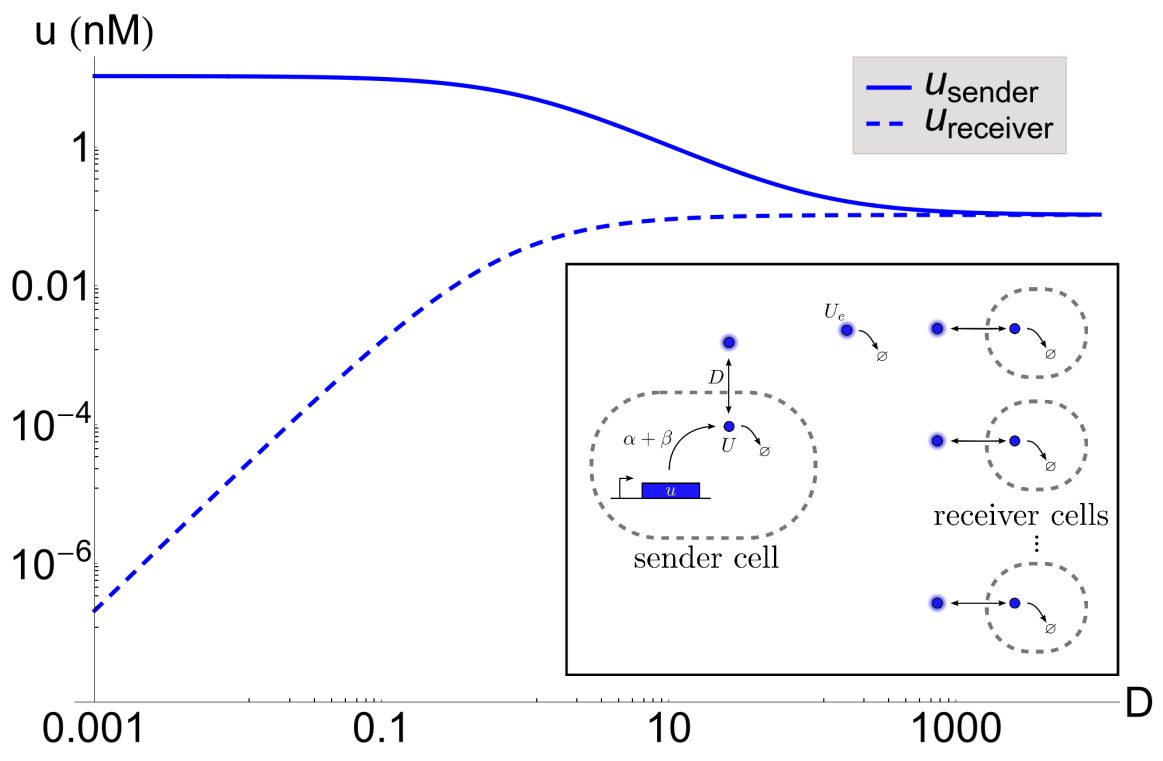

Figure 5.4: The level of signaling molecule reaching the cells increases with the diffusion rate. System composed of one sender cell that produces $\mathrm{U}$ at constant rate $\alpha+\beta$ (maximum production rate of the toggle switch) and $N-1$ cells with no genetic circuit (inset). When increasing the diffusion rate $\mathrm{D}$, the concentration of $\mathrm{U}$ in the receiver cells (dashed blue line) increases, while it decreases in the sender cell (solid blue line), due to the degradation of $\mathrm{U}$ in the environment and in the cells. For $\mathrm{D} \rightarrow+\infty$, the concentration of $u$ in the receiver cells reaches a maximum and equals the concentration in the sender cell. Increasing the rate of diffusion leads to a higher concentration of signal in the receiver cells and thus to a stronger coupling between cells. 
faster the signaling molecules reach the receiver cells, the less they get degraded during their travel. For $\mathrm{D} \rightarrow+\infty$, cell membranes can be disregarded and the system is equivalent to one unique volume containing all the cells. In this case, the concentration of $\mathrm{U}$ is the same in all the cells and the concentration in the receiver cell reaches its maximum. Therefore, in this simple case of one sender cell producing the signal, increasing the rate of diffusion leads to a higher concentration of signal in the receiver cells. The effect of the signal on the receiver cell will depend on the details of its genetic network. In the case of the toggle switch, we assume that a higher concentration of molecules $\mathrm{U}$ or $\mathrm{V}$ coming from the other cells will have an influence on the final state of the switch, either promoting the high $u$ state or the high $v$ state.

Notice that the size of the external volume also modifies the coupling strength, as a smaller external volume leads to a higher concentration of signaling molecules in the environment (data not shown). In the following, we choose a relatively small external volume in order to get a strong coupling between the cells and fix the ratio $\mathrm{Nr}=N V_{\text {cell }} / V_{\text {ext }}=1$. Thus, when changing the number of cells in the population, we keep constant the ratio between the total volume of the cells to the external volume. This value of the external volume corresponds to a very high cell density of $3.33 \cdot 10^{11}$ cells $/ \mathrm{ml}$. High cell densities has been observed in microfluidic traps of small colony size (about 1000 to $10^{\prime}$ ooo cells), reaching up to $2.2 \cdot 10^{11}$ cells $/ \mathrm{ml}$ [38].

We now consider a population of $\mathrm{N}$ cells carrying the toggle switch network and interfaced by two QS pathways. If the diffusion allows the signaling molecules to travel to the receiver cells and increase their concentration, it also removes molecules in the producer cell, leading to a lower steady-state concentration (see figure 5.4). The intensity of the intrinsic noise mainly depends on the number of molecules (see section 1.1). We seek to analyze the behavior of the system when the intensity of noise is constant (constant temperature) and the diffusion rate is varying (changing coupling coefficient). In order to keep approximately the same level of noise, we maintain constant the average level of the stable states $s_{1}=\left(u_{s_{1}}, v_{s_{1}}\right)$ and 
$s_{2}=\left(u_{s_{2}}, v_{s_{2}}\right)$ (see definition on page 144) of the switch by increasing the production rates $\alpha$ and $\beta$ when increasing the diffusion. We consider a population of $N$ cells that we assume are in the high $u$ state (see figure 5.5). At steady-state, the rate equations (5.9) to (5.12) reduce to

$$
\begin{aligned}
\dot{u}(t) & =\alpha+\frac{\beta K^{3}}{K^{3}+v^{3}}-u+D\left(u_{e}-u\right)=0 \\
\dot{v}(t) & =\alpha+\frac{\beta K^{3}}{K^{3}+u^{3}}-v+D\left(v_{e}-v\right)=0 \\
u_{e}(t) & =-u_{e}+\operatorname{NrD}\left(u-u_{e}\right)=0 \\
v_{e}(t) & =-v_{e}+\operatorname{NrD}\left(v-v_{e}\right)=0 .
\end{aligned}
$$

We fix the value of $u$ and $v$ to be the same in all the cells and equal to the steady-state $s_{1}=\left(u_{s_{1}}, v_{s_{1}}\right)$. With the value of the parameters described above, $\mathrm{K}=0.5 \mathrm{nM}$ and $\mathrm{Nr}=1$, we can solve numerically the equations for $\alpha$ and $\beta$ as a function of the diffusion rate

$$
\begin{aligned}
& \alpha=\frac{0.5+\mathrm{D}(1.5+\mathrm{D})}{(\mathrm{D}+1)^{2}} \\
& \beta=20-\frac{10}{1+\mathrm{D}} .
\end{aligned}
$$

In summary, we study the behavior of a system of $\mathrm{N}$ cells carrying the toggle switch network interfaced by QS described by reactions 5.5 to 5.8 by means of stochastic simulations using the Gillespie algorithm (see appendix A.I). We vary the diffusion rate D while maintaining the average level of the stable states of the switch by changing the production rates $\alpha$ and $\beta$, such that the intensity of intrinsic noise remains approximately constant. Increasing the diffusion rate leads to a stronger cell-to-cell coupling which may change the global behavior of the cell population. 


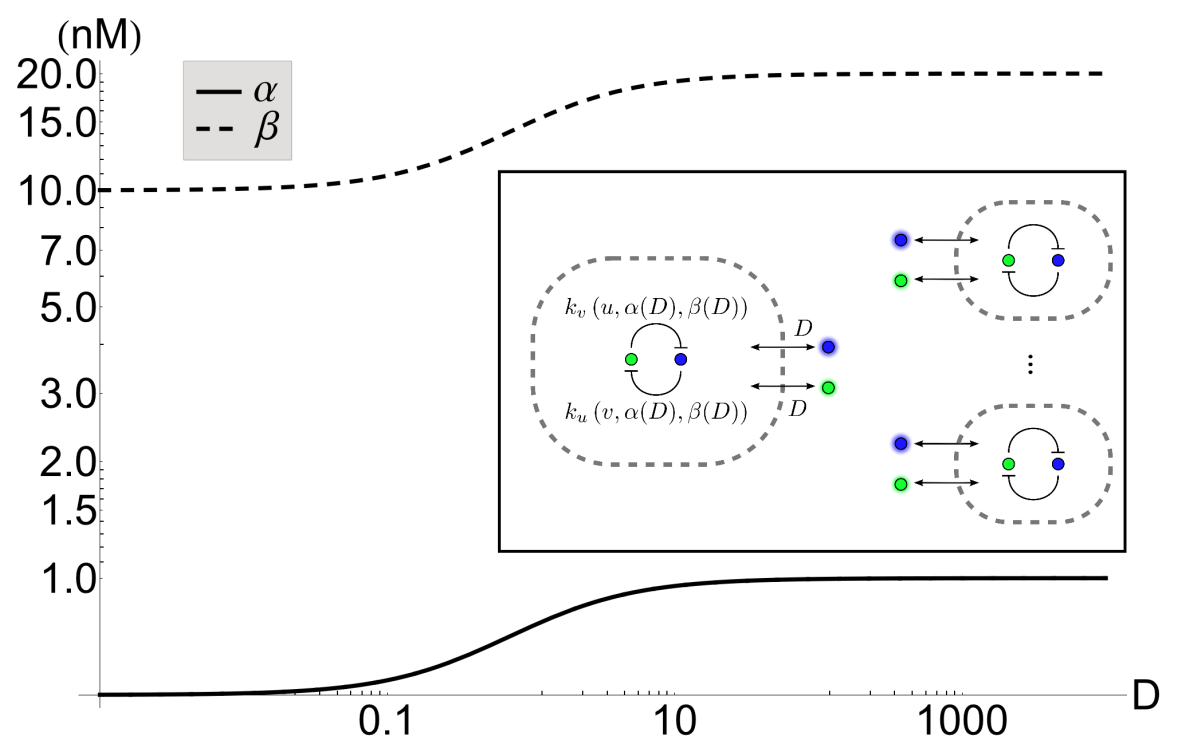

Figure 5.5: Tuning of production rates $\alpha$ and $\beta$ as a function of the diffusion rate to keep the average level of stable states constant. In the system of $\mathrm{N}$ toggle switches interfaced by QS (inset), we increase the production rates $\alpha$ and $\beta$ as a function of the diffusion rate $D$, such as to maintain constant the average level of the stable states of the switch and therefore the intrinsic noise. The functions $\alpha(D)$ (solid line) and $\beta(D)$ (dashed line) are derived by assuming that all the cells are in the high $u$ state and solving the rate equations (5.13) to (5.16) for $\alpha$ and $\beta$. Note that parameter $K$ is not changed. 


\subsubsection{Definition of observables}

In order to quantify the state of the system, we introduce the following definitions of observables. By analogy with the Ising model, the local order parameter is defined as

$$
S_{i}=\frac{u_{i}-v_{i}}{u_{s_{1}}-v_{s_{1}}} \approx \pm 1
$$

where $S_{i}$ is equal to +1 when the cell is in the stable state $s_{1}$ and -1 when the cell is in the stable state $s_{2}$ (the two stable states are symmetric). We define the magnetization

$$
M=\sum_{i=1}^{N} S_{i},
$$

the magnetization per cell,

$$
\bar{M}=\frac{1}{N} \sum_{i=1}^{N} S_{i},
$$

and the average magnetization per cell as

$$
\langle\bar{M}\rangle=\frac{1}{u_{1}-v_{1}}\left\langle\left\langle\frac{1}{N} \sum_{i=1}^{N} u_{i}-v_{i}\right\rangle_{\text {time }} \mid\right\rangle_{\text {traj }} .
$$

where $\langle\cdot\rangle_{\text {time }}$ is the average over time and $\langle\cdot\rangle_{\text {traj }}$ is the average over different trajectories. In the ordered phase, two possible fully ordered states can be achieved: either all the cells in the stable state $s_{1}$ (magnetization per cell +1 ) or all the cells in the stable state $s_{2}$ (magnetization per cell -1). The average magnetization per cell is the parameter measuring the order of the system (order parameter). Due to the symmetry of the system, we use the absolute value in the calculation of $\langle\bar{M}\rangle$ such that both the +1 and the -1 magnetization states, which are fully ordered, yield a positive order parameter of value +1 .

In the Ising Model, the susceptibility is defined as

$$
\chi_{\text {Ising }}=\frac{1}{N} \frac{\partial M}{\partial h}
$$


where $h$ is the external magnetic field, $N$ the size of the system and $M=\sum_{i=1}^{N} S_{i}$, the magnetization. This expression is evaluated at zero external field $h=0$. In numerical simulations, the susceptibility is usually computed as (using the fluctuation-dissipation theorem)

$$
\chi_{\text {Ising }}=\frac{1}{k_{B} T} \frac{1}{N}\left(\left\langle M^{2}\right\rangle-\langle M\rangle^{2}\right) .
$$

We define a similar susceptibility for the population of toggle switches interfaced by QS, assuming that the effective temperature remains constant,

$$
\chi=\frac{1}{N}\left(\left\langle M^{2}\right\rangle-\langle M\rangle^{2}\right) .
$$

\subsubsection{Critical behavior}

We define the reduced control parameter $\epsilon=-\left(D / D_{c}-1\right)$, which equals zero at the critical value of the control parameter $D_{c}$, is positive in the disordered phase and negative in the ordered phase. The finite-size scaling theory assumes that, for sufficiently large system sizes and for control parameter close to $D_{\mathfrak{c}}$, the following scaling relations hold,

$$
\begin{aligned}
\overline{\mathrm{M}}(\epsilon, \mathrm{L}) & =\mathrm{L}^{-\beta / v} \hat{\mathrm{M}}\left(\epsilon \mathrm{L}^{1 / v}\right) \\
\chi(\epsilon, \mathrm{L}) & =\mathrm{L}^{\gamma / v} \hat{\chi}\left(\epsilon \mathrm{L}^{1 / v}\right)
\end{aligned}
$$

where $\hat{M}$ and $\hat{\chi}$ are the rescaled magnetization per cell and susceptibility. The critical exponents of the phase transition are defined as

$$
\begin{aligned}
\bar{M} & \sim(-\epsilon)^{\beta} \quad \text { for } \epsilon<0 \text { (ordered phase) } \\
\chi & \sim|\epsilon|^{-\gamma} \\
\xi & \sim|\epsilon|^{-\gamma} .
\end{aligned}
$$

We first locate the critical order parameter $D_{c}$ by using the cumulant intersection method [21]. This method is useful for determining the location of the transition when the critical exponents are not 
known. We define the fourth-order cumulants, or Binder cumulants, as

$$
\mathrm{U}_{\mathrm{L}}=1-\frac{\left\langle\overline{\mathrm{M}}^{4}\right\rangle_{\mathrm{L}}}{3\left\langle\overline{\mathrm{M}}^{2}\right\rangle_{\mathrm{L}}^{2}}
$$

where $\langle\cdot\rangle_{\mathrm{L}}$ is the average value for a system size of $\mathrm{N}=\mathrm{L}$ cells. It can be shown that the Binder cumulant does not depend on the system size at the critical point. Moreover, as the system size goes to infinity, the Binder cumulants converge to the following universal values:

- In the symmetric phase, $\epsilon>0, \mathrm{U}_{\mathrm{L}}=0+\mathcal{O}(1 / \mathrm{L})$.

- In the ordered phase, $\epsilon<0, \mathrm{U}_{\mathrm{L}}=2 / 3+\mathcal{O}(1 / \mathrm{L})$.

- At the critical point, $\epsilon=0, \mathrm{U}_{\mathrm{L}}$ tends toward a universal fixed point $0<\mathrm{U}_{\mathrm{L}}^{*}<2 / 3$.

\section{$5 \cdot 4$ RESULTS}

5.4.1 The stochastic dynamics of the cell population exhibits an unordered phase for low diffusion rate and an ordered phase for high diffusion rate

As we have seen previously, the stochastic dynamics of the toggle switch in a single cell exhibits continuous jumps between the two stable states. In order to study the effect of the cell communication on the dynamics of the switches, we run stochastic simulations of a population of $\mathrm{N}=100$ cells for two different values of the diffusion rate, $\mathrm{D}=0.1$ and $\mathrm{D}=5$. Initial conditions are stable state $s_{1}$ for half of the cells in the population and stable state $s_{2}$ for the other half. The stochastic dynamics of all the individual cells in the population are plotted on figure 5.6. For $\mathrm{D}=0.1$, the individual cells exhibit uncorrelated dynamics similar to the isolated toggle switch, spontaneously jumping between the two stable states. The population is in an unordered phase, with no global coordination among cells. The external concentrations $\mathfrak{u}_{e}$ and $v_{e}$ are almost equal and fluctuate around their average level. This shows that the levels of 
signaling molecules $U_{e}$ and $V_{e}$ in the environment are equilibrated, with more or less half of the cells exporting $u$ molecules and the other half exporting $v$ molecules. The same behavior is observed for the population averages $\bar{u}$ and $\bar{v}$. For $\mathrm{D}=5$, we observe that in this simulation trajectory all the cells lock into the high $u$ state and fluctuate around this stable state, leading to an ordered phase. Individual cell transitions are very rare (data not shown). The concentration of $U$ in the environment and in the cells is much higher that the concentration of $\mathrm{V}$, showing that cells exchange $\mathrm{U}$ molecules and reinforce the high $u$ state. Thus, we observe two different behaviors depending on the rate of diffusion.

\subsubsection{The toggle switch interfaced by QS exhibits a phase transition when varying the diffusion rate}

We compute the stochastic dynamics of a population of toggle switches interfaced by QS for different values of the diffusion rate and the system size. For each set of parameters, we run 10 trajectories with simulation time $10^{4}$ to $5 \cdot 10^{4}$. The magnetization per cell and susceptibility are reported on figure 5.7. We clearly observe a secondorder phase transition where the system goes from an unordered phase for low diffusion rates with nearly zero magnetization (cells are randomly distributed between the two stable states) to an ordered phase for high diffusion rates with near unity magnetization (where all the cells are in the same stable state). The susceptibility presents a peak around the critical diffusion rate, a characteristic of second-order phase transitions. As the system size increases from $\mathrm{N}=10$ to $\mathrm{N}=1000$ the transition gets more abrupt and the peak in the susceptibility increases. Notice that the magnetization reaches a maximum value that is slightly higher than unity. This could be due to the fact that the position of the stable states in the stochastic system might differ from the position of the deterministic stable states $s_{1}$ and $s_{2}$, an effect that we have observed in chapter 4 .

In order to characterize better the phase transition, we use finitesize scaling theory [54] to compute the position of the critical point 
and the values of the critical exponents. Due to the finite system size of the numerical simulations, we do not observe the non-analytical behavior predicted by the theory of phase transitions. The discontinuity in the first derivative of the magnetization and the divergence in the susceptibility appear as a smooth change in the increase of $\bar{M}$ (resulting in a sigmoidal shape curve) and a rounded peak for $\chi$. As the system size increases, the apparent critical point shifts to the left, the transition in the magnetization becomes sharper and the peak in the susceptibility gets higher and narrower, resembling more and more the situation of an infinite system.

We compute the Binder cumulants for all the simulations and plot it as a function of the control parameter D (see figure 5.8). We ob-

Figure 5.6 (following page): Stochastic dynamics of a population of toggle switch interfaced by quorum sensing for low and high diffusion rates. Stochastic dynamics of a population of $N=100$ cells carrying the toggle switch network interfaced by QS for diffusion rate $\mathrm{D}=0.1$ (left column) and $\mathrm{D}=5$ (right column). Time series of concentration $u$ for all the cells in the population (density plots A and $\mathrm{E}$ ), time series of $u$ (blue line) and $v$ (green line) with deterministic stable states (dashed lines) for cell $\#_{1}$ (B and F), time series for external concentrations $u_{e}$ (blue line) and $v_{e}$ (green line) ( $C$ and $G$ ) and time series for concentration averaged over the population of $u$ (blue line) and $v$ (green line) (D and H). Initial conditions are stable state $s_{1}$ for half of the cells in the population and stable state $s_{2}$ for the other half. For $\mathrm{D}=0.1$, the individual cells exhibit uncorrelated dynamics similar to the isolated toggle switch, spontaneously jumping between the two stable states, leading to an unordered phase. The external concentrations $u_{e}$ and $v_{e}$ are almost equal and fluctuate around the same average level (same for the average concentrations $\bar{u}$ and $\bar{v}$ ), showing equilibrated levels of signaling molecules $U_{e}$ and $V_{e}$. For $D=5$, all the cells lock into the high $u$ state and fluctuate around this stable state, leading to an ordered phase. External concentrations $u_{e}$ is much higher than $v_{e}$, showing that cells exchange $\mathrm{U}$ molecules and reinforce the high $u$ stable state in the whole population. Population average indicates that almost all the cells are in a the high $u$ state. 

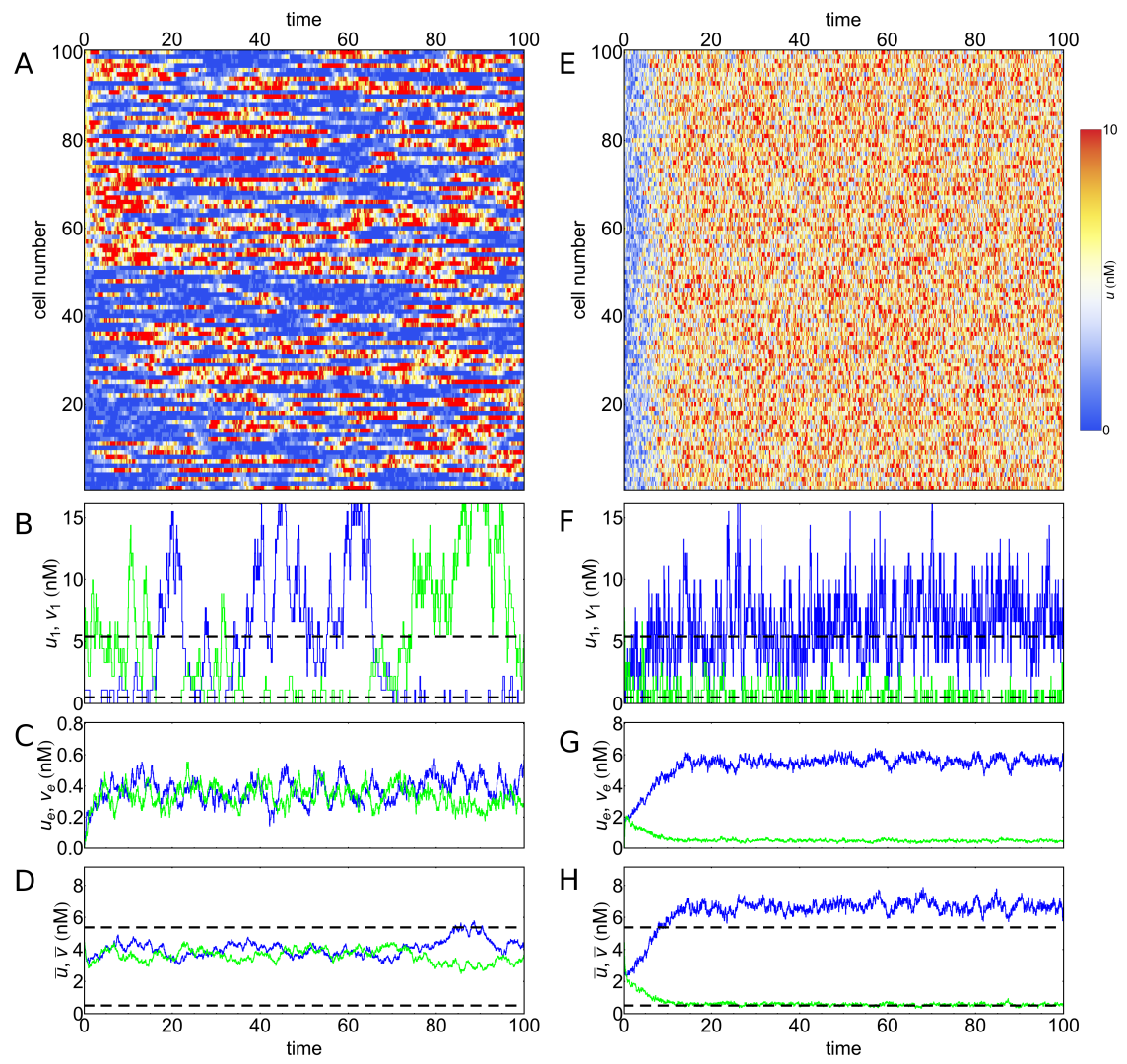

$\mathrm{H}$

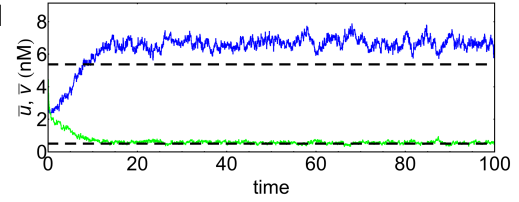


serve that all the $U_{L}(D)$ curves cross approximately at the same point, which is the critical point $D_{c}$. The intersection points of successive pairs of $U_{L}(D)$ curves for increasing system sizes $L$ (see inset in figure 5.8) converge to the critical fixed point. We use the intersection point of the two largest system sizes as the computed value of the critical point and obtain $D_{c}=0.797$.

As can be seen in figure 5.7, the apparent critical point or pseudocritical point shifts to the left when increasing the system size and converges to the critical point $D_{c}$ for infinite system. From the scaling relation (5.27), one can write that the position of the maximum in the susceptibility changes with the system size as

$$
\mathrm{D}_{\mathrm{c}}^{\chi}(\mathrm{L})=\mathrm{D}_{\mathrm{c}}+\mathrm{a}_{\chi} \mathrm{L}^{-1 / v}+\ldots
$$

Using the value of the $D_{c}$ we have found by the cumulants method, we fit the values of $D_{c}^{\chi}(L)$ (see figure $5.9 \mathrm{~A}$ ) to the expression above and find the critical exponent $v=1.70 \pm 1.15$ (the error is the standard error for parameter estimates from least square regression). Following the expression (5.27), we can also write a scaling relation for the height of the peak in the susceptibility,

$$
\chi_{\max }(\mathrm{L})=\mathrm{b}_{\chi} \mathrm{L}^{\gamma / \nu}+\ldots
$$

After fitting the values of the height of the peaks (see figure $5.9 \mathrm{~B}$ ) to this scaling relation and using the value of the exponent $v$ found above, we extract the critical exponent $\gamma=0.751 \pm 0.099$. Next, we use the scaling relation for the magnetization per cell at the pseudo critical point (see figure $5.9 \mathrm{C}$ ),

$$
\bar{M}\left(D_{\mathcal{c}}^{\chi}(L)\right)=c L^{-\beta / \nu},
$$

to fit the value of the critical exponent $\beta=0.060 \pm 0.396$, using the value of $v$ found above. We also plot on figure $5.9 \mathrm{E}$ the rescaled susceptibility $\hat{\chi}\left(\epsilon \mathrm{L}^{1 / \nu}\right)=\chi(\epsilon, \mathrm{L}) \mathrm{L}^{-\gamma / \nu}$ using the values of exponents $v$ and $\gamma$. The rescaled susceptibility for different system sizes $\mathrm{N}$ all collapse onto the same curve. The rescaled magnetization (figure 5.9D) defined in the scaling relation equation (5.26) also collapse onto the 


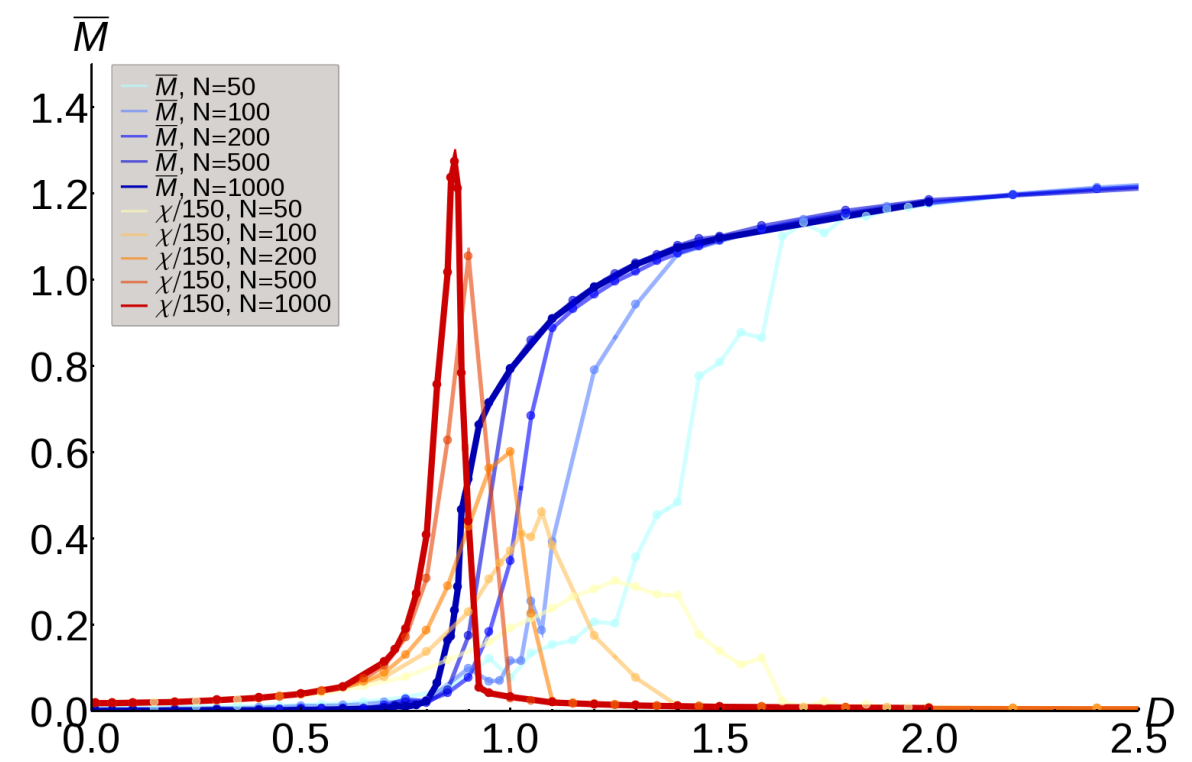

Figure 5.7: Phase transition in the toggle switch interfaced by QS system. Magnetization per cell $\bar{M}$ (cyan to blue color gradient lines) and susceptibility $\chi$ (yellow to red color gradient lines) for a population of toggle switches interfaced by QS as a function of diffusion rate $\mathrm{D}$. The susceptibility is rescaled by an arbitrary factor for ease of visualization. We observe a clear second-order phase transition from an unordered state with near zero magnetization (cells randomly distributed between the two stable states) to an ordered state with near unity magnetization (all the cells in the same stable state). The abruptness of the phase transition increases with system size, from $\mathrm{N}=50$ to $\mathrm{N}=1000$, as can be seen by the increase in the peak of the susceptibility and the slope of the magnetization curve. 


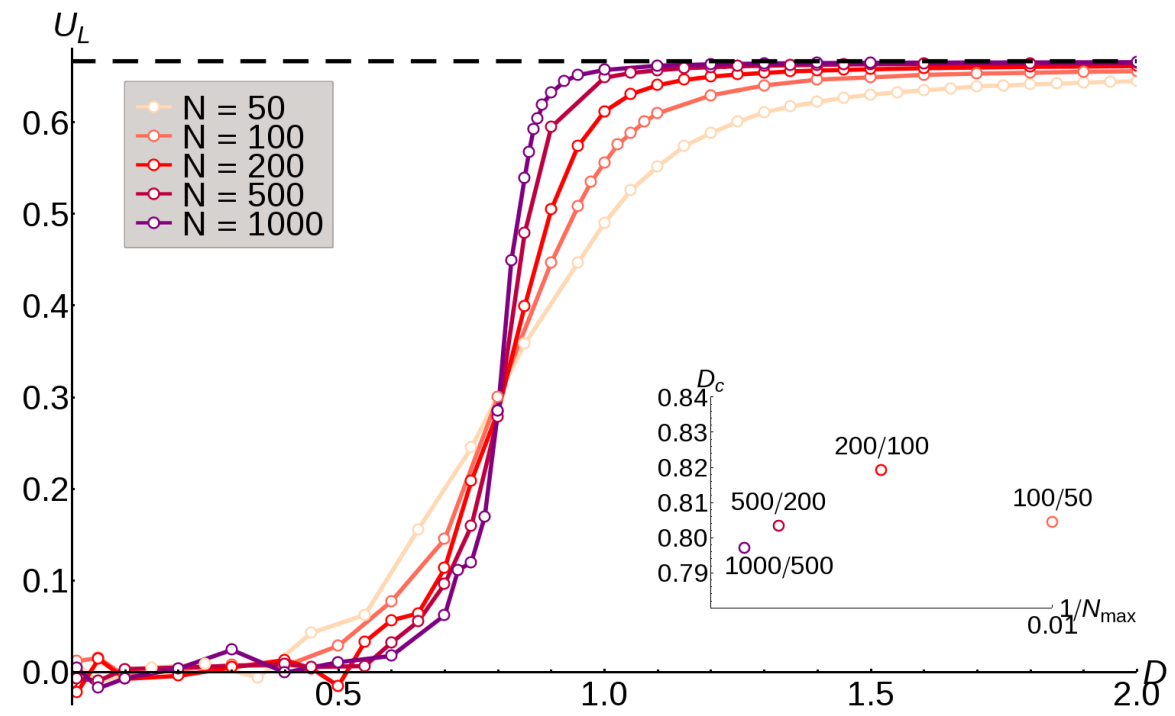

Figure 5.8: Critical control parameter estimate from intersection of Binder cumulants curves. Binder cumulant $\mathrm{U}_{\mathrm{L}}$ vs control parameter $\mathrm{D}$ (diffusion rate) for different system sizes $\mathrm{N}$ (orange to purple color gradient lines). In the finite-size scaling theory, the Binder cumulant reaches a fixed point $\mathrm{U}_{\mathrm{L}}^{*}$ at the critical value of the control parameter $D_{c}$. The fixed point does not depend on the system size neither on the critical exponents. The intersection of $\mathrm{U}_{\mathrm{L}}(\mathrm{D})$ curves for different system sizes gives the position of the critical control parameter. Intersection points for successively increasing pairs of system sizes L (inset) converge to the fixed point, $\mathrm{D}_{\mathrm{c}}=0.797$ for the pair $\mathrm{N}=1000 / \mathrm{N}=500$. 

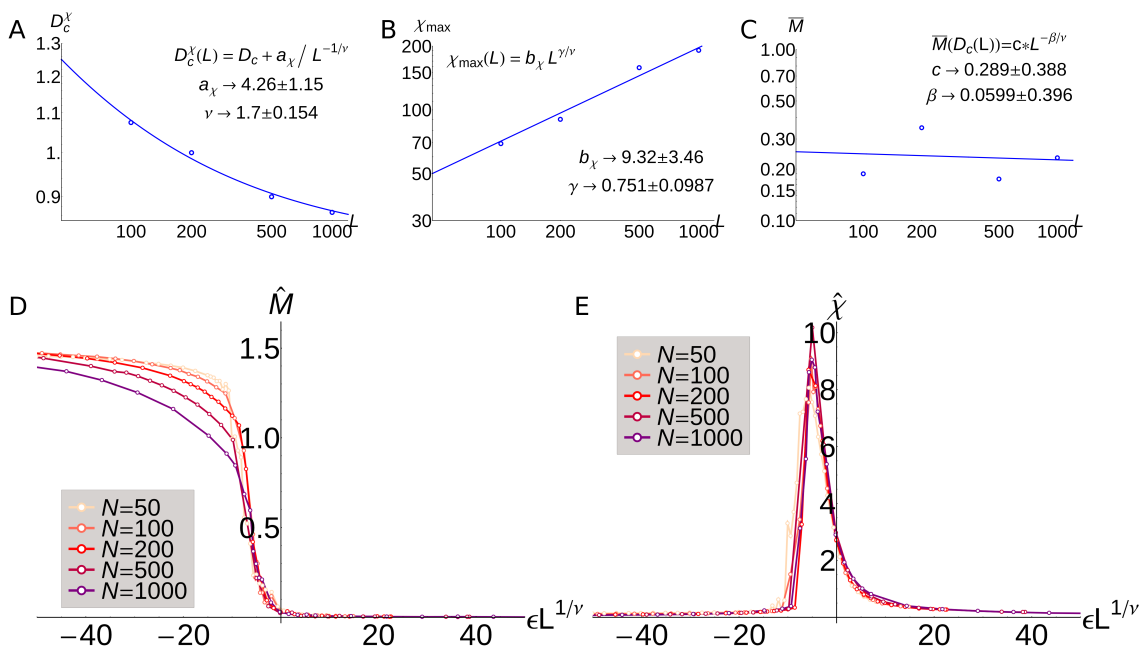

Figure 5.9: Fitting critical exponents using finite-size scaling of the susceptibility peak. (A) Position of the peak in the susceptibility $\mathrm{D}_{\mathrm{c}}^{X}$ as a function of system size L. A fit to the finite-size scaling relation $D_{c}^{\chi}(L)=D_{c}+a L^{-1 / v}$ gives the value of the critical exponent $v=1.70$. We use the value of $D_{c}$ found with the Binder $\mathrm{cu}-$ mulants method. (B) Height of the peak in susceptibility $\chi_{\max }$ as a function of the system size L. A fit to the finite-size scaling relation $\chi_{\max }(\mathrm{L})=b L^{\gamma / v}$ gives the value of critical exponent $\gamma=0.751$. (C) Magnetization per cell $\bar{M}$ at the finite-size critical point $D_{\mathcal{C}}^{X}(L)$ as a function of the system size L. A fit to the finite-size scaling relation $\bar{M}\left(D_{c}^{X}(L)\right)=c L^{-\beta / v}$ using the value of exponent $v$ yields the critical exponent $\beta=0.060$. (D) Rescaled magnetization $\hat{M}\left(\epsilon \mathrm{L}^{1 / \nu}\right)=\bar{M}(\epsilon, \mathrm{L}) \mathrm{L}^{\beta / \nu}$ using the values of exponents $v$ and $\beta$. (E) Rescaled susceptibility $\hat{\chi}\left(\right.$ epsilon $\left.\mathrm{L}^{1 / \nu}\right)=\chi(\epsilon, \mathrm{L}) \mathrm{L}^{-\gamma / \nu}$ using the values of exponents $\nu$ and $\gamma$. The rescaled susceptibility for different system sizes $N$ all collapse onto the same curve. 
same curve near the critical point. In summary, we found the following critical control parameter and critical exponents for the phase transition in the population of toggle switches interfaced by QS,

$$
\begin{aligned}
D_{c} & =0.797 \\
v & =1.70 \pm 1.15 \\
\gamma & =0.751 \pm 0.099 \\
\beta & =0.060 \pm 0.396
\end{aligned}
$$

Notice that the error on the critical exponent $\beta$ from the nonlinear regression are quite large and that the standard error calculated here does not take into account the propagated error from the estimated value of $v$, such that the final error for all critical exponent should be very large.

These critical exponents differ from the mean field theory of the Ising model when varying the coupling coefficient J (see appendix A.2):

$$
\begin{aligned}
& \gamma_{\text {M.F. Ising }}=1 / 2 \\
& \gamma_{\text {M.F. Ising }}=1 \\
& \beta_{\text {M.F. Ising }}=1 / 2
\end{aligned}
$$

We point out that while more data are needed in order to obtain precise estimates, the critical exponents in our model differ from the critical exponents of the Ising model, within the errors calculated above. Thus, our results suggest that the phase transition observed in the toggle switch interfaced by QS does not belong to the same university class as the mean field Ising model.

5.4.3 The toggle switch interfaced by QS exhibits a phase transition during exponential growth of a colony

In section 5.4.2, the global behavior of a population of toggle switches interfaced by QS has been shown to exhibit a phase transition when varying the diffusion rate. The population size $\mathrm{N}$ was fixed and stochastic simulations were performed for different values of the diffusion rate. In natural environments and in laboratory cultures, however, cells constantly grow and divide. In this section, we will show 
that a phase transition can occur in a population of proliferating cells in a closed volume. In this case, the phase transition is driven by two concurrent effects: the increase of the coupling strength due to the decrease in the external volume and the increase in the system size.

We run stochastic simulations of a population of cells carrying the toggle switch network interfaced by QS. The dynamics of the cell growth and division are the same as in section 3.3.2 and we use the Colony software (see appendix A.I) to perform the stochastic simulations using the Gillespie algorithm. Notice that for these simulations there is no external dilution protocol and we keep the total volume of the system constant and closed. We use the same parameter values as above, starting with one cell in an external volume of $V_{\text {ext }}=600 \mu \mathrm{m}^{3}$, with a diffusion rate $\mathrm{D}=2$ that is above the critical point and $\alpha$ and $\beta$ are kept constant during the whole simulation as determined by expressions (5.17) and (5.18). The deterministic part of the cell cycle is $\tau=50$ and the deterministic/stochastic weight coefficient $\lambda=0.8$ ( see expression (3.4)). The time evolution of the cells state is reported in figure 5.10A. The color code denotes the concentration of $u$ in each cell. We observe that during the population proliferation, cells randomly jumps between the high $u$ and the low $u$ states. However, upon reaching a critical colony size at $t \sim 349$ and $N \sim 144$, the majority of cells enter the high $u$ state. This cell coordination at the level of the population can be observed in the time evolution of the magnetization per cell $|\bar{M}|(t)$ (figure 5.10B). In the first part of the simulation (till $t \sim 349$ ), cells do not exhibit any synchronization and randomly jumps between the two stable states of the switch. At the very beginning of the simulation, the very small size of the system $(N \lesssim 10)$ leads to large fluctuations in the magnetization. When the number of cells increases, the instantaneous average of the magnetization remains close to zero, showing that the states of the cells are not coordinated. Upon reaching a critical system size at $t \sim 349$ and $N \sim 144$, the magnetization per cell increases to one, showing that cells enter a coordinated state. Notice that in this simulation run, cells end up in the high $u$ state, but repeating the simulation with different random numbers can lead to 
the a global low $u$ state (see figure 5.11), due to the symmetry of the toggle switch model.

In this case the phase transition is driven by two concurrent effects. First, the external volume decreases at the same rate that the number of cells increases (figure 5.10C). As mentioned on page 151, a smaller external volume leads to higher concentration of signaling molecules in the environment and to a stronger coupling between the cells. Therefore, even if the rate of diffusion is fixed, the coupling strength increases during the exponential growth of the population. In the previous simulations, we have chosen a ratio of external volume of $\mathrm{Nr}=\mathrm{NV} \mathrm{V}_{\text {cell }} / \mathrm{V}_{\text {ext }}=1$, such that the sum of the volume of the cells is equal to the external volume. On figure 5.10C, we have plotted the evolution of the external volume $V_{\text {ext }}(t)$ and of the total volume of the cells $\sum_{i}^{N(t)} V_{i}(t)$. The intersection of these two lines shows

Figure 5.10 (following page): Phase transition in a growing population of toggle switches interfaced by QS leading to the high u state. (A) Time evolution of concentration $u$ in color code for an exponentially growing population of toggle switches interfaced by QS. D =2, cell cycle $\tau=50$, cell cycle deterministic/stochastic weight coefficient $\lambda=0.8, V_{\text {ext }}=600 \mu \mathrm{m}^{3}, \alpha$ and $\beta$ are kept constant. During exponential growth, cells randomly jump between the two stable states, upon reaching a critical population size at $t \sim 349$ and $N \sim 144$, beyond which the majority of cells lock into the high $u$ state. $|\bar{M}|(t)$ exhibits large fluctuations at the beginning of the simulation due to small system size, then remains close to zero in the unordered state and finally reaches unity when entering the ordered state. (C) External volume $V_{\text {ext }}(t)$ (solid line) and total volume of the cells $\sum_{i}^{N(t)} V_{i}(t)$ (dashed line). Cell coupling gets stronger when the external volume decreases, due to higher concentration of signaling molecules in the environment. Intersection of both lines indicates a ratio of cell volume to external volume $r$ equal to the previous simulation with fixed system size. Beyond this point the coupling strength is stronger and leads to the phase transition. The increase in the system size $\mathrm{N}$ with time enhances the phase transition. 

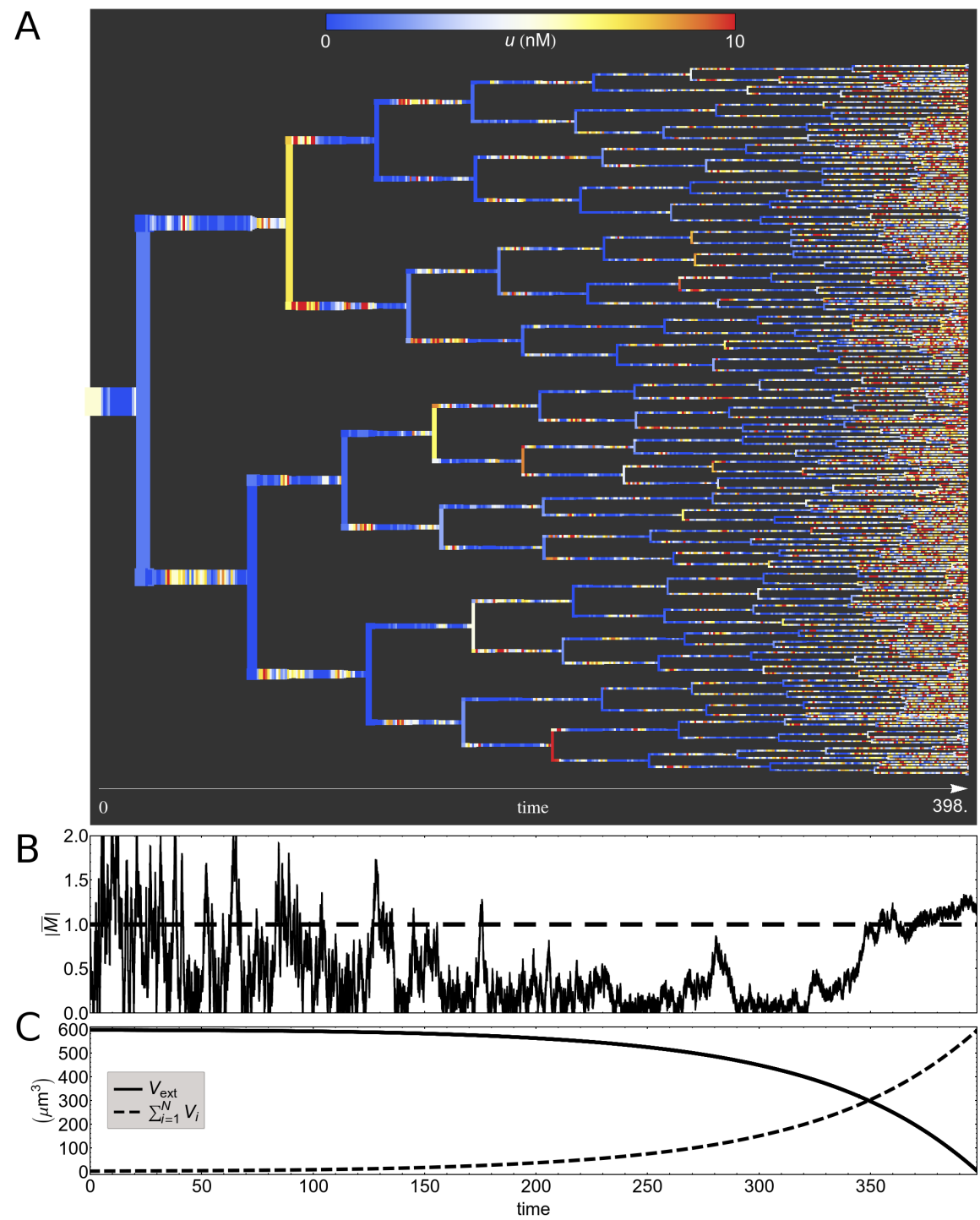
A

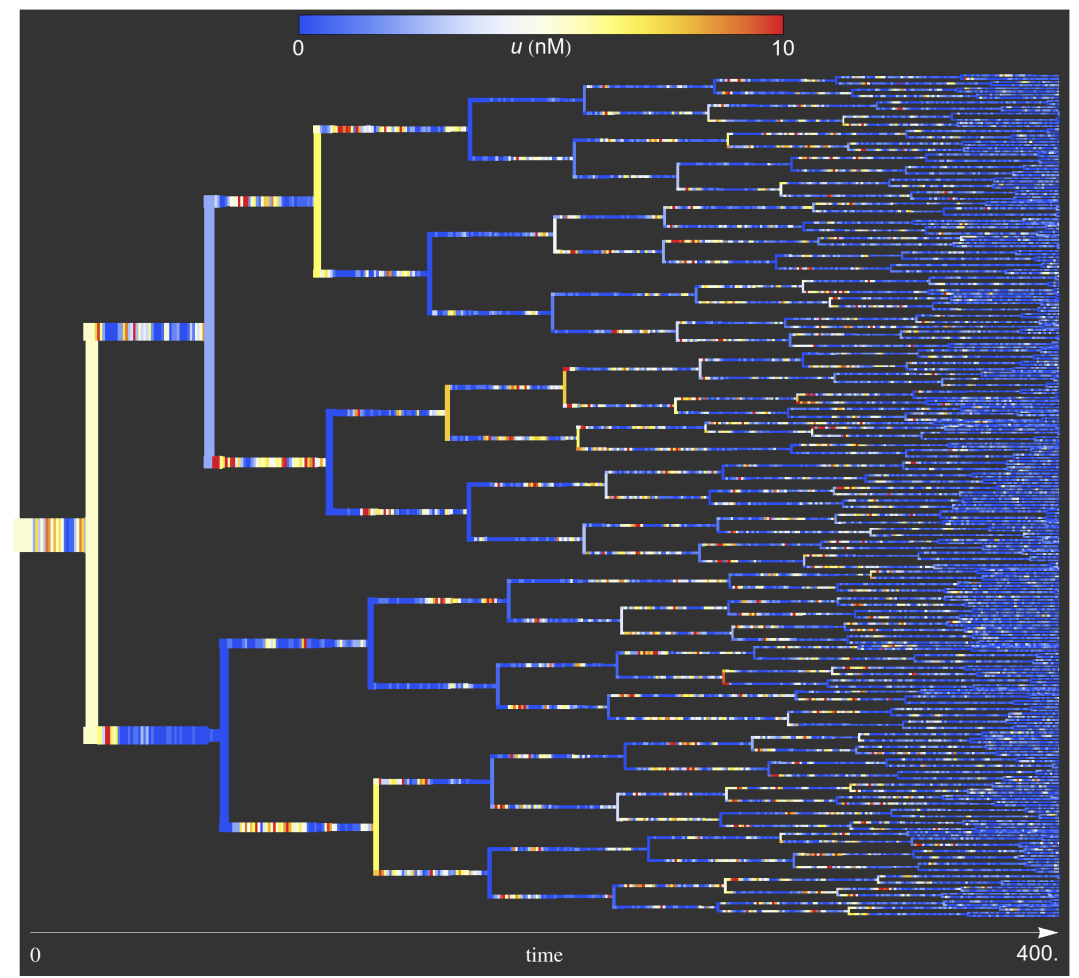

B

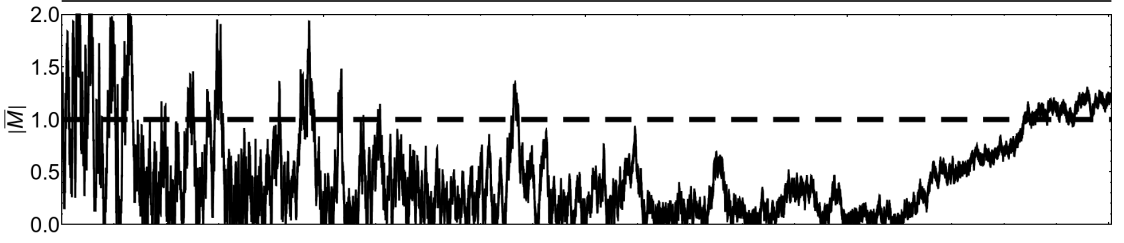

Figure 5.11: Phase transition in a growing population of toggle switches interfaced by QS leading to the low u state. Same plots as in figure 5.10 but for a simulation run with different random numbers, leading to an ordered state where all the cells are in the low $u$ state. (A) Time evolution of concentration $u$ in color code, indicating that all cells reach the low $u$ stable state (blue color). (B) At the end of the simulation the magnetization per cell $|\bar{M}|(t)$ reaches unity, showing the global coordination of the state of the cells in the population. 
that at $t=349$ the total volume of the cells is equal to the external volume and the ratio $r$ is the same as in the previous simulations of fixed-size population. For $t>349, r$ gets larger and leads to a stronger coupling strength. Second, the increase in the system size enhances the phase transition. As we have seen in the case of a fixed system size, the phase transition can only be observed for a system that is large enough. When the system size is very small, for example for $N=10$ (data not shown), the magnetization $|\bar{M}|$ increases very slowly with the diffusion rate D. When increasing the system size, the pseudo-critical point shifts to the left, towards smaller diffusion rates, and the transition in the magnetization becomes sharper. For the exponential growth simulation, we have chosen a diffusion rate $\mathrm{D}=2$ above the critical point. As the system size increases, the pseudo-critical point shifts to the left till crossing the fixed value of the diffusion rate. Therefore, we can expect the magnetization to increase when the system size increases till reaching the ordered state $(|\bar{M}| \sim 1)$ for a sufficiently large $N$.

\subsubsection{Cell-cell coupling enhances the population response to an external signal and leads to hyper-sensitive and hysteretic responses depend- ing on the diffusion rate}

The model of toggle switch interfaced by QS is perfectly symmetric, the parameter values being equal for proteins $\mathrm{U}$ and $\mathrm{V}$. As can be seen in the simulations for the population of fixed size (see figure 5.6), in the unordered phase cells have equal probability to be in the high $u$ state or in the low $u$ state. In the following, we analyze the response of the cells to an external signal that introduces an asymmetry in the switch. We assume that cells respond to the signal by changing the basal rate of production of protein $U$ to $\alpha+h$ (see figure 5.3). By analogy with the response of the spins to an external magnetic field in the Ising model, we expect the response of the cells to the external signal to change when the diffusion rate is either below, close to, or above the critical point. 
We run simulations of a population of a fixed size of $\mathrm{N}=1000$ cells (see figure 5.12). For $0 \leqslant t<t_{1}$, the external signal is switched off, $h=h_{0}=0$. At $t_{1}=500$, the external signal is switched on to a constant value $h=h_{1}$ and the dynamics are computed till $t_{\text {end }}=1000$. We compute the average magnetization $\langle\bar{M}\rangle$ of the cell response to the external signal for $t_{1}+50<t<t_{\text {end }}$. The response of the cells is computed for a range of external signals $h_{1}$ from -0.5

Figure 5.12 (following page): Response of a fixed-size population of toggle switches interfaced by $Q S$ to an external signal. The external signal changes the basal rate of protein $U$ production to $\alpha+h$, introducing a bias in the toggle switch circuit of all cells. (C) Average magnetization $\langle\bar{M}\rangle$ in a population of $N=1000$ cells coupled by QS in response to the external signal $h_{1}$, for diffusion rate $\mathrm{D}=0$ (black line), $\mathrm{D}=0.7$ below the critical point $\mathrm{D}_{\mathrm{c}}$ (orange line) and $\mathrm{D}=1$ above the critical point $\mathrm{D}_{\mathrm{c}}$ (red and purple lines). At $\mathrm{D}=0$ there is no cell communication and the response of the population is the average of single cell response. (D) From top to bottom, time series of external signal $h(t)$, concentration $u_{i}(t)$ in 100 sample cells in the population (see color code in figure 5.6), population-averaged concentrations $\bar{u}(t)$ (blue line) and $\bar{v}(t)$ (green line) and instantaneous magnetization $\bar{M}(t)$, at $D=0.7$ for $h_{1}=-0.2,-0.05,0,0.05,0.2$ (from left to right). The signal is introduced at $t=t_{1}$ at concentration $h_{1}$ and the response of the cells is computed for $t>t_{1}+50$. The population response is stronger and more sensible than the case $D=0$ due to the enhancing effect of the cellcell coupling. (A) Time series for $D=1$ and $h_{1}=-0.02,0.02$ (from left to right). The external signal is introduced at $t_{1}=0$. For $\mathrm{D}>\mathrm{D}_{\mathrm{c}}$, an very small external signal is enough to induce a large magnetization and the population response curve is discontinuous at $h_{1}=0$. (B) Time series for $D=1$ and varying $h_{1}$ from -0.5 to +1 and back to -0.5 (purple line in $(C)$ ). When varying the external signal the population get stuck into a metastable state of negative (positive) magnetization when increasing (decreasing) $h$ beyond zero, resulting in a hysteresis loop. 

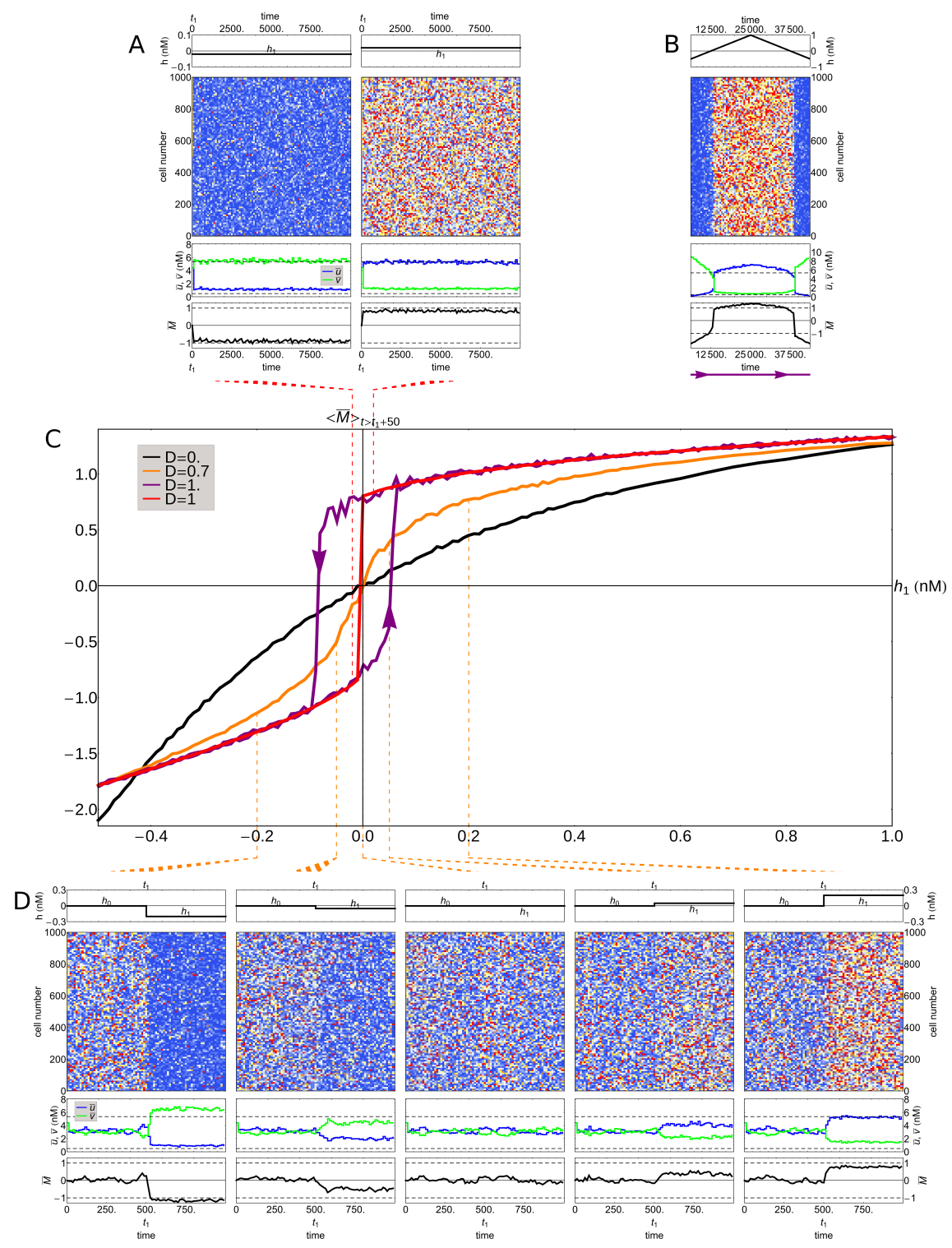
$\mathrm{nM}$ to $1 \mathrm{nM}$. In order to limit computation time, we run only one trajectory per parameter set.

For $\mathrm{D}=0$, there is no cell-cell communication and the response of the population is identical to the average of the response of a single cell. At $h_{1}=0$, the switches randomly jump between the two stable states and the average of the population magnetization (averaged over cells and averaged over time) is zero. When $h_{1}<0$ $\left(h_{1}>0\right)$, the external signal introduces a bias in the switch and the averaged magnetization becomes negative (positive). The resulting average magnetization exhibits a monotonous response curve to the external signal.

For $\mathrm{D}=0.7$ below the critical point, we observe the same qualitative behavior (see figure 5.12D). At $h_{1}=0$, cells randomly jump between the two stable states. As we have seen (see figure 5.7), the cell-cell coupling at $\mathrm{D}=0.7$ is not strong enough to produce a global cell response and the average magnetization is zero. For non-zero external signals, the average magnetization presents a sigmoidal shape that is much more sensitive than the diffusion-less case for small $h$. Cell-cell communication enhances the cells response to the external signal at the level of the population. The slope of the response curve for the diffusion-less case is $d \bar{M}_{D=0} / d_{1}\left(h_{1}=0\right)=2.23 \mathrm{nM}^{-1}$ while for $\mathrm{D}=0.7$ it is 4 times larger, $\mathrm{d} \bar{M}_{\mathrm{D}=0.7} / \mathrm{dh}_{1}\left(\mathrm{~h}_{1}=0\right)=$ $10.7 \mathrm{nM}^{-1}$.

For $\mathrm{D}=1$, above the critical point, all cells are stuck into the same stable state and present a finite magnetization even in the absence of external field. In the previous simulations of a fixed population size (see figure 5.7), we have computed the spontaneous magnetization that arises due to the cell-cell coupling. In the ordered phase at $\mathrm{D}=1$ and for $\mathrm{N}=1000$ cells, the absolute average magnetization was 0.79 . However, due to the symmetry of the toggle switch, the cell population ended either with a magnetization of approximately +0.8 or -0.8 , depending on the random numbers used for the computation of the trajectory. In this regime, the cell population is stuck into a metastable state and it is very difficult for the system to switch from the -1 to the +1 magnetization state and vice versa. Fluctuations in individual cells are not strong enough to 
overcome the cell-cell interactions that maintain the population in the ordered state. The lifetime of this metastable state is very large and surpasses the computational limits of our simulations. In order to minimize this effect and get a better estimate of the long-term response of the cell population to the external signal, we switch on the signal $h$ at the beginning of the simulation at $t_{1}=0$, such that the bias applies before the cell population has reached the metastable state. We observe (see figure $5.12 \mathrm{~A}$ ) that an external signal level as low as $0.01 \mathrm{nM}(-0.01 \mathrm{nM})$ is enough to drive the cell population to the metastable state with magnetization $\langle\bar{M}\rangle=0.82(\langle\bar{M}\rangle=-0.84)$. Therefore, as in the response of the spins to an external magnetic field in the thermodynamic limit of the Ising model, we observe a discontinuity in the average magnetization $\langle\bar{M}\rangle$ in response to the external signal $\mathrm{h}$.

It is well known that the response of a system of interacting spins to a varying external magnetic field can exhibit hysteresis. For $\mathrm{D}=1$, we compute the response of the cell population to an external signal varying linearly from $h(t=0)=-0.5$ to $h\left(t=10^{\prime} 000\right)=+1$ and back to $h\left(t=20^{\prime} 000\right)=-0.5$. The magnetization clearly exhibits a hysteresis loop (see figure 5.12, panel B and purple line in panel D), with the cell population's magnetization following a different trajectory for increasing $h$ than for decreasing $h$. The cell population remains in the metastable state of negative magnetization till $h \sim 0.05$ when increasing $h$ and remains in the metastable state of positive magnetization till $h \sim-0.08$ when decreasing $h$. Notice that this hysteresis phenomenon is not due to the bistability of the toggle switch in single cells, since in the case of zero diffusion, the magnetization is zero for $h=0$ and there is only one global attractor for every value of $h$.

\section{$5 \cdot 5$ DIsCUSSION}

In this chapter, we have presented a model of a toggle switch network in bacteria interfaced by QS such that cells tend to align the state of their switch. We have used a highly simplified topology for 
the network in order to contain the computational complexity and restrict the number of parameters. In the real toggle switch circuit, LacI and $\lambda \mathrm{cI}$ proteins cannot diffuse through the cell membrane or act as QS signals. However, the set of reactions 5.5 to 5.8 can be viewed as a first approximation to a more complex genetic network. In the following we suggest a model of a more realistic circuit that uses genetic components that have been successfully used in synthetic biology experiments.

The toggle switch circuit has already been interfaced by QS communication in the experimental work of Kobayashi et al. [90] using an additional communication module based on the LuxI/LuxR QS system. However, the coordinated transition of the population of switches was unidirectional, only allowing for the collective activation (high LacI state) of the cells. Herein we propose a design of genetic circuit composed of the toggle switch coupled to two QS communication modules (see figure 5.13). The two additional small genetic circuits act as communication modules using two different QS pathways. The first QS module produces a LuxI-type protein (LuxII) which synthetases the first QS signaling molecule (AII). The luxI1 gene is repressed by $\lambda$ cI protein. The first module also carries a biosensor of $\mathrm{AI} 1$ molecules and activates expression of a lacI gene copy in the presence of the autoinducer. Therefore, low levels of $\lambda \mathrm{cI}$ proteins in the cell activates the production of LacI proteins. When the toggle switch is in the low $\lambda \mathrm{cI} /$ high LacI state, the cell produces additional LacI proteins, reinforcing this stable state. However, because the autoinducer molecules can diffuse, the high LacI state is also reinforced in the other cells in the population. The second module works in the same way, by producing the second signaling molecule $\mathrm{AI}_{2}$ when the concentration of $\mathrm{LacI}$ is low, and producing $\lambda \mathrm{cI}$ proteins when detecting presence of AI2 molecules. In order to avoid crosstalks between the two signaling modules, two independent QS pathways are needed. Several QS modules have been used in synthetic biology applications, such as the LasI/LasR and RhlI/RhlR systems from Pseudomonias aeruginosa, which regulate biofilm formation, virulence, swarming motility and antibiotic efflux pumps [199]. LasI is the synthetase of the autoin- 


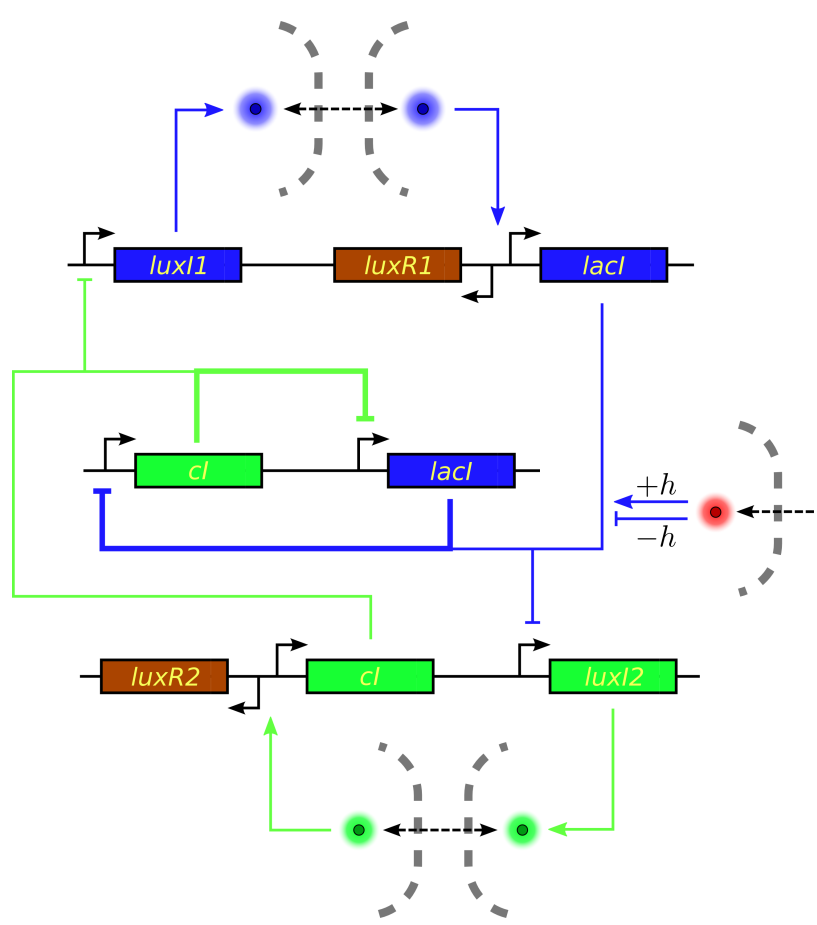

Figure 5.13: Scheme of a toggle switch circuit with two QS communication modules. Experimentally, the toggle switch network could be interfaced by two QS communication modules using different QS signaling molecules. The first QS module produces a LuxItype protein (LuxII) which synthetases the first QS signaling molecule (AI1). The luxI1 gene is repressed by $\lambda$ cI protein. The first module also carries a biosensor of AIı molecules and activates expression of a lacI gene copy in the presence of AI1. When the toggle switch is in the low $\lambda \mathrm{cI} /$ high LacI state, the cell produces AIr molecules which diffuses into the other cells and induce the low $\lambda \mathrm{cI} /$ high LacI state. The second module works in the same way, by producing the second signaling molecule $\mathrm{AI} 2$ when the concentration of LacI is low, and producing $\lambda \mathrm{cI}$ proteins when detecting presence of $\mathrm{AI} 2$ molecules. A response to an external signal could also be implemented by adding an externally controlled additional production of LacI protein (positive bias) or by introducing IPTG into the cells to partially inhibit LacI activity (negative bias). 
ducer N-(3-oxo-dodecanoyl)-homoserine lactone (30C12HSL) which is recognized by LasR. The other autoinducer synthetase, RhlI, produces $\mathrm{N}$-(butanoyl)-homoserine lactone $\left(\mathrm{C}_{4} \mathrm{HSL}\right)$ that activates its cognate receptor RhlR. These QS modules have been used in applications of synthetic biology, either individually by using one communication module [72] or using both modules working in conjunction. Communication systems have been created by combining the two QS systems, producing a consensus QS response dependent on the cell densities of two strains of E. coli [28], or creating a synthetic predator-prey ecosystem [11]. Notice that in these two experimental works, two different bacterial strains each carries a part of the genetic network, such that the genetic circuit introduced in each bacterium is relatively small. It would be probably more difficult to integrate the two communication modules together with the toggle switch in the same bacterial strain, due to the limitations of genetic engineering. However, the fact that the toggle switch has already been interfaced successfully with one QS module [90] suggests that it would be possible to design and implement a circuit with two QS modules. In addition, different ways to implement the response to an external signal could be envisioned. Any bias in the production or degradation rates of proteins $\mathrm{U}$ and $\mathrm{V}$ could in principle result in a population-level response. For example, one could change the promoter controlling transcription of gene $u$, resulting in a change in the repression strength. A positive bias in the production of protein $\mathrm{U}$ could be achieved by introducing a genetic module with the lacI gene under the control of a promoter that is activated by the external signal, producing an additional positive production of $\mathrm{U}$. A negative bias could be implemented by directly introducing IPTG into the environment, which enters the cells and inhibits the activity of LacI.

The phase transitions observed in the fixed population and growing population simulations arise from the interplay between the intrinsic fluctuations, the bistability of the toggle switch and the cellcell coupling. Models of bistable systems coupled by QS have been previously proposed but rely on only one coupling mechanism by the diffusion of one QS signal. For example, in the study of synthetic 
gene relaxator oscillators coupled by QS [91], the authors show that the intensity of noise is reduced when increasing the system size, leading to the restoration of bistability. For small system sizes, the cells are continuously jumping between two stable states. When the system size reaches a critical size, the population separates into two clusters of cells stuck into one of the two stable states. In this case, the response of the cell population is not fully coordinated but conserves some heterogeneity. The coupling by QS has also been proposed [194] to induce collective oscillations in a population of toggle switches when considering fluctuations in the degradation rates inside cells. As mentioned before, the synthetic circuit in [9o] leads to a unidirectional transition of a population of toggle switches, driving the activation of the high LacI state in all the cells, but not the deactivation. Our model, on the other hand, relies on two parallel coupling mechanisms mediated by two QS signals. The competition between the intrinsic fluctuations driving the switch flipping and the cell-cell coupling leads to a phase transition from an unordered phase to an ordered phase with all the cells stuck in the same stable state. This phase transition can be observed when increasing the diffusion rate or the system size and offers a novel mechanism of global and coordinated phenotypic change at the level of a cell population. The two parallel coupling mechanisms allow for two possible states in the ordered phase while maintaining full coordination in the population. Moreover, for diffusion rates below the critical point, the sensibility of the response of the cells to an external signal is enhanced while maintaining the magnetization to zero in the absence of signal. We believe that all of these features would not be possible to achieve with only one QS coupling mechanism.

The response of a population of cells to an external signal is greatly enhanced by the two parallel cell-cell coupling mechanisms. For a diffusion rate below the critical point, the slope of the response curve (average magnetization) for small signal concentrations is 4 times larger than in the case of zero diffusion. This increase in the sensitivity of the response is due to the noise-suppression effect of the cell-cell coupling [91] (see also chapter 2). In the presence of the external signal, the cells still randomly jump between the two sta- 
ble states, but the proportion of cells in the $+1(-1)$ magnetization state is higher when the signal is positive (negative). The cell-cell coupling reinforce the response by inducing more cells into the +1 $(-1)$ state. Thus, the response of the toggle switch interfaced by QS at the level of the population is higher compared to the toggle switch without QS, because the intercellular coupling increases the stability of the $+1(-1)$ state. Such a hypersensitive response could be used for the development of biosensors, overcoming the level of intrinsic noise present in small bistable genetic circuits. In this case, cell-cell coupling by QS could enhance greatly the measurement of the average fluorescence of a large cell population in response to an external signal, that would otherwise be diminished by the random switching of individual cells. For a diffusion rate above the critical point, the response of the population exhibits hysteresis when varying the external signal and recovers the subjacent bistable behavior of the single cells that was partially destroyed by noise. The strong cell to cell coupling leads to the existence of two metastable states of $\sim+1$ and $\sim-1$ magnetization with a switching time that increases with the system size. The combination of two parallel cell-to-cell coupling could therefore be useful for the design of memory units in synthetic biology applications to increase the robustness of the memory state at the level of the population.

We have shown that several parameters can control the phase transition in a population of toggle switches interfaced by QS: the diffusion rate, the system size and the external volume. The number of cells in the population really acts as the system size in the statistical physics meaning: increasing the number of cells increases the number of interacting constituents in the system and leads to a phase transition progressively resembling the one of the thermodynamic limit. On the other hand, the diffusion rate and the external volume both modify the characteristics of the communication mechanism and effectively change the coupling strength between the cells. Changing the diffusion rate experimentally would be probably difficult to achieve because the diffusion of the small autoinducer molecules is determined mainly by their size and the permeability of the cell membrane. However, any other parameter of the system 
that modifies the diffusion of signalling molecules could be used to control the phase transition. In many synthetic biology experiments, bacteria are cultured and imaged in microfluidic devices, allowing for well-controlled fluorescence imaging over long periods of time. In the experiment of Prindle et al. [140], QS-communicating bacteria are trapped in small chambers opened on one side on a channel where a continuous flow brings fresh nutrients and take away cells growing outside the chamber. The geometry of the chambers and the flow rates are key parameters that modulate the concentration of autoinducer inside the colonies. In this context, another way of changing the coupling strength could be to modulate the flow rate around the cell colonies, which can be easily achieved by changing the input pressure in the microfluidic channels.

\subsection{CONCLUSIONS}

Cell-cell communication can lead to the emergence of populationlevel behaviors that achieve important functions in biological systems and offers a range of new applications in synthetic biology [126]. In this chapter, I have presented a computational model of a population of toggle switches interfaced by two QS signaling pathways, such that a global coordination in the population appears when varying the control parameter of the system. We believe that this is the first model showing a global phenotypic change in a population of bacteria as a consequence of a phase transition in the statistical physics meaning. The change in cells behavior does not rely on any intrinsic properties of individual cells, but rather emerges from their interaction via QS signaling. We have shown that the emergence of global coordination requires a minimal system size and that the transition becomes more abrupt when increasing the number of cells.

The cell-cell communication has been modeled by allowing the two regulatory proteins of the toggle switch to diffuse through the cell membranes, such that the "on" state in one cell induces the "on" state in all the other cells; the same for the "off" state. We have 
shown that increasing the diffusion rate, which increases the coupling strength, leads to a phase transition from an unordered phase where the cells randomly jump between the two states of the switch, to an ordered phase with all the cells locked into one of the stable states. We have defined the magnetization per cell as the order parameter of the system, such that the magnetization is +1 when all the cells are in the "on" state, -1 when all the cells are in the "off" state, and 0 when cells are randomly distributed between the two states. The absolute value of the magnetization per cell exhibits the typical behavior of the order parameter in a second-order phase transition when varying the diffusion rate, remaining at zero till the critical value of the diffusion rate $D_{c}$ and then suddenly increasing. The existence of a phase transition is further supported by the presence of a peak in the susceptibility and by the computation of the critical exponents by finite size scaling. The same transition has been found in a population of cells growing exponentially in a closed volume, with all the cells entering the ordered state upon reaching a critical system size.

We have analyzed the response of the cells to an external signal that introduces an asymmetry in the switch. For a diffusion rate below the critical point, cell-cell coupling enhances the cell response to the external signal and increases the sensitivity. For a diffusion rate above the critical point, the cell population is always stuck in one of the two metastable states of +1 or -1 magnetization. In this case, the response to a varying external signal leads to hysteresis. We suggest that such a mechanism could be used to increase the robustness and sensitivity of biosensors.

In summary, we have shown that a population of toggle switches coupled by QS signaling undergoes a phase transition similar to the mean-field Ising model, when varying the coupling strength between cells. The change in the behavior of the cells arises from the interplay between fluctuations, bistability of individual cells and cell-cell communication. The coordinated state achieved for strong coupling is another example of noise reduction mechanism mediated by cell-cell signaling [91, 179] (see chapter 2). The phenomenon 
of phase transition suggests a new mechanism for collective cell decision making (see also section 6.1).

Herein we have assumed that the diffusion inside the external volume is fast and that the concentration of QS signals is homogeneous. We hypothesize that finite diffusion could lead to spatiotemporal patterning as the formation of clusters. Such pattern formation has already been observed in population of synchronized oscillators using QS signaling [41] and in wild-type V. fischeri [44]. However, we believe that for long time scales, the same phase transition should be observed in our model, since in the Ising model the main characteristics of the phase transition do not depend on the interaction length.

We have shown that the response of the cells to an external signal exhibits a ferromagnetic-like behavior. It would be interesting to study the response of a population of cells to an oscillating external signal. As shown theoretically [139] in the case of an ensemble of coupled noisy oscillators, the response of the system to a small periodic force shows a maximum at a certain system size. Such a system size resonance phenomenon also appears in the Ising model [139].

Another interesting effect is the interplay between the dynamics of a growing population and the pase transition. We have shown that a growing population can exhibit a phase transition to a fully coordinated state. Upon reaching a critical system size, the coupling between the cells is strong enough to overcome the intrinsic noise and lock the cells into the same state. It would be interesting to study the effects of cell growth rate and cell cycle on the behavior of the population. It has been shown experimentally in the case of a bistable genetic circuit [108] that the dynamics of cell proliferation can have a profound effect on the global state of the cell culture, due to the combined effects of stochastic switching and the logistic growth of bacteria. 

CONCLUSIONS AND PERSPECTIVES

\section{I SUMMARY OF ORIGINAL RESULTS}

In this thesis we have explored the roles of fluctuations and cell-cell communication in several gene regulatory networks in bacteria, at the level of the single cell and at the level of the cell population. We have focused on genetic switches as a paradigm of cellular decision making in both natural and synthetic bacterial systems. We have studied the effects of stochasticity in the emergence of collective behavior in populations of bacteria communicating by QS.

Our approach has been based on mathematical modeling and stochastic simulations, relying as much as possible on quantitative experimental data. We have used well-established deterministic methods, such as chemical rate equations, and stochastic methods, such as the Gillespie algorithm [57], together with analytical stochastic calculus, to describe the gene regulatory networks and the QS signaling mechanism. In order to study the collective behavior of bacteria, we have developed a computational software capable of simulating the stochastic dynamics of a population of cells communicating by diffusible signals that takes into account the cell growth and division (see appendix A.I). This computational tool is original in the sense that it combines stochastic dynamics of gene regulatory networks, cell-cell communication and cell proliferation dynamics. This software has been used throughout the thesis and has allowed us to analyze the effects of biochemical noise in the single cell and in the cell population.

Herein, we have applied our modeling approach to different biological systems. While in this thesis we have presented our research studies in the chronological order, in the following we describe the main results of each chapter from the perspective of model com- 
plexity. Thus, fluctuations affect the cell behavior at different levels, from a single isolated cell to a population of cells communicating by several QS signals. We have studied systems of increasing complexity: from an autoactivating genetic switch in an isolated cell (chapter 4), a population of cells with constitutive expression of a QS diffusible signal (chapter 2), a population of cells with the canonical LuxR/LuxI genetic switch communicating by QS (chapter 3), to a population of cells with a negative feedback switch interacting by the exchange of two different QS signals (chapter 5). In each of these systems, our study has contributed to a better understanding of the role of fluctuations in individual and collective cell behavior. In the following we summarize the main results of each chapter, in the order defined above. An illustrated overview of the main results of the thesis can also be found in figure 6.I.

In chapter 4, we have analyzed the effects of intrinsic noise in an autoactivating switch in an isolated single cell. While previous studies have shown that fluctuations can modify the epigenetic landscape of the genetic switch, there has been a lack of theoretical formalism to understand how those changes are driven by the biochemical fluctuations. We have developed a perturbative theory that shows that noise promotes the stability of the low-state phenotype of the switch and that the bistable region is extended when increasing the intensity of the fluctuations (see figure 6.1A), an effect that we have called stochastic stabilization. By performing stochastic simulations, we have demonstrated that this effect exists both in the simple model used in the analytical approach and in a more detailed model that takes into account the binding of the protein to the promoter, suggesting that the stochastic stabilization is a generic phenomenon in positive feedback switches. We have shown that increasing the intrinsic noise leads to a higher switching rate, but also modifies the energy barrier between the low and the high states, resulting in a complex behavior when varying the noise intensity. Our results indicate that the modification of the epigenetic landscape due to intrinsic noise not only modifies the steady states of the switch but also its switching dynamics, a phenomenon that is rarely taken into account in other theoretical studies. The results presented in chapter 4 
have shed light on the effects of intrinsic noise in the autoactivating genetic switch, by i) providing a theoretical approach to explain why and how the epigenetic landscape is modified due to biochemical fluctuations, ii) explaining the complex behavior of the stochastic switching when varying the intensity of noise. Thus, at the level of a single cell, intrinsic noise contributes to the cell-to-cell variability of the genetic switch and can modify its stable states and its dynamics.

In chapter 2, we have studied the interplay between intracellular noise and the diffusion process of the QS signaling mechanism. Previous studies have shown that diffusion can reduce the noise at the level of the signaling molecule. However, the role played by the different sources of stochasticity in the cell and their contribution to the dynamics of the autoinducer have not been characterized. We have built a model describing the expression of the signaling molecule and its diffusion in a population of cells. We have focused on the situation where the luxI gene is expressed at a low constitutive level, such that i) the feedback regulation of the luxI gene can be disregarded and ii) the transcription level is low enough to assume that at most one mRNA molecule can be present in the cell. Moreover, we have assumed that the production of the autoinducer from the mRNA can be described by a single reaction step. Based on these assumptions, we have been able to derive the analytical expression of the autoinducer distribution. By comparing the analytical results with stochastic simulations, we have determined the contributions of the transcriptional noise (mRNA copy number fluctuations) and the intrinsic noise in the fluctuations of the autoinducer. We have shown that varying the diffusion rate produces a repertoire of dynamics for the signaling molecule (see figure 6.1B). We have found that the transcriptional noise is the main contribution to the total noise for a large range of values of the diffusion rate. Moreover, we have observed that the total noise exhibits a maximum as a function of the diffusion rate, in contrast to previous studies. By observing the experimental values of diffusion rates in QS bacterial species, our results point toward the direction that bacteria have evolved to adapt their communication mechanism and avoid the diffusion rates that maximize the noise. In summary, in chapter 2 we have pre- 
sented a reduced model of gene expression and QS signaling that allowed us to i) reveal the contribution of intrinsic noise and transcriptional noise in the fluctuations of the signaling molecule, and ii) show that the competition of temporal scales between the mRNA dynamics and the diffusion process can lead to a variety of dynamics and fluctuations level for the autoinducer molecule. Thus, the QS communication mechanism modifies the fluctuations of the signaling molecule inside the cell and interacts with the gene expression noise.

In chapter 3, we have studied the effects of gene expression noise on the precision of the population coordination in the QS activation of the LuxR/LuxI system. The heterogeneity in the QS response observed experimentally in many bacterial species raises the question of how the precision of cells coordination depends on the intracellular noise sources. In order to answer this question, we have focused on one of the simplest QS regulatory network, the LuxR/LuxI system. By using both deterministic and stochastic models, we have analyzed the response and dynamics of a population of cells to different levels of autoinducer. We have shown that the activation of the QS switch near the critical concentration of autoinducer is very slow compared to the cell cycle duration, and, as a consequence, the non-stationary effects are important during the QS transition. Our results have shown that gene expression noise in LuxR is the main factor that controls the transient variability of the QS activation ( see figure 6.1C): increasing the burst size in LuxR decreases the precision of the QS activation. These results, together with experimental evidences on LuxR regulation in wild-type species, suggest that bacteria have evolved mechanisms to regulate the intensity of noise in LuxR and control the variability in the QS activation. Our study has shed light on the relation between the single cell stochastic dynamics and the collective behavior in a population of communicating cells.

In chapter 5 , we have studied a new mechanism of coordination in a model of toggle switch interfaced by two QS signaling pathways. Application of the QS signaling mechanism in synthetic biology has led to the creation of genetic circuits that display a variety of collective behaviors. We have presented a computational model of a 
population of toggle switches communicating by the exchange of two diffusible QS signals. The key feature of our model resides in the coupling of both the "on" and the "off" states between the cells. We have shown that increasing the diffusion rate, which increases the coupling strength between the cells, leads to a phase transition from an unordered phase where the cells randomly flip between the two states of the switch, to an ordered phase with all the cells locked into the same stable state (see figure 6.1D). The existence of the phase transition has been further supported by the presence of a peak in the susceptibility and by the computation of the critical exponents by finite size scaling. The same transition has been found in a population of cells growing exponentially in a closed volume, with all the cells entering the ordered state upon reaching a critical system size. Moreover, we have analyzed the response of the cells to an external signal that introduces an asymmetry in the switch and we have shown that the cell response exhibits a hysteresis loop, similar to the response of the ferromagnetic Ising model to an external magnetic field. We have found that for a diffusion rate below the critical point, the cell-cell coupling enhances the sensitivity of the population response and we have suggested that this new mechanism could be used to increase the robustness and sensitivity of biosensors. Our results suggest a new mechanism for collective cell decision making based on the phenomenon of phase transition.

From a broader point of view, the results presented in this thesis have shown that collective cellular decision making can be achieved by means of cell-cell communication, despite the unavoidable fluctuations in the individual cells. Our study has contributed to a better understanding of how cells in a population coordinate their behavior by exchanging small diffusible signaling molecules. We have shown that, when the diffusion is large, the QS mechanism effectively reduces the noise in the signaling molecule by averaging the fluctuations over the population and increasing the effective degradation rate of the autoinducer. This noise reduction mechanism enhances the cell coordination, as reported in previous studies. However, we have also shown that the interplay between intracellular noise and QS communication is more complex than first thought. Our results 
have shown that while the QS mechanism can reduce the fluctuations in the signaling molecule and lead to population-level coordination, it cannot suppress totally the effects of noise. The QS diffusion process only acts on the signaling molecule, and other sources of noise, such as gene expression noise, affect the reliability of the cell coordination. Thus, we have shown that both the time scale and the fluctuations in gene expression interfere with the communication process and lead to variability in the activation of the genetic switch. In the case of the LuxI/LuxR canonical system, the gene expression noise in LuxR reduces the precision of the cell coordination. Our results have shed light on the structure of some natural QS systems. In order to control the variability of the QS activation, cells may use additional regulatory mechanisms to control the fluctuations of the main components of its QS network, as for example negative feedback loops for LuxR. The effects of stochasticity in the mechanism of QS coordination can therefore be seen as a competition between the intracellular fluctuations, which drive the stochastic switching of the cells and produce variability, and the cell-cell communication, which reduces the noise and synchronizes cells in the population. In addition, we have shown that, when coupling a genetic switch by two QS signaling pathways that drive the switch into opposite directions, global coordination in the population can be achieved. The coordination in this system emerges when the coupling between the cells gets stronger than the intracellular noise, in a phenomenon similar to a phase transition. When the diffusion rate is small, the coupling between cells is weak and the intracellular noise dominates. When the diffusion rate is large, the coupling is strong and the cell-cell communication locks the cells into the same state. Therefore, we have taken advantage of the interplay between intrinsic noise and QS diffusion to design a system that exhibits a phase transition. We have shown that this transition from a disordered phase to a fully ordered phase could be used by bacteria to achieve robust collective cellular decision making in a growing population. 
A
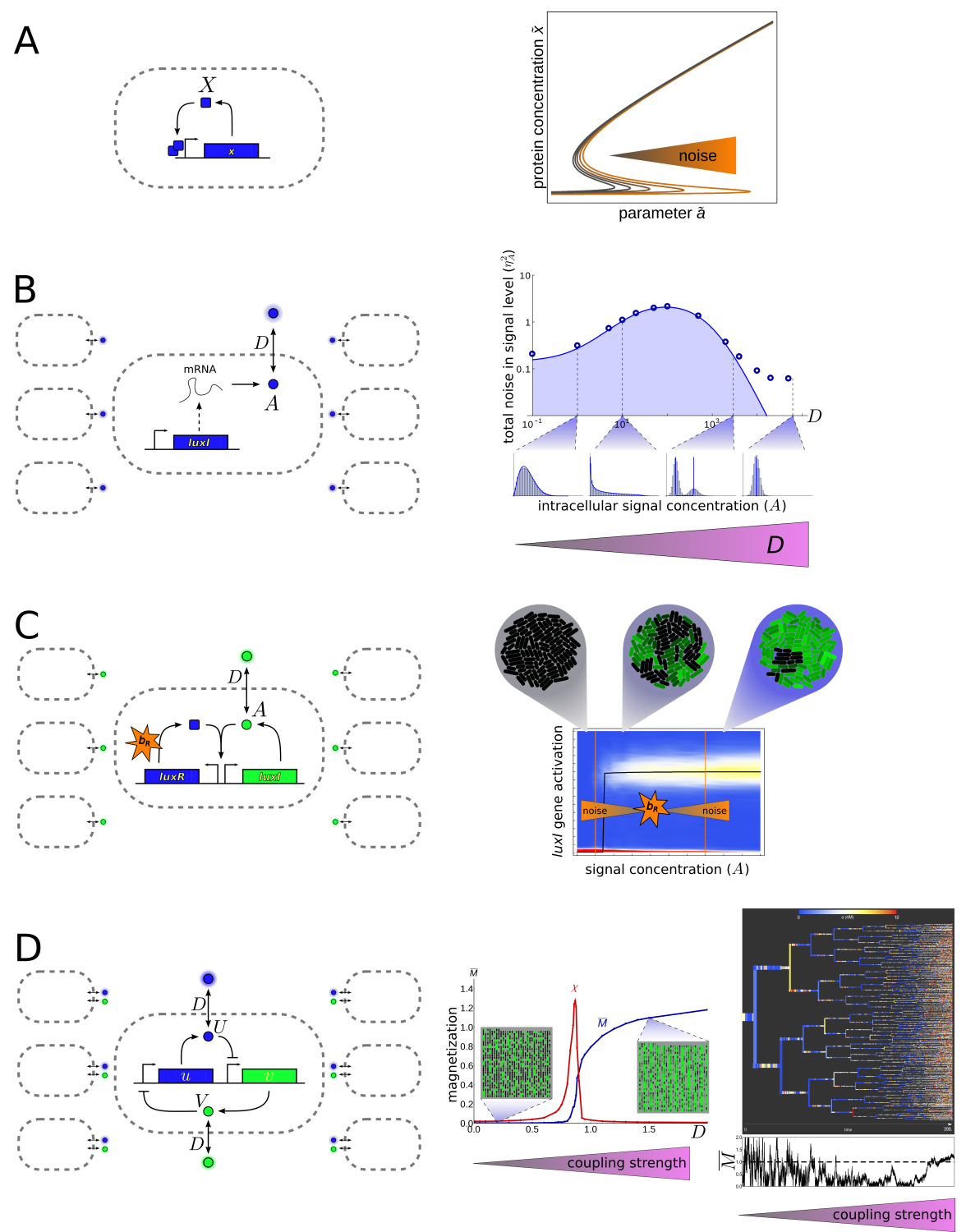

Figure 6.1: Illustrated overview of the main results of the thesis. The main results of each chapter are illustrated, from the simpler model to the more complex model. See main text for details. (A) Stochastic stabilization of phenotypic states: the genetic bistable switch as a case study (chapter 4). (B) Noise regulation by quorum sensing in low mRNA copy number systems (chapter 2). (C) Dynamics of the quorum sensing switch: stochastic and nonstationary effects (chapter 3). (D) Phase transition in the toggle switch coupled by quorum sensing: an Ising model in bacteria (chapter 5). 


\subsection{PERSPECTIVES}

The results presented in this thesis have opened a series of new questions. On whats follows, I present some of these questions and discuss on their implications both in the fields of mathematical modeling and biology.

In this thesis I have focused on the role of intrinsic noise in cells communicating by QS. While biochemical noise is one of the main sources of fluctuations in the cell, other sources of noise can influence cell functioning. In the context of our modeling approach, external fluctuations could also have important effects on the behavior of QS systems. Cell-to-cell variations in the rate of diffusion or in the rate of protein production could induce additional fluctuations in the level of molecules in the cell. Moreover, spatial variations of the concentration of autoinducer in the environment would result in different levels of signaling molecules sensed by each cell. While theoretical studies [71, 116] have suggested that the QS coordination mechanism is robust to such variations, further research is needed in order to understand the effects of external fluctuations in QS systems.

Many interesting aspects of the QS mechanism could be further studied and included in our modeling approach. Spatial effects as the finite diffusion of the autoinducer into the medium, can produce spatiotemporal patterns of activation of the QS genes in a population of bacteria. This phenomenon has been studied experimentally and theoretically. Signal diffusion in a narrow agar lane filled with QS reporter strain showed spatial and temporal patterns that differ significantly from the simple diffusion [44]. Dilanji et al. showed by mathematical modeling that the non-linearity of bacterial growth and of transcription activation lead to enhanced synchrony over the length scale of one centimeter. Diffusion of the autoinducer has also been shown to produce traveling waves of gene expression in a population of genetic oscillators coupled by QS [41]. How these coordinated behaviors remain robust to intra-cellular and extra-cellular noise sources remains a open field of study. 
Stochastic effects are expected to be especially relevant when the number of cells is very small. Groups of two or three Pseudomonas aeruginosa bacteria have been shown to initiate QS activation when confined in sub-picoliter volumes [24]. The confinement in a very small volume allows for the accumulation of the autoinducer which can reach the threshold concentration even with a few cells. In such conditions, the heterogeneity of the QS activation is even higher than in liquid cultures and fluctuations in the number of autoinducer molecules in the environment, in addition to the intra-cellular noise, could play a role in the variability of the activation. Our simulation software could be useful to study such systems, as it already takes into account the stochasticity in the levels of autoinducer in the environment. Moreover, confinement within small volumes could be highly relevant for studying wild-type bacterial species that often live in small numbers and in enclosed spaces, as in early stages of infection, biofilm formation or in soils [24, 33, 65].

The results presented in our study could be useful for Synthetic Biology approaches that exploit the QS mechanism. The fact that some important features of the QS mechanism, e.g. precision, rely on the burst size of one component, opens the door to modifications of the LuxI/LuxR operon for regulating the response depending on the problem under consideration. For example, the transcription rate of luxR could be increased and its translational rate decreased, which would lead to an higher precision of the QS activation.

Our results on the phase transition in chapter 5 suggest a new possible mechanism for cellular decision making in development. The global coordination between cells appears upon reaching a critical system size or a critical coupling strength between cells. Such conditions could appear in a developing tissue due to the variations in the geometric constrains [75] or in the diffusion properties of the intercellular medium. In our results, we have shown that the final state of the cells can be influenced by a small external signal introducing a bias into the switch. A population of cells coupled by diffusible signaling responds in a hypersensitive way to the external signal, and may even display a response depending on the history of the signal concentration (hysteresis effect). One could envision that this 
phenomenon could be used in a developing tissue to achieve a robust and coordinated cell differentiation in response to small external signal, as for example a morphogen concentration. In a growing tissue, cells would be randomly switching between the stable states till reaching the critical size, and then transition to a fully coordinated population. In this case, the final fate of the cells could be determined by the concentration of the external signal. The process described here is purely hypothetical. Nevertheless, a phenomenon such as the community effect suggests that eukaryotic cells, during embryonic development, use cell-cell communication to coordinate the expression of a set of genes inside a group of cells. Whether cells in a developing tissue could differentiate collectively in a process similar to the Ising phase transition is an open question. 
Appendix 



\section{APPENDIX}

\section{A.I DYNAMIC CELL POPULATION STOCHASTIC SIMULATION SOFT- WARE: COLONY}

The Colony simulation software is a custom-developed software capable of simulating the stochastic dynamics of a population of cells communicating by QS diffusible signals and that includes cell growth and division. I have developed this software during my Ph.D. and have frequently updated it to add new functionalities. In the following I briefly describe its structure, its capabilities, and its use in producing the results presented in this thesis.

The code was written in $\mathrm{C}++$ in order to take advantage of the flexibility of classes to facilitate the writing and updating of the code. A summarized class diagram can be found in figure A.2. The basic idea behind the structure of the program is to separate the data structure defining the state of the cells from the algorithm used to compute the dynamics. The management of the changes in data structure due to cell division and cell deletion are made easier by the use of classes such as CellCollection. Another important point is that the cell data is kept together in a fixed-sized memory array in order to speed up the computation. This array is only modified when a cells divides or a cell is deleted. Several integrators are implemented and allow to compute the stochastic dynamics of cells using the Gillespie algorithm or a chemical Langevin algorithm. The definition of the system of reaction, parameters of the simulations and some analytical calculation are performed in Mathematica (version 9, Wolfram Research) which writes the input files and part of the source code for the stochastic algorithms. The software describes a homogeneous concentration of chemical species in the environment (milieu class), assuming well-mixed conditions in all the external volume. How- 


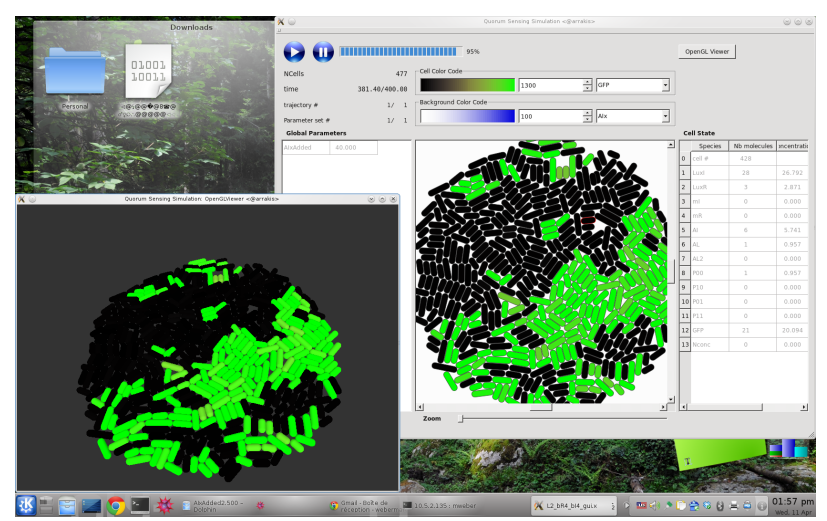

Figure A.1: Graphical user interface of the Colony software. The GUI allows the visualization in real-time of the species concentration in the population and in the environment, as well as parameters values and other relevant information.

ever, spatial diffusion of the autoinducer in the environment could be easily implemented. Although the modeling approach used in this thesis does not take into account spatial effects, the Colony software can compute cell movement using a rigid body dynamics engine. The cells movements have been computed in order to produce realistic movies of the growing population simulations, and are only used for graphical representation purposes with no scientific meaning. Nevertheless, this ability shows that the software would only need minimal modifications to implement spatial dynamics for cells (cell movement rules) and signaling molecules (diffusion). The software also integrates a graphical user interface which allows the visualization in real-time of the cell population and species concentrations (see figure A.I). More details on the software can be provided upon request to the author. 


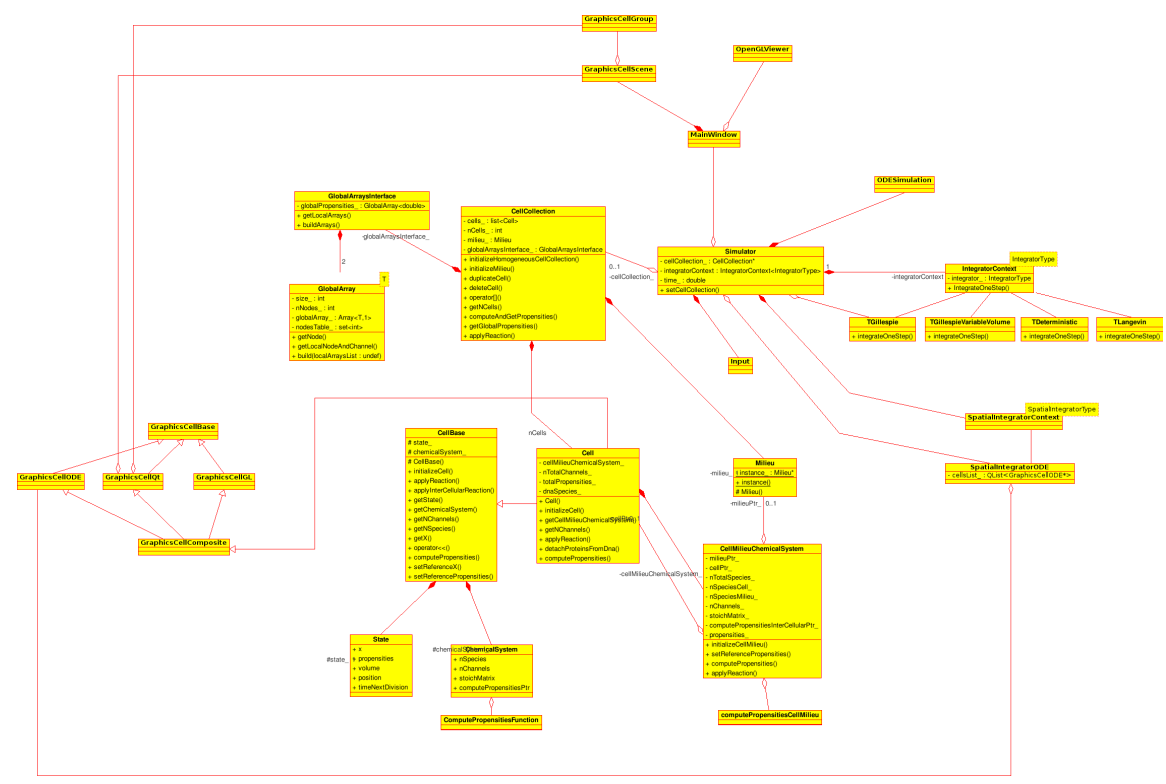

Figure A.2: Class diagram of the Colony software. The structure of the program allows to separate the data structure defining the state of the cells from the algorithm used to compute the dynamics. The management of the changes in data structure due to cell division and cell deletion are made easier by the use of classes such as CellCollection. Several stochastic integrators can be used, such as the Gillespie algorithm and the chemical Langevin algorithm. 
In chapter 5, the QS mechanism introduces a global coupling by allowing diffusible signals produced by one cell to reach all the other cells in the population. We seek to compare these results to the behaviour of the Ising model in the mean-field approach, where each spin is subjected to the same average interaction with the system. The Ising model presents a phase transition in the mean-field approach when varying the temperature $T$. However, the same phase transition can also be observed when varying the coupling coefficient J. In the following, we calculate the critical exponents of the mean-field Ising model when varying the coupling coefficient $J$.

Consider the general spin half Ising model given by the Hamiltonian,

$$
H=-\sum_{i} h_{i} S_{i}-\frac{1}{2} \sum_{i, j} J_{i j} S_{i} S_{j}
$$

where $S_{i}= \pm 1, h_{i}$ is the external magnetic field at site $i, J_{i j}$ the coupling coefficient between sites $i$ and $j$. We define the local magnetization

$$
m_{i}=\left\langle S_{i}\right\rangle
$$

The mean field approximation makes an expansion in the fluctuations,

$$
S_{i} S_{j}=\left[m_{i}+\left(S_{i}-m_{i}\right)\right]\left[m_{j}+\left(S_{j}-m_{j}\right)\right]
$$

so that

$$
\begin{aligned}
S_{i} S_{j}=m_{i} m_{j}+m_{i}\left(S_{j}-m_{j}\right)+m_{j} & \left(S_{i}-m_{i}\right) \\
& +\left(S_{i}-m_{i}\right)\left(S_{j}-m_{j}\right)
\end{aligned}
$$

The mean field approximation drops the term $\left(S_{i}-m_{i}\right)\left(S_{j}-m_{j}\right)$ which is quadratic in the fluctuations, so that,

$$
S_{i} S_{j} \approx m_{i} m_{j}+m_{i}\left(S_{j}-m_{j}\right)+m_{j}\left(S_{i}-m_{i}\right)
$$


The mean field Hamiltionian is then

$$
H_{m . f .}=-\sum_{i} h_{i} S_{i}-\frac{1}{2} \sum_{i, j} J_{i j}\left(m_{i} S_{j}+m_{j} S_{i}-m_{i} m_{j}\right)
$$

We consider now the case of the Ising ferromagnet with a translationally invariant lattice. In this case $m_{i}=m$ is the same everywhere and $\sum_{j} J_{i j}=J z$ is also assumed to be the same everywhere, with $z$ the coordination number of the lattice. The Hamiltonian reduces to

$$
\mathrm{H}_{\mathrm{m} . \mathrm{f} .}=-\frac{1}{2} \mathrm{~J} z \mathrm{Nm}^{2}-\sum_{i} S_{i}(\mathrm{Jzm}+\mathrm{h}) .
$$

The partition function writes

$$
Z_{\text {m.f. }}=e^{-\frac{1}{2} \beta J z N m^{2}}[2 \cosh (\beta J z m+\beta h)]^{N},
$$

the mean field Helmotz free energy,

$$
F_{\text {m.f. }}=N\left[\frac{1}{2} J z m^{2}-\frac{1}{\beta} \ln (2)-\frac{1}{\beta} \ln [\cosh (\beta J z m+\beta h)]\right] .
$$

We can write the mean field equation by deriving the value of the magnetization per spin,

$$
m=-\frac{1}{N}\left(\frac{\partial F_{m . f .}}{\partial h}\right)=\tanh (\beta J z m+\beta h)
$$

For $\beta \mathrm{J} z>1$, there are three solutions, while for $\beta \mathrm{J} z<1$, the only solution is $m=0$. The condition $\beta J z=1$ defines the critical temperature below which there is a finite magnetization at $h=0$. The critical temperature is $T_{c}=\frac{J z}{k}$. However, we are interested in calculating the critical exponents when varying the coupling coefficient $J$, not the temperature. Thus, we will consider the temperature $T$ as a constant parameter of the system and define $\tilde{J}=\mathrm{J} z$ as the control parameter. Hereafter we drop the tilde notation and use J. Then, the critical coupling coefficient is $\mathrm{J}_{\mathrm{c}}=\mathrm{kT}=\frac{1}{\beta}$.

We analyse the ferromagnetic solutions when $J$ is near the critical $J_{c}$, where $|\mathrm{m}| \sim 0$. Equation (A.10) writes, with $h=0$

$$
\mathrm{m}=\tanh \left(\frac{\mathrm{J}}{\mathrm{J}_{\mathrm{c}}} \mathrm{m}\right)
$$


which we develop for small $m, \tanh (x) \simeq x-\frac{x^{3}}{3}$,

$$
\mathrm{m}=\frac{\mathrm{J}}{\mathrm{J}_{\mathrm{c}}} \mathrm{m}-\frac{1}{3}\left(\frac{\mathrm{J}}{\mathrm{J}_{\mathrm{c}}} \mathrm{m}\right)^{3}
$$

Thus, either $m=0$ or

$$
\mathrm{m}^{2}=3\left(\frac{\mathrm{J}_{\mathrm{c}}}{\mathrm{J}}\right)^{3}\left(\frac{\mathrm{J}}{\mathrm{J}_{\mathrm{c}}}-1\right)
$$

We define the reduced coupling $j=\frac{J-J_{c}}{J_{c}}$,

$$
\mathrm{m}^{2}=3 \frac{j}{(1+j)^{3}}
$$

and when $j$ is small, we have

$$
\mathrm{m}^{2} \simeq 3 j
$$

Thus,

$$
\begin{array}{ll}
\mathrm{J}<\mathrm{J}_{\mathrm{c}} & \mathrm{m}=0 \\
\mathrm{~J}>\mathrm{J}_{\mathrm{c}} & \mathrm{m}= \pm(3|\mathrm{j}|)^{1 / 2}
\end{array}
$$

and the critical exponent is $\beta=1 / 2$, the same as in the temperature varying case.

We now compute the susceptibility,

$$
x=\left.\frac{\partial \mathrm{m}}{\partial \mathrm{h}}\right|_{\mathrm{h}=0}
$$

We expand (A.10) at third order in $m$ at first order in $h$,

$$
m \simeq \beta h+\beta J m-\beta^{3} J^{2} h m^{2}-\frac{1}{3} \beta^{3} J^{3} m^{3}
$$

Substitute $\beta$ by $1 / J_{c}$, then we derive w.r.t. $h$ at $h=0$,

$$
\chi=\left.\frac{\partial m}{\partial h}\right|_{h=0}=-\frac{J^{3} m^{2} \chi}{J_{c}^{3}}-\frac{J^{2} m^{2}}{J_{c}^{3}}+\frac{J x}{J_{c}}+\frac{1}{J_{c}}
$$


We find

$$
x=\frac{\mathrm{J}_{\mathrm{c}}^{2}-\mathrm{J}^{2} \mathrm{~m}^{2}}{-\mathrm{JJ}_{\mathrm{c}}^{2}+\mathrm{J}_{\mathrm{c}}^{3}+\mathrm{J}^{3} \mathrm{~m}^{2}}
$$

using the definition of the reduced coupling,

$$
x=\frac{1-(j+1)^{2} m^{2}}{(j+1)^{3} m^{2} J_{c}-j J_{c}}
$$

Then we find for small $j$ and using the appropriate expression for $m$ (either above or below the critical $\mathrm{J}_{\mathrm{c}}$ )

$$
\begin{aligned}
J<J_{c} \quad m & =0 \quad x=-\frac{1}{J_{c j}} \\
J>J_{c} \quad m & = \pm\left(3 \frac{j}{(1+j)^{3}}\right)^{1 / 2} \\
x^{+} & =\frac{1-2 j}{2 J_{c}\left(j+j^{2}\right)} \stackrel{j \rightarrow 0}{\simeq} \frac{1}{2 J_{c j}} \\
x^{-} & =\frac{1+4 j}{-4 J_{c}\left(j+j^{2}\right)} \stackrel{j \rightarrow 0}{\simeq} \frac{-1}{4 J_{c} j}
\end{aligned}
$$

In both cases the critical exponent is $\gamma=1$, the same as in the temperature varying case.

The behavior of the magnetization as a function of the external field at the critical temperature is the same as in the classical case because both the coupling coefficient and the temperature are fixed, we only look at the variation of the field $h$. Thus, $\delta=1 / 3$.

The critical exponent $\alpha$ is defined as $C_{h} \sim t^{-\alpha}$, or how the heat capacity at zero field behaves when approaching the critical temperature. For $\mathrm{J}<\mathrm{J}_{\mathrm{c}}\left(\mathrm{T}>\mathrm{T}_{\mathrm{c}}\right)$ we have $\mathrm{m}=0$ and the free energy (A.9) writes

$$
F_{m . f .}(N, h, T)=-N k T \ln 2
$$

Here we consider the temperature as a parameter of the system but we still consider derivatives w.r.t. $T$ in order to define the thermodynamic quantities. The heat capacity at constant field then reads

$$
C_{h}=-T \frac{\partial^{2} F_{m . f .}}{\partial T^{2}}=0
$$


and the critical exponent is $\alpha=0$. For $J>J_{c}\left(T<T_{c}\right)$ the dependence on $T$ is the same, so that $\alpha$ is still zero. Thus, the exponent $\alpha=0$ is the same as in the temperature varying case.

In summary, the critical exponents of the ferromagnetic Ising model in the mean-field approach when varying the coupling coefficient J are

$$
\begin{aligned}
& \gamma=1 \\
& \beta=1 / 2 \\
& \alpha=0 \\
& \delta=1 / 3
\end{aligned}
$$




\section{B.I INTRODUCCIÓ}

Des del punt de vista d'un físic, els sistemes biològics sorprenen per la seva gran complexitat a diferentes escales, des de el nivell molecular de proteïnes i ADN, fins a l'escala macroscòpica d'animals i plantes. A nivell molecular, les cèl-lules operen lluny de l'equilibri i es componen de xarxes complexes de gens i proteïnes que interactuen. La seva descripció des d'un punt de vista teòric és complexa. Tot i això, es necessiten mètodes i conceptes de la física per poder integrar tota la gran quantitat de dades que provenen de noves tècniques experimental i per poder entendre com funcionen els sistemes biològics. En aquesta tesi presentem el nostre model teòric i computacional per estudiar el funcionament d'alguns processos bàsics en els sistemes biològics: el soroll i la communicació entre cèl-lules.

Les fluctuacions estocàstiques, o soroll, son omnipresents en els sistemes biològics. Al llarg de la dècada passada, alguns estudis van mostrar que fluctuava el nivell $i$ activitat de les espècies en molts circuits reguladors [143]. Aquestes fluctuacions tenen el seu origen en el caràcter probabilístic de les reaccions químiques, que arribar a ser significatiu quan el número de molècules de les espècies químiques són molt baixos [82]. Es creu que els efectes estocàstics juguen també un paper important en la diferenciació cel-lular [12]. Així, el soroll permet a les cèlllules que estan exposades al mateix entorn triar entre destins diferents i, per tant, incrementa la diversitat fenotípica. En aquest sentit, el sistema regulador més simple i no trivial que mostra la multiestabilitat fenotípica correspon a un interruptor genètic amb dues possibles solucions estables: alta/baixa concentració de una proteïna reguladora. Aquest comportament s'ha 
trobat en sistemes biològics com per example la xarxa de regulació del metabolisme de la lactosa en E. coli [123], i també s'ha utilitzat en circuits sintètics $[56,76,8]$. En conseqüència, la caracterització dels interruptors genètics és important tant pel desenvolupament de circuits sintètics més grans i fiables [141] com per entendre processos complexes com la diferenciació cel·lular.

Durant molt de temps, s'ha pensat que els bacteris tenien una existència solitària. Ara, se sap que es comuniquen entre ells enviant i rebent missatges químics [13]. Aquest mecanisme de comunicació permet als bacteris sincronitzar la seva activitat a tota la colònia. A més a més, aquest comportament coordinat en alguns casos s'assembla al comportament d'organismes multicel.lulars, com per exemple l'anomenat "efecte de comunitat" en el desenvolupament [157]. En el mecanisme de quorum sensing (QS), les cèl-lules produixen, exporten i importen senyals moleculars (molècules autoinductores). Quan la colònia creix, hi ha més cèlllules que produixen i exporten molècules autoinductores, produiint un increment de la concentració de senyals moleculars en l'entorn i en les cèl-lules. Quan la concentració de molècules autoinductores arriba a un límit, aquestes activen la expressió dels gens controlats pel QS, coordinant les cèl-lules segons la densitat cel-lular en el medi. És important destacar, que el mecanisme de QS controla diversos canvis fenotípics important en els bacteris, com per exemple la virulència en $S$. aureus [7]. Actualment, aquest mecanisme es considera un sistema model per l'estudi de l'aparició del comportament coordinat entre cèl-lules que es comuniquen. A més a més, el QS ha obert tot un camp de recerca plena d'aplicacions tecnològiques molt prometadores [126], com per exemple la invasió de cèl-lules cancerígenes controlada de manera externa per l'entorn [3].

La coordinació per la comunicació de QS d'un gran nombre de bacteris, que expressen el mateix conjunt de gens d'una manera sincronitzada, comporta la qüestió de com aquesta coordinació es pot portar a terme malgrat les fluctuacions que afecten totes les xarxes biològiques de cada cèl-lula individual. Queden interessants preguntes sense respondre al voltant de la funció del soroll en sistemes de QS naturals i sintètics. Per exemple, la heterogeneïtat de la re- 
sposta cel-lular en moltes espècies de bacteris [136] al llarg de la transició de QS podria ser conseqüència de diferentes fonts de soroll, conegudes i desconegudes.

En aquesta tesi, intentem respondre aquestes preguntes utilitzant models matemàtics i simulacions estocàstiques. Ens centrem en interruptors genètics com a paradigma dels processos de decisió cel-lulars tant en sistemes de bacteris naturals com sintètics. Tenim com a objectiu l'estudi dels efectes del soroll en una cèl-lula individual i en l'aparició de comportament col-lectiu en poblacions de cèlllules que es comuniquen.

\section{B.2 RESUM DELS PRINCIPALS RESULTATS}

A continuació, farem un resum dels principals resultats de la tesi. El corresponent llistat de publicacions relacionat amb aquest treball es troba a la pàgina $\mathrm{v}$.

El nostre mètode es basa en la modelització matemàtica i en les simulacions estocàstiques, utilitzant tant com sigui possible dades experimental quantitatives. Hem usat mètodes determinístics ben establerts com les equacions cinètiques de reaccions químiques, mètodes estocàstics com l'algoritme de Gillespie [57], i mètodes de càlcul estocàstic analític per descriure les xarxes reguladores genètiques i el mecanisme de comunicació de QS. Per poder estudiar el comportament col-lectiu dels bacteris, hem desenvolupat un programa computacional capaç de simular la dinàmica estocàstica d'una població de cèl-lules que es comuniquen a través de senyals difusius i que te en compte el creixement i la divisió cel-lular (veieu appendix A.1). Aquesta eina computacional és original en el sentit que combina la dinàmica estocàstica de les xarxes reguladores genètiques, la comunicació entre cèl-lules i la dinàmica de proliferació cel-lular. Aquest programa ha estat usat al llarg de la tesi i ens ha permès analitzar els efectes del soroll bioquímic en la cèl-lula individual i en la població cel-lular.

Hem aplicat el nostre anàlisi a sistemes de diferents complexitats. Hem analitzat els següents sistemes, del més simple al més com- 
plexe: des d'un interruptor genètic autoactivador en una cèl-lula aîllada (capítol 4), una població de cèl-lules que expressen un senyal difusiu de QS de manera constitutiva (capítol 2), una població de cèl-lules amb l'interruptor genètic del sistema model LuxR/LuxI que es comuniquen a través del QS (capítol 3), fins a una població de cèl-lules amb un interruptor genètic de feedback negatiu que interactuen a través de l'intercanvi de dos senyals diferents de QS (capítol 5). En cadascun d'aquests sistemes, el nostre estudi ha contribuit a entendre millor els efectes de les fluctuacions en el comportament individual i col-lectiu de les cèl-lules. A continuació, resumim els resultats principals de cada capítol, en el ordre definit anteriorment. En la figura B.I, trobarem una visió de conjunt dels resultats de la tesi.

Al capítol 4, hem analitzat els efectes del soroll intrínsec en un interruptor genètic autoactivador en una cèl-lula aillada. Mentre els estudis previs mostraven que les fluctuacions poden modificar el paisatge epigènetic de l'interruptor genètic, faltava un formalisme teòric per entendre com les fluctuacions generen aquells canvis. Nosaltres hem desenvolupat una teoria perturbacional que mostra que el soroll afavoreix l'estabilitat del fenotip de l'estat "baix" de l'interruptor i que la regió de biestabilitat s'estén quan creix la intensitat de les fluctuacions (veieu figura B.IA), un efecte que hem anomenat estabilització estocàstica. Les nostres simulacions estocàstiques demostren que aquest efecte existeix tant en el model simple, utilitzat en l'anàlisi analític, com en un model més detallat que té en compte la reacció d'unió de la proteïna amb el promotor, suggerint que la estabilització estocàstica és un fenomen genèric en els interruptors de feedback positiu. Hem calculat la velocitat de l'activació de l'interruptor que ens ha permès entendre la relació entre el soroll intrínsec i la modificació estocàstica del paisatge epigenètic. Hem mostrat que incrementar el soroll intrínsec comporta incrementar la velocitat de les transicions de l'interruptor, però també modifica la barrera energètica entre els estats baix i alt, donant com a resultat un comportament complexe quant varia la intensitat del soroll. Els nostres resultats indiquen que la modificació del paisatge epigenètic deguda al soroll intrínsec, no només modifica els estats es- 
tables de l'interruptor, sinó també la dinàmica de les seves transicions. Aquest fenomen no acostuma a tenir-se en consideració en altres estudis teòrics. Els resultats presentats en el capítol 4 porten llum als efectes del soroll intrínsec en l'interruptor genètic autoactivador, de les següents maneres: i) aportant un anàlisi teòric que explica com i per què les fluctuacions bioquímiques modifiquen el paisatge epigenètic, i ii) explicant el comportament complexe de les transicions estocàstiques de l'interruptor quant varia la intensitat del soroll. Així, a nivell d'una cèl-lula individual, el soroll intrínsec contribueix a la variabilitat intercel-lular de l'interruptor genètic i pot modificar els seus estats estables i la seva dinàmica.

En el capítol 2, hem estudiat la interacció entre el soroll intracel-lular i el procés de difusió del mecanisme de comunicació del QS. Estudis previs mostren que aquesta difusió pot reduir el soroll a nivell del senyal molecular. Tot $i$ això, el paper que juguen les diferents fonts estocàstiques en la cèl-lula i les seves contribucions a la dinàmica de l'autoinductor no ha estat caracteritzada. Hem construit un model que descriu la expressió de la molècula autoinductora i la seva difusió en una població de cèl-lules. Ens hem centrat en la situació on el gen luxI s'expressa a un nivell constitutiu baix, de tal manera que podem assumir que: i) la regulació del feedback del gen luxI pot ser ignorat i ii) el nivell de transcripció és prou baix per assumir que com a màxim una molècula d'ARNm pot estar present en la cèl-lula. A més a més, assumim que la producció de l'autoinductor des de l'ARNm es pot descriure per una reacció d'un sol pas.

Basant-nos en aquestes suposicions, hem pogut derivar la expressió analítica de la distribució de l'autoinductor. Comparant els resultats analítics amb les simulacions estocàstiques, hem determinat les contribucions del soroll transcripcional (fluctuacions del número de còpies d'ARNm) i del soroll intrínsec en les fluctuacions de l'autoinductor. Hem mostrat que cambiar la velocitat de difusió produeix un repertori de dinàmiques per la molècula autoinductora (mireu figura B.1B). Els nostres resultats indiquen que la competició d'escales temporals entre la dinàmica de l'ARNm i el procés de difusió juga un paper crucial en la regulació de la quantitat total de soroll en el número de molècules autoinductores. Hem trobat que el soroll 
transcripcional és la principal contribució al soroll total per un rang ampli de valors de la velocitat de difusió. A més a més, hem observat que el soroll total presenta un màxim en funció de la velocitat de difusió, en contrast als estudis previs. Observant els valors experimentals de les velocitats de difusió en espècies bacterianes de QS, els nostres resultats apunten en la direcció que els bacteris han evolucionat per adaptar el seu mecanisme de comunicació i evitar les velocitats de difusió que maximitzen el soroll. En resum, al capítol 2, hem presentat un model reduit de la expressió genètica i de la comunicació del QS que ens ha permès: i) revelar la contribució del soroll intrínsec i del soroll transcripcional en les fluctuacions de la molècula autoactivadora, i ii) mostrar que la interacció entre la dinàmica de l'ARNm i el procés de difusió pot portar a una varietat de dinàmiques i de nivells de fluctuacions per la molècula autoinductora. Per tant, el mecanisme de comunicació del QS modifica les fluctuacions de la molècula autoinductora dins la cèlula i interactua amb el soroll de la expressió genètica.

En el capítol 3, hem estudiat els efectes del soroll de l'expressió genètica en la precisió de la coordinació de la població en la activació del sistema de QS LuxR/LuxI. La heterogeneïtat en la resposta de QS observada experimentalment en moltes espècies bacterianes porta a preguntar-se com la precisió de la coordinació de les cèl-lules depen de les fonts de soroll intracellular. Per respondre aquesta pregunta, ens hem centrat en una de les xarxes reguladores de QS més simples, el sistema LuxR/LuxI. A partir de models determinístics i estocàstics, hem analitzat la resposta i la dinàmica d'una població de cèl-lules a diferents nivells d'autoinductor. Els nostres resultats han mostrat que el soroll en la expressió genètica de LuxR és el principal factor que controla la variabilitat de l'activació del QS (mireu figura B.IC). Analitzant la dinàmica de la població, hem demostrat que la activació de l'interruptor del QS a prop de la concentració crítica d'autoinductor és molt lenta comparada amb la duració del cicle cel-lular, i, per tant, els efectes no-estacionaris són importants al llarg de la transició del QS. Hem mostrat que incrementar el soroll de la expressió genètica en LuxR disminueix la precisió transitòria de l'interruptor del QS. Aquests resultats, juntament amb algunes evidèn- 
cies experimentals sobre la regulació de LuxR en espècies de tipus silvestre, sugereixen que els bacteris han evolucionat cap a mecanismes per regular la intensitat del soroll en LuxR i per controlar la variabilitat en l'activació del QS. El nostre estudi aporta llum en la relació entre la dinàmica estocàstica d'una cèl-lula individual i el comportament col-lectiu en una població de cèl-lules que es comuniquen.

En el capítol 5, hem estudiat un nou mecanisme de coordinació en un model de toggle switch interconnectat amb dos vies de comunicació de QS. L'aplicació del mecanisme de comunicació del QS en biologia sintètica ha portat a la creació de circuits genètics que mostren una varietat de comportaments collectius. Hem presentat un model computacional d'una població de toggle switch que es comuniquen a través de l'intercanvi de dos senyals difusius de QS. La característica clau del nostre model resideix en l'acoblament tant dels estats "on" com "off" entre les cèl-lules. Hem mostrat que l'increment de la velocitat de difusió, que augmenta la força de l'acoblament entre les cèl-lules, porta a una transició de fase: va des d'una fase desordenada on les cèl-lules salten de manera aleatòria entre els dos estats de l'interruptor, fins a una fase ordenada amb totes les cèl-lules bloquejades en el mateix estat estable (mireu figura B.ID). La presència d'un pic en la susceptibilitat i el càlcul dels exponents crítics per finite size scaling confirmen l'existència de la transició de fase. La mateixa transició s'ha trobat en una població de cèlllules que creixen exponencialment en un volum tancat, amb totes les cèl-lules entrant en l'estat ordenat quan arriben a una mida crítica del sistema. A més a més, hem analitzat la resposta de les cèllules a un senyal extern que introdueix una asimetria en l'interruptor i hem mostrat que la resposta de les cèl-lules segueix una trajectòria d'histèresi, similar a la resposta del model ferromagnètic d'Ising a un camp magnètic extern. Hem trobat que per una velocitat de difusió per sota del punt crític, l'acoblament entre cèl-lules intensifica la sensibilitat de la resposta de la població i hem suggerit que aquest nou mecanisme podria ser usat per augmentar la fiabilitat i sensibilitat de biosensors. Els nostres resultats suggereixen un nou mecanisme per la decisió cel-lular col-lectiva basat en el fenomen de la transició de fase. 
A
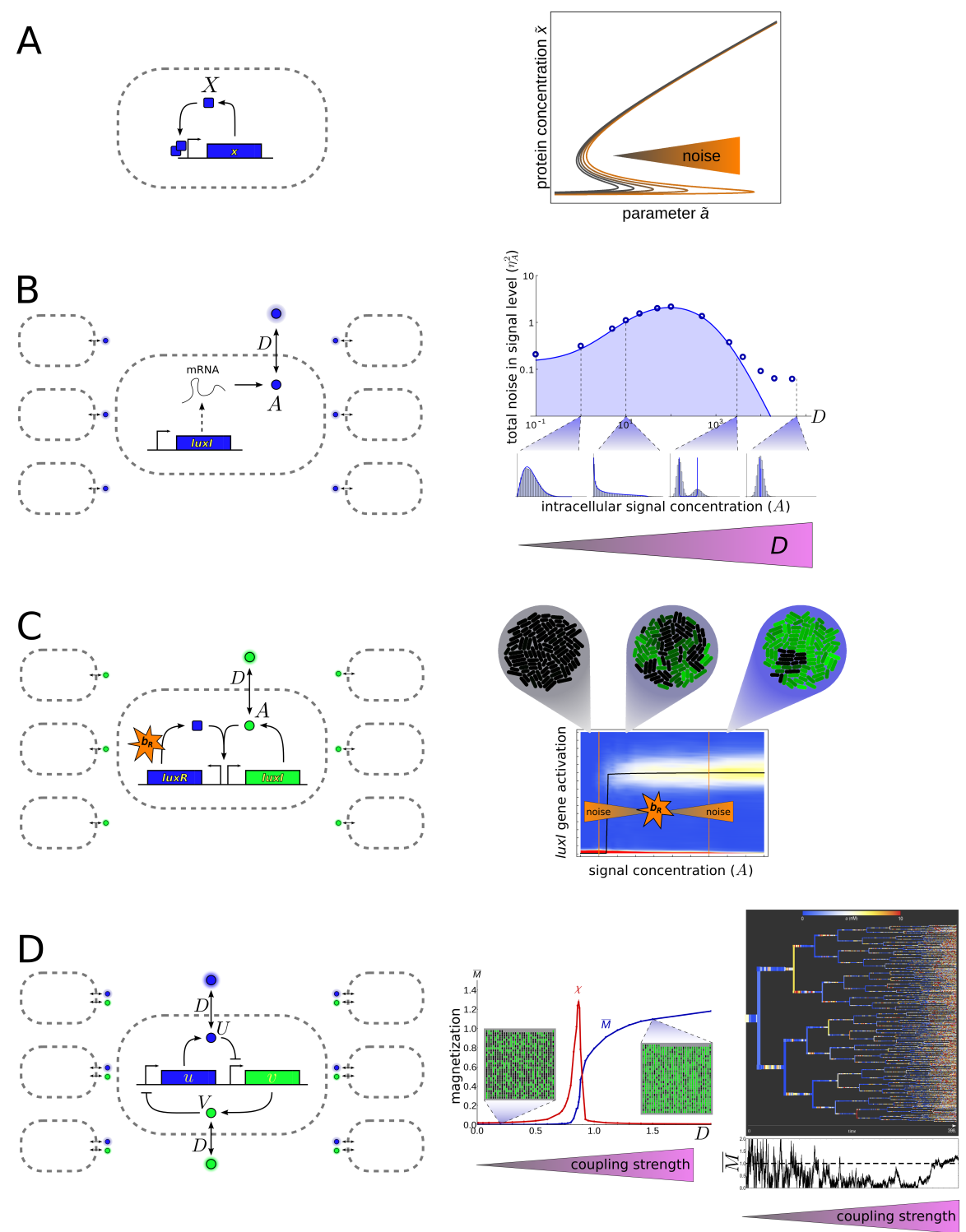

Figura B.1: Il-lustració dels principals resultats de la tesi. Els principals resultats de cada capítol es presenten segons la complexitat del model. Veieu el text principal pels detalls. (A) Estabilització estocàstica dels estats fenotípics: l'interruptor genètic biestable com a exemple (capítol 4). (B) Regulació del soroll pel quorum sensing en sistemes amb baix número de còpies de ARNm (capítol 4). (C) Dinàmica de l'interruptor de QS: efectes estocàstics i noestacionaris (capítol 3). (D) Transició de fase en el toggle switch acoblat per QS: un model de Ising en bactèries (capítol 5). 


\section{BIBLIOGR A PHY}

[1] Murat Acar, Attila Becskei, and Alexander van Oudenaarden. Enhancement of cellular memory by reducing stochastic transitions. Nature, 435(7039):22832, May 2005. doi: 10.1038/natureo3524. Available online: link. (Cited on pages 13 and 104.)

[2] Murat Acar, Jerome T Mettetal, and Alexander van Oudenaarden. Stochastic switching as a survival strategy in fluctuating environments. Nature genetics, 40(4):471-5, April 2008. doi: 10.1038/ng.110. Available online: link. (Cited on page 17.$)$

[3] J Christopher Anderson, Elizabeth J Clarke, Adam P Arkin, and Christopher a Voigt. Environmentally controlled invasion of cancer cells by engineered bacteria. Journal of molecular biology, 355(4):619-27, January 2006. doi: 10.1016/j.jmb.2005.10.076. Available online: link. (Cited on pages 27 and 204.)

[4] Kelsi L Anderson and Paul M Dunman. Messenger RNA Turnover Processes in Escherichia coli, Bacillus subtilis, and Emerging Studies in Staphylococcus aureus. International journal of microbiology, 2009:525491, 2009. doi: 10.1155/ 2009/525491. (Cited on page 49.)

[5] Claudia Anetzberger, Torsten Pirch, and Kirsten Jung. Heterogeneity in quorum sensing-regulated bioluminescence of Vibrio harveyi. Molecular microbiology, 73(2):267-77, 2009. doi: 10.1111/j.1365-2958.2009.06768.x. Available online: link. (Cited on pages 34 and 67.)

[6] Claudia Anetzberger, Ursula Schell, and Kirsten Jung. Single cell analysis of Vibrio harveyi uncovers functional heterogeneity in response to quorum sensing signals. BMC microbiology, 12(1):209, September 2012. doi: 10.1186/ 1471-2180-12-209. Available online: link. (Cited on page 104.)

[7] L Caetano M Antunes, Rosana B R Ferreira, Michelle M C Buckner, and B Brett Finlay. Quorum sensing in bacterial virulence. Microbiology (Reading, England), 156(Pt 8):2271-82, August 2010. doi: 10.1099/mic.0.038794-o. Available online: link. (Cited on page 204.)

[8] M.R. Atkinson, M.A. Savageau, J.T. Myers, and A.J. Ninfa. Development of genetic circuitry exhibiting toggle switch or oscillatory behavior in Escherichia coli. Cell, 113(5):597-607, 2003. Available online: link. (Cited on pages $13,109,143$, and 204.) 
[9] D W Austin, M S Allen, J M McCollum, R D Dar, J R Wilgus, G S Sayler, N F Samatova, C D Cox, and M L Simpson. Gene network shaping of inherent noise spectra. Nature, 439(7076):608-11, March 2006. doi: 10.1038/nature04194. Available online: link. (Cited on page 13.)

[10] Maier S Avendaño, Chad Leidy, and Juan M Pedraza. Tuning the range and stability of multiple phenotypic states with coupled positive-negative feedback loops. Nature communications, 4:2605, November 2013. doi: 10.1038/ ncomms3605. Available online: link. (Cited on pages 13, 14, and 136.)

[11] Frederick K Balagaddé, Hao Song, Jun Ozaki, Cynthia H Collins, Matthew Barnet, Frances H Arnold, Stephen R Quake, and Lingchong You. A synthetic Escherichia coli predator-prey ecosystem. Molecular systems biology, 4(187): 187, 2008. doi: 10.1038/msb.2008.24. (Cited on page 176.)

[12] Gábor Balázsi, Alexander van Oudenaarden, and James J Collins. Cellular decision making and biological noise: from microbes to mammals. Cell, 144 (6):910-25, March 2011. doi: 10.1016/j.cell.2011.01.030. Available online: link. (Cited on pages 103, 105, 109, and 203.)

[13] Bonnie L Bassler and Richard Losick. Bacterially speaking. Cell, 125(2):23746, 2006. doi: 10.1016/j.cell.2006.04.001. (Cited on pages 20, 24, and 204.)

[14] Bonnie L Bassler and Melissa B Miller. The Prokaryotes, volume 2. Springer Berlin Heidelberg, Berlin, Heidelberg, 2013. ISBN 978-3-642-30122-3. doi: 10.1007/978-3-642-30123-0. Available online: link. (Cited on pages 21, 22, 24, and 26.)

[15] S. Basu, Y. Gerchman, C.H. Collins, F.H. Arnold, and R. Weiss. A synthetic multicellular system for programmed pattern formation. Nature, 434(7037): 1130-1134, 2005. (Cited on pages 27 and 28.)

[16] Nicole a Becker, Justin P Peters, L James Maher, and Troy a Lionberger. Mechanism of promoter repression by Lac repressor-DNA loops. Nucleic acids research, 41(1):156-66, January 2013. doi: 10.1093/nar/gks1011. Available online: link. (Cited on page 4.)

[17] a Becskei and L Serrano. Engineering stability in gene networks by autoregulation. Nature, 405(6786):590-3, June 2000. doi: 10.1038/35014651. Available online: link. (Cited on page 13.)

[18] a Becskei, B Séraphin, and L Serrano. Positive feedback in eukaryotic gene networks: cell differentiation by graded to binary response conversion. The EMBO journal, 20(10):2528-35, May 2001. doi: 10.1093/emboj/20.10.2528. Available online: link. (Cited on page 13.) 
[19] Matthew R Bennett and Jeff Hasty. Microfluidic devices for measuring gene network dynamics in single cells. Nature reviews. Genetics, 10(9):628-38, 2009. doi: $10.1038 / \mathrm{nrg} 2625$. (Cited on pages 48 and 79 .)

[20] O G Berg. A model for the statistical fluctuations of protein numbers in a microbial population. Journal of theoretical biology, 71(4):587-603, April 1978. Available online: link. (Cited on pages 6 and 7.)

[21] K Binder. Critical properties from Monte Carlo coarse graining and renormalization. Physical Review Letters, 47(August):693-696, 1981. Available online: link. (Cited on page 155.)

[22] Marc R Birtwistle, Jens Rauch, Anatoly Kiyatkin, Edita Aksamitiene, Maciej Dobrzynski, Jan B Hoek, Walter Kolch, Babatunde a Ogunnaike, and Boris N Kholodenko. Emergence of bimodal cell population responses from the interplay between analog single-cell signaling and protein expression noise. BMC systems biology, 6:109, January 2012. doi: 10.1186/1752-0509-6-109. Available online: link. (Cited on pages 110 and 137.)

[23] William J Blake, Gábor Balázsi, Michael a Kohanski, Farren J Isaacs, Kevin F Murphy, Yina Kuang, Charles R Cantor, David R Walt, and James J Collins. Phenotypic consequences of promoter-mediated transcriptional noise. Molecular cell, 24(6):853-65, December 2006. doi: 10.1016/j.molcel.2006.11.003. Available online: link. (Cited on page 17.)

[24] James Q Boedicker, Meghan E Vincent, and Rustem F Ismagilov. Microfluidic confinement of single cells of bacteria in small volumes initiates highdensity behavior of quorum sensing and growth and reveals its variability. Angewandte Chemie (International ed. in English), 48(32):5908-11, 2009. doi: 10.1002/anie.200901550. (Cited on pages 34, 48,67, and 191.)

[25] Hamid Bolouri and Eric H Davidson. The gene regulatory network basis of the "community effect," and analysis of a sea urchin embryo example. Developmental biology, 340(2):170-8, April 2010. doi: 10.1016/j.ydbio.2009.06. 007. Available online: link. (Cited on page 139.)

[26] J J Bourgois, F E Sluse, F A Baguet, and J Mallefet. Kinetics of Light Emission and Oxygen Consumption by Bioluminescent Bacteria. Journal of bioenergetics and biomembranes, 33(4), 2001. doi: 0145-479X/01/080o-0353. (Cited on page 22.)

[27] Clifford P Brangwynne, Christian R Eckmann, David S Courson, Agata Rybarska, Carsten Hoege, Jöbin Gharakhani, Frank Jülicher, and Anthony a Hyman. Germline P granules are liquid droplets that localize by controlled dissolution/condensation. Science (New York, N.Y.), 324(5935):1729-32, June 2009. doi: 10.1126/science.1172046. Available online: link. (Cited on page 141.) 
[28] Katie Brenner, David K Karig, Ron Weiss, and Frances H Arnold. Engineered bidirectional communication mediates a consensus in a microbial biofilm consortium. Proceedings of the National Academy of Sciences of the United States of America, 104(44):17300-4, October 2007. doi: 10.1073/pnas.0704256104. Available online: link. (Cited on page 176.)

[29] FJ Bruggeman and N Blüthgen. Noise management by molecular networks. PLoS Computational, 5(9):26-28, 2009. doi: 10.1371/Citation. Available online: link. (Cited on page 15.)

[30] Long Cai, Nir Friedman, and X Sunney Xie. Stochastic protein expression in individual cells at the single molecule level. Nature, 440(7082):358-62, 2006. doi: 10.1038/nature04599. Available online: link. (Cited on pages 6, 7, 52, 79, 83 , and 136.)

[31] Long Cai, Chiraj K Dalal, and Michael B Elowitz. Frequency-modulated nuclear localization bursts coordinate gene regulation. Nature, 455(7212):48590, September 2008. doi: 10.1038/nature07292. Available online: link. (Cited on page 16.)

[32] Oriol Canela-Xandri, Francesc Sagués, and Javier Buceta. Interplay between intrinsic noise and the stochasticity of the cell cycle in bacterial colonies. Biophysical journal, 98(11):2459-68, June 2010. doi: 10.1016/j.bpj.2010.02.045. Available online: link. (Cited on pages 63 and 78.)

[33] Eric C Carnes, Deanna M Lopez, Niles P Donegan, Ambrose Cheung, Hattie Gresham, Graham S Timmins, and C Jeffrey Brinker. Confinement-induced quorum sensing of individual Staphylococcus aureus bacteria. Nature chemical biology, 6(1):41-5, 2010. doi: 10.1038/nchembio.264. (Cited on pages 48 and 191.)

[34] Jeffrey R Chabot, Juan M Pedraza, Prashant Luitel, and Alexander van Oudenaarden. Stochastic gene expression out-of-steady-state in the cyanobacterial circadian clock. Nature, 450(7173):1249-52, December 2007. doi: 10.1038/natureo6395. Available online: link. (Cited on page 10.)

[35] Guilhem Chalancon, Charles N J Ravarani, S Balaji, Alfonso Martinez-Arias, L Aravind, Raja Jothi, and M Madan Babu. Interplay between gene expression noise and regulatory network architecture. Trends in genetics : TIG, 28(5): 221-232, February 2012. doi: 10.1016/j.tig.2012.01.006. Available online: link. (Cited on page 15.)

[36] Paul J Choi, Long Cai, Kirsten Frieda, and X Sunney Xie. A stochastic singlemolecule event triggers phenotype switching of a bacterial cell. Science (New York, N.Y.), 322(5900):442-6, 2009. doi: 10.1126/science.1161427. Available online: link. (Cited on pages 4,38 , and 136.) 
[37] Swati Choudhary and Claudia Schmidt-Dannert. Applications of quorum sensing in biotechnology. Applied microbiology and biotechnology, 86(5):126779, May 2010. doi: 10.1007/s00253-010-2521-7. Available online: link. (Cited on page 27.)

[38] Jodi L Connell, Aimee K Wessel, Matthew R Parsek, Andrew D Ellington, Marvin Whiteley, and Jason B Shear. Probing prokaryotic social behaviors with bacterial "lobster traps". mBio, 1(4), January 2010. doi: 10.1128/mBio. 00202-10. Available online: link. (Cited on page 151.)

[39] C.D. Cox, G.D. Peterson, M.S. Allen, J.M. Lancaster, J.M. McCollum, D. Austin, L. Yan, G.S. Sayler, and M.L. Simpson. Analysis of noise in quorum sensing. OMICS : a journal of integrative biology, 7(3):317-334, 2003. Available online: link. (Cited on pages 26 and 69.)

[40] Wendy J Crookes, Lin-Lin Ding, Qing Ling Huang, Jennifer R Kimbell, Joseph Horwitz, and Margaret J McFall-Ngai. Reflectins: the unusual proteins of squid reflective tissues. Science (New York, N.Y.), 303(5655):235-8, January 2004. doi: 10.1126/science.1091288. Available online: link. (Cited on page 21.)

[41] Tal Danino, Octavio Mondragón-Palomino, Lev Tsimring, and Jeff Hasty. A synchronized quorum of genetic clocks. Nature, 463(7279):326-330, 2010. doi: 10.1038/natureo8753. (Cited on pages 27, 31, 68, 139, 181, and 190.)

[42] T. R. de Kievit and Barbara H Iglewski. Bacterial Quorum Sensing in Pathogenic Relationships. Infection and Immunity, 68(9):4839-4849, September 2000. doi: 10.1128/IAI.68.9.4839-4849.2000. Available online: link. (Cited on page 22.)

[43] Giorgio Dieci and André Sentenac. Detours and shortcuts to transcription reinitiation. Trends in biochemical sciences, 28(4):202-9, April 2003. doi: 10. 1016/Sog68-ooo4(03)ooo54-9. Available online: link. (Cited on page 6.)

[44] Gabriel E. Dilanji, Jessica B. Langebrake, Patrick De Leenheer, and Stephen J. Hagen. Quorum Activation at a Distance: Spatiotemporal Patterns of Gene Regulation from Diffusion of an Autoinducer Signal. Journal of the American Chemical Society, 134(12):5618-5626, March 2012. doi: 10.1021/ja211593q. Available online: link. (Cited on pages 181 and 190.)

[45] J D Dockery and J P Keener. A mathematical model for quorum sensing in Pseudomonas aeruginosa. Bulletin of mathematical biology, 63(1):95-116, 2001. doi: 10.1006/bulm.2000.0205. Available online: link. (Cited on pages 26 and 69.) 
[46] Yann Dublanche, Konstantinos Michalodimitrakis, Nico Kümmerer, Mathilde Foglierini, and Luis Serrano. Noise in transcription negative feedback loops: simulation and experimental analysis. Molecular systems biology, 2:41, January 2006. doi: 10.1038/msb4100081. Available online: link. (Cited on page 13.)

[47] A. Eberhard, A. L. Burlingame, C. Eberhard, G. L. Kenyon, K. H. Nealson, and N. J. Oppenheimer. Structural Identification of Autoinducer of Photobacterium fischeri Luciferase. Biochemistry, 20:2444-2449, 1981. (Cited on page 21.)

[48] Avigdor Eldar and Michael B. Elowitz. Functional roles for noise in genetic circuits. Nature, 467(7312):167-173, September 2010. doi: 10.1038/ natureo9326. Available online: link. (Cited on pages 15 and 16.)

[49] Johan Elf, Gene-Wei Li, and X Sunney Xie. Probing transcription factor dynamics at the single-molecule level in a living cell. Science (New York, N.Y.), 316(5828):1191-4, 2007. doi: 10.1126/science.1141967. Available online: link. (Cited on page 4.)

[50] Michael B Elowitz, Arnold J Levine, Eric D Siggia, and Peter S Swain. Stochastic gene expression in a single cell. Science (New York, N.Y.), 297(5584):1183-6, 2002. doi: 10.1126/science.1070919. (Cited on pages $11,12,60$, and 63.)

[51] Nir Friedman, Long Cai, and X. Xie. Linking Stochastic Dynamics to Population Distribution: An Analytical Framework of Gene Expression. Physical Review Letters, 97(16):168302, 2006. doi: 10.1103/PhysRevLett.97.168302. Available online: link. (Cited on pages 7 and 52.)

[52] David Frigola, Laura Casanellas, José M Sancho, and Marta Ibañes. Asymmetric stochastic switching driven by intrinsic molecular noise. PLoS One, 7 (2):e31407, January 2012. doi: 10.1371/journal.pone.0031407. Available online: link. (Cited on pages 14, 35, 68, 104, 110, 118, and 120.)

[53] J. Garcia-Ojalvo, M.B. Elowitz, and S.H. Strogatz. Modeling a synthetic multicellular clock: Repressilators coupled by quorum sensing. Proceedings of the National Academy of Sciences of the United States of America, 101(30):10955, 2004. (Cited on pages 31 and 68.)

[54] Jordi Garcia-Ojalvo and José M Sancho. Noise in Spatially Extended Systems. Springer-Verlag, 1999. (Cited on page 157.)

[55] C.W. Gardiner. Handbook of Stochastic Methods: for Physics, Chemistry and the Natural Sciences. Springer, 2nd editio edition, 1985. ISBN 3540208828. (Cited on page 130.) 
[56] T S Gardner, C R Cantor, and J J Collins. Construction of a genetic toggle switch in Escherichia coli. Nature, 403(6767):339-42, January 2000. doi: 10. 1038/35002131. Available online: link. (Cited on pages 13, 109, 141, 143, 144, and 204.)

[57] D.T. Gillespie. Exact stochastic simulation of coupled chemical reactions. The journal of physical chemistry, 81(25):2340-2361, 1977. (Cited on pages 8, 33, 41, $74,76,88,127,145,183$, and 205.)

[58] D.T. Gillespie. The chemical Langevin equation. The Journal of Chemical Physics, 113(1):297, 2000. Available online: link. (Cited on pages 112 and 120.)

[59] Aaron D Goldberg, C David Allis, and Emily Bernstein. Epigenetics: a landscape takes shape. Cell, 128(4):635-8, February 2007. doi: 10.1016/j.cell.2007. 02.006. Available online: link. (Cited on page 109.)

[6o] Ido Golding, Johan Paulsson, Scott M Zawilski, and Edward C Cox. Realtime kinetics of gene activity in individual bacteria. Cell, 123(6):1025-36, 2005. doi: 10.1016/j.cell.2005.09.031. (Cited on pages 5 and 77.)

[61] Didier Gonze and Albert Goldbeter. Circadian rhythms and molecular noise. Chaos (Woodbury, N.Y.), 16(2):026110, June 2006. doi: 10.1063/1.2211767. Available online: link. (Cited on page 32.)

[62] A B Goryachev, D J Toh, and T Lee. Systems analysis of a quorum sensing network: design constraints imposed by the functional requirements, network topology and kinetic constants. Bio Systems, 83(2-3):178-87, 2006. doi: 10.1016/j.biosystems.2005.04.006. Available online: link. (Cited on pages 26 and 69.)

[63] Andrew B Goryachev. Understanding bacterial cell-cell communication with computational modeling. Chemical reviews, 111(1):238-50, January 2011. doi: 10.1021/cr100286z. Available online: link. (Cited on pages 28 and 31.)

[64] Andrew B Goryachev, Da-Jun Toh, Keng Boon Wee, Hai-Bao Zhang, LianHui Zhang, and Travis Lee. Transition to quorum sensing in an Agrobacterium population: A stochastic model. PLoS computational biology, 1(4):e37, 2005. doi: 10.1371/journal.pcbi.oo10037. (Cited on pages 69, 77, and 104.)

[65] Stephen J. Hagen, Minjun Son, Joel T. Weiss, and Jonathan H. Young. Bacterium in a box: sensing of quorum and environment by the LuxI/LuxR gene regulatory circuit. Journal of Biological Physics, 36(3):317-327, February 2010. doi: 10.1007/s10867-010-9186-4. (Cited on pages 34, 48, 67, and 191.)

[66] Eric L Haseltine and Frances $\mathrm{H}$ Arnold. Implications of rewiring bacterial quorum sensing. Applied and environmental microbiology, 74(2):437-45, 2008. 
doi: 10.1128/AEM.o1688-07. Available online: link. (Cited on pages 26 and 69.)

[67] JW Hastings, WH Riley, and J Massa. Purification, properties, and chemiluminescence quantum yield of bacterial luciferase. Journal of Biological Chemistry, 240:1473-1481, 1965. (Cited on page 22.)

[68] J Hasty, J Pradines, M Dolnik, and J J Collins. Noise-based switches and amplifiers for gene expression. Proceedings of the National Academy of Sciences of the United States of America, 97(5):2075-80, March 2000. doi: 10.1073/pnas. 040411297. Available online: link. (Cited on page 14.)

[69] M. Herzberg, I.K. Kaye, W. Peti, and T.K. Wood. YdgG (TqsA) controls biofilm formation in Escherichia coli K-12 through autoinducer 2 transport. Journal of bacteriology, 188(2):587, 2006. doi: 10.1128/JB.188.2.587. (Cited on page 51.)

[70] Douglas a Higgins, Megan E Pomianek, Christina M Kraml, Ronald K Taylor, Martin F Semmelhack, and Bonnie L Bassler. The major Vibrio cholerae autoinducer and its role in virulence factor production. Nature, 450(7171):883-6, December 2007. doi: 10.1038/nature06284. Available online: link. (Cited on page 22.)

[71] Dawei Hong, William M Saidel, Shushuang Man, and Joseph V Martin. Extracellular noise-induced stochastic synchronization in heterogeneous quorum sensing network. Journal of theoretical biology, 245(4):726-36, 2007. doi: 10.1016/j.jtbi.2006.12.006. Available online: link. (Cited on pages 64, 101, and 190.)

[72] Seok Hoon Hong, Manjunath Hegde, Jeongyun Kim, Xiaoxue Wang, Arul Jayaraman, and Thomas K Wood. Synthetic quorum-sensing circuit to control consortial biofilm formation and dispersal in a microfluidic device. Nature communications, 3:613, January 2012. doi: 10.1038/ncomms1616. Available online: link. (Cited on page 176.)

[73] Werner Horsthemke and R. Lefever. Noise-induced transitions: theory and applications in physics, chemistry and biology. Springer-Verlag, Berlin Heidelberg, 1984. (Cited on pages 6, 38, 39, 40, 44, 112, 116, and 117.)

[74] Sui Huang, Gabriel Eichler, Yaneer Bar-Yam, and Donald E. Ingber. Cell Fates as High-Dimensional Attractor States of a Complex Gene Regulatory Network. Physical Review Letters, 94(12):128701, April 2005. doi: 10.1103/ PhysRevLett.94.128701. Available online: link. (Cited on page 109.)

[75] K D Irvine and C Rauskolb. Boundaries in development: formation and function. Annual review of cell and developmental biology, 17:189-214, January 
2001. doi: 10.1146/annurev.cellbio.17.1.189. Available online: link. (Cited on page 191.)

[76] Farren J Isaacs, Jeff Hasty, Charles R Cantor, and J J Collins. Prediction and measurement of an autoregulatory genetic module. Proceedings of the National Academy of Sciences of the United States of America, 100(13):7714-9, June 2003. doi: 10.1073/pnas.1332628100. Available online: link. (Cited on pages 13, 109, 118, and 204.)

[77] Srividya Iyer-Biswas, F. Hayot, and C. Jayaprakash. Stochasticity of gene products from transcriptional pulsing. Physical Review E, 79(3):1-9, 2009. doi: 10.1103/PhysRevE.79.031911. (Cited on pages 43 and 61.)

[78] Seunghee S Jang, Kevin T Oishi, Robert G Egbert, and Eric Klavins. Specification and simulation of synthetic multicelled behaviors. ACS synthetic biology, I(8):365-74, August 2012. doi: 10.1021/sb300034m. Available online: link. (Cited on page 69.)

[79] Joanna Jaruszewicz, Pawel J Zuk, and Tomasz Lipniacki. Type of noise defines global attractors in bistable molecular regulatory systems. Journal of theoretical biology, 317:140-51, January 2013. doi: 10.1016/j.jtbi.2012.10.004. Available online: link. (Cited on pages 35, 110, 127, and 135.)

[8o] B. W. Jones and M. K. Nishiguchi. Counterillumination in the Hawaiian bobtail squid, Euprymna scolopes Berry (Mollusca: Cephalopoda). Marine Biology, 144(6):1151-1155, June 2004. doi: 10.1007/soo227-003-1285-3. Available online: link. (Cited on page 21.)

[81] Brooke a Jude, Raquel M Martinez, Karen Skorupski, and Ronald K Taylor. Levels of the secreted Vibrio cholerae attachment factor GbpA are modulated by quorum-sensing-induced proteolysis. Journal of bacteriology, 191(22):69117, November 2009. doi: 10.1128/JB.00747-09. Available online: link. (Cited on page 22.)

[82] Mads Kaern, Timothy C Elston, William J Blake, and James J Collins. Stochasticity in gene expression: from theories to phenotypes. Nature reviews. Genetics, 6(6):451-64, 2005. doi: 10.1038/nrg1615. (Cited on pages 3, 5, 8, 10, 37, 68, 79 , and 203.)

[83] Tibor Kalmar, Chea Lim, Penelope Hayward, Silvia Muñoz Descalzo, Jennifer Nichols, Jordi Garcia-Ojalvo, and Alfonso Martinez Arias. Regulated fluctuations in nanog expression mediate cell fate decisions in embryonic stem cells. PLoS biology, 7(7):e1000149, July 2009. doi: 10.1371/journal.pbio.1000149. Available online: link. (Cited on page 137.) 
[84] HB Kaplan and EP Greenberg. Diffusion of autoinducer is involved in regulation of the Vibrio fischeri luminescence system. Journal of bacteriology, 163 (3):1210, 1985. Available online: link. (Cited on pages 25, 26, 31, and 83.)

[85] Diana Karlsson, Stefan Karlsson, Erik Gustafsson, Birgitta Henriques Normark, and Patric Nilsson. Modeling the regulation of the competenceevoking quorum sensing network in Streptococcus pneumoniae. BioSystems, 90(1):211-23, 2007. doi: 10.1016/j.biosystems.2006.08.005. Available online: link. (Cited on page 69.)

[86] Rajesh Karmakar and Indrani Bose. Graded and binary responses in stochastic gene expression. Physical biology, 1(3-4):197-204, 2004. doi: 10.1088/ 1478-3967/1/4/oo1. (Cited on page 43.)

[87] G.F. Kaufmann, R. Sartorio, S.H. Lee, C.J. Rogers, M.M. Meijler, J.A. Moss, B. Clapham, A.P. Brogan, T.J. Dickerson, and K.D. Janda. Revisiting quorum sensing: discovery of additional chemical and biological functions for 3-oxo$\mathrm{N}$-acylhomoserine lactones. Proceedings of the National Academy of Sciences of the United States of America, 102(2):309, 2005. (Cited on pages 48 and 83.)

[88] T.B. Kepler and T.C. Elston. Stochasticity in transcriptional regulation: origins, consequences, and mathematical representations. Biophysical Journal, 81 (6):3116-3136, 2001. (Cited on pages 5, 35, 110, and 130.)

[89] Caroline H Ko, Yujiro R Yamada, David K Welsh, Ethan D Buhr, Andrew C Liu, Eric E Zhang, Martin R Ralph, Steve a Kay, Daniel B Forger, and Joseph S Takahashi. Emergence of noise-induced oscillations in the central circadian pacemaker. PLoS biology, 8(10):e1000513, January 2010. doi: 10.1371/journal. pbio.1000513. Available online: link. (Cited on page 32.)

[9o] H. Kobayashi, M. Kæ rn, M. Araki, K. Chung, T.S. Gardner, C.R. Cantor, and J.J. Collins. Programmable cells: interfacing natural and engineered gene networks. Proceedings of the National Academy of Sciences, 101(22):8414, 2004. Available online: link. (Cited on pages 35, 141, 143, 145, 174, 176, and 177.)

[91] Aneta Koseska, Alexey Zaikin, Jürgen Kurths, and Jordi García-Ojalvo. Timing cellular decision making under noise via cell-cell communication. PLoS One, 4(3): 4872 , 2009. doi: 10.1371/journal.pone.0004872. (Cited on pages 31, $65,69,141,177$, and 180.)

[92] Edo Kussell and Stanislas Leibler. Phenotypic diversity, population growth, and information in fluctuating environments. Science (New York, N.Y.), 309 (5743):2075-8, September 2005. doi: 10.1126/science.1114383. Available online: link. (Cited on page 17.) 
[93] Christina Kuttler and Burkhard A Hense. Interplay of two quorum sensing regulation systems of Vibrio fischeri. Journal of theoretical biology, 251(1):16780, 2008. doi: 10.1016/j.jtbi.2007.11.015. Available online: link. (Cited on page 69.)

[94] Breah LaSarre and Michael J Federle. Exploiting quorum sensing to confuse bacterial pathogens. Microbiology and molecular biology reviews : MMBR, 77(1): 73-111, March 2013. doi: 10.1128/MMBR.ooo46-12. Available online: link. (Cited on pages 26 and 30.)

[95] Andrée M Lazdunski, Isabelle Ventre, and James N Sturgis. Regulatory circuits and communication in Gram-negative bacteria. Nature reviews. Microbiology, 2(7):581-92, 2004. doi: 10.1038/nrmicro924. Available online: link. (Cited on page 25.)

[96] Thuc T Le, Sébastien Harlepp, Calin C Guet, Kimberly Dittmar, Thierry Emonet, Tao Pan, and Philippe Cluzel. Real-time RNA profiling within a single bacterium. Proceedings of the National Academy of Sciences of the United States of America, 102(26):9160-4, June 2005. doi: 10.1073/pnas.0503311102. Available online: link. (Cited on page 5.)

[97] Loïc Le Goff and Thomas Lecuit. Developmental biology. Phase transition in a cell. Science (New York, N.Y.), 324(5935):1654-5, June 2009. doi: 10.1126/ science.1176523. Available online: link. (Cited on page 141.)

[98] M Lewis, G Chang, N C Horton, M a Kercher, H C Pace, M a Schumacher, R G Brennan, and P Lu. Crystal structure of the lactose operon repressor and its complexes with DNA and inducer. Science (New York, N.Y.), 271(5253): 1247-54, March 1996. Available online: link. (Cited on page 4.)

[99] Gene-Wei Li and X Sunney Xie. Central dogma at the single-molecule level in living cells. Nature, 475(7356):308-15, July 2011. doi: 10.1038/nature10315. Available online: link. (Cited on pages 3, 6, and 10.)

[100] Jun Li, Liang Wang, Yoshifumi Hashimoto, Chen-Yu Tsao, Thomas K Wood, James J Valdes, Evanghelos Zafiriou, and William E Bentley. A stochastic model of Escherichia coli AI-2 quorum signal circuit reveals alternative synthesis pathways. Molecular systems biology, 2:67, 2006. doi: 10.1038/ msb4100107. (Cited on page 51.)

[101] Francis Lim and S.Peabody David. Mutations that increase the affinity of a translational repressor for RNA. Nucleic Acids Research, 22(18):3748-3752, September 1994. doi: 10.1093/nar/22.18.3748. Available online: link. (Cited on page 5.) 
[102] Daniel Lingwood and Kai Simons. Lipid rafts as a membrane-organizing principle. Science (New York, N.Y.), 327(5961):46-50, January 2010. doi: 10. 1126/science.1174621. Available online: link. (Cited on page 140.)

[103] Andrew C Liu, David K Welsh, Caroline H Ko, Hien G Tran, Eric E Zhang, Aaron a Priest, Ethan D Buhr, Oded Singer, Kirsten Meeker, Inder M Verma, Francis J Doyle, Joseph S Takahashi, and Steve a Kay. Intercellular coupling confers robustness against mutations in the SCN circadian clock network. Cell, 129(3):605-16, May 2007. doi: 10.1016/j.cell.2007.02.047. Available online: link. (Cited on page 32.)

[104] Chenli Liu, Xiongfei Fu, Lizhong Liu, Xiaojing Ren, Carlos K L Chau, Sihong Li, Lu Xiang, Hualing Zeng, Guanhua Chen, Lei-Han Tang, Peter Lenz, Xiaodong Cui, Wei Huang, Terence Hwa, and Jian-Dong Huang. Sequential establishment of stripe patterns in an expanding cell population. Science (New York, N.Y.), 334(6053):238-41, October 2011. doi: 10.1126/science.1209042. Available online: link. (Cited on pages 27, 28, and 140.)

[105] Adiel Loinger, Azi Lipshtat, Nathalie Balaban, and Ofer Biham. Stochastic simulations of genetic switch systems. Physical Review E, 75(2):1-14, February 2007. doi: 10.1103/PhysRevE.75.021904. Available online: link. (Cited on page 143.)

[106] Tao Long, Kimberly C Tu, Yufang Wang, Pankaj Mehta, N P Ong, Bonnie L Bassler, and Ned S Wingreen. Quantifying the integration of quorum-sensing signals with single-cell resolution. PLoS biology, 7(3):e68, 2009. doi: 10.1371/ journal.pbio.1000068. Available online: link. (Cited on page 104.)

[107] T. Lu, D. Volfson, L. Tsimring, and J. Hasty. Cellular growth and division in the Gillespie algorithm. Systems Biology, 1(1):121, 2004. doi: 10.1049/sb: 20045016. Available online: link. (Cited on page 78.)

[108] Ting Lu, Tongye Shen, Matthew R Bennett, Peter G Wolynes, and Jeff Hasty. Phenotypic variability of growing cellular populations. Proceedings of the National Academy of Sciences of the United States of America, 104(48):18982-7, November 2007. doi: 10.1073/pnas.0706115104. (Cited on page 181.)

[109] Noreen L Lyell, Anne K Dunn, Jeffrey L Bose, and Eric V Stabb. Bright mutants of Vibrio fischeri ES114 reveal conditions and regulators that control bioluminescence and expression of the lux operon. Journal of bacteriology, 192 (19):5103-14, October 2010. doi: 10.1128/JB.00524-10. Available online: link. (Cited on pages 68 and 104.)

[110] Hédia Maamar, Arjun Raj, and David Dubnau. Noise in gene expression determines cell fate in Bacillus subtilis. Science (New York, N.Y.), 317(5837): 526-9, July 2007. doi: 10.1126/science.1140818. Available online: link. (Cited on page 19.) 
[111] H H McAdams and a Arkin. Stochastic mechanisms in gene expression. Proceedings of the National Academy of Sciences of the United States of America, 94 (3):814-9, February 1997. Available online: link. (Cited on page 7.)

[112] Margaret McFall-Ngai, Elizabeth a C Heath-Heckman, Amani a Gillette, Suzanne M Peyer, and Elizabeth a Harvie. The secret languages of coevolved symbioses: insights from the Euprymna scolopes-Vibrio fischeri symbiosis. Seminars in immunology, 24(1):3-8, February 2012. doi: 10.1016/j.smim.2011. 11.006. Available online: link. (Cited on page 25.)

[113] Kathleen E McGinness, Tania a Baker, and Robert T Sauer. Engineering controllable protein degradation. Molecular cell, 22(5):701-7, June 2006. doi: 10.1016/j.molcel.2006.04.027. Available online: link. (Cited on page 124.)

[114] D. McMillen, N. Kopell, J. Hasty, and JJ Collins. Synchronizing genetic relaxation oscillators by intercell signaling. Proceedings of the National Academy of Sciences of the United States of America, 99(2):679, 2002. (Cited on pages 31 and 43.$)$

[115] Pontus Melke, Patrik Sahlin, Andre Levchenko, and Henrik Jönsson. A cellbased model for quorum sensing in heterogeneous bacterial colonies. PLoS computational biology, 6(6):e1000819, January 2010. doi: 10.1371/journal.pcbi. 1000819. Available online: link. (Cited on page 69.)

[116] Petros Mina, Mario di Bernardo, Nigel J Savery, and Krasimira TsanevaAtanasova. Modelling emergence of oscillations in communicating bacteria: a structured approach from one to many cells. Journal of the Royal Society, Interface / the Royal Society, 10(78):20120612, January 2013. doi: 10.1098/rsif. 2012.0612. Available online: link. (Cited on pages 64, 69, and 190.)

[117] M. I. More, L. D. Finger, J. L. Stryker, C. Fuqua, A. Eberhard, and S. C. Winans. Enzymatic Synthesis of a Quorum-Sensing Autoinducer Through Use of Defined Substrates. Science, 272(5268):1655-1658, June 1996. doi: 10.1126/science.272.5268.1655. (Cited on page 40.)

[118] Marco J Morelli, Rosalind J Allen, Sorin Tanase-Nicola, and Pieter Rein ten Wolde. Eliminating fast reactions in stochastic simulations of biochemical networks: a bistable genetic switch. The Journal of chemical physics, 128(4):045105, 2008. doi: 10.1063/1.2821957. Available online: link. (Cited on page 135.)

[119] K.H. KH Nealson, Terry Platt, and JW Hastings. Cellular control of the synthesis and activity of the bacterial luminescent system. Journal of bacteriology, 104(1):313, 1970. Available online: link. (Cited on pages 20 and 67.)

[120] Nuno R Nené, Jordi Garca-Ojalvo, and Alexey Zaikin. Speed-dependent cellular decision making in nonequilibrium genetic circuits. PLoS One, 7(3): 
e32779, January 2012. doi: 10.1371/journal.pone.0032779. Available online: link. (Cited on page 106.)

[121] Wai-Leung Ng and Bonnie L Bassler. Bacterial quorum-sensing network architectures. Annual review of genetics, 43:197-222, 2009. doi: 10.1146/ annurev-genet-102108-134304. (Cited on pages 21, 22, 25, 26, 38, and 68.)

[122] Spencer V Nyholm and Margaret J McFall-Ngai. The winnowing: establishing the squid-vibrio symbiosis. Nature reviews. Microbiology, 2(8):632-42, August 2004. doi: 10.1038/nrmicro957. Available online: link. (Cited on pages 21 and 22.)

[123] E.M. Ozbudak, M. Thattai, H.N. Lim, B.I. Shraiman, and A. Van Oudenaarden. Multistability in the lactose utilization network of Escherichia coli. $\mathrm{Na}$ ture, 427(6976):737-740, 2004. Available online: link. (Cited on pages 4, 13, $16,104,109,118$, and 204.)

[124] Ertugrul M Ozbudak, Mukund Thattai, Iren Kurtser, Alan D Grossman, and Alexander van Oudenaarden. Regulation of noise in the expression of a single gene. Nature genetics, 31(1):69-73, 2002. doi: 10.1038/ng869. (Cited on pages 10 and 79.)

[125] Anand Pai and Lingchong You. Optimal tuning of bacterial sensing potential. Molecular systems biology, 5(286):286, 2009. doi: 10.1038/msb.2009.43. (Cited on pages $31,47,48$, and 69 .)

[126] Anand Pai, Yu Tanouchi, Cynthia H Collins, and Lingchong You. Engineering multicellular systems by cell-cell communication. Current opinion in biotechnology, 20(4):461-70, 2009. doi: 10.1016/j.copbio.2009.08.006. Available online: link. (Cited on pages 27, 179, and 204.)

[127] M R Parsek, D L Val, B L Hanzelka, J E Cronan, and E P Greenberg. Acyl homoserine-lactone quorum-sensing signal generation. Proceedings of the $\mathrm{Na}$ tional Academy of Sciences of the United States of America, 96(8):4360-5, April 1999. (Cited on page 40.)

[128] J Paulsson and M Ehrenberg. Random signal fluctuations can reduce random fluctuations in regulated components of chemical regulatory networks. Physical review letters, 84(23):5447-50, June 2000. Available online: link. (Cited on page 13.)

[129] Johan Paulsson. Summing up the noise in gene networks. Nature, 427(6973): 415-8, January 2004. doi: 10.1038/natureo2257. Available online: link. (Cited on page 13.) 
[130] JP Pearson and KM Gray. Structure of the autoinducer required for expression of Pseudomonas aeruginosa virulence genes. Proceedings of the ..., 91 (January):197-201, 1994. Available online: link. (Cited on page 22.)

[131] J.P. Pearson, C. Van Delden, and B.H. Iglewski. Active efflux and diffusion are involved in transport of Pseudomonas aeruginosa cell-to-cell signals. Journal of bacteriology, 181(4):1203, 1999. (Cited on page 51.)

[132] J. Peccoud and B. Ycart. Markovian modeling of gene-product synthesis. Theoretical Population Biology, 48(2):222-234, 1995. (Cited on page 5.)

[133] Juan M Pedraza and Johan Paulsson. Effects of molecular memory and bursting on fluctuations in gene expression. Science (New York, N.Y.), 319(5861): 339-43, 2009. doi: 10.1126/science.1144331. Available online: link. (Cited on page 136.$)$

[134] Juan M Pedraza and Alexander van Oudenaarden. Noise propagation in gene networks. Science (New York, N.Y.), 307(5717):1965-9, March 2005. doi: 10.1126/science.1109090. Available online: link. (Cited on pages 14 and 15.)

[135] Pablo D Pérez, Joel T Weiss, and Stephen J Hagen. Noise and crosstalk in two quorum-sensing inputs of Vibrio fischeri. BMC systems biology, 5:153, January 2011. doi: 10.1186/1752-0509-5-153. Available online: link. (Cited on page 103.)

[136] Pablo Delfino Pérez and Stephen J Hagen. Heterogeneous response to a quorum-sensing signal in the luminescence of individual Vibrio fischeri. PLoS One, 5(11):e15473, January 2010. doi: 10.1371/journal.pone.0015473. Available online: link. (Cited on pages 34, 67, 103, and 205.)

[137] Brian M Peters, Mary Ann Jabra-Rizk, Graeme a O’May, J William Costerton, and Mark E Shirtliff. Polymicrobial interactions: impact on pathogenesis and human disease. Clinical microbiology reviews, 25(1):193-213, January 2012. doi: 10.1128/CMR.00013-11. Available online: link. (Cited on page 24.)

[138] Benjamin Pfeuty and Kunihiko Kaneko. The combination of positive and negative feedback loops confers exquisite flexibility to biochemical switches. Physical biology, 6(4):046013, January 2009. doi: 10.1088/1478-3975/6/4/ 046013. Available online: link. (Cited on page 104.)

[139] A. Pikovsky, A. Zaikin, and M. de La Casa. System Size Resonance in Coupled Noisy Systems and in the Ising Model. Physical Review Letters, 88(5):2-5, 2002. doi: 10.1103/PhysRevLett.88.050601. Available online: link. (Cited on page 181.) 
[140] Arthur Prindle, Phillip Samayoa, Ivan Razinkov, Tal Danino, Lev S Tsimring, and Jeff Hasty. A sensing array of radically coupled genetic 'biopixels'. Nature, 481(7379):39-44, January 2012. doi: 10.1038/nature10722. Available online: link. (Cited on pages 27 and 179.)

[141] Priscilla E M Purnick and Ron Weiss. The second wave of synthetic biology: from modules to systems. Nature reviews. Molecular cell biology, 10(6):410-22, June 2009. doi: 10.1038/nrm2698. Available online: link. (Cited on pages 109, 137, and 204.)

[142] Nan Qin, Sean M Callahan, Paul V Dunlap, and Ann M Stevens. Analysis of LuxR regulon gene expression during quorum sensing in Vibrio fischeri. Journal of bacteriology, 189(11):4127-34, 2007. doi: 10.1128/JB.01779-06. Available online: link. (Cited on page 26.)

[143] Arjun Raj and Alexander van Oudenaarden. Nature, nurture, or chance: stochastic gene expression and its consequences. Cell, 135(2):216-26, 2008. doi: 10.1016/j.cell.2008.09.050. (Cited on pages 12, 14, and 203.)

[144] Arjun Raj and Alexander van Oudenaarden. Single-molecule approaches to stochastic gene expression. Annual review of biophysics, 38:255-70, 2009. doi: 10.1146/annurev.biophys.37.032807.125928. (Cited on page 3.)

[145] Arjun Raj, Scott a Rifkin, Erik Andersen, and Alexander van Oudenaarden. Variability in gene expression underlies incomplete penetrance. Nature, 463 (7283):913-8, February 2010. doi: 10.1038/natureo8781. Available online: link. (Cited on page 15.)

[146] L Ravn, AB Christensen, S Molin, M Givskov, and L. Gram. Methods for detecting acylated homoserine lactones produced by Gram-negative bacteria and their application in studies of AHL-production kinetics. Journal of Microbiological Methods, 44(3):239-251, 2001. (Cited on page 38.)

[147] G Reshes, S Vanounou, I Fishov, and M Feingold. Timing the start of division in E. coli: a single-cell study. Physical biology, 5(4):046001, January 2008. doi: 10.1088/1478-3975/5/4/046001. Available online: link. (Cited on page 83.)

[148] Rudolf Rigler. Fluorescence correlations, single molecule detection and large number screening Applications in biotechnology. Journal of Biotechnology, 41 (2-3):177-186, July 1995. doi: 10.1016/0168-1656(95)ooo54-T. Available online: link. (Cited on page 5.)

[149] Lydia Robert, Gregory Paul, Yong Chen, François Taddei, Damien Baigl, and Ariel B Lindner. Pre-dispositions and epigenetic inheritance in the Escherichia coli lactose operon bistable switch. Molecular systems biology, 6(357): 357, April 2010. doi: 10.1038/msb.2010.12. Available online: link. (Cited on page 106.) 
[150] Corbette Roberts, Kelsi L Anderson, Ellen Murphy, Steven J Projan, William Mounts, Barry Hurlburt, Mark Smeltzer, Ross Overbeek, Terrence Disz, and Paul M Dunman. Characterizing the effect of the Staphylococcus aureus virulence factor regulator, SarA, on log-phase mRNA half-lives. Journal of bacteriology, 188(7):2593-603, 2006. doi: 10.1128/JB.188.7.2593-2603.2006. (Cited on pages 49 and 83.)

[151] Katherine W Rogers and Alexander F Schier. Morphogen gradients: from generation to interpretation. Annual review of cell and developmental biology, 27: 377-407, January 2011. doi: 10.1146/annurev-cellbio-092910-154148. Available online: link. (Cited on page 139.)

[152] Francisco J Romero-Campero and Mario J Pérez-Jiménez. A model of the quorum sensing system in Vibrio fischeri using P systems. Artificial Life, 14 (1):95-109, January 2008. doi: 10.1162/artl.2008.14.1.95. Available online: link. (Cited on page 69.)

[153] Nitzan Rosenfeld, Jonathan W Young, Uri Alon, Peter S Swain, and Michael B Elowitz. Gene regulation at the single-cell level. Science (New York, N.Y.), 307 (5717):1962-5, 2005. doi: 10.1126/science.1106914. (Cited on pages 11, 63, 77, 83 , and 116 .)

[154] E G Ruby and M.J. McFall-Ngai. A Squid That Glows in the Night : Development of an Animal- Bacterial Mutualism. Journal of bacteriology, 174(15): 4865-4870, 1992. (Cited on page 21.)

[155] Edward G Ruby and Lee Kyu-Ho. The Vibrio fischeri-Euprymna scolopes Light Organ Association : Current Ecological Paradigms. Applied and environmental microbiology, 64(3):805-812, 1998. (Cited on page 21.)

[156] Robert P Ryan and J Maxwell Dow. Communication with a growing family: diffusible signal factor (DSF) signaling in bacteria. Trends in microbiology, 19 (3):145-52, March 2011. doi: 10.1016/j.tim.2010.12.003. Available online: link. (Cited on page 24.)

[157] Yasushi Saka, Cédric Lhoussaine, Celine Kuttler, Ekkehard Ullner, and Marco Thiel. Theoretical basis of the community effect in development. BMC systems biology, 5(1):54, January 2011. doi: 10.1186/1752-0509-5-54. Available online: link. (Cited on pages 20, 139, and 204.)

[158] Alvaro Sanchez, Sandeep Choubey, and Jane Kondev. Regulation of noise in gene expression. Annual review of biophysics, 42:469-91, January 2013. doi: 10.1146/annurev-biophys-083012-130401. Available online: link. (Cited on page 6.) 
[159] Michael a Savageau. Alternative designs for a genetic switch: analysis of switching times using the piecewise power-law representation. Mathematical biosciences, 180:237-53, 2002. Available online: link. (Cited on page 105.)

[16o] AL Schaefer, DL Val, BL Hanzelka, JE Cronan, and EP Greenberg. Generation of cell-to-cell signals in quorum sensing: acyl homoserine lactone synthase activity of a purified Vibrio fischeri LuxI protein. Proceedings of the National Academy of Sciences, 93(18):9505, 1996. (Cited on page 26.)

[161] Martin Schuster, D Joseph Sexton, Stephen P Diggle, and E Peter Greenberg. Acyl-homoserine lactone quorum sensing: from evolution to application. Annual review of microbiology, 67:43-63, September 2013. doi: 10.1146/annurev-micro-092412-155635. Available online: link. (Cited on pages 24 and 26.)

[162] Matthew Scott, Terence Hwa, and Brian Ingalls. Deterministic characterization of stochastic genetic circuits. Proceedings of the National Academy of Sciences of the United States of America, 104(18):7402-7, May 2007. doi: 10.1073/pnas.0610468104. Available online: link. (Cited on pages 35, 108, 110, and 137.)

[163] Alecia N Septer and Eric V Stabb. Coordination of the arc regulatory system and pheromone-mediated positive feedback in controlling the Vibrio fischeri lux operon. PloS one, 7(11):e4959o, January 2012. doi: 10.1371/journal.pone. o04959o. Available online: link. (Cited on pages 68 and 104.)

[164] GS Shadel and TO Baldwin. Identification of a distantly located regulatory element in the luxD gene required for negative autoregulation of the Vibrio fischeri luxR gene. Journal of Biological Chemistry, 267(11):7690, 1992. Available online: link. (Cited on page 69.)

[165] V Shahrezaei, J.F. Ollivier, and P.S. Swain. Colored extrinsic fluctuations and stochastic gene expression. Molecular systems biology, 4(1):196, 2008. (Cited on page 63.)

[166] Vahid Shahrezaei and Peter S Swain. Analytical distributions for stochastic gene expression. Proceedings of the National Academy of Sciences of the United States of America, 105(45):17256-61, November 2008. doi: 10.1073/pnas. 0803850105. (Cited on pages 10, 40, and 61.)

[167] Rafael Silva-Rocha and Víctor de Lorenzo. Noise and robustness in prokaryotic regulatory networks. Annual review of microbiology, 64:257-75, January 2010. doi: 10.1146/annurev.micro.091208.073229. Available online: link. (Cited on pages 13 and 15.) 
[168] Kai Simons and Mathias J Gerl. Revitalizing membrane rafts: new tools and insights. Nature reviews. Molecular cell biology, 11(10):688-99, October 2010. doi: 10.1038/nrm2977. Available online: link. (Cited on page 140.)

[169] Michael L Simpson, Chris D Cox, and Gary S Sayler. Frequency domain analysis of noise in autoregulated gene circuits. Proceedings of the National Academy of Sciences of the United States of America, 100(8):4551-6, April 2003. doi: 10.1073/pnas.0736140100. Available online: link. (Cited on page 13.)

[170] D.M. Sitnikov, G.S. Shadel, and T.O. Baldwin. Autoinducer-independent mutants of the LuxR transcriptional activator exhibit differential effects on the twolux promoters ofVibrio fischeri. Molecular E general genetics : MGG, 252 (5):622-625, 1996. Available online: link. (Cited on pages 68 and 104.)

[171] Carl Song, Hilary Phenix, Vida Abedi, Matthew Scott, Brian P Ingalls, Mads Kaern, and Theodore J Perkins. Estimating the stochastic bifurcation structure of cellular networks. PLoS computational biology, 6(3):e10oo699, March 2010. doi: 10.1371/journal.pcbi.1000699. Available online: link. (Cited on page 110.)

[172] Valeria Specchia, Lucia Piacentini, Patrizia Tritto, Laura Fanti, Rosalba D'Alessandro, Gioacchino Palumbo, Sergio Pimpinelli, and Maria P Bozzetti. Hsp9o prevents phenotypic variation by suppressing the mutagenic activity of transposons. Nature, 463(7281):662-5, February 2010. doi: 10.1038/ natureo8739. Available online: link. (Cited on page 15.)

[173] Ann M Stevens, Martin Schuster, and Kendra P Rumbaugh. Working together for the common good: cell-cell communication in bacteria. Journal of bacteriology, 194(9):2131-41, May 2012. doi: 10.1128/JB.00143-12. Available online: link. (Cited on page 24.)

[174] Paul D Straight and Roberto Kolter. Interspecies chemical communication in bacterial development. Annual review of microbiology, 63:99-118, January 2009. doi: 10.1146/annurev.micro.091208.073248. Available online: link. (Cited on pages 24 and 25.)

[175] Gürol M Süel, Jordi Garcia-Ojalvo, Louisa M Liberman, and Michael B Elowitz. An excitable gene regulatory circuit induces transient cellular differentiation. Nature, 440(7083):545-50, March 2006. doi: 10.1038/nature04588. Available online: link. (Cited on pages 13, 18, and 19.)

[176] Gürol M Süel, Rajan P Kulkarni, Jonathan Dworkin, Jordi Garcia-Ojalvo, and Michael B Elowitz. Tunability and noise dependence in differentiation dynamics. Science (New York, N.Y.), 315(5819):1716-9, March 2007. doi: 10.1126/science.1137455. Available online: link. (Cited on pages 18 and 19.) 
[177] P.S. Swain, M.B. Elowitz, and E.D. Siggia. Intrinsic and extrinsic contributions to stochasticity in gene expression. Proceedings of the National Academy of Sciences, 99(20):12795, 2002. (Cited on pages 11 and 61.)

[178] Yuichi Taniguchi, Paul J Choi, Gene-Wei Li, Huiyi Chen, Mohan Babu, Jeremy Hearn, Andrew Emili, and X Sunney Xie. Quantifying E. coli proteome and transcriptome with single-molecule sensitivity in single cells. Science (New York, N.Y.), 329(5991):533-8, July 2010. doi: 10.1126/science.1188308. Available online: link. (Cited on pages 7 and 61.)

[179] Yu Tanouchi, Dennis Tu, Jungsang Kim, and Lingchong You. Noise reduction by diffusional dissipation in a minimal quorum sensing motif. PLoS computational biology, 4(8):e1000167, 2008. doi: 10.1371/journal.pcbi.1000167. (Cited on pages $37,39,49,65,69$, and 180.)

[180] Yosuke Tashiro, Yutaka Yawata, Masanori Toyofuku, Hiroo Uchiyama, and Nobuhiko Nomura. Interspecies Interaction between Pseudomonas aeruginosa and Other Microorganisms. Microbes and Environments, 28(1):13-24, 2013. doi: 10.1264/jsme2.ME12167. Available online: link. (Cited on page 24.)

[181] Shu-Wen Teng, Yufang Wang, Kimberly C Tu, Tao Long, Pankaj Mehta, Ned S Wingreen, Bonnie L Bassler, and N P Ong. Measurement of the copy number of the master quorum-sensing regulator of a bacterial cell. Biophysical journal, 98(9):2024-31, May 2010. doi: 10.1016/j.bpj.2010.01.031. Available online: link. (Cited on pages 30, 34, 68, 70, 79, 83, 103, and 104.)

[182] M Thattai and a van Oudenaarden. Intrinsic noise in gene regulatory networks. Proceedings of the National Academy of Sciences of the United States of America, 98(15):8614-9, July 2001. doi: 10.1073/pnas.151588598. Available online: link. (Cited on page 13.)

[183] Mukund Thattai. Stochastic Gene Expression in Fluctuating Environments. Genetics, 167(1):523-530, May 2004. doi: 10.1534/genetics.167.1.523. Available online: link. (Cited on page 17.)

[184] Tianhai Tian and Kevin Burrage. Stochastic models for regulatory networks of the genetic toggle switch. Proceedings of the National Academy of Sciences of the United States of America, 103(22):8372-7, May 2006. doi: 10.1073/pnas. 0507818103. Available online: link. (Cited on pages 68 and 143.)

[185] Abhinav Tiwari, J Christian J Ray, Jatin Narula, and Oleg a Igoshin. Bistable responses in bacterial genetic networks: designs and dynamical consequences. Mathematical biosciences, 231(1):76-89, May 2011. doi: 10.1016/j.mbs. 2011.03.004. Available online: link. (Cited on page 105.) 
[186] Alexander Tomasz. Control of the Competent State in Pneumococcus by a Hormone-Like Cell Product: An Example for a New Type of Regulatory Mechanism in Bacteria. Nature, 208(5006):155-159, October 1965. Available online: link. (Cited on page 20.)

[187] FJ Trueba and LJH Koppes. Exponential growth of Escherichia coli B/r during its division cycle is demonstrated by the size distribution in liquid culture. Archives of microbiology, 169:491-496, 1998. Available online: link. (Cited on pages 83 and 135.)

[188] L S Tsimring, D Volfson, and J Hasty. Stochastically driven genetic circuits. Chaos (Woodbury, N.Y.), 16(2):026103, 2006. doi: 10.1063/1.2209571. (Cited on page 43.)

[189] Kimberly C Tu, Tao Long, Sine L Svenningsen, Ned S Wingreen, and Bonnie L Bassler. Negative feedback loops involving small regulatory RNAs precisely control the Vibrio harveyi quorum-sensing response. Molecular cell, 37(4):56779, February 2010. doi: 10.1016/j.molcel.2010.01.022. Available online: link. (Cited on pages 34,70 , and 103.)

[19o] ML Urbanowski, CP Lostroh, and EP Greenberg. Reversible acyl-homoserine lactone binding to purified Vibrio fischeri LuxR protein. Journal of bacteriology, 186(3):631, 2004. doi: 10.1128/JB.186.3.631.2004. Available online: link. (Cited on page 82. )

[191] Nicolaas Godfried Van Kampen. Stochastic processes in physics and chemistry. North holland, 1992. (Cited on page 112.)

[192] Sarah L Veatch, Pietro Cicuta, Prabuddha Sengupta, Aurelia HonerkampSmith, David Holowka, and Barbara Baird. Critical fluctuations in plasma membrane vesicles. ACS chemical biology, 3(5):287-93, May 2008. doi: 10. 1021/cb800012x. Available online: link. (Cited on page 141.)

[193] Karen L Visick and Edward G Ruby. Vibrio fischeri and its host: it takes two to tango. Current opinion in microbiology, 9(6):632-8, December 2006. doi: 10.1016/j.mib.2006.10.001. Available online: link. (Cited on pages 23 and 25.)

[194] Junwei Wang, Jiajun Zhang, Zhanjiang Yuan, and Tianshou Zhou. Noiseinduced switches in network systems of the genetic toggle switch. BMC systems biology, 1:50, January 2007. doi: 10.1186/1752-0509-1-50. Available online: link. (Cited on pages 68, 137, 143, and 177.)

[195] L. Wang, Y. Hashimoto, C.Y. Tsao, J.J. Valdes, and W.E. Bentley. Cyclic AMP (cAMP) and cAMP receptor protein influence both synthesis and uptake of extracellular autoinducer 2 in Escherichia coli. Journal of bacteriology, 187(6): 2066, 2005. doi: 10.1128/JB.187.6.2066. (Cited on page 51.) 
[196] Patrick B Warren and Pieter Rein ten Wolde. Chemical models of genetic toggle switches. The journal of physical chemistry. B, 109(14):6812-23, 2005. doi: 10.1021/jpo45523y. Available online: link. (Cited on pages 143 and 145.)

[197] Marc Weber and Javier Buceta. Dynamics of the quorum sensing switch: stochastic and non-stationary effects. BMC systems biology, 7(1):6, January 2013. doi: 10.1186/1752-0509-7-6. Available online: link. (Cited on page 110.)

[198] Joshua W Williams, Xiaohui Cui, Andre Levchenko, and Ann M Stevens. Robust and sensitive control of a quorum-sensing circuit by two interlocked feedback loops. Molecular systems biology, 4(234):234, 2008. doi: 10.1038/msb. 2008.70. Available online: link. (Cited on pages 21, 26, 69, 73, 79, 80, 82, 83, $86,87,89,94,104$, and 106.)

[199] Paul Williams and Miguel Cámara. Quorum sensing and environmental adaptation in Pseudomonas aeruginosa: a tale of regulatory networks and multifunctional signal molecules. Current opinion in microbiology, 12(2):18291, April 2009. doi: 10.1016/j.mib.2009.01.005. Available online: link. (Cited on page 174.)

[20o] K.B. Xavier and B.L. Bassler. Regulation of uptake and processing of the quorum-sensing autoinducer AI-2 in Escherichia coli. Journal of bacteriology, 187(1):238, 2005. doi: 10.1128/JB.187.1.238. (Cited on page 51.)

[201] Lingchong You, Robert Sidney Cox, Ron Weiss, and Frances H Arnold. Programmed population control by cell-cell communication and regulated killing. Nature, 428(6985):868-71, 2004. doi: 10.1038/nature02491. (Cited on page 27.)

[202] Ji Yu, Jie Xiao, Xiaojia Ren, Kaiqin Lao, and X Sunney Xie. Probing gene expression in live cells, one protein molecule at a time. Science (New York, N.Y.), 311(5767):1600-3, 2006. doi: 10.1126/science.1119623. (Cited on pages 6, 38,52 , and 79 .)

[203] Anna Zakharova, Jürgen Kurths, Tatyana Vadivasova, and Aneta Koseska. Analysing dynamical behavior of cellular networks via stochastic bifurcations. PloS one, 6(5):e19696, January 2011. doi: 10.1371/journal.pone.0019696. Available online: link. (Cited on pages 35, 110, and 137.)

[204] Xiu-Deng Zheng, Xiao-Qian Yang, and Yi Tao. Bistability, probability transition rate and first-passage time in an autoactivating positive-feedback loop. PloS one, 6(3):e17104, January 2011. doi: 10.1371/journal.pone.0017104. Available online: link. (Cited on pages 106 and 110.) 
[205] Tianshou Zhou, Luonan Chen, and Kazuyuki Aihara. Molecular Communication through Stochastic Synchronization Induced by Extracellular Fluctuations. Physical Review Letters, 95(17):2-5, 2005. doi: 10.1103/PhysRevLett.95. 178103. (Cited on page 102.)

[206] Jun Zhu, Melissa B Miller, Russell E Vance, Michelle Dziejman, Bonnie L Bassler, and John J Mekalanos. Quorum-sensing regulators control virulence gene expression in Vibrio cholerae. Proceedings of the National Academy of Sciences of the United States of America, 99(5):3129-34, March 2002. doi: 10. 1073/pnas.052694299. Available online: link. (Cited on page 22.)

[207] Pawel J Zuk, Marek Kochanczyk, Joanna Jaruszewicz, Witold Bednorz, and Tomasz Lipniacki. Dynamics of a stochastic spatially extended system predicted by comparing deterministic and stochastic attractors of the corresponding birth-death process. Physical biology, 9(5):055002, October 2012. doi: 10.1088/1478-3975/9/5/055002. Available online: link. (Cited on page 116.) 



\section{COLOPHON}

This document was typeset using the typographical look-and-feel classicthesis developed by André Miede. The style was inspired by Robert Bringhurst's seminal book on typography "The Elements of Typographic Style". classicthesis is available for both LTEX and LYX:

http://code.google.com/p/classicthesis/

Final Version as of April 1, 2014 (classicthesis version 10.0). 